\title{
Microwave Ferrites, Part 1: Fundamental properties
}

\author{
Ümit Özgürí, Yahya Alivov, and Hadis Morkoç \\ Department of Electrical and Computer Engineering \\ Virginia Commonwealth University \\ Richmond, VA 23284 \\ Received: 5 January 2009
}

\begin{abstract}
Ferrimagnets having low RF loss are used in passive microwave components such as isolators, circulators, phase shifters, and miniature antennas operating in a wide range of frequencies $(1-100 \mathrm{GHz})$ and as magnetic recording media owing to their novel physical properties. Frequency tuning of these components has so far been obtained by external magnetic fields provided by a permanent magnet or by passing current through coils. However, for high frequency operation the permanent part of magnetic bias should be as high as possible, which requires large permanent magnets resulting in relatively large size and high cost microwave passive components. A promising approach to circumvent this problem is to use hexaferrites, such as $\mathrm{BaFe}_{12} \mathrm{O}_{19}$ and $\mathrm{SrFe}_{12} \mathrm{O}_{19}$, which have high effective internal magnetic anisotropy that also contributes to the permanent bias. Such a self-biased material remains magnetized even after removing the external applied magnetic field, and thus, may not even require an external permanent
\end{abstract}

\footnotetext{
i E-mail: uozgur@vcu.edu
} 
Özgür et al., submitted to Journal of Materials Science: Materials in Electronics, 2009 Microwave Ferrites, Part 1: Fundamental properties

magnet. In garnet and spinel ferrites, such as $\mathrm{Y}_{3} \mathrm{Fe}_{5} \mathrm{O}_{12}$ (YIG) and $\mathrm{MgFe}_{2} \mathrm{O}_{4}$, however, the uniaxial anisotropy is much smaller, and one would need to apply huge magnetic fields to achieve such high frequencies. In Part 1 of this review of microwave ferrites a brief discussion of fundamentals of magnetism, particularly ferrimagnetism, and chemical, structural, and magnetic properties of ferrites of interest as they pertain to net magnetization, especially to self biasing, are presented. Operational principles of microwave passive components and electrical tuning of magnetization using magnetoelectric coupling are discussed in Part 2. 
Özgür et al., submitted to Journal of Materials Science: Materials in Electronics, 2009

Microwave Ferrites, Part 1: Fundamental properties

\section{TABLE OF CONTENTS}

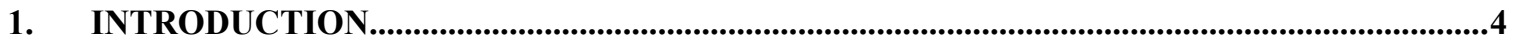

2. A PRIMER TO MAGNETISM AND MAGNETIC MATERIALS ...............................................11

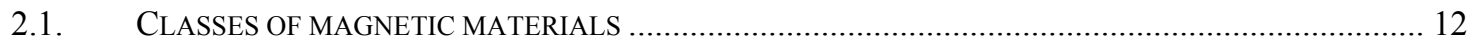

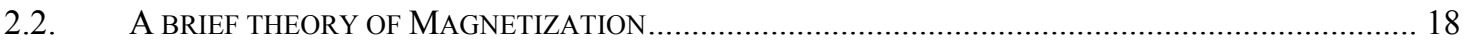

3. PHYSICAL STRUCTURE LEADING TO FERROELECTRICITY, FERROMAGNETISM,

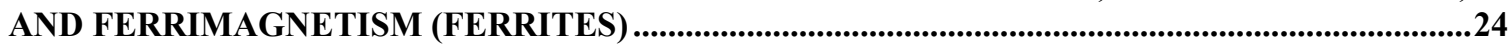

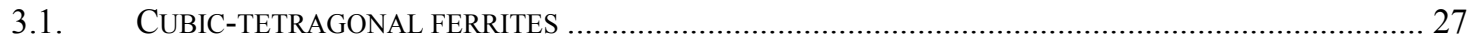

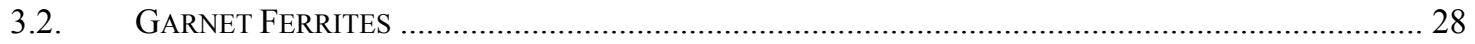

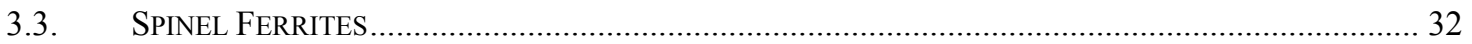

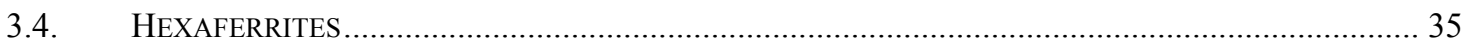

4. A PRIMER TO THE THEORY OF FERRIMAGNETISM .....................................................39

4.1. FERROMAGNETIC RESONANCE AND THE PERMEABILITY TENSOR ................................................. 41

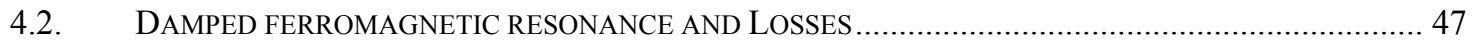

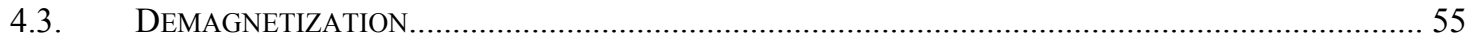

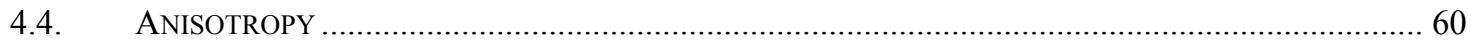

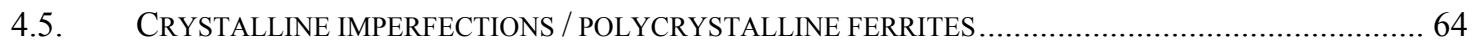

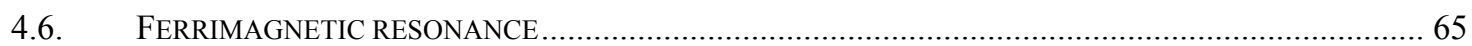

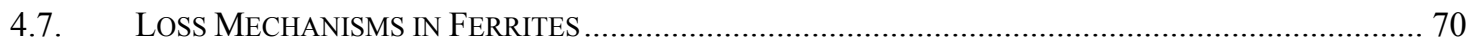

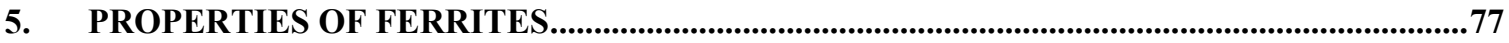

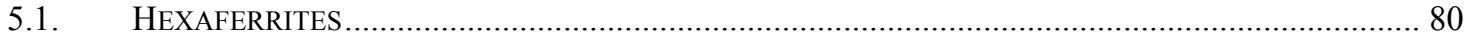

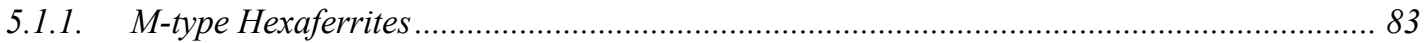

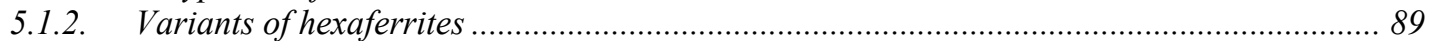

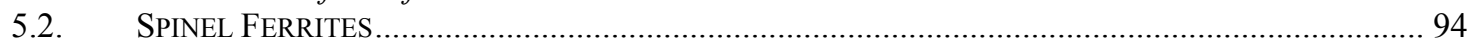

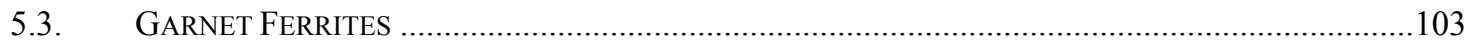

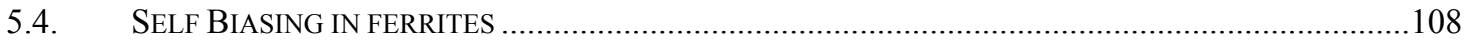

6. SUMMARY AND OUTLOOK ...............................................................................................110

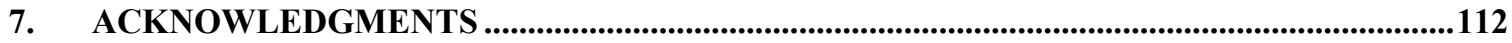


Özgür et al., submitted to Journal of Materials Science: Materials in Electronics, 2009 Microwave Ferrites, Part 1: Fundamental properties

\section{Introduction}

Oxides and semiconductors containing transition metal elements and some rare earths as dopants or constituents exhibit ferromagnetic and ferrimagnetic (antiferromagnetic) behavior if the spin of the magnetic ions are aligned. In addition processes such as interaction with the host material can spin polarize the host's electrons in the same direction increasing the Bohr magneton beyond that which can be expected from the magnetic ion by itself. Oxides with transition metal (or rare earth) as a constituent are of special interest as they exhibit large permeability whose magnitude is dependent in many cases on the variations in the structural properties (atomic arrangement) which are affected by factors such as strain which can be externally applied. Among the subclass of oxides using $\mathrm{Fe}$ as a constituent and showing antiferromagnetism is a class of materials which go by the name of ferrimagnets. High resistivity (low loss) varieties of ferrimagnets find a plethora of applications in passive microwave components such as isolators, circulators, phase shifters, and miniature antennas operating at a wide range of frequencies (1-100 GHz) and as magnetic recording media using their novel physical properties to cite a few. ${ }^{1}$

Magnets have become an integral part of human endeavor, and are found in many industries such as automobile, telecommunication, data processing, electronics, and instrumentation. For example, in automotive industry they are used in starter motors, anti-lock braking systems (ABS), motor drives for wipers, injection pumps, fans and controls for windows, seats etc., loudspeakers, eddy current brakes, and alternators. In telecommunications, they find applications in speakers, microphones, telephone ringers, 
Özgür et al., submitted to Journal of Materials Science: Materials in Electronics, 2009 Microwave Ferrites, Part 1: Fundamental properties

electro-acoustic pick-ups, switches and relays, core components for many microwave passive components including miniaturized strip antennas. In data processing, we find magnets in disc drives and actuators, stepping motors, and printers. In consumer electronics, they prominently showcase themselves as DC motors for showers, washing machines, drills and like tools, low voltage DC drives for cordless appliances, loudspeakers for TV and audio, TV beam correction and focusing device, compact-disc drives, home computers, video recorders, and clocks. In terms of the electronics and instrumentation, they find applications as sensors, contactless switches, Nuclear Magnetic Resonance (NMR) spectrometer, energy meter disc, electro-mechanical transducers, crossed field tubes, flux-transfer shunt trip device, and dampers. They find wide ranging application in industry as DC motors for magnetic tools, robotics, magnetic separators for extracting metals and ores, magnetic bearings, servo-motor drives, lifting apparatus, brakes and clutches, meters and measuring equipment. In astro and aerospace, frictionless bearings, stepping motors, couplings, instrumentation, traveling wave tubes, and autocompass utilize magnets. Last but not least, magnets and or magnetic materials can be found in dentures, orthodontics, orthopedics, wound closures, stomach seals, repulsion collars, ferromagnetic probes, cancer cell separators, magnetomotive artificial hearts, NMR / Magnetic Resonance Imaging (MRI), and targeted drug delivery by magnetic nanoparticles.

The development of magnetism, as in any field, has been a fascinating one. The history of permanent magnetism is very rich and unlike many aspects of science dates back tens of centuries. The earliest observations of magnetism can be traced back to the Greek 
Özgür et al., submitted to Journal of Materials Science: Materials in Electronics, 2009 Microwave Ferrites, Part 1: Fundamental properties

philosopher Thales in the $6^{\text {th }}$ Century B.C. However, it was not until 1600 that the understanding of magnetism began, which paved the way for the modern treatment of magnetism. Early on what was termed to be magnetite (a naturally occurring oxide $\mathrm{Fe}_{3} \mathrm{O}_{4}$ ), contained in naturally occurring rocks, was found to attract one another. These rocks were named after the province Magnesia in Asia Minor (today's Turkey) and all materials exhibiting mutual attraction were given the same name thereafter. This is true also for the first metallic magnet material introduced by William Gilbert in 1600 in the form of an iron compass needle. Gilbert referred to this special type of material as hardened iron and this is perhaps the very reason that even today one still speaks of magnetic hardness although there is no direct correlation to the mechanical hardness. ${ }^{2}$ Given below, is a timeline chronicling the very notable turning points in the development of magnetism. ${ }^{\text {ii }}$

1600: William Gilbert published the first systematic experiments on magnetism in "De Magnete".

1819: Oersted serendipitously made the connection between magnetism and electricity discovering that a current carrying wire deflected a compass needle.

1825: Sturgeon discovered the electromagnet.

1880: Warburg obtained the first hysteresis loop for iron.

1895: The Curie law was developed.

1905: Langevin explained the theory of diamagnetism and paramagnetism.

1906: Weiss proposed theory for ferromagnetism.

1920's: The physics of magnetism got developed which included theories based on electron spins and exchange interactions.

The progress in magnetism in general has been mind-boggling as exemplified by the digital and other recording media. The density of recording and the information that can

\footnotetext{
ii http://www.magnets.bham.ac.uk/magnetic_materials/history.htm
} 
Özgür et al., submitted to Journal of Materials Science: Materials in Electronics, 2009 Microwave Ferrites, Part 1: Fundamental properties

be packed in and read in ever smaller dimensions has been responsible for the progress along with modern processing technologies which are often shared with Si technology and has been increasing by leaps and bounds for decades. The progress in permanent magnets has paralleled in many ways the aforementioned development. A case in point is that permanent magnets reduced the size of motors considerably and allow stepping motors to be developed with very high rotational accuracy which form the back bone of hard drives in computers. In fact one of those permanent magnets, barium hexaferrite $\left(\mathrm{BaFe}_{12} \mathrm{O}_{19}\right)$, has become so pervasive that about $50 \mathrm{~g}$ of it is produced per year for every person on earth. This particular magnetic material is also ideal for microwave passive components which will be discussed in more detail in this review.

The progress in magnetic materials has been breathtaking in that each time a magnetic material was developed it was supplanted by a new one. This trend is shown in Figure 1 which depicts the development of magnets throughout the $20^{\text {th }}$ century. The figure tracks the figure of merit, maximum energy product which is the product of magnetic induction and maximum magnetic field (in terms of $\mathrm{kJm}^{-3}$ and also GaussOe), over the years and shows doubling of this figure every 12 years.

Let us now give a very brief description of milestones in the field of modern magnets and magnetism. The first recognized permanent magnetic material was lodestone (naturally occurring $\mathrm{Fe}_{3} \mathrm{O}_{4}$ oxide - magnetite). It is postulated that large magnetic field generated by lightning strikes were responsible for magnetizing the rocks. It is the resistance to demagnetization that is the salient feature of the rocks involved. The next notable 
Özgür et al., submitted to Journal of Materials Science: Materials in Electronics, 2009 Microwave Ferrites, Part 1: Fundamental properties

development came in the form of the magnetic carbon steel developed in the early $18^{\text {th }}$ century. When steel is alloyed with $\mathrm{W}$ and/or $\mathrm{Cr}$ to form carbide precipitates under requisite heat treatment, domain wall motion is obstructed. These magnets are superior to lodestone in terms of magnetic saturation but inferior in terms of demagnetization. 1930s ushered in the development of alnico magnets, based on, $\mathrm{Ni}, \mathrm{Co}$, and $\mathrm{Fe}$ with small amounts of $\mathrm{Al}, \mathrm{Cu}$ and $\mathrm{Ti}$ (typical weight $\%$ : Fe-35, Co-35, Ni-15, Al-7, Cu-4, Ti-4), which are considered to be the first modern permanent magnets that considerably improved magnetic hardness (resistance to demagnetization) over magnetic steel. Alnico magnets contain ferromagnetic Fe-Co needles in a non-magnetic Al-Ni matrix. Although they are not as hard as some of the magnetic materials that followed, the high Curie temperature, approximately $1120 \mathrm{~K}$, make them still attractive.

Boosted by the war effort (WWII), hard magnets such as $\left(\mathrm{BaFe}_{12} \mathrm{O}_{19}\right.$ or $\left.\mathrm{SrFe}_{12} \mathrm{O}_{19}\right)$ experienced considerable development. Sr ferrite has slightly superior magnetic properties though. Owing to their large crystallographic anisotropy, they exhibit large coercivity and substantial resistance to demagnetization, but the energy product $\left(\mathrm{BH}_{\max }\right)$ is low. Low production cost together with the aforementioned properties pave the way for wide spread use, e.g. electric motors and everyday magnets. Of paramount interest to this review is the fact that large anisotropic field and low Eddy current loss make these magnetic materials ideally suited for microwave applications. As such this class of magnetic materials is covered in great detail in this review. The 1960s and the era that followed saw substantial development in magnetic materials containing rare earths, such as samarium cobalt $\left(\mathrm{SmCo}_{5}\right)$ and yttrium cobalt $\left(\mathrm{YCo}_{5}\right)$ both of which make use of the 
Özgür et al., submitted to Journal of Materials Science: Materials in Electronics, 2009 Microwave Ferrites, Part 1: Fundamental properties

phase based on a rare-earth (RE) and a transition metal (TM). The combination of RE and $\mathrm{TM}$ is ideal as the RE element provides the anisotropy to the phase and the TM material leads to high magnetization and Curie temperature. This development was soon followed by the discovery of $\mathrm{SmCo}_{5}$ in 1967 , becoming the first commercial RE/TM permanent magnetic material. In fact $\mathrm{SmCo}_{5}$ held the record for the highest energy product for many years and is stable at high temperatures, albeit at the expense of high cost.

Moreover, neodymium iron boron magnets, developed in 1980s, sport a good combination of high saturation magnetization and good resistance to demagnetization, but they suffer from relatively low Curie temperature $(585 \mathrm{~K})$ and high cost. Addition of Co and Dy improves the temperature characteristics but at the expense of increased cost. Despite the high cost, these magnets find applications in cases where small size, miniaturization, is a coveted property. Permanent magnets such as samarium iron nitride offer high resistance to demagnetization, high magnetization, and importantly increased resistance to corrosion and high temperature compared with neodymium iron boron magnets.

Fast forwarding, a variety of soft ferrites was soon developed. Soft magnetic material is more of a technical term than a scientific one. The word "soft" means temporary in the sense that the ferromagnetism emerges only when a magnetic field is applied. In contrast, hard magnets display ferromagnetism in the absence of an external field. All magnetic elements in the pure form are soft, whereas magnetic solid solutions and compounds can be either soft or hard. However, the metallic soft magnetic materials cannot be used at 
Özgür et al., submitted to Journal of Materials Science: Materials in Electronics, 2009 Microwave Ferrites, Part 1: Fundamental properties

high frequencies due to Eddy current losses. For this purpose, ceramic insulator based soft ferrites became the desirable material. As previously discussed, these materials are ferrimagnetic with a cubic crystal structure and the general composition of the type $\mathrm{MeO} \cdot \mathrm{Fe}_{2} \mathrm{O}_{3}$, where Me specifies a transition metal such as nickel, manganese, or zinc. $\mathrm{MnZn}$ ferrite can be used at frequencies up to $10 \mathrm{MHz}$, for example in telephone signal transmitters and receivers and in switch mode power supplies (also referred to as DC-DC converters). Additionally, part of the family of soft ferrites, are the microwave ferrites, e.g. yttrium iron garnet (YIG) to be discussed in this review. These ferrites are used in the frequency range from $100 \mathrm{MHz}$ to $500 \mathrm{GHz}$, for waveguides for electromagnetic radiation and in microwave devices such as phase shifters, isolators, and circulators to name a few.

This review paper is organized as follows: Following a succinct discussion of fundamentals of the origin of magnetism and magnetic and in particular ferromagnetic materials, structural properties of various types of magnetic materials as they pertain to net magnetization are discussed. This is followed by the theory of ferrimagnetism with emphasis on ferromagnetic resonance, permeability tensor, anisotropy, etc. This then paves the way to the discussion of three classes of ferrites, namely hexaferrites, spinel ferrites, and garnet ferrites. Part 2 of this review ${ }^{3}$ discusses operational principles of microwave passive magnetic components in addition to applications such as phase shifters, circulators, isolators, filters, and ferrite antennas, and the fundamentals of magnetoelectric (ME) coupling and its use for tuning magnetic properties with electric field. 


\section{A primer to magnetism and magnetic materials}

Because this review deals with magnetic properties of mainly the ferrites, it is incumbent upon us to give a succinct review of magnetization and various classes of magnetic materials. The topic is discussed in many books and the reader is recommended to refer to them for a more expansive discussion. ${ }^{4}$

Transition metals and rare earths with incomplete $3 d$ and $4 f$ shells, respectively, are an integral part of magnetism in general and ferromagnetic and ferrimagnetic materials in particular. Sometimes deviation from stoichiometry is also used to cause spin polarization without the need for transition metal being present. This, however, is beyond the scope of this review and therefore will not be discussed. Transition-metal elements have valence electrons corresponding to the $4 s$ orbital but have partially filled $3 d$ shells, thus the name $3 d$ transition metals (i.e. Mn with the shell structure of $1 s^{2} 2 s^{2} 2 p^{6} 3 s^{2} 3 p^{6} 3 d^{5} 4 s^{2}$ ). In a free or isolated transition metal ion where all the electronic shells but the $3 d$ shell is filled, the ionic ground state would be degenerate if the electrons were not to interact with each other. However, this degeneracy, albeit not completely, is lifted by electron-electron Coulomb interaction and electron spin orbit interaction. The lowest levels after the degeneracy is lifted are governed by a simple set of rules: Russel-Saunders coupling (or LS coupling), and Hund's rules, which come about as a result of complex calculations. ${ }^{5}$ According to these rules, the $3 d$ band of the $\mathrm{Mn}^{2+}$ ion is exactly half-filled with 5

electrons among the 10 available states, with a gap between the up-spin $(\uparrow)$ occupied 
Özgür et al., submitted to Journal of Materials Science: Materials in Electronics, 2009 Microwave Ferrites, Part 1: Fundamental properties

states and empty down-spin $(\downarrow)$ states. For other transition metals, such as Fe, Co, Ni, one of the bands is usually partially filled (up or down), as shown in Table 1.

For an element with $53 d$ electrons $(\mathrm{Mn})$ this means that all 5 electrons would have spins up. The total spin, $\mathrm{S}$, is calculated as $\mathrm{S}=(1 / 2)(\mathrm{n} \downarrow-\mathrm{n} \uparrow)$ which for $\mathrm{Mn}(3 d)$ would give $(1 / 2)(5)=5 / 2$ because all $d$ shell electrons have the same spin. In Fe, however, there are 6 $3 d$ electrons and one of them would have to have a spin down configuration. The $4 s$ state has two electrons with opposite spins. In this case the total spin would be $(1 / 2)(5-1)=2$. The methodology to how the electrons are arranged in the $d$ shell for transition metals including orbital angular momentum, spin angular momentum, total momentum and the name designation for each of the available $10 d$ shell states are shown in Table 1.

\subsection{Classes of magnetic materials}

The genesis of magnetism has to do with the orbital and spin motions of electrons and how the electrons interact with one another as pertained to their spin. The classification of magnetic materials is based on how they respond to magnetic fields. Although as surprising as it may sound, all matter is magnetic to varying degrees. The main delineating factor is that in some materials there is no collective long range interaction between atomic magnetic moments, whereas in other materials there is a very strong interaction. The magnetic behavior of materials can be classified into the following five major groups: diamagnetic, paramagnetic, ferromagnetic, antiferromagnetic, and 
Özgür et al., submitted to Journal of Materials Science: Materials in Electronics, 2009 Microwave Ferrites, Part 1: Fundamental properties

ferrimagnetic. Alternatively, in terms of the phenomena, they are: diamagnetism, paramagnetism, ferromagnetism, antiferromagnetism, and ferrimagnetism.

Although it is usually very weak, diamagnetism is a fundamental property of all matter. Diamagnetic substances are composed of atoms which have no net magnetic moments (i.e., all the orbital shells are filled and there are no unpaired electrons). However, when exposed to a magnetic field, a negative magnetization is produced against the applied magnetic field and thus the susceptibility, $\chi$, is negative and ranges from $-5.0 \times 10^{-9}$ for Silicon to $-1.6 \times 10^{-4}$ for Bismuth. As shown in Table 2, magnetization $M$ changes linearly with applied magnetic field $H$ with a negative slope (susceptibility is both negative and small). Another well known characteristic of diamagnetic materials is that the susceptibility is temperature independent. Diamagnetism arises from the tendency of electrical charge partially screening the interior of the body from the applied magnetic field and negative susceptibility can be interpreted as material being poised to expel the applied field. All non magnetic materials are diamagnetic including all semiconductors such as $\mathrm{Si}, \mathrm{Ge}, \mathrm{GaAs}, \mathrm{GaN}$, etc. except when doped with some transition metal elements such as Mn or rare earths such as Gd to render them magnetic.

Electronic paramagnetism arises from e.g. atoms, molecules, and lattice defects, possessing an odd number of electrons (unpaired) causing a nonzero total spin. Free atoms and ions with a partially filled inner shell such as transition elements, ions isoelectronic with transition elements, rare earth, and actinide elements have unpaired electrons in those shells which lead to non vanishing spin. Examples include $\mathrm{V}^{2+}, \mathrm{Cr}^{2+}$, 
Özgür et al., submitted to Journal of Materials Science: Materials in Electronics, 2009 Microwave Ferrites, Part 1: Fundamental properties

$\mathrm{Mn}^{2+}, \mathrm{Fe}^{2+}, \mathrm{Co}^{2+}, \mathrm{Ni}^{2+}$ among the transition elements and $\mathrm{Gd}^{3+}$ among the rare earths. The magnetization $M$ vs. magnetic field $H$ curve in these materials follows a linear relationship with a positive slope and positive magnetization, $\chi>0$, unlike the diamagnetic materials, as shown in Table 2. Among several theories concerning paramagnetism, the Langevin model, applicable to materials with non-interacting localized electrons, states that each atom has a magnetic moment which is randomly oriented as a result of thermal agitations. Application of a magnetic field creates a slight alignment of these moments and thus a low magnetization in the same direction as the applied field. As the temperature increases, however, retention of alignment becomes harder due to the thermal agitations and thus the susceptibility decreases, as described by the Curie Law, which will be discussed in Section 2.2 (see Equation 6). In the Pauli model of paramagnetism the conduction electrons are considered essentially to be free and under an applied field an imbalance between electrons with opposite spin is set up leading to a low magnetization in the same direction as the applied field. The susceptibility is independent of temperature, although the electronic band structure may be affected, which will then have an effect on the magnitude of the susceptibility.

Ferromagnetism is only possible when atoms are arranged in a lattice and the atomic magnetic moments can interact to align parallel to each other, resulting in a positive and large susceptibility and a non-zero magnetic moment even at zero applied field, called the spontaneous magnetic moment. Ferromagnetism was initially described by a classical theory which assumed the presence of a molecular field within the ferromagnetic material, 
Özgür et al., submitted to Journal of Materials Science: Materials in Electronics, 2009 Microwave Ferrites, Part 1: Fundamental properties

postulated first by Weiss in 1907. In quantum mechanics, however, the Heisenberg model of ferromagnetism is used, describing the parallel alignment of magnetic moments in terms of an exchange interaction between neighboring moments. Among the elements only $\mathrm{Fe}, \mathrm{Co}$ and $\mathrm{Ni}$ are ferromagnetic at and above room temperature. Below a critical temperature called the Curie temperature the magnetization $M$ vs. $H$ curve for a ferromagnet shows hysteresis. Above Curie temperature, the thermal agitation becomes so great that the material turn into paramagnetic. The Curie temperatures for the abovementioned naturally ferromagnetic elements are $T_{C}(\mathrm{Fe})=1043 \mathrm{~K}, T_{C}(\mathrm{Co})=1404 \mathrm{~K}$ and $T_{C}(\mathrm{Ni})=631 \mathrm{~K}$.

In the strict definition of ferromagnetism, a material is ferromagnetic only if all of its magnetic ions are aligned and add a positive contribution to the net magnetization. If some of the magnetic ions subtract from the net magnetization (if they are partially antialigned), the material exhibits ferrimagnetism. In the extreme case where the sublattices with anti-aligned magnetic moments result in zero net magnetization, despite the magnetic ordering, the material exhibits antiferromagnetism. All of these anti-alignment effects only take place at temperatures below the Curie temperature, $T_{C}$, (for ferromagnets and ferrimagnets) or the Néel temperature, $T_{N}$, (for antiferromagnets). Antiferromagnetic materials typically become paramagnetic above the Néel temperature. The only element exhibiting antiferromagnetism at room temperature is chromium with a Néel temperature of $37{ }^{\circ} \mathrm{C}$. The clue to antiferromagnetism is the behavior of susceptibility above the Néel temperature where the susceptibility obeys the Curie-Weiss 
Özgür et al., submitted to Journal of Materials Science: Materials in Electronics, 2009 Microwave Ferrites, Part 1: Fundamental properties

law for paramagnets but with a negative intercept indicating negative exchange interactions. Antiferromagnetic materials possess small but positive susceptibility.

Ferrimagnetic materials such as ferrites which utilize transition element $\mathrm{Fe}$ as in $\mathrm{Fe}_{2} \mathrm{O}_{3}$ exhibit magnetism similar to ferromagnetic materials below the Curie temperature, $T_{C}$. Above this temperature, they become paramagnetic as in the case of ferromagnetic materials. Ferrimagnetism is observed only in compounds with complex crystal structures. In these materials the exchange interactions lead to parallel alignment of atoms in some of the crystal sites, sublattice A, and anti-parallel alignment of others, those in sublattice B. The material breaks down into magnetic domains, as in ferromagnetic materials. ${ }^{6}$ The main difference between ferrimagnetic and antiferromagnetic materials is that the magnetic moment associated with sublattice A atoms is larger than, as opposed to being equal to, that of sublattice B atoms so they do not null each other. The magnetic behavior in ferrimagnetic and ferromagnetic materials is also very similar. However, ferrimagnetic materials generally have lower saturation magnetizations. Ultimately, the magnitude of the spontaneous magnetization depends on how well the sublattice moments are aligned, as thermal vibration of the atoms causes misalignment of the moments and a reduction in magnetization. For ferrimagnetic materials not all of the moments align parallel, even at zero Kelvin, and hence spontaneous magnetization will depend on the relative alignment of the moments as well as the temperature. 
Özgür et al., submitted to Journal of Materials Science: Materials in Electronics, 2009 Microwave Ferrites, Part 1: Fundamental properties

As an example, in Barium ferrite $\left(\mathrm{BaO} \cdot 6 \mathrm{Fe}_{2} \mathrm{O}_{3}\right)$ the unit cell contains 64 ions of which the barium and oxygen ions have no magnetic moment, $16 \mathrm{Fe}^{3+}$ ions have moments aligned parallel and $8 \mathrm{Fe}^{3+}$ ions aligned anti-parallel giving a net magnetization parallel to the applied field. The amplitude of the net magnetization, however, is relatively low as only $1 / 8$ of the ions contribute to the magnetization of the material. Because ferromagnetic materials are typically non-conducting and consequently do not suffer from eddy current and associated loss they are useful in radio frequency applications. In this review we will consider ferrites as microwave ferrites due to their applications in microwave and also in millimeter wave systems as critical elements in passive components.

Having learned various types of magnetization, we now present in Table 3 magnetization type, saturation magnetization at room temperature (298 K), and Néel temperature for some magnetic materials and ferrites.

Because anisotropy is an integral part of magnetization, a few words on the topic is warranted. Magnetic properties vary depending on the crystallographic direction along which the magnetic dipoles are aligned. For example, magnetite (naturally occurring $\mathrm{Fe}_{3} \mathrm{O}_{4}$ ), which has a spinel structure, can be magnetized along the $<111>$ like directions, the easy direction of magnetization. The $<100>$ like directions represent the hard direction of magnetization and the $<110>$ like directions are the intermediate direction of magnetization, as shown in Figure 2(a). Furthermore, as an example to magnetic materials with hexagonal crystalline structure, Co can be magnetized along the [0001] 
Özgür et al., submitted to Journal of Materials Science: Materials in Electronics, 2009 Microwave Ferrites, Part 1: Fundamental properties

direction but has a hard direction along the $<10 \overline{1} 0>$ like directions which lie in the basal plane, as shown in Figure 2(b). A measure of the magnetocrystalline anisotropy in the easy direction is the anisotropy field, $H_{a}$, as shown in Figure 2, which is the field needed for rotating all the magnetic moments by $90^{\circ}$ as one unit when the applied field reaches the saturation field. Coupling of the electron orbitals to the lattice causes this anisotropy. In the easy direction, this coupling is such that the electron orbitals are their lowest energy state. The easy direction of a permanent magnet based on ferrite or rare earth alloys must be uniaxial, although it is possible in some materials to have more than one easy axis or where the easy direction can lie on a certain plane or the surface of a cone. Permanent magnets have uniaxial symmetry and it is difficult to demagnetize them as they are resistant to rotation of the direction of magnetization.

\subsection{A brief theory of Magnetization}

In diamagnetic and paramagnetic materials, small applied magnetic fields lead to an internal magnetic induction which can be expressed as (with the assumption that demagnetization effects are negligible and the internal magnetic field $\mathbf{H}_{\text {int }}$ can be approximated by the external magnetic field $\mathbf{H}$ which is justified for diamagnetic and paramagnetic materials)

$$
\mathbf{B}_{i n t}=\mathbf{B}+\mu_{0} \mathbf{M}=\mu_{0}(\mathbf{H}+\mathbf{M})
$$

\section{Equation 1}

where $\mu_{0}$ represents the permeability of free space, $\mathbf{B}$ is the external magnetic induction, $\mathbf{H}$ is the external magnetic field, and $\mathbf{M}$ is the magnetization. In the case of 
Özgür et al., submitted to Journal of Materials Science: Materials in Electronics, 2009 Microwave Ferrites, Part 1: Fundamental properties

ferromagnetic materials, however, the internal field $\mathbf{H}_{\text {int }}$ cannot be approximated by the external $\mathbf{H}$ field, and the relationship in Equation 1 is true for the external $\mathbf{B}$ field instead:

$$
\mathbf{B}=\mathbf{H}+4 \pi \mathbf{M} \text { in cgs or } \mathbf{B}=\mu_{0}(\mathbf{H}+\mathbf{M}) \text { in SI units }
$$

\section{Equation 2}

Magnetization, $\mathbf{M}$, is defined as the magnetic moment per unit volume. The magnetic polarization, $J_{M}$, also called the intensity of magnetization, is defined as $J_{M}=\mu_{0} M$ and its saturation value is depicted with the nomenclature of $J_{s}$. Similarly, the saturation values of magnetization is depicted by $M_{S}$. Magnetic susceptibility, $\chi$, is the ratio of magnetization divided by the macroscopic $\mathbf{H}$ field:

$$
\chi=\mathbf{M} / \mathbf{H}
$$

\section{Equation 3}

The magnitude of susceptibility serves to classify different materials as tabulated in Table 2. As discussed in Section 2.1 dealing with classes of magnetic materials, the materials can be classified as diamagnetic with negative and small magnetic susceptibility, paramagnetic with negative and large or positive and small magnetic susceptibility, ferromagnetic with positive but small magnetic susceptibility, antiferromagnetic with positive but small magnetic susceptibility, and ferromagnetic with positive and large magnetic susceptibility. Because cgs and SI units are used in the literature, conversion factors between the two as well as definition of pertinent magnetism parameters along with their units are tabulated in Table 4. 
Özgür et al., submitted to Journal of Materials Science: Materials in Electronics, 2009 Microwave Ferrites, Part 1: Fundamental properties

It is instructive to briefly discuss the rudimentary basis for magnetism in an effort to get acquainted with the terminology, basis for various types of magnetism, temperature dependence of magnetism, and values of fundamental nature such as Bohr magneton. In this realm let us consider $N$ atoms per unit volume each with a magnetic moment $\mathbf{m}$. Magnetic field, if any present, will align those moments, but thermal disorder would resist the tendency to align. The energy of interaction of the moment $\mathbf{m}$ with the applied field $\mathbf{B}$ is given by: ${ }^{4}$

$$
U=-\mathbf{m} \cdot \mathbf{B}
$$

\section{Equation 4}

In thermal equilibrium, the magnetization is given by the Langevin equation as

$$
M=N m L(x)
$$

\section{Equation 5}

where $x \equiv \frac{m B}{k T}$ and the Langevin function $L(x)=\operatorname{ctnh} x-1 / x$. For $x<<1$ (or $m B<<k T) L(x) \approx x / 3$, which leads to the well known Curie law:

$$
M \approx \frac{N m^{2} B}{3 k T}=\frac{C}{T} \frac{B}{\mu_{0}}=\chi H
$$

\section{Equation 6}

where $k$ is the Boltzmann's constant and $C$ is the Curie constant and is given by $C \equiv N m^{2} / 3 k$. It should be noted that in some cases the Curie law is expressed in cgs units, with $H$ replaced by $B$, since the applied field $B$ can be expressed as $\mu_{0} H$, and $\mu_{0}=1$ in cgs units. The Langevin function for this regime is then expressed as $L(x) \cong x / 3=m B / 3 k T$. Magnetic moment of an atom or ion in free space is given by 
Özgür et al., submitted to Journal of Materials Science: Materials in Electronics, 2009 Microwave Ferrites, Part 1: Fundamental properties

$$
\mathbf{m}=\gamma \hbar \mathbf{J}=-g \mu_{B} \mathbf{J}
$$

\section{Equation 7}

where $\hbar \mathbf{J}$ is the total angular momentum for the electronic system of an atom, which is the sum of the orbital angular momentum $\hbar \mathbf{L}$ and spin angular momentum $\hbar \mathbf{S}, \gamma$ is the gyromagnetic or magnetogyric ratio $\left(\gamma=g e / 2 m_{e}=g \mu_{B} / \hbar\right), \mu_{B}$ is the Bohr magneton ( $e \hbar / 2 m_{e} c$ in cgs and $e \hbar / 2 m_{e}$ in SI units, where $e$ is the electronic charge, $m_{e}$ is the electron mass, and $\hbar$ is reduced Plank's constant), and $g$ is called the Landé $g$-factor, spectroscopic splitting factor, or Landé splitting factor as the magnitude of this factor determines how rapidly the energy levels split. For an electron the orbital g-factor is equal to $g_{L}=1$ and the spin g-factor is equal to $g_{S} \approx 2.0023$ which is often truncated to 2 . For the ground state $(L=0, J=1 / 2)$ the Landé g-factor is $g=g_{S} \approx 2$ as given by the Landé equation, which results in $\gamma=1.759 \times 10^{11} \mathrm{C} / \mathrm{kg}$ for the free electron.

In the realm of basics of magnetization, for atoms in a magnetic field one should consider quantized and equally spaced energy levels $(2 J+1$ in total) defined by the angular momentum quantum number $J$. The magnetization in this case is expressed as

$$
M=N g J \mu_{B} B_{J}(x) \text { where } x \equiv \frac{g J \mu_{B} B}{k T}
$$

\section{Equation 8}

and $B_{J}(x)$ is the Brillouin function given by

$$
B_{J}(x)=\frac{2 J+1}{2 J} \operatorname{ctnh}\left[\frac{(2 J+1)}{2 J} x\right]-\frac{1}{2 J} \operatorname{ctnh}\left(\frac{x}{2 J}\right)
$$

\section{Equation 9}

which is equal to the Langevin function in the limit $J \rightarrow \infty$. For $\mathrm{x}<<1$ one obtains 
Özgür et al., submitted to Journal of Materials Science: Materials in Electronics, 2009 Microwave Ferrites, Part 1: Fundamental properties

$$
\frac{M}{B / \mu_{0}}=\chi \approx \frac{N J(J+1) g^{2} \mu_{B}^{2}}{3 k T}=\frac{N p^{2} \mu_{B}^{2}}{3 k T}=\frac{C}{T}
$$

\section{Equation 10}

where $p \equiv g \sqrt{J(J+1)}$ is the effective number of Bohr magnetons. If we set $J=1 / 2$

(ground state) the electron magnetization is obtained as

$$
M=\frac{N \mu_{B}^{2}}{k T} B
$$

Equation 11

which is temperature dependent. In the Pauli model of paramagnetism the Fermi Dirac statistics are applied to magnetization resulting in a total magnetization (including both paramagnetic and diamagnetic contribution)

$$
M \approx \frac{N \mu_{B}^{2}}{k T_{F}} B
$$

\section{Equation 12}

which is temperature independent in agreement with observations. Here, $E_{F}=k T_{F}$ is the Fermi energy. In the absence of an external magnetic field, Pauli magnetism at absolute zero also indicates that the number of electrons in the spin "up" and spin "down" states adjusts to make the energies equal at the Fermi level as shown in Figure 3. However, when a magnetic field is applied, electrons with one spin, in this case spin down, are moved in energy, albeit very small compared to that observed in the ferromagnetic state due to the large effective molecular field. Consequently, there would be an excess of spin up electrons that are not spin paired, as shown in Figure 3 (b). In ferromagnetic materials be it magnetic semiconductor or ferromagnet below the critical temperature, the picture depicted in Figure 3 (b) would hold figuratively without the external magnetic field. 
Özgür et al., submitted to Journal of Materials Science: Materials in Electronics, 2009 Microwave Ferrites, Part 1: Fundamental properties

Given that the energy level of the system in magnetic field is $E=m_{J} g \mu_{B} B$, for a simple spin with azimuthal quantum number $m_{J}= \pm 1 / 2$ and without orbital moment the total energy splitting of an electron state is given by $\Delta E=2 \mu_{B} B$ assuming $g=2$ as shown in Figure 3 (b).

To investigate the transition of a system of spins from paramagnetic to ferromagnetic state, many-body problem is reduced to a much simplified effective one-body problem using the Mean Field Theory (MFT), where an exchange interaction field $\mathbf{H}_{E}=\lambda_{E} \mathbf{M}$ (in cgs units) in addition to the applied field $\mathbf{H}$ is introduced. The total susceptibility in terms of the temperature-independent constant $\lambda_{E}$ can be found as

$$
\chi=\frac{C}{T-C \lambda_{E}} \text { or } \chi=\frac{C}{T-\Theta} \quad \text { in cgs units }
$$

\section{Equation 13}

which represents the Curie-Weiss law. At the critical temperature $T=\Theta=C \lambda_{E}$ a singularity occurs and for $T \leq \lambda_{E} C$ we have spontaneous magnetization. In Equation 13 $\Theta$ can either be positive, negative, or zero. The case of $\Theta=0$ corresponds to the situation when the Curie-Weiss law equates to the Curie law depicted in Equation 10. A non zero $\Theta$ implies that there is an interaction between neighboring magnetic moments and the material is only paramagnetic above a certain transition temperature. If $\Theta$ is positive, the material is ferromagnetic below the transition temperature and the value of $\Theta$ corresponds to the transition temperature (Curie temperature, $T_{C}$ ). If $\Theta$ is negative, the material is antiferromagnetic below the transition temperature (Néel temperature, $T_{N}$ ); however, the value of $\Theta$ does not relate to $T_{N}$. We should note that Equation 13 is only 
Özgür et al., submitted to Journal of Materials Science: Materials in Electronics, 2009 Microwave Ferrites, Part 1: Fundamental properties

valid when the material is in a paramagnetic state. Similarly, it is not valid for many metals as the electrons contributing to the magnetic moment are not localized.

In ferromagnetic samples and for temperatures above the Curie temperature the electronic structure is similar to that shown in Figure 3 (a). However below the Curie temperature, the picture is similar that shown in Figure 3 (b) without any external magnetic field.

\section{Physical structure leading to ferroelectricity, ferromagnetism, and ferrimagnetism (ferrites)}

Ferroelectric and ferromagnetic materials represent a class of materials that exhibit spontaneous electric polarization for the former and magnetic polarization for the latter below a temperature called the Curie temperature. The crystalline structure can be triclinic, monoclinic, orthorhombic, tetragonal, hexagonal, and complex cubic such as spinel. There are 21 classes of non-centro-symmetric structures, a feature which is imperative for piezoelectricity, 20 of which exhibit piezoelectricity. Of these 20 classes, 10 are known as crystals having unique polar axis and crystals belonging to these classes, also called polar crystals, possess spontaneous polarization or electric moment per unit volume. A ferroelectric crystal has two or more orientation states in the absence of electric field and can be shifted from one to another of these states by an external electric field. For this reason it is called reversible, meaning not just the amplitude but also the direction of polarization can be changed. Similarly, a ferromagnetic material has two or more orientation states in the absence of magnetic field and can be reoriented from one to another of these states by a magnetic field, i.e. by magnetic poling. As in the ferroelectric 
Özgür et al., submitted to Journal of Materials Science: Materials in Electronics, 2009 Microwave Ferrites, Part 1: Fundamental properties

case, it is called reversible, meaning not just the amplitude but also the direction of magnetic polarization can be changed.

Ferromagnetic materials have as a constituent material, typically a transition metal such as $\mathrm{Mn}, \mathrm{Fe}$, etc., or a rare earth, such as $\mathrm{Gd}$, etc., which forms the basis for magnetic polarization. Somewhat similarly, ferroelectric materials have an ion, such as $\mathrm{Ti}, \mathrm{Ta}, \mathrm{Zr}$, etc., which is the route of cause of electric polarization. Ferroelectric materials can be represented with a well known triangle, shown in Figure $4,^{7}$ each corner of which represents stress, electric field, and temperature. The branch between the stress and electric field represent the piezoelectricity, the branch between the electrical field and temperature represents the electrothermal effect, and the branch between the thermal and mechanical stimuli represents the thermoelastic effect. The detailed descriptions of connective processes that take place are also indicated between the properties such as strain, displacement, and entropy. Although not shown, magnetic and optical properties are also closely imbricated with the aforementioned three properties of the material but are not shown for clarity.

As in the case of ferroelectric materials, the magnetic and electrical properties of ferromagnetic materials are imbricated through electromechanical constants and magnetostriction coefficients. In other words, a distortion in the lattice structure causes not only an electrical polarization but also a change in the magnetic property through a electromagnetic coupling coefficient, $\alpha_{\mathrm{EM}}$, as shown in Figure 5. Having two fundamental properties to be changed by strain for example suffices to ascribe the term 
Özgür et al., submitted to Journal of Materials Science: Materials in Electronics, 2009 Microwave Ferrites, Part 1: Fundamental properties

multiferroic for the material under consideration. However, this coefficient may not be as large as in the case wherein individually selected ferromagnetic and piezoelectric materials are in solid physical contact with each other. It is in this context that growth of a ferromagnetic (or ferrimagnetic) material and piezoelectric material is gaining a lot of interest. The caveat, however, is that structural mismatch between the two classes of materials may be encountered. This places a limit as to the available pairs that can be grown on each other. Specific to microwave applications, the thickness of the ferrimagnetic material needs to be in the tens of microns which may limit the growth of the bulk of the material to liquid phase epitaxy or hydrothermal method, unless of course new designs are discovered which do not require thick films.

The reversible spontaneous polarization in ferroelectric materials and magnetization in ferromagnetic materials, exhibit a hysteresis loop which is observed below a certain temperature called the Curie temperature, $T_{C}$. At temperatures above the Curie point the electric moments in ferroelectric materials and magnetic moment in ferromagnetic materials in the crystals are disorganized rendering the material non-polar and no longer ferroelectric or ferromagnetic depending on the type. A representative hysteresis loop for a ferroelectric material is shown in Figure 6. In the absence of collective polarization (non-poled) the ferroelectric materials behave like normal dielectrics.

Ferromagnetic and ferrimagnetic materials have non-linear initial magnetization curves (see Figure 7), as the changing magnetization with applied field is due to a change in the magnetic domain structure. Similar to ferroelectric materials, these materials also show 
Özgür et al., submitted to Journal of Materials Science: Materials in Electronics, 2009 Microwave Ferrites, Part 1: Fundamental properties

hysteresis in their magnetization-magnetic field curve below the Curie point, $T_{C}$. The magnetization does not return to zero when the applied magnetic field is returned to zero once a large magnetic field had been applied. Figure 7 shows a typical hysteresis loop; the two loops represent the same data, however, the red solid curve is the magnetic polarization (intensity of magnetization $J_{M}=\mu_{0} M=B-\mu_{0} H$ ) and the black dashed curve the induction, both plotted against the applied magnetic field, $H$.

Structural properties of magnetic ion containing materials and the position as well as the charge state of magnetic elements determine the extent of magnetism. Below we will discuss cubic, spinel, garnet, and hexaferrite type of magnetic materials pointing out their visceral properties and how atomic positions affect their magnetism.

\subsection{Cubic-tetragonal ferrites}

Alnicos, which are high Curie temperature $(\sim 1120 \mathrm{~K})$ magnetic alloys primarily composed of $\mathrm{Al}, \mathrm{Ni}, \mathrm{Co}$, and $\mathrm{Fe}$ and small amounts of $\mathrm{Cu}$ and $\mathrm{Ti}$ (Typical weight \%: Fe35, Co-35, Ni-15, Al-7, Cu-4, Ti-4), were developed as early as 1930s (by Mishima ${ }^{8}$ in 1932 to be accurate). Alnico alloys contain finely dispersed, oriented, and elongated particles precipitated by thermal treatment in a field. These magnetic materials boost good temperature stability and resistance to demagnetization from vibration and shock. In these permanent magnetic materials at the state of high coercive force the crystal structure of the precipitate is distorted from a cubic lattice to a tetragonal lattice. The earlier varieties exhibited coercive fields in the range of $500-800$ Oe with saturation 
Özgür et al., submitted to Journal of Materials Science: Materials in Electronics, 2009 Microwave Ferrites, Part 1: Fundamental properties

magnetization in the range of 1,800-1,900 Gauss. ${ }^{9}$ With continual development, maximum energy products vary approximately in the range $1-9 \times 10^{6}$ Gauss-Oe, coercive forces range from 400 to 2,000 Oe, and the residual induction range from 5,000 to 13,000 Gauss. The alnico alloys are hard and brittle. Alnico 1-4 represent the earlier developments (used in motors and coin selectors in vending machines), and 5 represent the improved version which is also the most widely used alnico alloy. Alnico 8 combines high energy product and coercive force. It is used in electron beam focusing in traveling wave tubes, motors, generators and hearing aids. Alnico 9 combines the highest energy product of all the alnico alloys and high coercive force and is used in loudspeakers, motors, generators, measurement instrumentation. Other cubic magnets such as Cunife (Cunife I consists of $60 \% \mathrm{Cu}, 20 \% \mathrm{Ni}$, and $30 \% \mathrm{Fe}$ by weight), Cunico (Cunico I alloy consists of $50 \% \mathrm{Cu}, 21 \% \mathrm{Ni}$, and $29 \% \mathrm{Co}$ ), Realloy (17\% Mo, $12 \% \mathrm{Co}, 71 \% \mathrm{Fe}$ or 20\% Mo, $12 \%$ Co, and $68 \%$ Fe by weight), Vicalloy (30-52\% Fe, 36-62\% Co, and 4-16\% V with later varieties lowering the $\mathrm{V}$ concentration down to $1 \%$ all by weight), Pt-Co (76.7 \% Pt and 23.3\%Co by weight), $\gamma \mathrm{Fe}_{2} \mathrm{O}_{3}$, and $\mathrm{Fe}_{3} \mathrm{O}_{4}$ have also been developed. There are also iron-cobalt and nickel-cobalt varieties. The cubic magnets are generally thought of as being magnetically soft (which precludes their exposure to significant demagnetizing fields) even though they continually saw improvements in that regard. Consequently and also because this class of ferrites, particularly the earlier varieties, exhibit relatively low intrinsic coercivity other magnetic materials such as hexagonal ferrites have been developed.

\subsection{Garnet Ferrites}


Özgür et al., submitted to Journal of Materials Science: Materials in Electronics, 2009 Microwave Ferrites, Part 1: Fundamental properties

The garnets have orthorhombic crystal structure (oxygen polyhedra, surrounding the cations) but with trivalent cations (including rare earth and $\mathrm{Fe}^{3+}$ ) occupying tetrahedral (d), octahedral (a), or dodecahedral - a 12-sided distorted polyhedral - (c) sites. Specifically, the interaction between tetrahedral and octahedral sites is antiparallel, and the net magnetic moment is antiparallel to the rare earth ions on the $c$ sites. The garnet structure is one of the most complicated crystal structures and it is difficult to draw a twodimensional representation that shows clearly all the ions (160) in the unit cell. For simplicity, only an octant of a garnet structure that shows just the cation positions is shown in Figure 8. The garnet structure is composed of a combination of octahedral (trivalent cation surrounded by six oxygen ions), tetrahedral (trivalent cations surrounded by four oxygen ions), and 12-sided polyhedral- dodecahedral- (trivalent cations surrounded by 8 oxygen atoms) sites, the orientations of which are shown in Figure 9. Specifically the chemical formula for garnets is $3 \mathrm{M}_{2} \mathrm{O}_{3} \cdot 5 \mathrm{Fe}_{2} \mathrm{O}_{3}$ [alternatively dividing all by two $\left.-\mathrm{Me}_{3} \mathrm{Fe}_{2}\left(\mathrm{FeO}_{4}\right)_{3}\right]$ where $\mathrm{Me}$ represents the trivalent rare earth such as non magnetic yttrium or a magnetic rare earth such as from lanthanum through ytterbium. The entire structure, however, contains 4 units of $3 \mathrm{Me}_{2} \mathrm{O}_{3} \cdot 5 \mathrm{Fe}_{2} \mathrm{O}_{3}$. If the $\mathrm{Me}$ ion is nonmagnetic as in the case of yttrium which has no $f$ electrons, the $\mathrm{Fe}^{3+}$ cation ions form two nonidentical antiparallel sublattices in up and down states that result in a net non-zero magnetization of material. Since antiferromagnetic superexchange interaction causes 3 spin up and 2 spin down electrons, a net magnetic moment of $5 \mu_{\mathrm{B}}$ per molecule occurs leading to relatively small magnetization per unit volume. The nature of the superexchange interaction depends not only on the type of the magnetic ion, but strongly depends on the bond length and bonding angle. This makes it possible to change the 
Özgür et al., submitted to Journal of Materials Science: Materials in Electronics, 2009 Microwave Ferrites, Part 1: Fundamental properties

strength and type of the superexchange interaction, i.e. the magnetization and the Néel point, by chemical substitutions of different size ions. Incorporation of magnetic rare earth ions causes formation of a third magnetic sublattice which in turn causes the material to have a compensation point below room temperature. This compensation point can be tuned by substituting trivalent $\mathrm{Al}$ or Ga for trivalent $\mathrm{Fe}$. Unlike the $\mathrm{Fe}_{3} \mathrm{O}_{4}$ like ferrites wherein some $\mathrm{Fe}$ atoms are divalent and some trivalent, all rare earth cations in garnets are trivalent. Consequently, electron hopping through the material is prevented, making the material high resistivity which bodes well for high frequency applications.

Delving deeper into the garnet structure, as mentioned above there are actually four units of $3 \mathrm{Me}_{2} \mathrm{O}_{3} \cdot 5 \mathrm{Fe}_{2} \mathrm{O}_{3}$ or eight units of $\mathrm{Me}_{3} \mathrm{Fe}_{2}\left(\mathrm{FeO}_{4}\right)_{3}$ in the unit cell. Sixteen of the forty $\mathrm{Fe}^{3+}$ cations occupy the octahedral sites each at the center of an octahedron, ${ }^{10}$ as shown in Figure 9. These sites represent the magnetic sublattice which is called A sublattice or $a$ sites. The remaining twenty-four $\mathrm{Fe}^{2+}$ ions occupy the $d$ sites at the center of tetrahedron, and these sites form the D magnetic sublattice. All 24 of the Me (stands for metal in general and when specifically referring to rare earth, RE replaces Me) cations are situated on $24 c$ sites, each site being at the center of an 8-cornered 12-sided polyhedron. There are 96 oxygen ions in a unit cell, each on $h$ site, which is at a point where a corner of a tetrahedron, octahedron, and two large polyhedra meet, ${ }^{11}$ as shown in Figure 9. In contrast to the spinel ferrites, which have two types of crystallographic sites as will be described below, the garnet structure has three types of lattice sites available to metallic ions. The magnitudes of the superexchange interactions between pairs of sublattices depend strongly on the angles between the magnetic ions. The strongest interaction 
Özgür et al., submitted to Journal of Materials Science: Materials in Electronics, 2009 Microwave Ferrites, Part 1: Fundamental properties

occurs for an angle near $180^{\circ}$ and the weakest for an angle near $90^{\circ}$. Therefore, one might expect a relatively strong A-D interaction between the two sublattices occupied by $\mathrm{Fe}^{3+}$ ions because the angle $\mathrm{Fe}^{3+}(a)-\mathrm{O}^{2-}-\mathrm{Fe}^{3+}(d)$ is $126.6^{\circ}$. The coupling between $\mathrm{Me}^{3+}$ and $\mathrm{Fe}^{3+}(a)$ is weaker because the angle $\mathrm{Me}^{3+}-\mathrm{O}^{2-}-\mathrm{Fe}^{3+}(a)$ is $100^{\circ}$. The dominance of the A$\mathrm{D}$ interaction is common to all magnetic garnets and this results in almost the same Néel temperature for all garnets (see Table 3).

Rare earth (RE) ferrites are different from others in that the magnetic moment per ion in $\mathrm{RE}$ is determined not only by spin value (some rare earths used do not even have spin unpaired electrons such as yttrium), but also by orbital contribution because the incomplete $4 f$ layer in $\mathrm{RE}$ elements is partially screened from the crystalline field by the $5 s$ and $5 p$ layers of the xenon core. The strongest interaction in RE garnets is still $\mathrm{Fe}^{3+}-\mathrm{O}^{2-}-\mathrm{Fe}^{3+}$ interaction, which has an angle of $126.6^{\circ}$. The next strongest interaction is between $\mathrm{Fe}^{3+}(d)-\mathrm{O}^{2-}-\mathrm{RE}^{3+}(c)$, which has a smaller angle of $122.2^{\circ}$ and a larger distance $\left[\mathrm{O}^{2-}-\mathrm{RE}^{3+}(c)\right]$ of $2.43 \AA$; this interaction aligns the rare-earth $\mathrm{C}$ sublattice antiparallel to the D sublattice (see Figure 9 for a description of various sublattices). The interaction $\mathrm{RE}^{3+}(c)-\mathrm{O}^{2-}-\mathrm{RE}^{3+}(c)$ within $\mathrm{C}$ sublattice is negligible because of its small angle of $104.7^{\circ}$ meaning small overlap with the oxygen $p$-wave function. The net magnetization of the garnet will be the magnetization of the $\mathrm{C}$ sub-lattice minus the net magnetization of the A-D sublattice: $M_{\text {net }}=M(\mathrm{C})-[M(\mathrm{D})-M(\mathrm{~A})]=M(3 \mathrm{RE})-[M(3 \mathrm{Fe})-M(2 \mathrm{Fe})]$. For gadolinium garnet $\mathrm{Gd}_{3} \mathrm{Fe}_{5} \mathrm{O}_{12}$, for example, the net magnetization at absolute zero temperature will be $M_{n e t}=3 \mu_{\mathrm{RE}}-\left(3 \mu_{\mathrm{Fe}}-2 \mu_{\mathrm{Fe}}\right)=3 \mu_{\mathrm{RE}}-\mu_{\mathrm{Fe}}=3 \times 7 \mu_{\mathrm{B}}-5 \mu_{\mathrm{B}}=16 \mu_{\mathrm{B}}$, where 
Özgür et al., submitted to Journal of Materials Science: Materials in Electronics, 2009 Microwave Ferrites, Part 1: Fundamental properties

$\mu_{\mathrm{RE}}$ and $\mu_{\mathrm{Fe}}$ are magnetic moments of $\mathrm{RE}$ and iron elements, which are equal respectively to $7 \mu_{\mathrm{B}}$ and $5 \mu_{\mathrm{B}}$.

The fairly commonly used $\mathrm{Y}^{3+}$ cation consists of inert krypton core with the $4 p$ (note no $f$ electrons) layer fully filled with six electrons; therefore, since there are no unpaired spins, it has no permanent magnetic moment. The largest size ions, as the nonmagnetic $\mathrm{Y}^{3+}$ ions, are surrounded by eight oxygen ions in a distorted dodecahedral environment $(C$ sites), while the five $\mathrm{Fe}^{3+}$ are divided between three tetrahedral $d$ and two octahedral $a$ sites. To reiterate, the superexchange A-D interaction renders the magnetic moments antiparallel, and is relatively strong because the $\mathrm{Fe}^{3+}-\mathrm{O}^{2-}$ distances are short, and the $\mathrm{Fe}^{3+}-\mathrm{O}^{2-}-\mathrm{Fe}^{3+}$ angle is large enough $\left(126.6^{\circ}\right)$ to permit a substantial overlap of the wave functions of oxygen and ion. So, the net magnetization of YIG arises from three $\mathrm{Fe}^{3+\uparrow}$ ions at $d$ sites minus two $\mathrm{Fe}^{3+}$ at the $a$ sites, giving rise to ferrimagnetism: $3 \mathrm{Fe}^{3+\uparrow}-$ $2 \mathrm{Fe}^{3+} \downarrow=\mathrm{Fe}^{3+\uparrow}$. The resulting magnetization is $5 \mu_{\mathrm{B}}$ (recall $\mathrm{Y}$ does not have $f$ shell electrons) corresponding to $4 \pi M_{\mathrm{s}}=1750$ Gauss.

\subsection{Spinel Ferrites}

Spinel structure materials are closed packed cubic and have the form $\mathrm{AB}_{2} \mathrm{O}_{4}$ where $\mathrm{A}$ represents divalent cations and $\mathrm{B}$ trivalent cations. The exchange interaction between $\mathrm{A}$ and $\mathrm{B}$ sites is negative and the strongest among the cations so that the net magnetization results from the difference in magnetic moment between A and B sites. Spinel ferrites or ferrospinel are ferromagnetic materials with the general chemical composition 
Özgür et al., submitted to Journal of Materials Science: Materials in Electronics, 2009 Microwave Ferrites, Part 1: Fundamental properties

$\mathrm{MeO} \cdot \mathrm{Fe}_{2} \mathrm{O}_{3}$ where $\mathrm{Me}$ is a divalent metal such as iron, manganese, magnesium, nickel, zinc, cadmium, cobalt, copper, or a combination of these. The Fe ions are the trivalent variety, $\mathrm{Fe}^{3+}$. $\mathrm{MeO} \cdot \mathrm{Fe}_{2} \mathrm{O}_{3}$ has a structure identical to naturally occurring $\mathrm{MgAl}_{2} \mathrm{O}_{4}$ which goes by the name "spinel". Therefore, as mentioned above $\mathrm{MeO} \cdot \mathrm{Fe}_{2} \mathrm{O}_{3}$ like ferrites are called the ferrospinel or simply spinel.

As a group, spinel ferrites may be considered as derivatives of magnetite $\left(\mathrm{Fe}_{3} \mathrm{O}_{4}\right)$ contained in naturally occurring rocks. Here one $\mathrm{Fe}$ atom is trivalent $\mathrm{Fe}^{3+}$ and the other two are divalent $\mathrm{Fe}^{2+}$ ions. Magnetite has a cubic spinel structure with a lattice constant, $a_{0}$, of $8.39 \AA$. Magnetite contains, as mentioned, two ferric $\left(\mathrm{Fe}^{3+}\right)$ ions and one ferrous $\left(\mathrm{Fe}^{2+}\right)$ ion for each $\mathrm{O}^{2-}$ ions per molecule. Each neutral $\mathrm{Fe}$ atoms would have $63 d$ electrons, 4 of which are unpaired. Therefore, when participating in bonding, the trivalent $\mathrm{Fe}^{3+}$ utilizes two $4 s$ electrons and one $3 d$ electrons leading to 5 unpaired electrons in its $d$ shell. Somewhat similarly, divalent $\mathrm{Fe}^{2+}$ ions would have to have only the $4 s$ electrons participate in bonding leaving all its 6 electrons in the $3 d$ shell, 4 of which would be unpaired. Trivalent $\mathrm{Fe}^{3+}$ ions occupy all tetrahedral A sites and half of the octahedral B sites, while divalent $\mathrm{Fe}^{2+}$ ions occupy the other half of the octahedral B sites. In terms of magnetic properties, attributing 5 Bohr magnetons to $\mathrm{Fe}^{+3}$ (with 5 unpaired electrons), and 4 Bohr magnetons to $\mathrm{F}^{2+}$ (with 4 unpaired electrons) $\mathrm{Fe}_{3} \mathrm{O}_{4}$ would have a net of 4 Bohr magnetons. This is because the spinel structure contains one $\mathrm{Fe}^{3+}$ in the A site, and one $\mathrm{Fe}^{3+}$ and one $\mathrm{Fe}^{2+}$ in the $\mathrm{B}$ sites. $\mathrm{Fe}^{3+}$ ions at the octahedral sites contribute $-5 \mu_{B}$, $\mathrm{Fe}^{2+}$ ions at the tetragonal sites contribute $4 \mu_{B}$, and $\mathrm{Fe}^{3+}$ ions at the tetragonal sites contribute $5 \mu_{B}$ resulting in a net total magnetization of $4 \mu_{B}$ per molecular unit. 
The unit cell representing the smallest three-dimensional building block of the spinel crystal structure is shown in Figure 10 from which the cubic symmetry can be observed. The unit cell contains 32 oxygen anions (double negative charge for each totaling -64 ), 16 trivalent cations (triple positive charge for each totaling 48) and 8 divalent cations (double positive charge totaling 16) while maintaining the requisite charge balance for each unit cell. Due to two different valence cations available two types of crystallographic sites are present in the spinel structure. One is the tetrahedral " $\mathrm{A}$ " site surrounded by four oxygen ions while the other is the octahedral " $\mathrm{B}$ " site surrounded by six oxygen ions. The crystal structure described above for $\mathrm{Fe}_{3} \mathrm{O}_{4}$ represents an inverse spinel structure. Normal spinel arrangement requires that the eight divalent metal ions of the unit cell, i.e. $\mathrm{Me}^{2+}$ ions, occupy the $8 \mathrm{~A}$ sites and 16 trivalent metal ions, i.e. $\mathrm{Fe}^{3+}$ ions, occupy the 16 B sites. Further, there are two tetrahedral voids and one octahedral void for each $\mathrm{O}$ atom, i.e. 64 tetrahedral and 32 octahedral voids. Because there are only three metal ions per molecule in addition to $4 \mathrm{O}$ anions, only $1 / 4^{\text {th }}$ of the 12 voids are filled in a molecule. Moreover, divalent cation ( $\mathrm{Mg}$ in spinel) ions occupy only one-eight of the smaller tetrahedral voids, while trivalent cation fills one-half of the larger octahedral voids. In the simple and ideal fcc oxygen basis, the presence of cations in some voids causes shrinkage of the unoccupied voids, while maintaining the cubic overall symmetry. The lattice constant of ferrites is dependent on the metal-ion content, varying with different metal ions from a cube side of about $8.3 \AA$ to $8.5 \AA$. 
Özgür et al., submitted to Journal of Materials Science: Materials in Electronics, 2009 Microwave Ferrites, Part 1: Fundamental properties

\subsection{Hexaferrites}

Ferrites having a crystal structure similar to the mineral magnetoplumbite, $\mathrm{PbFe}_{7.5} \mathrm{Mn}_{3.5} \mathrm{Al}_{0.5} \mathrm{Ti}_{0.5} \mathrm{O}_{19}$ (without $\mathrm{Mn}$, Al, and $\mathrm{Ti}$ doping the chemical formula would be $\mathrm{PbO} \cdot 6 \mathrm{Fe}_{2} \mathrm{O}_{3}$ ), where the $\mathrm{Pb}$ ion is divalent, are referred to as the hexaferrites. Hexaferrites were in some ways born out of the need for coercive field (hard magnet) not provided by cubic ferrites such as the family of Alnico (considered to be soft particularly the early varieties). Typical coercivities are around $200 \mathrm{kA} / \mathrm{m}$. Moreover, they can easily be manufactured in powder form and shaped as desired.

The best known example of hexaferrites is Barium hexaferrite $\left(\mathrm{BaO} \cdot 6 \mathrm{Fe}_{2} \mathrm{O}_{3}\right.$ or $\left.\mathrm{BaFe}_{12} \mathrm{O}_{19}\right),{ }^{12}$ the trade names for which are Ferroxdure and Indox. ${ }^{13,14}$ The large $\mathrm{Ba}$ ion [sometimes replaced by $\mathrm{Sr}$ in Strontium hexaferrite $\left(\mathrm{SrO} \cdot 6 \mathrm{Fe}_{2} \mathrm{O}_{3}\right.$ or $\mathrm{SrFe}_{12} \mathrm{O}_{19}$ ], which causes a slight perturbation in the lattice around itself, is responsible for the large magnetic uniaxial anisotropy displayed by this group of ferrimagnets, where the crystallographic c-axis is the magnetic easy axis. This permanent magnetic material has a wide use of applications and can be found in every refrigerator door as well as innumerable dc motors. The manufacturing scale is huge with $50 \mathrm{~g}$ being produced for every person each year.

Several related ferromagnetic oxides are also available all of which can be derived by combining the ferrite spinel $\left(\mathrm{MeO} \cdot \mathrm{Fe}_{2} \mathrm{O}_{3}\right)$ and ferroxdure $\left(\mathrm{BaO} \cdot 6 \mathrm{Fe}_{2} \mathrm{O}_{3}\right)$ using the 
Özgür et al., submitted to Journal of Materials Science: Materials in Electronics, 2009 Microwave Ferrites, Part 1: Fundamental properties

chemical composition diagram shown in Figure 11. The same with chemical formula for each type, namely $\mathrm{M}, \mathrm{W}, \mathrm{Y}, \mathrm{Z}, \mathrm{U}$, and $\mathrm{X}$, is given in Table $5 .{ }^{15,16}$ For example, the chemical formulae for some $\mathrm{W}, \mathrm{Y}$, and $\mathrm{Z}$ hexaferrites are:

$\mathrm{Me}_{2} \mathbf{W}\left(\right.$ e.g. $\mathrm{Fe}_{2} \mathbf{W}: \mathrm{Ba}^{2+} \mathrm{Fe}_{2}^{2+} \mathrm{Fe}_{16}^{3+} \mathrm{O}_{27}$ )

$\mathrm{Me}_{2} \mathbf{Y}$ (e.g. $\mathrm{Mg}_{2} \mathbf{Y}: \mathrm{Ba}_{2}^{2+} \mathrm{Mg}_{2}^{2+} \mathrm{Fe}_{12}^{3+} \mathrm{O}_{22}$, Ferroxplana)

$\mathrm{Me}_{2} \mathbf{Z}\left(\right.$ e.g. $\left.\mathrm{Co}_{2} \mathbf{Z}: \mathrm{Ba}_{2}^{2+} \mathrm{Co}_{2}^{2+} \mathrm{Fe}_{24}^{3+} \mathrm{O}_{41}\right)$

As might be discerned from Table 5 the crystalline and magnetic structures of the different types of hexaferrites are remarkably complex, but all types are interrelated. (For some of the physical properties of hexaferrites of different types refer to Table 8. ${ }^{17,18,19,20,21,22,23,24,25,26,27,28}$ ) To elaborate further, utilizing the spinel structure $\mathrm{S}=$ $2 \mathrm{Me}^{2+} \mathrm{Fe}_{2} \mathrm{O}_{4}$ or $2 \mathrm{MeO} \cdot 2 \mathrm{Fe}_{2} \mathrm{O}_{3}$, (where $\mathrm{Me}=\mathrm{Fe}, \mathrm{Co}, \mathrm{Mn}, \mathrm{Ni}, \mathrm{Cu}, \mathrm{Mg}, \mathrm{Zn}$ representing the metal cation) and $\mathrm{M}=\mathrm{BaFe}_{12} \mathrm{O}_{19}$ or $\mathrm{BaO} \cdot 6 \mathrm{Fe}_{2} \mathrm{O}_{3}$, the W-type hexaferrite with chemical formula of $\mathrm{BaMe}_{2} \mathrm{Fe}_{16} \mathrm{O}_{27}$ can be represented as $\mathrm{W}=\mathrm{M}+\mathrm{S}$. The Y-type hexaferrite, $\mathrm{Y}=$ $\mathrm{Ba}_{2} \mathrm{Me}_{2} \mathrm{Fe}_{12} \mathrm{O}_{22}$, is a planar hexaferrite, and $\mathrm{Mg}_{2} \mathrm{Y}$ is known as Ferroxplana. Another planar hexaferrite, $3 \mathrm{BaO} \cdot 2 \mathrm{MeO} \cdot 12 \mathrm{Fe}_{2} \mathrm{O}_{3}$, can be constructed by $\mathrm{Z}=\mathrm{M}+\mathrm{Y}$. The X-type hexaferrite, $\mathrm{X}=2 \mathrm{M}+\mathrm{S}$, is formed by stacking of $\mathrm{R}$ - and $\mathrm{S}$-blocks along the hexagonal $c$ axis using the model RSR*S*S*, where R is an hexagonal three-oxygen-layer block with composition $\mathrm{BaFe}_{6} \mathrm{O}_{11}$, and the asterisk indicates that the corresponding block is rotated $180^{\circ}$ around the hexagonal axis. It can be seen that the structure of X-type compound is closely related to that of M- and W-type. The U-type hexaferrite can be constructed by $\mathrm{U}=2 \mathrm{M}+\mathrm{Y}$ as seen from the phase diagram in Figure 11. The crystalline structure of all types of hexaferrites is, therefore, what is termed as the mixed cubic-hexagonal structure. 
The hexagonal M-type ferrite, $\mathrm{BaO} \cdot 6 \mathrm{Fe}_{2} \mathrm{O}_{3}$, has lattice constants $a=5.89 \AA$ and $c=$ $23.19 \AA$ in an hexagonal closed packed lattice of oxygen and $\mathrm{Ba}$ with $\mathrm{Fe}$ in octahedral $\left(12 k, 4 f_{2}\right.$, and $\left.2 a\right)$, tetrahedral $\left(4 f_{1}\right)$, and trigonal bipyramidal/hexahedral $(2 b)$ sites. The Fe cations are the sole source of magnetic moment: the $12 k, 2 a$, and $2 b$ sites are spin up and $4 f_{1}$ and $4 f_{2}$ sites are spin down, as indicated in Figure 12, where the numbers in the site designations show total number of such sites in the unit cell.

The general structure of $\mathrm{M}$ type hexaferrite $\left(\mathrm{AO} \cdot 6 \mathrm{Fe}_{2} \mathrm{O}_{3}\right.$ or $\mathrm{AFe}_{12} \mathrm{O}_{19}$, where $\mathrm{A}$ is a divalent ion such as $\mathrm{Ba}^{2+}, \mathrm{Sr}^{2+}, \mathrm{Pb}^{2+}$, etc.), which is hexagonal with space group $P 63 / m m c$, is constructed from 4 building blocks, namely $\mathrm{S}, \mathrm{S}^{*}, \mathrm{R}$, and $\mathrm{R}^{*}$ as shown in Figure 12. The oxygen atoms are closed packed with the A and Fe ions in the interstitial sites. There are ten layers of oxygen atoms along the $c$ axis and the iron atoms are positioned at five crystallographically different sites. The $\mathrm{S}\left(\mathrm{Fe}_{6} \mathrm{O}_{8}\right)$ and $\mathrm{S}^{*}$ blocks are spinels with 2 oxygen layers and six $\mathrm{Fe}^{3+}$ ions. Four of these $\mathrm{Fe}^{3+}$ ions are in the octahedral sites with their spins aligned parallel to each other. The remaining two $\mathrm{Fe}^{3+}$ ions are in tetrahedral sites and have their spins antiparallel to those that are at the octahedral sites. As for the hexagonal $\mathrm{R}\left(\mathrm{AFe}_{6} \mathrm{O}_{11}\right)$ and $\mathrm{R}^{*}$ blocks, they consist of three oxygen layers with one of the oxygen anions replaced with an $\mathrm{A}$ ion $(\mathrm{A}=\mathrm{Ba}$ in Figure 12$)$. Each $\mathrm{R}$ block contains six $\mathrm{Fe}^{3+}$ ions, of which five are in octahedral sites, three having spin up and two having spin down polarization. In addition, one of the $\mathrm{Fe}^{3+}$ ions is coordinated with five $\mathrm{O}^{2-}$ anions and has spin up polarization. The Fe atoms at the $2 a$ site are octahedrally coordinated with equal $\mathrm{Fe}-\mathrm{O}$ distances, while the octahedrally coordinated Fe ions at $4 f_{2}$ 
Özgür et al., submitted to Journal of Materials Science: Materials in Electronics, 2009 Microwave Ferrites, Part 1: Fundamental properties

and $12 k$ sites have different $\mathrm{Fe}-\mathrm{O}$ interatomic distances, from about 1.85 to $2.37 \AA$. Of the twelve $\mathrm{Fe}^{3+}$ ions of the formula unit, the $\mathrm{Fe}$ atoms at $4 f_{1}$ sites are tetrahedrally coordinated by oxygen, while the Fe atoms at $2 b$ sites are coordinated by five oxygen ions. There are also short $\mathrm{Fe}-\mathrm{Fe}$ distances in the structure, and at $4 f_{2}$ sites this $\mathrm{Fe}-\mathrm{Fe}$ distance is about $2.7 \AA$ Á. The Fe ions at $12 k$ sites form a network with every Fe connected to four other Fe ions in the same layer. In terms of spin, in R block one ion in the $2 b$ layer is up state and two octahedral ions are down state, and in S block seven octahedral ions up state and two tetrahedral ions down state. Because each $\mathrm{Fe}^{3+}$ ion contributes $5 \mu_{\mathrm{B}}$ to the magnetic moment at absolute zero, the total magnetization at zero temperature can be calculated knowing that eight $\mathrm{Fe}^{3+}$ ions are in the spin up state, and four are in spin down state resulting in four net spin up $\mathrm{Fe}^{3+}$ ions. Therefore, the net magnetization per molecular unit is (1-2+7-2) $5 \mu_{\mathrm{B}}=20 \mu_{\mathrm{B}}$ (One Ba ion per molecular unit).

The $\mathrm{Me}_{2} \mathrm{~W}$ (W-type) hexaferrite also exhibits a magnetic uniaxial anisotropy along the caxis of the hexagonal structure as in the case of the M-type hexaferrite. The W structure consists of one Ba containing layer for every seventh oxygen layer of the spinel structure and is thus closely related to the M structure. Substitution of different combinations of divalent metal ions paves the way for changing the saturation magnetization and Néel temperature of the $\mathrm{W}$ hexaferrite. In a similar vein, both the $\mathrm{Me}_{2} \mathrm{Y}$ (Y-type) and $\mathrm{Me}_{2} \mathrm{Z}$ (Ztype) hexaferrites contain different combinations of barium layers and spinel blocks. However, the $\mathrm{Me}_{2} \mathrm{Y}$ has a preferred plane which is perpendicular to the $c$-axis in which the magnetization can move freely which is why $\mathrm{Me}_{2} \mathrm{Y}$ cannot be used as a permanent magnet. 
Özgür et al., submitted to Journal of Materials Science: Materials in Electronics, 2009 Microwave Ferrites, Part 1: Fundamental properties

Being the sum of M and Y compounds, the MeZ compound is somewhat different in that the $\mathrm{M}$ portion attempts to cause a preferential direction along the $c$-axis while the $\mathrm{Y}$ portion attempts to cause planar anisotropy in the plane perpendicular to the $c$-axis. The slightly larger uniaxial anisotropy in $\mathrm{M}$ prevails, however, and all $\mathrm{Me}_{2} \mathrm{Z}$ compounds with the exception of $\mathrm{Co}_{2} \mathrm{Z}$, which has planar anisotropy, are uniaxial.

\section{A primer to the theory of ferrimagnetism}

The theory of ferrimagnetism, which was developed by Néel, ${ }^{29,30}$ is similar to that of antiferromagnetism except that the opposing magnetization of two sublattices A and B in a ferrimagnetic material are not equal while they are in an antiferromagnetic material. This implies that there is a net magnetization in ferrimagnetic materials but not in antiferromagnetic materials, as discussed in Section 2.1. The properties of ferrimagnets can be described through $\mathrm{A}-\mathrm{A}, \mathrm{B}-\mathrm{B}$, and $\mathrm{A}-\mathrm{B}$ exchange interaction and through the magnetization of each sublattice. Magnetization requires the alignment of magnetic moments of atoms. This typically is a result of exchange interaction between the magnetic ions and the host. One method used to describe this assumes that as if there is an internal field, called the exchange field, which causes their alignment changing the state of the material from paramagnetic to ferromagnetic or ferrimagnetic. The effect of exchange field is opposed by thermal agitations and above a certain temperature, which is the Curie temperature, ferromagnetism is destroyed. Consideration of the exchange field, mean field, goes by the name "mean field theory" as already mentioned above. 
In the mean field theory, to characterize $\mathrm{A}-\mathrm{A}, \mathrm{B}-\mathrm{B}$, and $\mathrm{A}-\mathrm{B}$ exchange interactions molecular-field coefficients $\lambda_{E, a a}, \lambda_{E, b b}$, and $\lambda_{E, a b}=\lambda_{E, b a}$ are introduced (compared to a single coefficient $\lambda_{E}$ in Equation 13), and these coefficients may have negative or positive signs. We should keep in mind, however, that for $T>T_{C}$ (the Curie temperature) the system is a disordered paramagnet, and for $T<T_{C}$ the system is a ferromagnet or a ferrimagnet. To reiterate, in a ferromagnetic material the magnetic moments associated with all the atoms are aligned in the same direction. In a ferrimagnetic material, however, magnetic moment of sublattice A and that of sublattice B are in opposite direction but with unequal amplitudes, which gives rise to non zero net magnetization. The total magnetic field in the absence of external magnetic field acting on a magnetic dipole in each sublattice is (in cgs units)

$$
\begin{aligned}
& H_{a}=\lambda_{E, a a} M_{a}+\lambda_{E, a b} M_{b} \\
& H_{b}=\lambda_{E, a b} M_{a}+\lambda_{E, b b} M_{b}
\end{aligned}
$$

\section{Equation 14}

The magnetization of each sublattice can be described by the Curie relations where they have their own Curie constants $C_{a}$ and $C_{b}$, which are not identical since each sublattice contains different kinds of ions on different crystallographic sites, as

$$
\begin{aligned}
& M_{a}=\frac{C_{a}}{T}\left(H_{0}+H_{a}\right) \\
& M_{b}=\frac{C_{b}}{T}\left(H_{0}+H_{b}\right)
\end{aligned}
$$

\section{Equation 15}


Özgür et al., submitted to Journal of Materials Science: Materials in Electronics, 2009 Microwave Ferrites, Part 1: Fundamental properties

Solving Equation 14 and Equation 15, the inverse susceptibility of a ferrimagnet in the paramagnetic regime can be expressed as follows:

$$
\frac{1}{\chi}=\frac{H_{0}}{M_{a}+M_{b}}=\frac{T}{C}+\frac{1}{\chi_{0}}-\frac{K}{T-\Theta^{\prime}} \quad \text { in cgs units }
$$

\section{Equation 16}

where $1 / \chi_{0}, K, C$, and $\Theta^{\prime}$ are constants depending on $C_{a}, C_{b}, \lambda_{E, a a}, \lambda_{E, b b}$, and $\lambda_{E, a b}$.

\subsection{Ferromagnetic resonance and the permeability tensor}

Before delving into the theory of the permeability tensor in ferrites, it is imperative that we say a few words about magnetostriction which goes to the heart of electrical modulation of magnetic property. If a ferromagnetic material were to be strained by applying a suitable stress along a crystal direction, the interatomic distance along this direction as well as those along the other two orthogonal directions would change. Consequently the exchange interaction of the atomic spins would change, thereby changing the magnetization of the ferromagnetic or ferrimagnetic material. The converse is also true in that if a magnetic field is applied, the interatomic distance would change. When an Fe crystal is placed in a magnetic field along an easy direction [100], the crystal gets dilated in this direction and contracts in the transverse directions, [010] and [001]. The longitudinal strain $\Delta l / l$ along the direction of magnetization is proportional to the magnetostrictive constant, often denoted by $\lambda$ (not to be confused with the molecular field

coefficient we will denote it by $\left.\lambda^{(M S)}\right)$. This constant depends on the crystal direction and it can be positive (extension - tensile) or negative leading to physical contraction (compression). Moreover, $\lambda^{(M S)}$ is a function of the magnetic field and it can even change 
Özgür et al., submitted to Journal of Materials Science: Materials in Electronics, 2009 Microwave Ferrites, Part 1: Fundamental properties

its sign as the field is increased. A case in point is Fe. Furthermore, when the crystal reaches the saturation magnetization, $\lambda^{(M S)}$ also reaches a saturation called the saturation magnetostrictive strain (or magnetostriction), $\lambda_{s}^{(M S)}$. The crystal lattice strain energy associated with magnetostriction is termed as the magnetostrictive energy, which is typically smaller than the anisotropy energy. On the practical side, transformer hum noise, with which most of us would be familiar, is a result of magnetostrictive effect. Changing the magnetic field direction causes the alternating longitudinal strain to vibrate the surrounding.

The theory of ferromagnetic resonance has been discussed extensively in several books. ${ }^{31,32,33,34}$ Here a brief discussion of the basic theory will be provided. To understand the ferromagnetic resonance (FMR) one should first consider the torque exerted on a magnetic dipole by the magnetic field $\mathbf{H}$. This torque is expressed by

$$
\mathbf{T}=\mu_{0} \mathbf{m} \times \mathbf{H}=-\mu_{0} \gamma \mathbf{S} \times \mathbf{H}
$$

\section{Equation 17}

where $\mu_{0}$ is the permeability of vacuum, $\mathbf{S}$ (used for consistency with material presented above even though $\mathbf{s}$ is typically used for single particles) is the spin angular momentum that is opposite to the dipole magnetic moment $\mathbf{m}$ for the electron, and $\gamma=-\mathrm{m} / \mathrm{S}$ is the gyromagnetic ratio defined before. The torque acting on a body, see Figure 13 for a pictorial view, is equal to the rate of change of angular momentum of the body:

$$
\mathbf{T}=\frac{d \mathbf{S}}{d t}=\frac{-1}{\gamma} \frac{d \mathbf{m}}{d t}=\mu_{0} \mathbf{m} \times \mathbf{H}
$$

\section{Equation 18}


Özgür et al., submitted to Journal of Materials Science: Materials in Electronics, 2009 Microwave Ferrites, Part 1: Fundamental properties

Under a strong enough static magnetic field, the magnetization of the material is assumed to be saturated and the total magnetization vector is given by $\mathbf{M}=N \mathbf{m}$, where $\mathrm{N}$ is the effective number of dipoles per unit volume. We can then obtain the macroscopic equation of motion of the magnetization vector from Equation 18 as

$$
\frac{d \mathbf{M}}{d t}=-\mu_{0} \gamma \mathbf{M} \times \mathbf{H}
$$

\section{Equation 19}

The minus sign is due to the negative charge of the electron that is carried out from $\gamma$. In many cases the absolute value of the electronic charge is used for practical purposes and the minus sign is removed (That's why the torque in Figure 13 is shown in the direction of $-\mathbf{m} \times \mathbf{H}) . \mathbf{H}$ is the vector sum of all fields, external and internal, acting upon the magnetization and includes the DC field $\mathbf{H}_{\mathbf{i}}$ and the RF field $\mathbf{H}_{\mathrm{ac}}$. Because $\mathrm{H}_{\mathrm{ac}}$ is much smaller than $\mathrm{H}_{\mathrm{i}}$ there is a linear relationship between $\mathbf{H}$ and $\mathbf{M}$. The actual relationship between the magnetic intensity $\mathbf{H}$, which is averaged over the space for many molecules within the magnetic material, and the external applied field depends upon the shape of the ferrite body. ${ }^{32} \mathbf{H}_{\mathbf{i}}$ is the vector sum of all DC fields within the material including that due to the externally applied DC field $\mathbf{H}_{\mathbf{0}}$ and any time-independent internal fields. For simplicity, we will assume that the medium is infinite, i.e. there is no demagnetization correction that depends on the shape of the material, the crystal anisotropy or magnetostriction is zero, and only the externally applied DC field $\mathbf{H}_{\mathbf{0}}$ contributes to the total internal field $\mathbf{H}_{\mathbf{i}}$, and it is large enough to saturate the magnetization. The total magnetic field can be expressed as 
Özgür et al., submitted to Journal of Materials Science: Materials in Electronics, 2009 Microwave Ferrites, Part 1: Fundamental properties

$$
\mathbf{H}=\mathbf{H}_{\mathbf{0}}+\mathbf{H}_{\mathrm{ac}}
$$

\section{Equation 20}

The resulting magnetization will then have the form

$$
\mathbf{M}=\mathbf{M}_{\mathbf{s}}+\mathbf{M}_{\mathbf{a c}}
$$

\section{Equation 21}

where $\mathbf{M}_{\mathbf{s}}$ is the DC saturation magnetization and $\mathbf{M}_{\mathbf{a c}}$ is the $\mathrm{AC}$ magnetization. In Equation 21, we have assumed that the $\mathbf{H}$ field is strong enough to drive the magnetization into saturation, which is true for practical applications. The DC equation can be obtained from Equation 19 by neglecting all the AC terms:

$$
\mu_{0} \gamma \mathbf{M}_{\mathbf{s}} \times \mathbf{H}_{\mathbf{0}}=0
$$

\section{Equation 22}

which indicates that, to the first order, the internal DC field $\left(\mathbf{H}_{\mathbf{i}}\right.$ if contributions other than $\mathbf{H}_{\mathbf{0}}$ are included) and the magnetization are in the same direction. Substituting Equation 20 and Equation 21 into Equation 19 (removing the minus sign) and taking into account $d \mathbf{M}_{\mathrm{s}} / d t=0$, we obtain

$$
\frac{d \mathbf{M}_{\mathrm{ac}}}{d t}=\mu_{0} \gamma\left(\mathbf{M}_{\mathrm{s}} \times \mathbf{H}_{\mathrm{ac}}+\mathbf{M}_{\mathrm{ac}} \times \mathbf{H}_{0}\right)
$$

\section{Equation 23}

The products of the AC terms (second order terms) are neglected in Equation 23 since the magnitudes of the AC components are much smaller than those of the DC components. Further assuming that the $\mathrm{AC}$ field, and therefore the $\mathrm{AC}$ magnetization, have a harmonic time dependence, $e^{j \omega t}$, and by arbitrarily choosing the applied DC field direction along 
Özgür et al., submitted to Journal of Materials Science: Materials in Electronics, 2009 Microwave Ferrites, Part 1: Fundamental properties

the $z$-axis (therefore, the RF field is in the $x-y$ plane) the components of Equation 23 in the Cartesian coordinate system can be written as

$$
j \omega M_{x}=\omega_{0} M_{y}-\omega_{m} H_{y}
$$

\section{Equation 24}

$$
j \omega M_{y}=-\omega_{0} M_{x}+\omega_{m} H_{x}
$$

Equation 25

$$
j \omega M_{z}=0
$$

Equation 26

where

$$
\omega_{0}=\mu_{0} \gamma H_{0}, \omega_{m}=\mu_{0} \gamma M_{s}
$$

\section{Equation 27}

$\omega_{0}$ is called the Larmor frequency, which defines the natural precession frequency under a static magnetic field (see Figure 13 for the precession motion). Solving Equation 24 and Equation 25 for $M_{x}$ and $M_{y}$ gives $\left(M_{z}=0\right.$ from Equation 26) :

$$
M_{x}=\frac{\omega_{0} \omega_{m}}{\left(\omega_{0}^{2}-\omega^{2}\right)} H_{x}-\frac{j \omega \omega_{m}}{\left(\omega_{0}^{2}-\omega^{2}\right)} H_{y}
$$

\section{Equation 28}

$$
M_{y}=\frac{j \omega \omega_{m}}{\left(\omega_{0}^{2}-\omega^{2}\right)} H_{x}+\frac{\omega_{0} \omega_{m}}{\left(\omega_{0}{ }^{2}-\omega^{2}\right)} H_{y}
$$

\section{Equation 29}

These solutions can be written in tensor form:

\section{Equation 30}

$$
\mathbf{M}_{\mathbf{a c}}=\left[\chi_{a c}\right] \mathbf{H}_{\mathbf{a c}}=\left[\begin{array}{ccc}
\chi_{x x} & \chi_{x y} & 0 \\
\chi_{y x} & \chi_{y y} & 0 \\
0 & 0 & 0
\end{array}\right]\left[\begin{array}{l}
H_{x} \\
H_{y} \\
H_{z}
\end{array}\right]
$$


Özgür et al., submitted to Journal of Materials Science: Materials in Electronics, 2009 Microwave Ferrites, Part 1: Fundamental properties

where $[\chi]$ is the dynamic susceptibility tensor. Notice that for the given configuration (applied field in the $z$-direction) the total magnetization can be written as

$$
\mathbf{M}=\mathbf{M}_{s}+\mathbf{M}_{\mathbf{a c}}=\left[\begin{array}{c}
M_{x} \\
M_{y} \\
M_{s}
\end{array}\right]=\left[\begin{array}{ccc}
\chi_{x x} & \chi_{x y} & 0 \\
\chi_{y x} & \chi_{y y} & 0 \\
0 & 0 & \chi_{0}
\end{array}\right]\left[\begin{array}{c}
H_{x} \\
H_{y} \\
H_{0}
\end{array}\right]=[\chi]\left(\mathbf{H}_{\mathbf{0}}+\mathbf{H}_{\mathbf{a c}}\right)
$$

\section{Equation 31}

where $\chi_{0}=H_{0} / M_{s}$. Comparing Equation 30 with Equation 28 and Equation 29, the elements of $[\chi]$ are obtained as

$$
\chi_{x x}=\chi_{y y}=\frac{\omega_{0} \omega_{m}}{\omega_{0}^{2}-\omega^{2}}
$$

\section{Equation 32}

$$
\chi_{x y}=-\chi_{y x}=-\frac{j \omega \omega_{m}}{\omega_{0}^{2}-\omega^{2}}
$$

\section{Equation 33}

One can now derive the permeability tensor $[\mu]$ using

$$
\mathbf{B}=\mu_{0}(\mathbf{M}+\mathbf{H})=\mu_{0}(\mathbf{I}+[\chi]) \mathbf{H}=[\mu] \mathbf{H}
$$

\section{Equation 34}

The conventional notation (referred to as the Polder tensor ${ }^{35}$ ) for the permeability tensor for a magnetic field bias along the z-direction is

$$
[\mu]=\left[\begin{array}{ccc}
\mu & -j \kappa & 0 \\
j \kappa & \mu & 0 \\
0 & 0 & \mu_{0}
\end{array}\right]
$$

\section{Equation 35}

where

$$
\mu=\mu_{0}\left(1+\chi_{x x}\right)=\mu_{0}\left(1+\chi_{y y}\right)=\mu_{0}\left(1+\frac{\omega_{0} \omega_{m}}{\omega_{0}^{2}-\omega^{2}}\right)
$$

\section{Equation 36}


Özgür et al., submitted to Journal of Materials Science: Materials in Electronics, 2009 Microwave Ferrites, Part 1: Fundamental properties

Equation 37

$$
j \kappa=-\mu_{0} \chi_{x y}=\mu_{0} \chi_{y x}=j \mu_{0} \frac{\omega \omega_{m}}{\omega_{0}^{2}-\omega^{2}}
$$

As shown in Equation 35 the permeability tensor is antisymmetric owing to $x y$ and $y x$ terms having opposite signs, unlike the conductivity and permittivity tensors. Such antisymmetrical condition of ferrites is necessary for nonreciprocal devices (as will be discussed in Section 3 in Part 2). ${ }^{3}$ Obviously, the permeability tensor has a singularity at $\omega=\omega_{0}$, which is known as the ferromagnetic (or gyromagnetic) resonance. When the driving frequency $\omega$ is equal to the natural precession frequency $\omega_{0}$ the energy from the microwave field is transferred most efficiently to the system of spins. At $\omega \neq \omega_{0}$ there is an oscillating component of the magnetization superimposed on the steady-state precession and the nearer the frequency of the microwave field to the natural precession frequency, the greater the energy absorbed by the spins will be. To observe this resonance, either the frequency or the applied DC field $H_{0}$ can be swept until the precession frequency equals the microwave frequency.

\subsection{Damped ferromagnetic resonance and Losses}

In the derivations so far, the losses associated with the dipole motion in an actual ferromagnetic (or ferrimagnetic) medium are neglected for simplicity. In reality, however, all ferrimagnetic materials have losses that damp out the resonance, where the damping forces oppose the precessional motion to relax the magnetization back to the steady-state equilibrium (see Figure 13 for a pictorial view). To account for the losses, a damping coefficient is introduced into the equation of motion and the range of static magnetic field (at a fixed frequency) over which the absorption is significant, is denoted by the 
Özgür et al., submitted to Journal of Materials Science: Materials in Electronics, 2009 Microwave Ferrites, Part 1: Fundamental properties

linewidth. The loss has been represented phenomenologically in the equations of motion by two main forms: ${ }^{36}$ the Bloch-Bloembergen ${ }^{37}$ and the Landau-Lifshitz ${ }^{38}$ forms. Since it can be introduced simply into the susceptibility equations and represents the overall losses adequately, the Landau-Lifshitz form will be considered here. The original representation of the Landau-Lifshitz equation of motion of the magnetization is (adding the loss term to Equation 19 and removing the minus sign due to the electron charge for practical purposes)

$$
\frac{d \mathbf{M}}{d t}=\gamma \mu_{0}(\mathbf{M} \times \mathbf{H})-\frac{\xi \gamma}{M^{2}} \mu_{0} \mathbf{M} \times(\mathbf{M} \times \mathbf{H})
$$

\section{Equation 38}

where $\xi$ is a dissipation parameter. Equation 38 can be written in the form proposed by Gilbert: ${ }^{39}$

$$
\begin{aligned}
\frac{d \mathbf{M}}{d t} & =\gamma \mu_{0}(\mathbf{M} \times \mathbf{H})-\frac{\xi \gamma}{M^{2}} \mu_{0} \mathbf{M} \times \frac{1}{\gamma \mu_{0}}\left\{\frac{d \mathbf{M}}{d t}+\frac{\xi \gamma}{M^{2}} \mu_{0} \mathbf{M} \times(\mathbf{M} \times \mathbf{H})\right\} \\
& =\gamma \mu_{0}(\mathbf{M} \times \mathbf{H})\left(1+\frac{\xi^{2}}{M^{2}}\right)-\frac{\xi}{M^{2}} \mathbf{M} \times \frac{d \mathbf{M}}{d t} \\
& =\gamma^{*} \mu_{0}(\mathbf{M} \times \mathbf{H})-\frac{\phi}{|M|} \mathbf{M} \times \frac{d \mathbf{M}}{d t}
\end{aligned}
$$

\section{Equation 39}

where $\phi=\xi /|M|$ is a dimensionless damping factor and $\gamma^{*}=\gamma\left(1+\phi^{2}\right)$. The LandauLifshitz and Gilbert forms are nearly identical when $\phi<<1$. Solving Equation 39 using Equation 20 and Equation 21 with $e^{j \omega t}$ time dependence as we have done for Equation 19, one can find that the only difference when the losses are taken into account is that $\omega_{0}$ in 
Özgür et al., submitted to Journal of Materials Science: Materials in Electronics, 2009 Microwave Ferrites, Part 1: Fundamental properties

Equation 32 and Equation 33 is replaced with a complex angular frequency $\omega_{0}+j \phi \omega$.

After this substitution we obtain the complex susceptibilities

Equation 40

$$
\chi_{x x}=\chi_{x x}^{\prime}-j \chi_{x x}^{\prime \prime}
$$

$$
\chi_{x y}=-\chi_{x y}^{\prime \prime}-j \chi_{x y}^{\prime}
$$

\section{Equation 41}

where the real and imaginary parts are given by

\section{Equation 42}

$$
\chi_{x x}^{\prime}=\frac{\omega_{0} \omega_{m}\left(\omega_{0}^{2}-\omega^{2}\right)+\omega_{0} \omega_{m} \omega^{2} \phi^{2}}{\left[\omega_{0}^{2}-\omega^{2}\left(1+\phi^{2}\right)\right]^{2}+4 \omega_{0}^{2} \omega^{2} \phi^{2}}
$$

$$
\chi_{x x}^{\prime \prime}=\frac{\phi \omega \omega_{m}\left[\omega_{0}^{2}+\omega^{2}\left(1+\phi^{2}\right)\right]}{\left[\omega_{0}^{2}-\omega^{2}\left(1+\phi^{2}\right)\right]^{2}+4 \omega_{0}^{2} \omega^{2} \phi^{2}}
$$

Equation 43

$$
\chi_{x y}^{\prime}=\frac{\omega_{0} \omega_{m}\left[\omega_{0}^{2}-\omega^{2}\left(1+\phi^{2}\right)\right]}{\left[\omega_{0}^{2}-\omega^{2}\left(1+\phi^{2}\right)\right]^{2}+4 \omega_{0}^{2} \omega^{2} \phi^{2}}
$$

\section{Equation 44}

$$
\chi_{x y}^{\prime \prime}=\frac{2 \omega_{0} \omega_{m} \omega^{2} \phi}{\left[\omega_{0}^{2}-\omega^{2}\left(1+\phi^{2}\right)\right]^{2}+4 \omega_{0}^{2} \omega^{2} \phi^{2}}
$$

\section{Equation 45}

The definition in the equations above for the real and imaginary parts of $\chi_{x y}$ is intentionally reversed compared to $\chi_{x x}$ to reflect the fact that $\mu_{0} \chi_{x y}=-j \kappa$ as in Equation 37.

Figure 14 shows the real and imaginary (i.e. dispersive and dissipative) components of the susceptibility tensor elements as a function of the applied magnetic field. The damping factor can be computed from the experimental linewidth $\Delta H$ of the absorption 
Özgür et al., submitted to Journal of Materials Science: Materials in Electronics, 2009 Microwave Ferrites, Part 1: Fundamental properties

curve near resonance, which is the linewidth of the $\chi_{x x}^{\prime \prime} v s . H_{0}$ curve where $\chi_{x x}^{\prime \prime}$ decreases to half of its peak value. For a fixed frequency, resonance occurs when $H_{0}=H_{r}$ so that $\omega_{0}$ $=\omega_{r}=\mu_{0} \gamma H_{r}$ (special case when there is no shape anisotropy, e.g. for a spherical of infinite medium).

Taking into account that $\phi<<1$, and therefore, $1+\phi^{2} \approx 1$, one can obtain the linewidth from Equation 43 as (again in a medium with no shape anisotropy)

$$
\Delta H=\frac{\Delta \omega}{\mu_{0} \gamma}=\frac{2 \phi \omega_{0}}{\mu_{0} \gamma}=2 \phi H_{0}
$$

\section{Equation 46}

A more general form of the linewidth can be obtained by considering the total energy per unit volume, $E$, of the magnetic system in spherical coordinates, which has contributions from the external field, demagnetization, magnetic anisotropy, magneto-elastic energy, exchange energy, and the inter-domain boundary layers. ${ }^{33}$ Let's first consider the lossless case. Using a constant magnetization $\mathbf{M}$ having components $M_{x}=M \sin \theta \cos \varphi$, $M_{y}=M \sin \theta \sin \varphi$, and $M_{z}=M \cos \theta$ ( $M$ is constant), and writing the radial $\left(H_{M}\right)$, polar $\left(H_{\theta}\right)$, and azimuthal $\left(H_{\varphi}\right)$ components of $\mathrm{H}$ as (radial component is along $\left.\mathbf{M}\right)$

$$
\begin{aligned}
& H_{M}=H_{x} \sin \theta \cos \varphi+H_{y} \sin \theta \sin \varphi+H_{z} \cos \theta \\
& H_{\theta}=H_{x} \cos \theta \cos \varphi+H_{y} \cos \theta \sin \varphi-H_{z} \sin \theta \\
& H_{\varphi}=-H_{x} \sin \varphi+H_{y} \cos \varphi
\end{aligned}
$$

\section{Equation 47}

the equation of motion (Equation 19) takes the form 
Özgür et al., submitted to Journal of Materials Science: Materials in Electronics, 2009 Microwave Ferrites, Part 1: Fundamental properties

$$
\frac{d \theta}{d t}=\mu_{0} \gamma H_{\varphi} \quad ; \quad \frac{d \varphi}{d t} \sin \theta=-\mu_{0} \gamma H_{\theta}
$$

\section{Equation 48}

At thermodynamic equilibrium, the magnetization and the magnetic field point at the same direction, i.e. the components $H_{\theta}$ and $H_{\varphi}$ are zero. $H_{M}$ is determined using the total energy from

$$
H_{M}=-\frac{\partial E}{\partial M}
$$

\section{Equation 49}

In a homogeneous system, the equilibrium orientation of the magnetization vector $\mathbf{M}$, i.e. the equilibrium angle values $\theta_{0}$ and $\varphi_{0}$, can be determined from $\partial E / \partial \theta=\partial E / \partial \varphi=0$ minimizing the total energy. In the non-equilibrium case where there are small deviations in the magnetization from the equilibrium position, the magnetic field components perpendicular to the magnetization direction will be nonzero:

$$
H_{\theta}=-\frac{1}{M} \frac{\partial E}{\partial \theta} \quad ; \quad H_{\varphi}=-\frac{1}{M \sin \theta} \frac{\partial E}{\partial \varphi}
$$

\section{Equation 50}

For small deviations from the equilibrium values $\theta_{0}$ and $\varphi_{0}$, i.e. for small $\delta \theta(t)=\theta(t)-\theta_{0}$ and $\delta \varphi(t)=\varphi(t)-\varphi_{0}$ one can consider only the linear terms in the expansions of the first derivatives of the total energy:

$$
\frac{\partial E}{\partial \theta}=\frac{\partial^{2} E}{\partial \theta^{2}} \delta \theta+\frac{\partial^{2} E}{\partial \theta \partial \varphi} \delta \varphi \quad ; \quad \frac{\partial E}{\partial \varphi}=\frac{\partial^{2} E}{\partial \varphi \partial \theta} \delta \theta+\frac{\partial^{2} E}{\partial \varphi^{2}} \delta \varphi
$$

\section{Equation 51}


Özgür et al., submitted to Journal of Materials Science: Materials in Electronics, 2009 Microwave Ferrites, Part 1: Fundamental properties

Using Equation 48, Equation 50, and Equation 51 one can then obtain a system of linear equations describing the small oscillations of the magnetization vector about the equilibrium position:

$$
\begin{gathered}
-\frac{1}{\mu_{0} \gamma} M \sin \theta_{0} \frac{d \delta \theta}{d t}=\frac{\partial^{2} E}{\partial \varphi \partial \theta} \delta \theta+\frac{\partial^{2} E}{\partial \varphi^{2}} \delta \varphi \\
\frac{1}{\mu_{0} \gamma} M \sin \theta_{0} \frac{d \delta \varphi}{d t}=\frac{\partial^{2} E}{\partial \theta^{2}} \delta \theta+\frac{\partial^{2} E}{\partial \theta \partial \varphi} \delta \varphi
\end{gathered} .
$$

\section{Equation 52}

For time varying fields, Equation 52 has periodic solutions, i.e. $\delta \theta, \delta \varphi \sim \exp (j \omega t)$. The characteristic (resonant) frequency of oscillations, $\omega_{r}$, is obtained by setting the determinant of the homogeneous system of equations (Equation 52) to zero:

$$
\left(\frac{\partial^{2} E}{\partial \theta \partial \varphi}\right)^{2}-\frac{\partial^{2} E}{\partial \theta^{2}} \frac{\partial^{2} E}{\partial \varphi^{2}}+\frac{\omega^{2}}{\mu_{0}^{2} \gamma^{2}} M^{2} \sin ^{2} \theta_{0}=0,
$$

\section{Equation 53}

which gives

$$
\omega_{r}=\frac{\mu_{0} \gamma}{M \sin \theta_{0}}\left\{\frac{\partial^{2} E}{\partial \theta^{2}} \frac{\partial^{2} E}{\partial \varphi^{2}}-\left(\frac{\partial^{2} E}{\partial \theta \partial \varphi}\right)^{2}\right\}^{1 / 2} .
$$

\section{Equation 54}

In the lossy case, one needs to start from the equation of motion including loss, Equation 38, which after using Equation 47 takes the form:

$$
\frac{d \theta}{d t}=\mu_{0} \gamma\left(H_{\varphi}+\phi H_{\theta}\right) \quad ; \quad \frac{d \varphi}{d t} \sin \theta=-\mu_{0} \gamma\left(H_{\theta}-\phi H_{\varphi}\right) .
$$

\section{Equation 55}


Özgür et al., submitted to Journal of Materials Science: Materials in Electronics, 2009 Microwave Ferrites, Part 1: Fundamental properties

The system of linear equations given describing the small oscillations of the magnetization vector about the equilibrium position are in the lossy case given as

$$
\begin{gathered}
-\frac{1}{\mu_{0} \gamma} M \sin \theta_{0} \frac{d \delta \theta}{d t}=\left(\frac{\partial^{2} E}{\partial \varphi \partial \theta}+\phi \sin \theta_{0} \frac{\partial^{2} E}{\partial \theta^{2}}\right) \delta \theta+\left(\frac{\partial^{2} E}{\partial \varphi^{2}}+\phi \sin \theta_{0} \frac{\partial^{2} E}{\partial \theta \partial \varphi}\right) \delta \varphi \\
\frac{1}{\mu_{0} \gamma} M \sin \theta_{0} \frac{d \delta \varphi}{d t}=\left(\frac{\partial^{2} E}{\partial \theta^{2}}-\frac{\phi}{\sin \theta_{0}} \frac{\partial^{2} E}{\partial \theta \partial \varphi}\right) \delta \theta+\left(\frac{\partial^{2} E}{\partial \theta \partial \varphi}-\frac{\phi}{\sin \theta_{0}} \frac{\partial^{2} E}{\partial \varphi^{2}}\right) \delta \varphi
\end{gathered}
$$

\section{Equation 56}

Note that Equation 56 transforms into Equation 52 when damping is zero $(\phi=0)$.

Equation 56 has periodic solutions with the frequency satisfying

$$
\omega^{2}-j \omega \Delta \omega-\omega_{r}^{2}=0
$$

\section{Equation 57}

where the resonance frequency is

$$
\omega_{r}=\frac{\mu_{0} \gamma\left(1+\phi^{2}\right)^{1 / 2}}{M \sin \theta_{0}}\left\{\frac{\partial^{2} E}{\partial \theta^{2}} \frac{\partial^{2} E}{\partial \varphi^{2}}-\left(\frac{\partial^{2} E}{\partial \theta \partial \varphi}\right)^{2}\right\}^{1 / 2},
$$

\section{Equation 58}

and the width of the resonant absorption line is

$$
\Delta \omega \equiv\left(\frac{d \omega}{d H}\right) \Delta H=\frac{\phi \mu_{0} \gamma}{M}\left(\frac{\partial^{2} E}{\partial \theta^{2}}+\frac{1}{\sin ^{2} \theta_{0}} \frac{\partial^{2} E}{\partial \varphi^{2}}\right),
$$

\section{Equation 59}

The magnetic field resonance linewidth is then

$$
\Delta H=\frac{1}{d \omega / d H} \frac{\phi \mu_{0} \gamma}{M}\left(\frac{\partial^{2} E}{\partial \theta^{2}}+\frac{1}{\sin ^{2} \theta_{0}} \frac{\partial^{2} E}{\partial \varphi^{2}}\right) .
$$


Özgür et al., submitted to Journal of Materials Science: Materials in Electronics, 2009 Microwave Ferrites, Part 1: Fundamental properties

\section{Equation 60}

It should be noted here that to find the resonant frequency and the linewidth of a ferromagnetic crystal it is necessary to have a concrete expression of its total energy per unit volume, which depends on the orientation of the magnetization.

For direct measurements of the susceptibility tensor elements and to understand the operational principles of the Faraday rotators (see Section 2.1 in Part 2) ${ }^{3}$, it is convenient to represent the susceptibility in terms of the response to two circularly polarized microwave fields with opposite helicity. In this case, the effective susceptibility can be written as

$$
\chi_{ \pm}=\chi_{x x} \pm \chi_{x y}
$$

\section{Equation 61}

where + and - are used for opposite helicities. After substituting Equation 40 and Equation 41 into Equation 61 the real and imaginary parts of $\chi_{ \pm}=\chi_{ \pm}^{\prime}-j \chi_{ \pm}^{\prime \prime}$ are obtained as

$$
\chi_{ \pm}^{\prime}=\frac{\omega_{m}\left(\omega_{0} \mp \omega\right)}{\left(\omega_{0} \mp \omega\right)^{2}+\phi^{2} \omega^{2}}
$$

Equation 62

$$
\chi_{ \pm}^{\prime \prime}=\frac{\phi \omega \omega_{m}}{\left(\omega_{0} \mp \omega\right)^{2}+\phi^{2} \omega^{2}}
$$

\section{Equation 63}


Özgür et al., submitted to Journal of Materials Science: Materials in Electronics, 2009 Microwave Ferrites, Part 1: Fundamental properties

The positive helicity susceptibility $\chi_{+}$, which corresponds to the circularly polarized wave that rotates in the same direction as the precessional motion, has a singularity at $\omega=\omega_{0}$. The negative helicity susceptibility $\chi$, however, does not have any singularity and no resonance can be achieved for a circularly polarized magnetic field rotating opposite to the precession.

The resonance frequency in a real body is influenced by other loss mechanisms associated with demagnetization, magnetic anisotropy, and crystalline imperfections (polycrystal structure). The susceptibility has been derived above for an infinite medium to simply illustrate the fundamentals. Obviously, in the real case the samples are finite and the magnetic fields inside the sample should be evaluated by solving the electromagnetic boundary value problem. To make the problem more convenient, the sample may be assumed to be small compared to the wavelength leading to a uniform microwave magnetic field inside the sample; however, the internal field is not equal to the field outside the sample due to the discontinuity of the normal component of the susceptibility at the surface.

\subsection{Demagnetization}

In the discussions of the microwave susceptibility so far, the medium has been assumed to be infinite. However, one needs to evaluate the magnetic fields in samples of finite size, and demagnetization effects arise for finite samples. The analysis of these effects is simplified for uniformly shaped samples (sphere, cylinder, thin slab, etc.), for which the 
Özgür et al., submitted to Journal of Materials Science: Materials in Electronics, 2009 Microwave Ferrites, Part 1: Fundamental properties

demagnetization factors can be easily obtained. Also for samples smaller than the wavelength the microwave magnetic field inside the sample has uniform intensity, making the evaluation of the susceptibility easier.

For a small sample, the static and the microwave magnetic fields may be expressed in the most general form as (using Equation 20 and Equation 21)

$$
\mathbf{H}_{\mathrm{i}}=\mathbf{H}_{\mathbf{0}}-\mathbf{N} \cdot \mathbf{M}_{\mathrm{s}} \text { and } \mathbf{H}_{\mathrm{ac}, \mathrm{i}}=\mathbf{H}_{\mathrm{ac}}-\mathbf{N} \cdot \mathbf{M}_{\mathrm{ac}}
$$

\section{Equation 64}

where $\mathbf{N}$ is the demagnetization vector that is a measure of the opposing internal field due to the presence of dipoles induced on the surface. Assuming that both $\mathbf{H}_{\mathbf{0}}$ and $\mathbf{M}_{\mathrm{s}}$ are in the $z$-direction and $\mathbf{H}_{\mathbf{a c}}$ is applied in the $x-y$ plane, the complex susceptibility tensor components including the loss terms can be obtained by substituting Equation 64 into the equation of motion (Equation 19) in phasor form (assuming $e^{j \omega t}$ dependence) as

$$
\chi_{x x, d}=\frac{\omega_{m}\left[\left(\omega_{0, d}+j \phi \omega\right)+N_{y} \omega_{m}\right]}{\omega_{r}^{2}-\omega^{2}+2 j \phi \omega\left[\omega_{0, d}+\omega_{m}\left(N_{x}-N_{y}\right) / 2\right]}
$$

\section{Equation 65}

$$
\chi_{y y, d}=\frac{\omega_{m}\left[\left(\omega_{0, d}+j \phi \omega\right)+N_{x} \omega_{m}\right]}{\omega_{r}^{2}-\omega^{2}+2 j \phi \omega\left[\omega_{0, d}+\omega_{m}\left(N_{x}-N_{y}\right) / 2\right]}
$$

Equation 66

$$
\chi_{y x, d}=-\chi_{x y, d}=\frac{j \omega \omega_{m}}{\omega_{r}^{2}-\omega^{2}+2 j \phi \omega\left[\omega_{0, d}+\omega_{m}\left(N_{x}-N_{y}\right) / 2\right]}
$$

\section{Equation 67}


where

$$
\omega_{0, d}=\gamma \mu_{0} H_{0}-N_{z} \omega_{m} \text { and } \omega_{r}=\left\{\left[\gamma \mu_{0} H_{0}+\left(N_{x}-N_{z}\right) \omega_{m}\right]\left[\gamma \mu_{0} H_{0}+\left(N_{y}-N_{z}\right) \omega_{m}\right]\right\}^{1 / 2}
$$

\section{Equation 68}

and $\omega_{m}$ is defined in Equation 27.

It is clear from Equation 65, Equation 66, and Equation 67 that the resonance condition has been altered by the introduction of the demagnetization factors. Note that damping has been introduced to the numerators of the susceptibility expressions by adding $j \phi \omega$ to $\omega_{0, d}$. However, this does not appear explicitly in the denominators, impeding the analysis of microwave ferrite devices. Therefore, an approximation that would allow rapid practical applications of these susceptibility formulas is often adopted. ${ }^{32}$ In this approximation, the denominators in Equation 65, Equation 66, and Equation 67 are replaced with $\left(\omega_{r}+j \phi \omega\right)^{2}-\omega^{2}$, where the loss term $(j \phi \omega)$ is added to $\omega_{r}$, as done for $\omega_{0}$ in the numerators. The expressions are still accurate near the resonance for typical microwave device shapes and no information is lost at the expense of simplifying the analysis. The real and imaginary parts of the susceptibility components described in Equation 40 and Equation 41 can now be written with this approximation as

$$
\chi_{x x, d}^{\prime}=\frac{\omega_{m}\left[\omega_{r}^{2}-\omega^{2}\left(1+\phi^{2}\right)\right]\left(\omega_{0}+N_{y} \omega_{m}\right)+2 \omega_{r} \phi^{2} \omega^{2}}{\left(\omega_{r}^{2}-\omega^{2}-\phi^{2} \omega^{2}\right)^{2}+4 \phi^{2} \omega^{2} \omega_{r}^{2}}
$$

\section{Equation 69}


Özgür et al., submitted to Journal of Materials Science: Materials in Electronics, 2009 Microwave Ferrites, Part 1: Fundamental properties

$$
\chi_{x x, d}^{\prime \prime}=\frac{2 \omega_{m} \omega_{r} \phi \omega\left(\omega_{0}+N_{y} \omega_{m}-\phi \omega\right)}{\left(\omega_{r}^{2}-\omega^{2}-\phi^{2} \omega^{2}\right)^{2}+4 \phi^{2} \omega^{2} \omega_{r}^{2}}
$$

Equation 70

$$
\chi_{x y, d}^{\prime}=\frac{\omega \omega_{m}\left[\omega_{r}^{2}-\omega^{2}\left(1+\phi^{2}\right)\right]}{\left(\omega_{r}^{2}-\omega^{2}-\phi^{2} \omega^{2}\right)^{2}+4 \phi^{2} \omega^{2} \omega_{r}^{2}}
$$

Equation 71

$$
\chi_{x y, d}^{\prime \prime}=\frac{2 \omega_{m} \omega_{r} \phi \omega^{2}}{\left(\omega_{r}^{2}-\omega^{2}-\phi^{2} \omega^{2}\right)^{2}+4 \phi^{2} \omega^{2} \omega_{r}^{2}}
$$

\section{Equation 72}

The components $\chi_{y y, d}$ can be obtained by substituting $N_{x}$ for $N_{y}$ in the expressions for $\chi_{x x, d}$.

For the operation of microwave devices the loss term should be small enough, i.e. we need to have $\omega_{r} \gg \phi \omega$. This validates the approximation involved in the phenomenological representation of loss in Equation 65, Equation 66, and Equation 67. These simpler expressions are very convenient in the treatment of microwave devices with demagnetization factors.

The solution of the electromagnetic boundary-value problem needed to evaluate the magnetic fields inside a ferrite is very difficult for an arbitrary sample shape. Additionally, Equation 68 fundamentally applies to samples that are very small compared to the wavelength or the skin depth. However, the resonance condition can still be 
Özgür et al., submitted to Journal of Materials Science: Materials in Electronics, 2009 Microwave Ferrites, Part 1: Fundamental properties

approximately predicted for larger specimens used in ferrite devices if they have typical geometrical shapes. For example, for a thin uniaxial slab with the z-axis perpendicular to the surface, the demagnetization factors are $N_{x}=N_{y}=0$ and $N_{z}=1 .^{32}$ These factors should always satisfy $N_{x}+N_{z}+N_{y}=1$. If the DC magnetic field is applied along the zdirection (perpendicular to the sample plane), from Equation 68 we obtain for a uniaxial slab (in cgs units)

$$
\begin{aligned}
& \omega_{r}=\omega_{0}-\omega_{m}=\gamma\left(H_{0}-4 \pi M_{s}\right)=\frac{g \mu_{B}}{\hbar}\left(H_{0}-4 \pi M_{s}\right) \\
& \text { or } \quad f_{r} \cong(2.8 \mathrm{MHz} / \mathrm{Oe})\left(H_{0}-4 \pi M_{s}\right) \quad\left(\text { slab }, \mathrm{H}_{0} \perp \text { surface }\right)
\end{aligned}
$$

\section{Equation $\mathbf{7 3}$}

It should be noted that the factor $2.8 \mathrm{MHz} / \mathrm{Oe}$ is obtained by assuming $g=2$. In fact, the Landé g-factor and therefore a more accurate value of this factor $(2.8 \mathrm{MHz} / \mathrm{Oe})$ can be obtained from the measurements of the FMR frequency as a function of the applied field using Equation 73. However, for simplicity and practical purposes, the $2.8 \mathrm{MHz} / \mathrm{Oe}$ factor will be used from here on. Notice that in order to achieve magnetic saturation and to observe resonance, one should have $H_{0}>4 \pi M_{s}$. If the DC magnetic field is applied parallel to the sample plane, choosing again the z-direction to be the direction of the applied magnetic field (and assuming surface normal is along the $y$-axis), the demagnetization factors become $N_{x}=N_{z}=0$ and $N_{y}=1 .{ }^{32}$ Equation 68 will then have the form (in cgs units)

$$
\begin{aligned}
& \omega_{r}=\left\{\omega_{0}\left[\omega_{0}+\omega_{m}\right]\right\}^{1 / 2}=\left\{\gamma H_{0}\left[\gamma\left(H_{0}+4 \pi M_{s}\right)\right]\right\}^{1 / 2} \\
& \text { or } \quad f_{r} \cong(2.8 \mathrm{MHz} / \mathrm{Oe})\left[H_{0}\left(H_{0}+4 \pi M_{s}\right)\right]^{1 / 2} \quad \text { (slab, } \mathrm{H}_{0} / / \text { surface) }
\end{aligned}
$$


Özgür et al., submitted to Journal of Materials Science: Materials in Electronics, 2009 Microwave Ferrites, Part 1: Fundamental properties

\section{Equation 74}

The demagnetization factors for a cylindrical rod with the axis of symmetry along the zaxis are $N_{x}=N_{y}=1 / 2$ and $N_{z}=0$. If the magnetic field is applied along the z-axis the resonance frequency for an infinitely long cylindrical rod is

$$
\begin{aligned}
& \omega_{r}=\gamma\left(H_{0}+2 \pi M_{s}\right) \\
& \text { or } \quad f_{r} \cong(2.8 \mathrm{MHz} / \mathrm{Oe})\left(H_{0}+2 \pi M_{s}\right) \quad \text { (cylindrical rod) }
\end{aligned}
$$

\section{Equation 75}

For a spherical sample $N_{x}=N_{z}=N_{y}=1 / 3$. Therefore, the expression for the resonance frequency for a spherical specimen is

$$
\omega_{r}=\omega_{0}=\gamma H_{0} \quad \text { or } \quad f_{r} \cong(2.8 \mathrm{MHz} / \mathrm{Oe}) H_{0} \quad \text { (sphere) }
$$

\section{Equation 76}

which is the same as that for an infinite medium. This is the reason why small spherical ferrite samples are used in microwave cavity experiments to measure the permeability. Spherical shaped ferrites are also preferred for magnetization anisotrophy measurements as the internal demagnetization fields cancel out for spherical symmetry.

\subsection{Anisotropy}

In addition to the geometrical effects, internal fields may also arise from the magnetic anisotropy in a sample. Depending on the magnetization direction relative to the principal 
Özgür et al., submitted to Journal of Materials Science: Materials in Electronics, 2009 Microwave Ferrites, Part 1: Fundamental properties

axes of the crystal, an additional energy, anisotropy energy, may be present in ferromagnetic crystals. In a single crystal, the additional torque exerted on the magnetization vector due to the effective anisotropy field $\mathbf{H}_{\mathbf{a}}$ is (similar to Equation 18)

$$
\mathbf{T}_{\mathbf{a}}=\mu_{0} \mathbf{M} \times \mathbf{H}_{\mathbf{a}}
$$

\section{Equation 77}

The magnitude of the torque is given by $T_{a}=d \varepsilon_{a} / d \theta$, where $\varepsilon_{a}$ is the anisotropy energy and $\theta$ is the angle of rotation about the axis perpendicular to $\mathbf{M} \times \mathbf{H}_{\mathbf{a}} \cdot \mathbf{H}_{\mathbf{a}}$ may be chosen to lie along either the $x$ - or the $y$-axis and we can write its components in terms of an effective demagnetization factor as $H_{x}^{a}=-N_{x}^{a} M_{x}$ and $H_{y}^{a}=-N_{y}^{a} M_{y}$ (Similarly, if present, the $z$ component may also be expressed as $\left.H_{z}^{a}=-N_{z}^{a} M_{z}\right) \cdot{ }^{40}$ We can then simply add the magnetic anisotropy demagnetization factors to the geometrical demagnetization factors to obtain the new form of the resonance condition defined in Equation 68 as

$$
\omega_{r}=\left\{\left[\mu_{0} \gamma H_{0}+\left(N_{x}+N_{x}^{a}-N_{z}\right) \omega_{m}\right]\left[\mu_{0} \gamma H_{0}+\left(N_{y}+N_{y}^{a}-N_{z}\right) \omega_{m}\right]\right\}^{1 / 2}
$$

\section{Equation 78}

This is the general resonance condition where the externally applied field and one of the principal axes of the single crystal are chosen to both lie along the $z$-direction. It is evident that the resonance frequency is shifted to higher frequencies by the anisotropy.

For cubic crystals if the external DC field is applied along the [001] axis, which is chosen as the $z$-axis, and the RF field along the [100] direction ( $x$-axis), M will lie in the $y$ - $z$ plane and will be off from the z-axis by a small angle $\theta$. This would lead 
Özgür et al., submitted to Journal of Materials Science: Materials in Electronics, 2009 Microwave Ferrites, Part 1: Fundamental properties

to $\varepsilon_{a}=K_{1} \sin ^{2} \theta \cos ^{2} \theta,{ }^{32,40}$ where $K_{1}$ is the first order anisotropy constant. By using $H_{x}^{a}=-N_{x}^{a} M_{x}$ one can then obtain the torque as

$$
T_{a}=d \varepsilon_{a} / d \theta=K_{1} \sin 2 \theta \cos 2 \theta=\left|\mathbf{M} \times \mathbf{H}_{\mathbf{a}}\right| \approx M_{s} N_{x}^{a} M_{x}
$$

\section{Equation 79}

Here, we have assumed that the static magnetic field is sufficiently large so that the total magnetization mostly lies along the z-axis since $\theta$ is very small, and $M_{z} \approx M_{s}$. Similarly, for a small value of $\theta, M_{x} \approx M_{z} \sin \theta \approx M_{s} \sin \theta$. This would lead to

$$
K_{1} \sin 2 \theta \cos 2 \theta \approx N_{x}^{a} M_{s}^{2} \sin \theta
$$

\section{Equation 80}

so that for angles near $\theta=0$

$$
N_{x}^{a}=N_{y}^{a}=\frac{2 K_{1}}{M_{s}^{2}}
$$

\section{Equation 81}

Similarly, for uniaxial crystals, $\varepsilon_{a}=K_{1} \sin ^{2} \theta$. If the external DC field is applied along the uniaxial direction that is chosen to lie along the $z$-axis, and the RF field is applied along the $x$-direction, the magnetization vector will again make a very small angle, $\theta$, with the z-axis. Using the same approach as in the case of cubic crystals, this will lead to $T_{a}=d \varepsilon_{a} / d \theta=K_{1} \sin 2 \theta \approx N_{x}^{a} M_{s}^{2} \sin \theta$, and for small $\theta$ we obtain the same relationship in Equation 81, form which it is evident that the effective anisotropy field is

$$
H_{a}=\frac{2 K_{1}}{M_{s}}
$$


Özgür et al., submitted to Journal of Materials Science: Materials in Electronics, 2009 Microwave Ferrites, Part 1: Fundamental properties

\section{Equation 82}

In order to estimate the resonance frequencies in the presence of an anisotropy field, $H_{a}$ should be added to the applied field $H_{0}$ in the expressions given in Equation 73 , Equation 74, Equation 75, and Equation 76 for different ferrite shapes. For example, a ferrite specimen in the form of a uniaxial slab under a DC magnetic field applied perpendicular to the slab surface will have a resonance frequency given by

$$
\left.f_{r} \cong(2.8 \mathrm{MHz} / \mathrm{Oe})\left(H_{0}+H_{a}-4 \pi M_{s}\right) \quad \quad \text { (slab, } \mathrm{H}_{0} \perp \text { surface }\right)
$$

\section{Equation 83}

Similarly, the resonance frequency for a spherical ferrite specimen is given by

$$
f_{r} \cong(2.8 \mathrm{MHz} / \mathrm{Oe})\left(H_{0}+H_{a}\right) \quad \text { (sphere) }
$$

\section{Equation 84}

Equation 84 and Equation 83 indicate that the ferromagnetic (or ferrimagnetic) resonance can be observed in ferrites having nonzero anisotropy even without any external magnetic field present. This leads to possibility of self biasing, which will be discussed in Section 5.4 .

The effective anisotropy field actually depends upon the angle that the applied magnetic field makes with the single crystal axes. Consequently, the resonance condition depends on the crystal orientation and for a fixed frequency, this requires adjustments on the applied DC field intensity to achieve resonance. For instance, the magnetic field required for resonance in (100) plane cubic crystal is expected to be greater when the external DC field is applied along the [110] direction than when it is applied along the [100] 
Özgür et al., submitted to Journal of Materials Science: Materials in Electronics, 2009 Microwave Ferrites, Part 1: Fundamental properties

direction. ${ }^{40}$ Anisotropy field and saturation magnetization values for various materials are listed in Table $6 .^{32,41,42}$

\subsection{Crystalline imperfections / polycrystalline ferrites}

We have so far discussed anisotropy in the context of single crystal structures, for which the shift in the resonance condition depends on the orientation of the applied field with respect to the principal crystal axes. In a polycrystalline sample consisting of randomly oriented crystallites, this orientation varies through all possibilities. Consequently, the resonance absorption is broadened. In addition, demagnetization effects due to grain boundaries, voids, or inhomogeneous regions also contribute to this broadening as the resonance frequency in each of these regions is shifted by a different amount. In a crystal with imperfections an additional DC field exists in the vicinity of the pores and other nonmagnetic inclusions as there will be magnetic poles induced at the surfaces of all these inhomogeneities. For instance, the additional DC field due to a spherical pore at the center of a spherical sample is given by ${ }^{43}$

$$
H_{i}^{p}=\frac{4 \pi M_{s}}{3} \frac{v}{V}
$$

\section{Equation 85}

where $v / V$ is the ratio of the pore volume to the ferrite volume. The resonance is shifted by an amount $\gamma \mu_{0} H_{i}^{p}$ and the linewidth is broadened by

$$
\Delta H^{p}=6 \pi M_{s} \frac{v}{V}
$$

\section{Equation 86}


In polycrystalline ferrites, random anisotropy field and residual porosity may contribute to the linewidth broadening, and the line shape is not Lorentzian due to spin-wave excitation. $^{44}$

\subsection{Ferrimagnetic resonance}

In the preceding Sections $(4.1-4.5)$, we have focused on ferromagnetic materials. For microwave applications, ferrimagnetic materials, which are special cases of antiferromagnetic materials as discussed before, are of special interest. The structure of ferrimagnetic materials though can be very complex as they may contain multiple sublattices with different magnetic moments, which may be oriented differently in two or even three dimensions. However, for simplicity, we shall discuss here magnetic systems with two-sublattices that are oriented antiparallel to each other as discussed in Section 2.1.

The total magnetic moment differs from that due to spin alone due to the spin-orbit coupling. Since both the orbital motion and the spin-orbit coupling depend upon the crystal structure, in ferrites that have more than one type of crystallographic site, i.e. sublattice, the gyromagnetic ratio, the total magnetic moment of an ion, and the anisotropy field may be different in one sublattice than in the other. The total magnetization of a ferromagnetic system may be expressed as

$$
\mathbf{M}=\sum \mathbf{M}_{i}=\sum \gamma_{i} \mathbf{S}_{i}
$$

\section{Equation 87}


Özgür et al., submitted to Journal of Materials Science: Materials in Electronics, 2009 Microwave Ferrites, Part 1: Fundamental properties

where $\mathbf{M}_{i}$ is the magnetization, $\gamma_{i}$ is the gyromagnetic ratio, and $\mathbf{S}_{i}$ is the spin angular momentum of the $i$ th lattice. The equation of motion for the magnetization in the $i$ th sublattice without the losses would therefore be

$$
\frac{d \mathbf{M}_{i}}{d t}=\gamma_{i} \mu_{0} \mathbf{M}_{i} \times \mathbf{H}_{i}
$$

\section{Equation 88}

where $\mathbf{H}_{i}$ represents the total magnetic field acting on the magnetic ions in the $i$ th sublattice and includes the applied field $\mathbf{H}_{\mathbf{0}}$, the exchange field, the effective magnetic anisotrophy field, and the demagnetization fields associated with the geometrical shape of the sample. Neglecting the anisotropy and the demagnetization fields the total magnetic field can be expressed as

$$
\mathbf{H}_{i}=\mathbf{H}_{\mathbf{0}}-\sum_{i \neq j} \lambda_{E, i j} \mathbf{M}_{j}
$$

\section{Equation 89}

where $\lambda_{E, i j} \mathbf{M}_{j}$ is the exchange field (see Equation 14) of the dipoles in the $j$ th sublattice acting on the dipoles in the $i$ th sublattice.

Now let's look into a two-sublattice system and assume that the time dependent components of the magnetic field and the magnetization go as $e^{j \omega t}$ and that the anisotropy fields acting on the two sublattices are oppositely oriented along a preferred axis of the crystal. We can denote the anisotropy fields as $+H_{a 1}$ and $-H_{a 2}$, the saturation magnetization vectors as $\mathbf{M}_{1}=M_{1} \hat{\mathbf{z}}$ and $\mathbf{M}_{2}=-M_{2} \hat{\mathbf{z}}$, and the exchange fields as $+\lambda_{E} M_{2}$ and $-\lambda_{E} M_{1}$, for the sublattices 1 and 2, respectively, where $M_{1}$ and $M_{2}$ have been chosen 
Özgür et al., submitted to Journal of Materials Science: Materials in Electronics, 2009 Microwave Ferrites, Part 1: Fundamental properties

as positive quantities. The exchange field coefficient is denoted by $\lambda_{E}$ with no indices as $\lambda_{E, 12}=\lambda_{E, 21}$. It is convenient to assume that the applied field $\mathbf{H}_{\mathbf{0}}$ is along the $z$ direction, the RF field is in the $x-y$ plane, $M_{2}>M_{1}>>H_{\mathrm{a}}, H_{0}$, and the total magnetic field and the total magnetization vectors with both the $\mathrm{DC}$ and $\mathrm{AC}$ components are of the form

$$
\begin{aligned}
& \mathbf{H}_{1}=\hat{\mathbf{z}}\left(H_{0}+H_{a 1}+\lambda_{E} M_{2}\right)+\mathbf{H}_{\mathbf{a c}} ; \mathbf{H}_{2}=\hat{\mathbf{z}}\left(H_{0}-H_{a 2}-\lambda_{E} M_{1}\right)+\mathbf{H}_{\mathbf{a c}} \\
& \mathbf{M}_{1}^{\prime}=\hat{\mathbf{z}} M_{1}+\mathbf{M}_{\mathbf{a c}, 1} ; \mathbf{M}_{2}^{\prime}=\hat{\mathbf{z}} M_{2}+\mathbf{M}_{\mathbf{a c}, 2}
\end{aligned}
$$

\section{Equation 90}

where the $\mathrm{AC}$ components have the same $e^{j \omega t}$ time dependence. The equation of motion can be solved in a rotating frame of reference, where the AC components of the magnetic field and the magnetization can be represented by

$$
H_{a c}^{ \pm}=H_{x} \pm j H_{y} \quad \text { and } \quad M_{a c_{1,2}}^{ \pm}=M_{x_{1,2}} \pm j M_{y_{1,2}}
$$

\section{Equation 91}

Solving for $M_{a c_{1}}^{ \pm}$and $M_{a c_{2}}^{ \pm}$yields

$$
\begin{aligned}
& M_{a c_{1}}^{ \pm}=\frac{\left(\omega_{2} \pm \omega+\lambda_{E} \mu_{0} \gamma_{2} M_{2}\right) \mu_{0} \gamma_{1} M_{1} H_{a c}^{ \pm}}{\left(\omega \pm \omega_{1}\right)\left(\omega \pm \omega_{2}\right)+\lambda_{E}^{2} \mu_{0}^{2} \gamma_{1} \gamma_{2} M_{1} M_{2}} \\
& M_{a c_{2}}^{ \pm}=\frac{-\left(\omega_{1} \pm \omega-\lambda_{E} \mu_{0} \gamma_{1} M_{1}\right) \mu_{0} \gamma_{2} M_{2} H_{a c}^{ \pm}}{\left(\omega \pm \omega_{1}\right)\left(\omega \pm \omega_{2}\right)+\lambda_{E}^{2} \mu_{0}^{2} \gamma_{1} \gamma_{2} M_{1} M_{2}}
\end{aligned}
$$

\section{Equation 92}

and for the resonance frequencies we obtain the quadratic equation

$$
\omega^{2} \pm\left(\omega_{1}+\omega_{2}\right) \omega+\omega_{1} \omega_{2}+\lambda_{E}^{2} \mu_{0}^{2} \gamma_{1} \gamma_{2} M_{1} M_{2}=0
$$

\section{Equation 93}


Özgür et al., submitted to Journal of Materials Science: Materials in Electronics, 2009 Microwave Ferrites, Part 1: Fundamental properties

where

$$
\omega_{1}=\gamma_{1} \mu_{0}\left(H_{0}+H_{a 1}+\lambda_{E} M_{2}\right) \text { and } \quad \omega_{2}=\gamma_{2} \mu_{0}\left(H_{0}-H_{a 2}-\lambda_{E} M_{1}\right)
$$

\section{Equation 94}

The resonance frequencies are therefore given by

$$
\omega_{ \pm}=\frac{\mp\left(\omega_{1}+\omega_{2}\right) \pm \sqrt{\left(\omega_{1}-\omega_{2}\right)^{2}-4 \lambda_{E}^{2} \mu_{0}^{2} \gamma_{1} \gamma_{2} M_{1} M_{2}}}{2}
$$

\section{Equation 95}

It is clear that one of the frequencies is negative as the term in the square root is larger than $\left(\omega_{1}+\omega_{2}\right)$.

The magnetic susceptibilities can be easily derived for the two rotating modes from

$$
\chi_{ \pm}=\frac{M_{a c, 1}^{ \pm}+M_{a c, 2}^{ \pm}}{H_{a c}^{ \pm}}
$$

\section{Equation 96}

Using Equation 92 and Equation 94 we can obtain the total susceptibility as $\chi_{ \pm}=\frac{\gamma_{1} \gamma_{2} \mu_{0}^{2}\left(M_{1}-M_{2}\right) H_{0}-\gamma_{1} \gamma_{2} \mu_{0}^{2}\left(H_{a 1} M_{2}+H_{a 2} M_{1}\right) \pm \mu_{0}\left(\gamma_{1} M_{1}-\gamma_{2} M_{2}\right) \omega-\lambda_{E} \mu_{0}^{2} \gamma_{1} \gamma_{2}\left(M_{1}-M_{2}\right)^{2}}{\left[\omega \pm \gamma_{1} \mu_{0}\left(H_{0}+H_{a 1}+\lambda_{E} M_{2}\right)\right]\left[\omega \pm \gamma_{2} \mu_{0}\left(H_{0}-H_{a 2}-\lambda_{E} M_{1}\right)\right]+\lambda_{E}^{2} \mu_{0}^{2} \gamma_{1} \gamma_{2} M_{1} M_{2}}$

\section{Equation 97}

The ferromagnetic resonance occurs in the microwave frequency range when a DC magnetic field of a few thousand Oe is applied. The ferromagnetic resonance mode in a two-sublattice system corresponds to two sets of spins precessing in phase in the same rotational direction. However, for the two sets of spins precessing in the same rotational 
Özgür et al., submitted to Journal of Materials Science: Materials in Electronics, 2009 Microwave Ferrites, Part 1: Fundamental properties

direction but out of phase, there exists another resonance at a much higher frequency, called the exchange resonance since it takes place in the exchange field, which is considerably larger than any externally applied magnetic field. Simple expressions for these two resonance frequencies may be obtained from Equation 93. In the low frequency limit, i.e. ferromagnetic mode, the $\omega^{2}$ term and the factors that are small compared to the exchange fields $\lambda_{E} M_{1}$ and $\lambda_{E} M_{2}$ can be neglected giving

$$
\omega_{0, L F} \approx \frac{\mu_{0}\left(M_{1}-M_{2}\right)}{M_{1} / \gamma_{1}-M_{2} / \gamma_{2}}\left(H_{0}+\frac{H_{a 1} M_{1}+H_{a 2} M_{2}}{M_{1}-M_{2}}\right)
$$

\section{Equation 98}

The high resonance frequency, i.e. exchange resonance, can be obtained by assuming that $\omega$ is very large, and therefore, the constant term in Equation 93 can be neglected. This would give

$$
\omega_{0, H F} \approx \lambda_{E} \mu_{0}\left(\gamma_{2} M_{1}-\gamma_{1} M_{2}\right)
$$

\section{Equation 99}

If we do a second iteration using the solution of Equation 99 and keeping the constant term in Equation 93 we obtain

$$
\omega_{0, H F} \approx \lambda_{E} \mu_{0}\left(\gamma_{2} M_{1}-\gamma_{1} M_{2}\right)+\frac{\mu_{0}\left[\left(\gamma_{1}^{2} M_{2}-\gamma_{2}^{2} M_{1}\right) H_{0}+\gamma_{1}^{2} M_{2} H_{a 1}+\gamma_{2}^{2} M_{1} H_{a 2}\right]}{\gamma_{2} M_{1}-\gamma_{1} M_{2}}
$$

\section{Equation 100}

Usually, the exchange resonance for ferrites occurs in the far-infrared wavelength range. However, near a compensation point, i.e. when $\gamma_{1} M_{2}=\gamma_{2} M_{1}$, for anisotropy fields in the 
Özgür et al., submitted to Journal of Materials Science: Materials in Electronics, 2009 Microwave Ferrites, Part 1: Fundamental properties

order of several hundred Gauss, the exchange resonance can be observed at high microwave frequencies.

The loss can also be introduced into the ferrimagnetic systems as described previously for the ferromagnetic systems (see Equation 38). The equation of motion for each sublattice including damping can be written as

$$
\frac{d \mathbf{M}_{i}}{d t}=\mu_{0} \gamma_{i} \mathbf{M}_{i} \times \mathbf{H}_{i}-\frac{\mu_{0} \phi_{i} \gamma_{i} \mathbf{M}_{i}}{M_{i}} \times\left(\mathbf{M}_{i} \times \mathbf{H}_{i}\right)
$$

\section{Equation 101}

where $\phi_{i}$ is the damping parameter for the $i$ th sublattice. Assuming the loss is sufficiently small to allow neglecting the terms in second and higher orders of $\phi$, the equation of motion can be solved to obtain the susceptibility components using the same mathematical techniques described in Sections 4.2 and 4.3.

\subsection{Loss Mechanisms in Ferrites}

For microwave devices, the insertion loss, which includes magnetic, dielectric, and conductor losses associated with all components, is an extremely important parameter for evaluation of performance. The off-resonance loss in ferrites in devices such as circulators and microstrip tunable filters (see Section 3 in Part 2 for various device applications) $)^{3}$ is of significant importance. 
Özgür et al., submitted to Journal of Materials Science: Materials in Electronics, 2009 Microwave Ferrites, Part 1: Fundamental properties

The dielectric loss due to damping of the vibrating electric dipole moments arises from the imaginary part of the permittivity, $\varepsilon=\varepsilon^{\prime}-j \varepsilon^{\prime \prime}$. In a linear medium with conductivity $\sigma$ the conduction current density is given as $\mathbf{J}=\sigma \mathbf{E}$ and the electric displacement as $\mathbf{D}=\varepsilon \mathbf{E}$ in terms of the electric field $\mathbf{E}$. Maxwell's curl equation for $\mathbf{H}$ can then be written for plane waves as

$$
\begin{aligned}
\nabla \times \mathbf{H} & =\frac{\partial \mathbf{D}}{\partial t}+\mathbf{J}=j \omega \mathbf{D}+\sigma \mathbf{E} \\
& =j \omega \varepsilon \mathbf{E}+\sigma \mathbf{E} \\
& =j \omega\left[\varepsilon^{\prime}-j\left(\varepsilon^{\prime \prime}+\frac{\sigma}{\omega}\right)\right] \mathbf{E}
\end{aligned}
$$

\section{Equation 102}

It is clear from Equation 102 that the dielectric and conduction losses are indistinguishable. When defining losses in a medium, loss tangent is the commonly used parameter, which is defined by

$$
\tan \delta=\frac{\omega \varepsilon^{\prime \prime}+\sigma}{\omega \varepsilon^{\prime}}
$$

\section{Equation 103}

In a nonconducting or poorly conducting medium, the loss tangent is given simply by the ratio of the imaginary and real parts of the permittivity. Equations derived for the lossless media can easily transformed to include the dielectric loss by replacing the real permittivity with $\varepsilon=\varepsilon_{0} \varepsilon_{r}(1-j \tan \delta)$, where $\varepsilon_{r}=\varepsilon^{\prime} / \varepsilon_{0}$ is the relative dielectric constant.

Dielectric loss tangent and dielectric constant for some ferrite materials manufactured by Trans-Tech, Inc. ${ }^{\text {iii }}$ are listed in Table 7.

\footnotetext{
iii http://www.trans-techinc.com
} 
Apart from losses due to dielectric dissipation and imperfect conductors, the total loss in microwave devices is mainly determined by the magnetic damping associated with the ferromagnetic resonance. ${ }^{36}$ Therefore, development of single crystal ferrite materials with narrow FMR linewidths, indicating low magnetic loss, has been one of the major goals. Following the description of Schloemann, ${ }^{45}$ Figure 15 shows the magnetic relaxation processes that affect the FMR damping in ferrites. A group of spin waves that is excited by the electromagnetic signal can distribute its energy either within the magnetic system, i.e. system of electron spins responsible for the ferromagnetism that are coupled by the exchange and dipolar interactions, or through interaction with charge carriers, lattice vibrations, and strongly relaxing ions. In addition to the purely intrinsic losses related to fundamental interactions in ferromagnetic materials within the magnetic system extrinsic losses may also arise from the crystal imperfections (dependent on the growth method and conditions), polycystallinity (random local anisotropy), porosity, grain boundaries, surface roughness, or fast and slow relaxing impurities $\left(\mathrm{Fe}^{+3}, \mathrm{Fe}^{2+}\right.$ hopping, rare earth slow relaxers, etc.).

In most ferrites, the extrinsic processes are the ones that mainly determine the FMR linewidth, and the intrinsic processes determine the threshold for high power spin wave instability. Especially in polycrystalline ferrites intrinsic processes are negligible compared to the effects due to residual porosity and random anisotropy. Moreover, the FMR lineshape resulting from extrinsic processes is usually non-Lorentzian. It should be noted that the FMR linewidth, however, is not a good indicator for loss in devices that 
Özgür et al., submitted to Journal of Materials Science: Materials in Electronics, 2009 Microwave Ferrites, Part 1: Fundamental properties

operate off resonance under a relatively small bias magnetic field, such as circulators, tunable filters, and phase shifters. Therefore, the off-resonance linewidth, also referred to as the effective linewidth, is used to characterize the magnetic loss in such devices. ${ }^{46}$

The spin relaxation processes are usually described by the interaction of magnons, which are elementary excitations in a magnetic system. The spin relaxation processes are classified as two, three, and four magnon processes, as they are represented by the terms in the spin-wave Hamiltonian that are of second, third, and fourth order, respectively, in the spin-wave amplitudes. ${ }^{47,48}$ The two-magnon scattering, which is extrinsic, arise from the inhomogeneities in the material, such as crystal imperfections, randomly oriented crystallites, porosity, surface roughness, and impurities. This is the dominant loss mechanism in coarse-grain sintered polycrystalline ferrites with a small porosity and low magnetocrystalline anisotropy. The three-magnon processes, induced by dipolar interaction, and the four-magnon processes, induced by both dipolar and exchange interactions, are truly intrinsic. The three-magnon processes, where a directly excited magnon splits into two magnons under conservation of energy and momentum, contribute to the off-resonance damping under the condition $H_{i} \leq \omega /\left(2 \gamma \mu_{0}\right)$, assuming that there is no anisotropy field, where $H_{i}$ is the internal magnetic field, and $\omega$ is the signal frequency. A detailed discussion of these relaxation processes can be found in Ref. 48 .

The relaxation processes mentioned above are usually represented by three linewidths in magnetic materials: the FMR linewidth (half power linewidth in field), the fielddependent effective linewidth, and the spin-wave linewidth. All these linewidths are 
Özgür et al., submitted to Journal of Materials Science: Materials in Electronics, 2009 Microwave Ferrites, Part 1: Fundamental properties

generally much larger in polycrystalline materials than in single crystal varieties. The FMR linewidth is influenced mainly by the extrinsic two-magnon scattering processes, and as indicated can be large in polycrystalline materials. Typical FMR linewidths in single crystals are on the order or $1 \mathrm{Oe}$, while that in polycrystalline ferrites can be from tens to hundreds of Oe (See Table 7). The effective linewidth, which is a measure of the off-resonance loss describing the relaxation of uniform precession of fields away from the resonance, is generally much smaller than the FMR linewidth and is not affected by the two-magnon processes. ${ }^{46}$ The spin wave linewidth represents the relaxation rate of parametric spin waves generated through nonlinear processes and can be very small compared to the other two linewidths. The spin-wave linewidth is obtained from a theoretical analysis using the measured spin-wave instability threshold field. ${ }^{49}$ The effective linewidth is derived from the complex susceptibility obtained from measurements of the cavity $\mathrm{Q}$ factor and resonance frequency measurements as a function of the applied field. ${ }^{46}$

Using the phenomenological equation of motion for the magnetization from Gilbert (Equation 39), ${ }^{39}$ one can obtain the effective susceptibility as ${ }^{50}$

$$
\chi_{e}=\left(\chi_{e}^{\prime}-j \chi_{e}^{\prime \prime}\right)=\frac{\gamma M\left(\gamma H_{0}+j \eta\right)}{\left(\gamma H_{0}+j \eta\right)^{2}-\omega^{2}}
$$

\section{Equation 104}

where $\eta$ is the relaxation rate. If $\chi^{\prime}$ and $\chi_{e}^{\prime \prime}$ are measured, $\eta$ and $\gamma$ can be easily obtained by replacing the gyromagnetic ratio in the numerator with that of the free electron. If $\eta$ and $\gamma$ are allowed to be field dependent, the effective linewidth is defined as 
Özgür et al., submitted to Journal of Materials Science: Materials in Electronics, 2009 Microwave Ferrites, Part 1: Fundamental properties

$$
\Delta H_{e f f}=2 \eta\left(H_{0}\right) / \gamma\left(H_{0}\right)
$$

\section{Equation 105}

Representative FMR linewidth data measured for hot isostatic pressed (hipped) polycrystalline YIG is shown in Figure $16 .{ }^{49}$ The data matches with that expected from two-magnon processes due to magnetocrystalline anisotrophy in the randomly oriented grains. The linewidth measured at $9.5 \mathrm{GHz}$ was 13 Oe for a spherical sample. The closeness of the material density to its theoretical value is supported by the fact that porosity would contribute approximately 23 Oe to the FMR linewidth at $10 \mathrm{GHz}$ per percent porosity and would broaden the linewidth further with increasing frequency, ${ }^{51}$ which is not the case as shown in Figure 16. The effective linewidth measurements on the same sample revealed low and high field values of 3 and 1.7 Oe (between 5 and 8 kOe of external field, $10 \mathrm{GHz}$ ), respectively. Furthermore, the spin wave linewidth was determined to be 0.45 Oe at $8.35 \mathrm{GHz}$. High field effective linewidth measurements on conventionally sintered and hipped YIG samples indicate that even the half power FMR linewidths are significantly different (30 and $13.5 \mathrm{Oe}$, respectively at $9.5 \mathrm{GHz}$ ), the effective linewidths at extreme high field limit ( $>5 \mathrm{kOe}$ at $10 \mathrm{GHz}$ ) approach to the intrinsic single crsytal linewidths $(<1 \mathrm{Oe})$ for both samples, indicating the absence of two-magnon processes at the extreme high field limit. ${ }^{52}$ As the field is reduced, one approaches a relatively narrow range of fields, referred to as the manifold region, where the driven FMR mode is degenerate with moderate wave number (momentum) spin waves with wavelengths on the order of a micron. In this manifold region, coupling to spin modes, i.e. two-magnon scattering, dominates the relaxation in polycrystalline ferrites, ${ }^{43,48}$ and the measured effective linewidths are significantly larger (as large as 30 
Özgür et al., submitted to Journal of Materials Science: Materials in Electronics, 2009 Microwave Ferrites, Part 1: Fundamental properties

Oe for hipped YIG) than those outside the manifold region. As the field is lowered further, the linewidth reduces again, but it is generally higher than that of the high field effective linewidth. Figure 17 shows the effective linewidth measured for polycrystalline YIG. $^{53}$

These linewidths have been studied also for ferrite thin films. It has been demonstrated that high quality epitaxial films with microwave losses equal to those in bulk crystals can be produced. ${ }^{54}$ For example, single crystal LiZn ferrite films $(0.7 \mu \mathrm{m})$ grown on $\mathrm{MgO}$ substrates by PLD were shown to exhibit FMR linewidths as low as 57 Oe at $9.5 \mathrm{~Hz} .^{55}$ An FMR linewidth of 27 Oe was measured at $60 \mathrm{GHz}$ for $45 \mu \mathrm{m}$-thick BaM films grown by liquid phase epitaxy on $\mathrm{MgO}$ substrates. ${ }^{56}$ For $0.85 \mu$ m-thick $\mathrm{BaFe}_{12} \mathrm{O}_{19}(\mathrm{BaM})$ films deposited by PLD on sapphire substrates the main mode half power FMR linewidth was $27 \mathrm{Oe}$ at $60.3 \mathrm{GHz}$ and decreased linearly with frequency by $0.5 \mathrm{Oe} / \mathrm{GHz}$ with a zero linewidth intercept at zero frequency. ${ }^{54}$ Figure 18 (a) shows the absorption derivative versus the static applied field near the main FMR. Assuming a Lorentzian form for the absorbed power, one would expect the separation between the two extrema in the absorption derivative to be $\Delta H / \sqrt{3}$, where $\Delta H$ is the FMR linewidth, which gives a value of 16 Oe as indicated in Figure $18(\mathrm{a}) .{ }^{54}$ The half power FMR linewidth as a function of the frequency is shown in Figure 18(b) for PLD grown and bulk BaM samples. It is evident that the thin film linewidth matches that of the bulk single crystal. ${ }^{57}$ The FMR linewidth response of $0.5 \mathrm{Oe} / \mathrm{GHz}$ in $\mathrm{BaM}$ is 10 times larger than that for single crystal YIG, which is typically $0.05 \mathrm{Oe} / \mathrm{Hz}$. If only intrinsic losses are assumed to 
Özgür et al., submitted to Journal of Materials Science: Materials in Electronics, 2009 Microwave Ferrites, Part 1: Fundamental properties

be affecting the FMR linewidth, such losses in BaM are considered to be much higher than in YIG.

To summarize this section, it should be noted that increasing demands for low loss ferrite materials for high frequency applications continually fuel the research on fundamental understanding of microwave loss. FMR and effective linewidth measurements have proven to be the fundamental basis for evaluation of on-resonance and off-resonance losses in ferrites. Elimination of imperfections and impurities in ferrite materials by optimizing the growth processes using advanced deposition techniques is key to achieving materials with only purely intrinsic losses.

\section{Properties of Ferrites}

While the awareness on naturally occurring magnetic materials goes really way back (the $6^{\text {th }}$ Century B.C.), production of synthesized magnetic materials began in 1930 s with alnicos, which are cubic in structure and sport a high Curie temperature $\left(\sim 850^{\circ} \mathrm{C}\right)$. A shortcoming of these magnetic materials, however, is that they are soft with rather low intrinsic coercivities $\left(\sim 50-130 \mathrm{kAm}^{-1}\right)$ which make these magnets less resistant to demagnetization. We should mention that alnico compounds were continually developed and the coercivity improved.

Despite continual improvement on the part alnico alloys in terms of the coercive field, to combat the easy demagnetization, hexagonal hard ferrites with relatively large coercivity 
Özgür et al., submitted to Journal of Materials Science: Materials in Electronics, 2009 Microwave Ferrites, Part 1: Fundamental properties

$\left(\sim 250 \mathrm{kAm}^{-1}\right)$ were developed in 1950s. Hexagonal ferrite structures are found in e.g. $\mathrm{BaO} \cdot 6 \mathrm{Fe}_{2} \mathrm{O}_{3}$ and $\mathrm{SrO} \cdot 6 \mathrm{Fe}_{2} \mathrm{O}_{3}$. Replacing $\mathrm{Ba}$ with $\mathrm{Sr}$ leads to slightly superior magnetic properties. Because of their large crystallographic anisotropy, caused in part by crystallographic distortion induced by $\mathrm{Ba}$ or $\mathrm{Sr}$, they exhibit large coercivity and substantial resistance to demagnetization, but the energy product $\left(\mathrm{BH}_{\max }\right)$ is low. With hexaferrites, applications such as permanent magnets in electric motors became a reality. These ferrites are also conducive for high frequency microwave passive components and will soon get the discussion it deserves.

The year 1960 and the years that followed experienced substantial advances in magnetic materials containing rare earths, such as yttrium cobalt $\left(\mathrm{YCo}_{5}\right)$, which makes use of the phase based on a rare-earth (RE) and a transition metal (TM). The combination of RE and $\mathrm{TM}$ is ideal as the RE element provides the anisotropy to the phase and the TM material leads to high magnetization with large energy product and high Curie temperature. This development was soon followed by the discovery of $\mathrm{SmCo}_{5}$ in 1967 , becoming the first commercial RE/TM permanent magnetic material.

This above mentioned development was soon followed by the discovery of samarium cobalt $\left(\mathrm{SmCo}_{5}\right)$ in 1967 , becoming the first commercial $\mathrm{RE} / \mathrm{TM}$ permanent magnetic material. Fast forwarding, a variety of soft ferrites was then developed. However, the metallic soft magnetic materials cannot be used at high frequencies due to Eddy current losses. For this purpose, ceramic insulator based soft ferrites became the desirable material. As previously discussed, these materials are ferrimagnetic with a cubic crystal 
Özgür et al., submitted to Journal of Materials Science: Materials in Electronics, 2009 Microwave Ferrites, Part 1: Fundamental properties

structure and the general composition of the type $\mathrm{MeO} \cdot \mathrm{Fe}_{2} \mathrm{O}_{3}$, where Me specifies a transition metal such as nickel, manganese or zinc. MnZn ferrite can be used at frequencies up to $10 \mathrm{MHz}$, for example in telephone signal transmitters and receivers and in switch mode power supplies (also referred to as DC-DC converters). Additionally, a part of the family of soft ferrites is the microwave ferrites, which are used in the frequency range from $100 \mathrm{MHz}$ to $170 \mathrm{GHz}$ (upper end of the D band), for waveguides for electromagnetic radiation and for passive microwave devices such as phase shifters, isolators, and circulators to name a few.

Microwave ferrites are magnetic ceramic materials that refer to the entire family of iron oxides which includes spinels, garnets, hexaferrites, and orthoferrites. ${ }^{58,5960,61}$ A list of the most studied ferrites with different crystal structures and some of their physical properties are presented in Table $8 .{ }^{62,63,64,65,66,67,68}$ Spinel structure materials are cubic and have the form $\mathrm{AB}_{2} \mathrm{O}_{4}$ where $\mathrm{A}$ represents divalent cations and $\mathrm{B}$ - trivalent cations of Fe. The exchange interaction between $\mathrm{A}$ and $\mathrm{B}$ sites is negative and the strongest among the cations so that the net magnetization comes from the difference in magnetic moment between A and B sites (see Figure 10). Hexagonal ferrites, or hexaferrites, have an even more complicated crystal structure (see Figure 12). There are different types of hexaferrites in terms of their composition. However, all of them share a similar crystal structure as discussed in Section 3 and will be reiterated below to a lesser extent for convenience. The Fe cations are the sole source of magnetic moment and can be found on any of the three crystallographically different sites making up the hexaferrites: tetrahedral, octahedral, and hexahedral sites. The garnets, like the spinels, also have a cubic structure 
Özgür et al., submitted to Journal of Materials Science: Materials in Electronics, 2009 Microwave Ferrites, Part 1: Fundamental properties

but with trivalent cations (including rare earth and $\mathrm{Fe}^{3+}$ ) occupying tetrahedral (A), octahedral (B), or dodecahedral (C) sites. The interaction between tetrahedral and octahedral sites is antiparallel, and the net magnetic moment is antiparallel to the rare earth ions on the $\mathrm{C}$ sites.

Rare earth orthoferrites are also classified as ferrites, although they are canted antiferromagnets. They have an orthorhombic crystal structure and the general chemical formula is $\mathrm{AFeO}_{3}$ where $\mathrm{A}$ is yttrium or a rare earth. The magnetic structure inside of a sublattice is usually collinear ferromagnetic, but the different sublattices are coupled antiferromagnetically. Due to the different number of magnetic ions in different sublattices, there is a net resulting magnetic moment, giving rise to ferrimagnetism. The nature of the superexchange interaction depends not only on the type of the magnetic ion, but rather strongly on the bond length and bonding angle. This makes it possible to change the strength and type of the superexchange interaction, i.e. the magnetization and the Néel point, by chemical substitutions of different size ions.

\subsection{Hexaferrites}

There are a number of hexaferrites, such as $\mathrm{M}, \mathrm{U}, \mathrm{X}, \mathrm{Y} \mathrm{Z}$, and $\mathrm{W}$-type hexaferrites as described in Section 3.4, with the M-type variety being of the most interest. Since their discovery at the beginning of 1950s ${ }^{12,15}$ the M-type hexaferrites have continually made inroads is applications such as permanent magnets, plastoferrites, injection-molded pieces, microwave devices, and magnetic recording media. These pioneering achievements gave 
Özgür et al., submitted to Journal of Materials Science: Materials in Electronics, 2009 Microwave Ferrites, Part 1: Fundamental properties

way to comprehensive investigations of the spinel and hexagonal ferrite systems, and inspired research efforts in international scale. The interest is fuelled by their low cost, relatively high coercivity, excellent chemical stability, and corrosion resistance. Although the magnetization and coercivity of these ferrites are lower than that of the rare earth compounds these materials represent more than half the permanent magnet market.

Many attempts have been made to improve the magnetic properties of hexaferrites by doping and substitutions within the complex structure, for the A site ions as well as for the $\mathrm{Fe}$ ions. However, most types of substitutions cause a decrease of the saturation magnetization, or a drastic reduction of the magnetocrystalline anisotropy, and decrease of coercivity. Most of the commercially available barium ferrites are hard magnetic materials that originate from the magnetocrystalline anisotropy of $\mathrm{Fe}^{3+}$ in the magnetoplumbite structure as touched upon earlier. They are designed mainly for permanent magnets and perpendicular recording media. The physical properties of hexaferrites vastly vary from one to another. A particular phase which is most suitable for the application in hand can be fulfilled by choosing the most appropriate phase and carrying out the adequate cation substitutions. The hexaferrites have been used as permanent magnetic materials owing to their excellent intrinsic hard properties. Unfortunately, the formation of ferrites requires a high temperature, $>900{ }^{\circ} \mathrm{C}$, that creates impediments in integration microelectronics industry. Unlike a magnetic metal, a ferrite is a magnetic dielectric that allows an electromagnetic wave to penetrate the ferrite, thereby permitting interaction between the wave and magnetization within the ferrite which form the basis for a variety of useful devices. The great diversity of ferrites permits 
Özgür et al., submitted to Journal of Materials Science: Materials in Electronics, 2009 Microwave Ferrites, Part 1: Fundamental properties

one to cover a wide range of frequencies (from $\mathrm{kHz}$ to tens of $\mathrm{GHz}$ ) for a set of appropriate properties (e.g., permeability, saturation magnetization, conductivity). For example, in microwave systems that use a single antenna aperture for both sending and receiving, the component at the heart of the system is a ferrite based circulator which primarily facilitates the routing of outgoing and incoming signals to the transmitter or receiver as appropriate.

Development of microwave devices requires understanding of properties of ferrites and their interaction with RF magnetic field that can be expressed by the permeability tensor, which was discussed in Section 4.1. The permeability tensor was first derived by Polder ${ }^{35}$ in 1949 which set the basis for understanding ferrite behavior at microwave frequencies. The permeability tensor $\mu$ and the susceptibility tensor $\chi$ can be obtained from the equation of motion of magnetic dipole in the presence of both a static magnetic and a transverse RF magnetic field as described in Section 4. As previously mentioned, the magnetization in ferrites originates from the spin moment of the magnetic electron. The static magnetic field aligns the magnetic moment of the electron $\mathbf{m}$ along the field direction to minimize its potential energy. The RF magnetic field that is applied perpendicular to the static field causes the magnetization to precess around the equilibrium direction with the angular frequency of the RF field (see Figure 13). The physical properties of the above mentioned hexaferrites vary greatly from one another. The particular phase needed for the application in hand can be fulfilled by choosing the most appropriate phase and carrying out adequate cation substitutions. 
Özgür et al., submitted to Journal of Materials Science: Materials in Electronics, 2009 Microwave Ferrites, Part 1: Fundamental properties

\subsubsection{M-type Hexaferrites}

We learned so far that the most commonly used type of hexaferrites, M-type hexaferrites, refer to a group of ferrimagnetic oxides having a chemical composition of $\mathrm{AFe}_{12} \mathrm{O}_{19}$ and a hexagonal crystal structure, where $\mathrm{A}$ is a divalent cation such as $\mathrm{Ba}^{2+}, \mathrm{Sr}^{2+}$ or $\mathrm{Pb}^{2+}$. It is common to use Me for A where Me represents the metal cation. A salient feature of Mtype hexaferrites is the large uniaxial anisotropy inherent in the hexagonal crystal structure, where the crystallographic $c$-axis is the magnetic easy axis. As mentioned in Section 3.4, the best-known member of the M-type hexaferrites is the uniaxial permanent magnet $\mathrm{BaFe}_{12} \mathrm{O}_{19}$ (Ferroxdure) with high anisotropy, which paves the way for a large spin-orbit coupling. The FMR linewidths of M-type hexaferrites are much larger than those of YIG, i.e. up to $\Delta H=50$ Gauss for the best material against $\Delta H=0.5$ Gauss for YIG. With proper design and substitutions passive elements including mm-wave circulators can be constructed from hexaferrites. As discussed in Section 3.4, the net magnetization of $\mathrm{BaFe}_{12} \mathrm{O}_{19}$ per molecular unit is, $M_{n e t}=(1-2+7-2) 5 \mu_{\mathrm{B}}=20 \mu_{\mathrm{B}}$.

M-type hexaferrites have two types of anisotropy, $c$-axis anisotropy and $c$-plane anisotropy, which are associated with the easy magnetization along the $c$-axis and in the $c$-plane, respectively. The magnetic field required for rotation of magnetization vectors from [001] to [001] directions for $c$-axis anisotropy, is defined as the anisotropy field $H_{a}$. For $c$-plane anisotropy, there are six easy directions separated by $60^{\circ}$ from each other. The magnetization vectors can be rotated by magnetic field from one to another easy magnetization direction in two ways: through the $c$-axis or in the $c$-plane. The 
Özgür et al., submitted to Journal of Materials Science: Materials in Electronics, 2009 Microwave Ferrites, Part 1: Fundamental properties

corresponding magnetic fields required are defined as anisotropy fields $H_{\theta}$ and $H_{\phi}$, respectively, for the $c$-plane anisotropy.

M-type hexaferrite $\left(\mathrm{BaFe}_{12} \mathrm{O}_{19}\right.$ or $\left.\mathrm{BaM}\right)$ films can be deposited by various vacuum deposition techniques such as evaporation, ${ }^{69,70}$ metalorganic chemical vapor deposition (MOCVD), ${ }^{71}$ rf sputtering, ${ }^{72,73,74}$ and pulsed laser deposition (PLD) ${ }^{54}$ sol-gel techniques, ${ }^{75,76}$ and liquid phase epitaxy (LPE) deposition technique. ${ }^{19,56,77}$ Such films are amorphous and need post deposition thermal annealing. ${ }^{78,79}$ The annealing conditions govern the crystallization and the magnetic properties obtained are strongly linked to the elaboration parameters. As a substrate a wide range of materials such as sapphire $\left(\mathrm{Al}_{2} \mathrm{O}_{3}\right),{ }^{80,81,82}$ (111) magnesium oxide $(\mathrm{MgO}),{ }^{56,83} \mathrm{SiO}_{2}, \mathrm{ZnO}$, and $\mathrm{GaN}^{84}$ are used. A comparative study of barium hexaferrite films growth on four different substrates, $\mathrm{SiO}_{2}$, $\mathrm{ZnO}, \mathrm{Gd}_{3} \mathrm{Ga}_{5} \mathrm{O}_{12}$, and $\mathrm{Al}_{2} \mathrm{O}_{3}$ by a target-facing sputtering system has been undertaken by Lacroix et al. ${ }^{85}$ On amorphous silica Ba affinity for $\mathrm{SiO}_{2}$ resulted in the formation of an intermediate glass layer with high $\mathrm{Ba}$ and $\mathrm{Si}$ concentrations. This gave way to (111) spinel which is magnetite, and finally to the well $c$-oriented Ba hexaferrite with $\Delta \omega=2.3^{\circ}$. With progressive annealing, the magnetite transformed into maghemite and then into hexaferrite. On (001) $\mathrm{ZnO}$, another (111) spinel, $\mathrm{ZnFe}_{2} \mathrm{O}_{4}$, is formed on which textured Ba hexaferrite film is grown $\left(\Delta \omega=1.3^{\circ}\right)$. On gadolinium gallium garnet $\left(\mathrm{GGG}, \mathrm{Gd}_{3} \mathrm{Ga}_{5} \mathrm{O}_{12}\right.$ which is cubic) and $\mathrm{Al}_{2} \mathrm{O}_{3}$ (which is hexagonal) there were no intermediate (interfacial) layers. In the former case a well $c$-axis-oriented $\mathrm{Ba}$ hexaferrite with $\Delta \omega=3.6^{\circ}$ was observed whereas for the latter case the orientation of the hexaferrite was found to be random. Yoon and Vittoria ${ }^{19}$ reported on the growth of thick barium hexaferrite 
Özgür et al., submitted to Journal of Materials Science: Materials in Electronics, 2009 Microwave Ferrites, Part 1: Fundamental properties

$\mathrm{BaFe}_{12} \mathrm{O}_{19}$ films on $m$-plane sapphire $\left(\mathrm{Al}_{2} \mathrm{O}_{3}\right)$ substrates by a modified liquid phase epitaxy deposition technique which was preceded by $\mathrm{BaFe}_{12} \mathrm{O}_{19}$ seed layer deposition on the substrate by pulsed laser ablation. Films with the total thickness ranging from $60 \mu \mathrm{m}$ to $200 \mu \mathrm{m}$ were grown. The vibrating sample magnetometer measurement data showed magnetic uniaxial anisotropy axis of the film. The coercive field $H_{c}$ for these particular films was relatively small, ranging from $0.007 \mathrm{kOe}$ to $0.08 \mathrm{kOe}$. A saturation magnetization of $4.42 \mathrm{kG}$ and a ferrimagnetic resonance linewidth $(\Delta H)$ of $\sim 0.08 \mathrm{kOe}$ at $59.9 \mathrm{GHz}$ were demonstrated.

As already alluded to, the magnetic properties of hexaferrites can be controlled by doping. For example, the magnetization and the anisotropy of Ba-ferrite can be increased by substitution of $\mathrm{Al}^{3+}, \mathrm{Ga}^{3+}$ or $\mathrm{Cr}^{3+}$; while $\mathrm{Ti}^{4+} \mathrm{Me}^{2+}$ or $\mathrm{Ge}^{4+} \mathrm{Me}^{2+}\left(\mathrm{Me}^{2+}=\mathrm{Zn}, \mathrm{Co}, \mathrm{Ni}\right)$ substitution decreases the magnetization and the anisotropy field. In order to lower the coercivity $\left(H_{\mathrm{c}}\right)$, decrease the positive temperature coefficient of $H_{\mathrm{c}}$ and obtain fine particle size, the divalent-tetravalent metal elements $\mathrm{La}-\mathrm{Co}{ }^{86,87,88,89}, \mathrm{Ti}-\mathrm{Co}{ }^{90,91,92}, \mathrm{Zn}-$ $\mathrm{Co}^{93}, \mathrm{Zn}-\mathrm{Ti}{ }^{94,95}, \mathrm{Ir}-\mathrm{Co}{ }^{92,96}, \mathrm{Gd}-\mathrm{Co},{ }^{97}$ and $\mathrm{Ni}-\mathrm{Zr}^{98}$ have been used. On the other hand, in order to increase the $H_{\mathrm{c}}$ of the hexaferrites for the fabrication of permanent magnets, trivalent metal elements such as $\mathrm{La}, \mathrm{Es}, \mathrm{Si}, \mathrm{Ga}, \mathrm{In}, \mathrm{Co}, \mathrm{Bi}, \mathrm{Sb}$ and $\mathrm{Al}$ have also been reported by many authors $99,100,101,102,103,104,105,106,107,108,109$. It should be noted, however, that as a consequence of these substitutions, the saturation magnetization is decreased. It was found, however, that quite generally either the saturation magnetization $M_{s}$, or the anisotropy field $H_{a}$, or both decrease. 
Özgür et al., submitted to Journal of Materials Science: Materials in Electronics, 2009 Microwave Ferrites, Part 1: Fundamental properties

A make or break type property is the magnetic anisotropy. In order to enhance magnetocrystalline anisotropy in substituted $\mathrm{Sr}_{1-\mathrm{y}} \mathrm{La}_{\mathrm{y}} \mathrm{Fe}_{12-\mathrm{x}} \mathrm{Co}_{\mathrm{x}} \mathrm{O}_{19}$ ferrites Wiesinger et $a l .{ }^{88}$ carried out ${ }^{57} \mathrm{Fe}$ Mössbauer and ${ }^{57} \mathrm{Fe}$ NMR studies. From the experiments a preferred $\mathrm{Co}^{2+}$ occupation on the $\mathrm{Fe}^{3+}$ lattice sites was derived. It was also found that $\mathrm{Co}^{2+}$ preferably occupies the $4 f_{2}$ sublattice. Mössbauer spectroscopy indicated a certain possibility that $\mathrm{Co}^{2+}$ occupies the 2 a sites as well, while NMR studies appear to be in favor of the $4 \mathrm{fl}$ site occupation by $\mathrm{Co}^{2+}$ ions. Furthermore, in samples with $\mathrm{x} \geq 0.1$ the ${ }^{57} \mathrm{Fe}$ Mössbauer spectroscopy proved the presence of a pattern which is attributed to $\mathrm{Fe}^{2+}$ on the $2 a$ sites.

The $\mathrm{La}^{3+}$ substitution for $\mathrm{Ba}^{2+}$ in $\mathrm{BaFe}_{12} \mathrm{O}_{19}$ was reported to result in the iron valence changing from $\mathrm{Fe}^{3+}$ to $\mathrm{Fe}^{2+} \cdot{ }^{110}$ Furthermore, La-doping of hexaferrite was found to produce a relatively high magneto-crystalline anisotropy at room temperature. However, phase equilibrium studies of $\mathrm{La}-\mathrm{Fe}-\mathrm{O}$ system in air indicated that the $\mathrm{La} \mathrm{Fe}_{12} \mathrm{O}_{19}$ phase is only truly stable in the range from 1380 to $1421^{\circ} \mathrm{C}$, which has been attributed to the change in the concentration and site occupancy of the $\mathrm{Fe}^{3+}$ ion due to the partial substitution by $\mathrm{Fe}^{2+} \cdot 111$ As a result the synthesis of single phase $\mathrm{LaFe}_{12} \mathrm{O}_{19}$ was found to be difficult. In addition, $\mathrm{LaFeO}_{3}$ and $\mathrm{Fe}_{3} \mathrm{O}_{4}$ phases were also formed. However, a partial substitution of $\mathrm{La}$ and/or $\mathrm{La}-\mathrm{Co}$ into $\mathrm{Sr}$ hexaferrite $\left(\mathrm{SrFe}_{12} \mathrm{O}_{19}\right.$ or $\left.\mathrm{SrM}\right)$ is possible and was reported to improve significantly the magnetic properties. ${ }^{107,108}$ An earlier report ${ }^{86}$ showed improved properties by simultaneous doping with La and Co. 
Özgür et al., submitted to Journal of Materials Science: Materials in Electronics, 2009 Microwave Ferrites, Part 1: Fundamental properties

The effects of the initial $\mathrm{La} / \mathrm{Sr}$ ratio on the structure, particle morphology and magnetic properties of La-substituted Sr hexaferrite have been investigated by Wang et al. ${ }^{108}$ Lasubstituted Sr hexaferrite particles in this work were prepared by hydrothermal synthesis and subsequent calcinations. Employing x-ray diffraction, scanning electron microscopy, vibrating sample magnetometry and pulsed field magnetometer measurements it was found that under hydrothermal conditions the $\mathrm{La}^{3+}$ additives did not substitute exclusively into the SrM structure, but this was achieved by subsequent calcinations at high temperatures. La substitution increased slightly the coercivity for the samples with $\mathrm{La} / \mathrm{Sr}$ ratios up to $1 / 8$, however, for higher $\mathrm{La} / \mathrm{Sr}$ ratios, such as $1 / 4$ and $1 / 2$, the coercivity was smaller than that for the sample without La. This observation was attributed to grain growth during calcination. Most of the La-substituted samples exhibited a similar magnetization to that of $\mathrm{SrFe}_{12} \mathrm{O}_{19}$.

Sm and $\mathrm{Nd}$ additives to $\mathrm{Sr}_{1-\mathrm{x}} \mathrm{Nd}_{\mathrm{x}} \mathrm{Fe}_{12} \mathrm{O}_{19}$ ( $\mathrm{SrM}$ ) has also been reported to lead to significant improvements in the coercivity. ${ }^{109} \mathrm{Nd}$-substituted $\mathrm{Sr}$ hexaferrite plate-like particles were synthesized hydrothermally and then calcined at temperatures ranging from $1100{ }^{\circ} \mathrm{C}$ to $1250{ }^{\circ} \mathrm{C}$ for $2 \mathrm{~h}$ in air. $\mathrm{Nd}$ substitution up to a $\mathrm{Nd}-\mathrm{Sr}$ ratio of $1 / 8$ increased the coercivity without causing any significant deterioration in either the saturation magnetization or the remanence.

The effect of zinc and niobium doping on magnetic properties of strontium hexaferrite $\operatorname{Sr}\left(\mathrm{Zn}_{0.7} \mathrm{Nb}_{0.3}\right)_{x} \mathrm{Fe}_{12-x} \mathrm{O}_{19}(x=0-1.0)$ nanoparticles was investigated by Fang et al. ${ }^{75}$ in solgel produced material. The data indicated that for low $\mathrm{Zn}$ and $\mathrm{Nb}$ concentrations the 
Özgür et al., submitted to Journal of Materials Science: Materials in Electronics, 2009 Microwave Ferrites, Part 1: Fundamental properties

strontium hexaferrite retained its hexagonal magnetoplumbite phase. The coercive force was found to reduce from 6.7 to about $2.3 \mathrm{kOe}$, while the values of saturation magnetization increased to $67-74 \mathrm{emu} / \mathrm{g}$ for the substitution range of $x=0-1.0$ (Figure 19). This indicates that the saturation magnetization and coercivity of strontium hexaferrite nanoparticles can be retained over a very wide range by an appropriate amount of $\mathrm{Zn}$ and $\mathrm{Nb}$ doping. Moreover, it was observed that the doped strontium hexaferrite nanoparticles show a small positive temperature coefficient of coercivity. The substitution of $\mathrm{Zn}^{2+}$ and $\mathrm{Nb}^{4+}$ ions for $\mathrm{Fe}^{3+}$ ions also affects the temperature dependence of magnetization and Curie temperature, and enhances thermal stability at least in the measured temperature range.

As for the electronic properties, first-principles band structure calculations for $\mathrm{SrFe}_{12} \mathrm{O}_{19}$ using density functional theory within the local spin density approximation performed by Fang et al. ${ }^{112}$ showed that the $\mathrm{Fe}^{3+}$ ions are almost fully spin polarized with high spin states $(S=5 / 2)$. It was noted that the electronic structure was influenced substantially by the exchange interaction between the $\mathrm{Fe}^{3+}$ ions. The most stable form of the hexaferrite is a ferrimagnet with the $\mathrm{Fe}^{3+}$ ions situated at the $4 \mathrm{f}$ sites with their spins polarized antiparallel to the remaining $\mathrm{Fe}^{3+}$ ions. This hexaferrite is predicted to be a semiconductor with Fe $3 d$ states dominating both the top of the valence band and the bottom of the conduction band. Moreover, a strong anisotropy was noted for the conductive charge carriers. The suggested bandgap was $0.63 \mathrm{eV}$ with experimental values expected to be much larger as many of these computational methods are mired with difficulties in predicting the bandgaps properly. 


\subsubsection{Variants of hexaferrites}

In addition to M-type hexaferrites other cousins, namely $\mathrm{U}, \mathrm{X}, \mathrm{Y}, \mathrm{Z}$ and $\mathrm{W}$-type hexaferrites, with more complex structures have been prepared. In the pioneering investigations of Jonker et al., ${ }^{15}$ three types of complex hexaferrites along with their composition and crystal structure were determined and the notation $\mathrm{MeW}=$ $1 \mathrm{BaO} \cdot 2 \mathrm{MeO} \cdot 8 \mathrm{Fe}_{2} \mathrm{O}_{3}, \mathrm{MeY}=2 \mathrm{BaO} \cdot 2 \mathrm{MeO} \cdot 6 \mathrm{Fe}_{2} \mathrm{O}_{3}$, and $\mathrm{MeZ}=3 \mathrm{BaO} \cdot 2 \mathrm{MeO} \cdot 12 \mathrm{Fe}_{2} \mathrm{O}_{3}$ was coined to describe them. Here Me stands for elements such as $\mathrm{Co}, \mathrm{Cu}, \mathrm{Fe}, \mathrm{Mg}$, Ni or Zn. The aforementioned Me substitutions have a distinct effect on magnetic anisotropy and the microwave linewidth of the FMR. Naturally, the key here is to find a combination giving rise to large anisotropy and small FMR linewidth to be useful in microwave components operational above $10 \mathrm{GHz}$. The W- and Z-type hexaferrites exhibit planar anisotropy only if they contain a substantial proportion of cobalt in their composition. Less than the critical amount of cobalt causes the easy direction of magnetization (uniaxial) to be parallel to the c-axis. It should be mentioned that divalent ions other than Co, such as $\mathrm{Zn}^{2+}, \mathrm{Fe}^{2+}$, and $\mathrm{Ni}^{2+}$, can lead to easy $c$-axis anisotropy for both $\mathrm{W}$ - and $\mathrm{Z}$ type hexaferrites. ${ }^{13,114}$ As in the previous cases, Y-hexaferrites and their hexaferrites containing Co exhibit planar magnetocrystalline anisotropy. ${ }^{114}$

The $\mathrm{Y}-$, Co- $\mathrm{U}, \mathrm{Co}-\mathrm{M}$ and $\mathrm{Co}-\mathrm{Z}$ hexaferrites have been considered for microwave absorbers. ${ }^{115,116}$ As discussed in Section 3.4, their crystalline structure ${ }^{117}$ is comprised by three basic structural blocks: namely $\mathrm{S}=\mathrm{Fe}_{6} \mathrm{O}_{8}$ (spinel), $\mathrm{R}=\mathrm{BaFe}_{6} \mathrm{O}_{11}$ (hexagonal), and 
Özgür et al., submitted to Journal of Materials Science: Materials in Electronics, 2009 Microwave Ferrites, Part 1: Fundamental properties

$\mathrm{T}=\mathrm{Ba}_{2} \mathrm{Fe}_{8} \mathrm{O}_{14}$ (hexagonal) in such a way that a specific combination of these blocks results in a specific hexaferrite type, e.g. $(\mathrm{RS})_{2}=\mathrm{M},(\mathrm{TS})_{3}=\mathrm{Y}$, and $(\mathrm{RSTS})_{2}=\mathrm{Z}$. There are of course other types of hexaferrites such as W, X or U (see Table 5). Co-U hexaferrites possess a very similar structure, $(\mathrm{RSRSTS})_{3}$, to that of $\mathrm{Co}-\mathrm{Z}$ hexaferrites.

A variety of X-type hexaferrites has been experimentally investigated. $^{93,118,119,120,121,122,123}$ As alluded to earlier in Section 3.4, X-type hexaferrite is one whose structure can be construed as a stack of hexagonal R- and spinel (cubic) Sblock along the hexagonal $c$-axis with a model as RSR*S*S*, with the asterisk indicating that the corresponding block is rotated by $180^{\circ}$ around the hexagonal axis. Magnetization, magnetic moment, and the Curie temperature of $\mathrm{X}$-type hexaferrite $\mathrm{Ba}_{2} \mathrm{Zn}_{x} \mathrm{Co}_{2-x} \mathrm{Fe}_{28} \mathrm{O}_{46}$ have been investigated and reported in references 93 and 118 and the results showed linear increase of the magnetization and magnetic moment with increasing zinc content. $\mathrm{Gu}^{119,120}$ studied low temperature magnetic properties and distribution of divalent cation within $\mathrm{Ba}_{2} \mathrm{Me}_{2} \mathrm{Fe}_{28} \mathrm{O}_{46}(\mathrm{Me}=\mathrm{Fe}, \mathrm{Co}, \mathrm{Mn}, \mathrm{Ni}, \mathrm{Cu}, \mathrm{Mg}, \mathrm{Zn})$, the results indicate that the substitution of $\mathrm{Fe}^{2+}$ ion with $\mathrm{Ni}^{2+}, \mathrm{Cu}^{2+}, \mathrm{Mg}^{2+}, \mathrm{Co}^{2+}$ or $\mathrm{Mn}^{2+}$ causes a decrease of the spontaneous moment $m$, while the substitution of $\mathrm{Zn}^{2+}$ ion increases the $m$, which was attributed to the preference of the first group of ions for octahedral sites in the S-block, while $\mathrm{Zn}^{2+}$ ion prefer the tetrahedral sites.

Quite a few investigations on the preparation, albeit the least among all the hexaferrites, characterization, and magnetic properties of U-type hexaferrite $\mathrm{BaMe}_{2} \mathrm{Fe}_{36} \mathrm{O}_{60}$ have been undertaken. $^{124,125,126,127,128,129}$ These types of ferrites are typically very difficult to prepare 
Özgür et al., submitted to Journal of Materials Science: Materials in Electronics, 2009 Microwave Ferrites, Part 1: Fundamental properties

owing to their complex crystalline structure. ${ }^{22}$ However, single-phase albeit polycrystalline U-hexaferrites with the composition $\mathrm{Ba}_{4} \mathrm{~A}_{2} \mathrm{Fe}_{36} \mathrm{O}_{60}(\mathrm{~A}=\mathrm{Co}, \mathrm{Ni}, \mathrm{Zn})$ have been successfully synthesized by solid-state reaction synthesis for which high-energy milling or topotactic reaction and calcination at $1200-1300{ }^{\circ} \mathrm{C}$ were employed. Naturally, the Curie temperature and saturation magnetization in these samples were noted as being influenced by the film composition, while the coercivity appeared to be more strongly affected by preparation conditions. ${ }^{127}$ Microwave absorption data for Co-U hexaferrites, with the composition $\mathrm{Ba}_{4} \mathrm{Co}_{2} \mathrm{Fe}_{36} \mathrm{O}_{60}$, have been reported in films prepared by highenergy milling or chemical co-precipitation followed by reaction sintering at $1250{ }^{\circ} \mathrm{C}^{130}$ The density of ceramic hexaferrite was influenced considerably in that $4.2 \mathrm{~g} / \mathrm{cm}^{3}$ was achieved when prepared with high-energy milling, and $3.6 \mathrm{~g} / \mathrm{cm}^{3}$ when prepared with chemical co-precipitation.

Y-type hexaferrites are considered for applications requiring high permeability $(\mu)$ materials at relatively lower microwave frequencies $(0 \text { to } 10 \mathrm{GHz})^{23,131,132,133,134,135,136}$ High permeability is attributed to spin rotation and domain-wall motions. The $c$-plane anisotropic barium ferrite family is of great interest for use as a low reflection material. In the barium-ferrite family, only the Y-type barium ferrite (BaY) has c-plane anisotropy, while the others have $c$-axis anisotropy. However, substitution of some elements can modify the anisotropy from $c$ axis to $c$-plane. Various mechanochemical methods have been employed to prepare $\mathrm{Y}$ - type hexaferrites, ${ }^{137}$ and the effect of particle size and concentration on microwave absorbing properties has been investigated. ${ }^{138}$ 
Özgür et al., submitted to Journal of Materials Science: Materials in Electronics, 2009 Microwave Ferrites, Part 1: Fundamental properties

W-hexaferrites are of interest owing to their potential application as permanent magnets, microwave devices, and for bubble domain memories in the form of thin monocrystalline layers epitaxially grown on nonmagnetic substrate crystals. The crystal structure and the composition of W-type hexaferrites are closely related to most studied $\mathrm{M}$-type $\mathrm{BaFe}_{12} \mathrm{O}_{19}$ (magnetoplumbite). As mentioned, the crystal structures of M- and W-type hexaferrites can be described as an alternating stacking of spinel and R-blocks in the direction of the hexagonal c-axis. The spinel blocks in the W-type structure are twice as large as in the magnetoplumbite-type structure. ${ }^{125} \mathrm{~W}$-type hexaferrites have been prepared mainly by powder glass crystallization method ${ }^{139,140,141}$ and from sol-gel precursors. ${ }^{142,143}$ Their

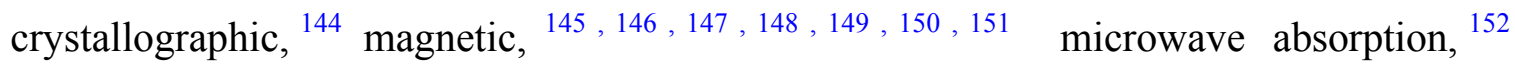
dielecrtric, ${ }^{151,153,154,155}$ and magnetostriction ${ }^{156}$ properties which are naturally dependent on the preparation method employed, have been investigated. Also two-phase M and Wtype hexaferrites have been prepared for their electromagnetic wave absorption properties. $^{152,157}$

The attractive feature of the $\mathrm{W}$-hexaferrites is that their magnetic properties can be varied in a wide range by a suitable choice of the substitutional divalent cations, without changing the iron content. For example, $\mathrm{BaZn}_{2} \mathrm{Fe}_{16} \mathrm{O}_{27}\left(\mathrm{Zn}_{2}-\mathrm{W}\right)$ is magnetically uniaxial and has the highest saturation magnetization among the hexagonal ferrites with 35 Bohr magnetons per formula unit at $0 \mathrm{~K} .{ }^{113}$ However, $\mathrm{Co}^{2+}$ - substituted $\mathrm{W}$-hexaferrites with sufficiently high cobalt content are magnetically planar. ${ }^{158}$ In other words, Co substitution modifies the anisotropy, changing it from $c$-axis to $c$-plane. ${ }^{144,159}$ 
Özgür et al., submitted to Journal of Materials Science: Materials in Electronics, 2009 Microwave Ferrites, Part 1: Fundamental properties

As an example, Li et al. ${ }^{24}$ studied substituted $\mathrm{Co}-\mathrm{Zn} \mathrm{BaW}$ ferrites, $\mathrm{BaZn}_{2-x} \mathrm{Co}_{x} \mathrm{Fe}_{16} \mathrm{O}_{27}$ with $x=0-2.0$. The samples were prepared by mixing hexaferrite fine powders and epoxy resin, and solidifying under a static magnetic field applied perpendicular to the sample surface. The grains were aligned along the [001] $c$-axis for $x<0.7$. The alignment direction, however, switched to [110] for $\mathrm{x}>0.7$, as determined from $\mathrm{x}$-ray diffraction measurements. As shown in Figure 20, with increasing Co substitution the value of $c$-axis anisotropy field $H_{a}$ decreases rapidly from $12 \mathrm{kOe}$ at $x=0$ to $5.0 \mathrm{kOe}$ at $x=0.5$. This is because Co ions are able to modify the anisotropy from $c$-axis to $c$-plane at $x=0.6-0.7$, above which the $c$-plane anisotropy field, $H_{\theta}$, increases from $4.5 \mathrm{kOe}$ at $x=0.7$ to $21 \mathrm{kOe}$ at $x=2.0$. For the BaW composites with $c$-plane anisotropy $(\mathrm{x}>0.7)$, the ferromagnetic resonance frequency $f_{R}$ also increases with increasing Co concentration $x$, from $2.5 \mathrm{GHz}$ at $x=0.7$ to $12.0 \mathrm{GHz}$ at $x=1.5$.

Takada et al. ${ }^{160}$ studied substitution effect of $\mathrm{Sr}^{2+}$ for $\mathrm{Ba}^{2+}$ on directions of magnetic moments and their temperature dependences in $\mathrm{Co}_{2} \mathrm{Z}$-type $\mathrm{Ba}$ ferrite $\mathrm{Ba}_{3} \mathrm{Co}_{2} \mathrm{Fe}_{24} \mathrm{O}_{41}$ $\left(\mathrm{Ba}_{3} \mathrm{Z}\right)$ with high-temperature neutron diffraction technique. They prepared three different types of samples - non-oriented and magnetically oriented specimens of $\mathrm{Co}_{2} \mathrm{Z}$ type $\mathrm{Ba}$ ferrite $\mathrm{Ba}_{3} \mathrm{Co}_{2} \mathrm{Fe}_{24} \mathrm{O}_{41}\left(\mathrm{Ba}_{3} \mathrm{Z}\right)$, and those with $\mathrm{Sr}^{2+}$ substitution for $\mathrm{Ba}^{2+}$, i.e., $\mathrm{Ba}_{1.5} \mathrm{Sr}_{1.5} \mathrm{Co}_{2} \mathrm{Fe}_{24} \mathrm{O}_{41}\left(\mathrm{Ba}_{1.5} \mathrm{Sr}_{1.5} \mathrm{Z}\right)$ and $\mathrm{Sr}_{3} \mathrm{Co}_{2} \mathrm{Fe}_{24} \mathrm{O}_{41}\left(\mathrm{Sr}_{3} \mathrm{Z}\right)$ with the conventional solidstate reaction method. From XRD and magnetization measurements it was revealed that the magnetic moments of iron and cobalt ions in $\mathrm{Ba}_{3} \mathrm{Z}$ and $\mathrm{Ba}_{1.5} \mathrm{Sr}_{1.5} \mathrm{Z}$ lie in the $c$ plane, while those in $\mathrm{Sr}_{3} \mathrm{Z}$ deviate from the $c$ plane. The change in moment direction was also temperature dependent. The Sr substitution induced the change in the distribution of 
Özgür et al., submitted to Journal of Materials Science: Materials in Electronics, 2009 Microwave Ferrites, Part 1: Fundamental properties

cobalt ions and moment directions of magnetic ions. Magnetic moments in $\mathrm{Ba}_{3} \mathrm{Z}$ and $\mathrm{Ba}_{1.5} \mathrm{Sr}_{1.5} \mathrm{Z}$ deviated from the $c$-plane when temperature was increased from 523 to $573 \mathrm{~K}$. This deviation of the magnetic moments from the c-axis, however, occurred at a much lower temperature of $50 \mathrm{~K}$ for $\mathrm{Sr}_{3} \mathrm{Z}$. This dependence of moment direction was explained by disappearance of effective moments of cobalt ions in these temperature regions.

\subsection{Spinel Ferrites}

Spinel ferrites, which have the general chemical composition $\mathrm{Me} \cdot \mathrm{Fe}_{2} \mathrm{O}_{4}$, are of interest owing to their magnetic, optical, and electronic properties. Magnetic properties are of prime interest for the purpose of this review. These properties depend on the nature of the metal ions, Me, and the method of preparation. A large number of ferrites share the spinel crystal structure, the details of which and the origin of net magnetization are discussed briefly in Section 3.3. The discussion in this section expands on the magnetization and is centered on the magnetic properties of some important ferrites with spinel crystal structure.

As mentioned previously, ferrimagnetism is a special class of antiferromagnetism in that oppositely aligned magnetic moments are of different magnitude, thereby leading to a non-zero net magnetization. Néel ${ }^{29,30}$ pointed out that magnetic moment of a ferrimagnetic material is the difference between the magnetic moments of $\mathrm{A}$ and $\mathrm{B}$ ions, occupying the tetrahedral and octahedral sites, respectively. As discussed in Section 3.3, in $\mathrm{Fe}_{3} \mathrm{O}_{4}$, which has an inverse spinel structure (see Section 3.3 for definition) with one 
Özgür et al., submitted to Journal of Materials Science: Materials in Electronics, 2009 Microwave Ferrites, Part 1: Fundamental properties

$\mathrm{Fe}^{2+}$ and two $\mathrm{Fe}^{+3}$ atoms, one $\mathrm{Fe}^{3+}$ occupies the $\mathrm{A}$ site, and one $\mathrm{Fe}^{3+}$ and one $\mathrm{Fe}^{2+}$ the $\mathrm{B}$ sites, resulting in a net magnetic moment of 4 Bohr magnetons.

Another example for inverse spinel is $\mathrm{CoFe}_{2} \mathrm{O}_{4}$ where one half of the $\mathrm{Fe}^{3+}$ ions (one half of the total Fe or one third of the total metal ions) occupies all A sites and the other half the $\mathrm{Fe}^{3+}$ ions (one third of the total metal ions) occupies the $\mathrm{B}$ sites, and all $\mathrm{Co}^{2+}$ ions occupy the other half of $\mathrm{B}$ sites. $\mathrm{ZnFe}_{2} \mathrm{O}_{4}$ is an example for normal spinel ferrites in which the zinc ions occupy the $\mathrm{A}$ sites and all $\mathrm{Fe}^{3+}$ ions occupy the $\mathrm{B}$ sites. In a ferrimagnetic spinel the $\mathrm{A}-\mathrm{B}$ magnetic interactions between the magnetic atoms on the $\mathrm{A}$ (tetrahedral) site and the $\mathrm{B}$ (octahedral) sites are much stronger than $\mathrm{A}-\mathrm{A}$ interactions and $\mathrm{B}-\mathrm{B}$ interactions within each sublattice. Besides, all spins for the same magnetic ion within a given sublattice (either A or B) are parallel to one another despite the weak negative interaction that favors the antiparallel alignment within each sublattice. As mentioned previously, the octahedral and tetrahedral sublattice magnetizations associated with the same charge magnetic ion, such as $\mathrm{Fe}^{3+}$, are antiparallel. The magnetic moments for trivalent magnetic ions occupying A and B sites in the inverse spinel fully cancel each other as the number of trivalent ions in the two sublattices is the same. Therefore, the net magnetization results from the divalent magnetic ions occupying the octahedral B sites.

Although, it does not affect the net magnetization calculated as above, the distribution of the divalent and trivalent ions over the B sites is random, and this disorder has a profound effect on both resistivity and magnetic properties, particularly in terms of losses. Conduction results when an electron "hops" from a divalent to a trivalent Fe ion (both on 
Özgür et al., submitted to Journal of Materials Science: Materials in Electronics, 2009 Microwave Ferrites, Part 1: Fundamental properties

B sites) facilitating electron flow giving rise to high conductivity. The energy required for this hopping is so low that it is thermally activated at room temperature. For example, $\mathrm{Fe}_{3} \mathrm{O}_{4}$ has a much lower resistivity than many other ferrite materials (about $10^{-3}-10^{-4} \Omega$ $\mathrm{cm}$ at room temperature) which is explained by the equivalent distribution of $\mathrm{Fe}^{2+}$ and $\mathrm{Fe}^{3+}$ ions on the octahedral sites. However, if the ferrous ion $\mathrm{Fe}^{+2}$ is replaced by another metal ion $\mathrm{Me}^{2+}$, changing the chemical formula to $\mathrm{MeO} \cdot \mathrm{Fe}_{2} \mathrm{O}_{3}$, the interchange of electrons is no longer possible between the trivalent $\mathrm{Fe}^{3+}$ and the divalent $\mathrm{Me}^{2+}$ ions at the B-sites without having the final state differ from the initial one. Indeed, by substituting a metallic ion such as $\mathrm{Ni}^{2+}$ for the ferrous ion in $\mathrm{Fe}_{3} \mathrm{O}_{4}$ (magnetite), the resistivity can be increased to as high as $\sim 10^{6} \Omega$-cm. For cobalt ferrite, $\mathrm{CoFe}_{2} \mathrm{O}_{4}$, where the divalent cobalt ions occupy half of the B sites, the resistivity is on the order of $10^{4} \Omega$-cm because the $\mathrm{Fe}^{3+}$ and $\mathrm{Co}^{2+}$ ions again cannot readily share electrons. There is a number of possible ferrites as there are many possible ion combinations on A and B sites using a wide range of metal ions such as $\mathrm{Mn}^{+2}, \mathrm{Mg}^{+2}, \mathrm{Ni}^{+2}, \mathrm{Zn}^{+2}, \mathrm{Cd}^{+2}, \mathrm{Co}^{+2}, \mathrm{Cu}^{+2}$, etc. A combination of two or more of these metal ions may also be used.

In ferrites containing strongly paramagnetic ions such as $\mathrm{Mn}, \mathrm{Co}, \mathrm{Ni}$ replacement of some of these ions with $\mathrm{Zn}$, for example in $\mathrm{Mn}_{1-\mathrm{x}} \mathrm{Zn}_{\mathrm{x}} \mathrm{Fe}_{2} \mathrm{O}_{4}$, can actually increase magnetization. This is due to the ability of the relatively small zinc ions to displace $\mathrm{Fe}^{3+}$ ions from the tetrahedral sites. Since the spin of $\mathrm{Fe}^{3+}$ ions on the tetrahedral sites is antiparallel to that on the octahedral sites, replacing $\mathrm{Fe}^{3+}$ ions with $\mathrm{Zn}^{2+}$ ions on tetrahedral sites would increases the overall magnetization. Fortunately, this ideal substitution is possible because zinc has been reported to have a large affinity for the 
Özgür et al., submitted to Journal of Materials Science: Materials in Electronics, 2009 Microwave Ferrites, Part 1: Fundamental properties

tetrahedral sites in the spinel structure. As a result bulk zinc ferrite has a "normal" ferrite structure in which $\mathrm{Zn}^{2+}$ ions almost exclusively occupy the tetrahedral or the A sites surrounded by four oxygen ions whereas $\mathrm{Fe}^{3+}$ ions occupy the octahedral or B sites, each surrounded by six oxygen ions. ${ }^{161}$ Although the bulk zinc ferrite is paramagnetic down to $10 \mathrm{~K}$ an enhanced magnetic susceptibility, hyperfine magnetic field, and higher magnetization were observed at room temperature in zinc ferrite formed by nanosized particles. ${ }^{162,163}$ These anomalous properties have been explained in the context of the occupation of the $\mathrm{B}$ sites by a fraction of the $\mathrm{Zn}^{2+}$ ions as well, with a simultaneous occupancy of the A sites by $\mathrm{Fe}^{3+}$ ions making the system partially inverted. ${ }^{164,165}$ The degree of inversion of $\mathrm{ZnFe}_{2} \mathrm{O}_{4}$ can be represented by the formula $\left[\mathrm{Zn}_{\delta} \mathrm{Fe}_{1-\delta}\right]^{\mathrm{A}}\left[\mathrm{Zn}_{1-\delta} \mathrm{Fe}_{1+}\right.$ $\left.{ }_{\delta}\right]^{\mathrm{B}} \mathrm{O}_{4}$, where $\delta$ is the inversion parameter, showing the $\mathrm{Zn}^{2+}$ and $\mathrm{Fe}^{3+}$ distribution at $\mathrm{A}$ and $\mathrm{B}$ sites within the structure. Values from $\delta=1$ (normal) to $\delta=0.21$ have been reported, ${ }^{166,167}$ the dispersion being depending on growth conditions and the synthesis methods used.

Cobalt ferrite $\left(\mathrm{CoFe}_{2} \mathrm{O}_{4}\right)$ is unique among the common ferrites in that it has the highest values of magneto-crystalline anisotropy ${ }^{168}$ and magnetostriction. ${ }^{169}$ This highly desirable property is attributed to unquenched orbital moment of $\mathrm{Co}^{2+}$ ions in the asymmetric crystalline field at the octahedral sites. The optimal structure for the enhanced magnetic properties of $\mathrm{CoFe}_{2} \mathrm{O}_{4}$ is the perfect inverse spinel. Cobalt ferrite, a well-known hard magnetic material, is also one of the candidates for high frequency applications and high-density recording media. It has a high coercivity of $5400 \mathrm{Oe}$, which makes it magnetically hard but high fields are also required to switch the magnetization. 
Özgür et al., submitted to Journal of Materials Science: Materials in Electronics, 2009 Microwave Ferrites, Part 1: Fundamental properties

Therefore, this magnetic material is considered as an exchange bias layer to stabilize the domain state of magnetically soft ferrites. It also has a moderately high saturation magnetization (about $80 \mathrm{emu} / \mathrm{g}){ }^{42}$ and a remarkable chemical stability along with mechanical hardness, which are helpful for many of such applications.

A variety of thin film growth techniques has been used to synthesize spinel ferrites including pulsed laser deposition (PLD), ${ }^{26,170,171}$ sputtering, ${ }^{172,173}$ molecular-beam epitaxy, ${ }^{174}$ sol-gel, ${ }^{27,28}$ and electron beam reactive evaporation. ${ }^{175}$ A review of epitaxial spinel thin film growth by Suzuki ${ }^{176}$ is available in the literature. One of the major limitations in obtaining single crystal ferrite films with magnetic properties similar to bulk is the substrate choice. In choosing a substrate factors such as the lattice mismatch, chemical compatibility, and similarity of thermal expansion coefficients of the film and the substrate must be taken into account. Suzuki et al. ${ }^{170,171}$ have used PLD to grow Co ferrite on a variety of substrates. Epitaxial spinel ferrite thin films of (Mn, $\mathrm{Zn}) \mathrm{Fe}_{2} \mathrm{O}_{4}$ and $\mathrm{CoFe}_{2} \mathrm{O}_{4}$ were grown on (100) and (110) $\mathrm{SrTiO}_{3}$ and $\mathrm{MgAl}_{2} \mathrm{O}_{4}$ using spinel structure buffer layers. High quality spinel ferrite films were grown at $400{ }^{\circ} \mathrm{C}$ on buffer layers that were grown and post-annealed at $600{ }^{\circ} \mathrm{C}$ and $1000{ }^{\circ} \mathrm{C}$, respectively. While (Mn,Zn) $\mathrm{Fe}_{2} \mathrm{O}_{4}$ grown directly on $\mathrm{SrTiO}_{3}$ and $\mathrm{MgAl}_{2} \mathrm{O}_{4}$ showed mediocre structural and magnetic properties, ferrite films grown on (100) and (110) $\mathrm{SrTiO}_{3}$ and $\mathrm{MgAl}_{2} \mathrm{O}_{4}$ buffered with $\mathrm{CoCr}_{2} \mathrm{O}_{4}$ exhibited excellent crystalline properties and bulk saturation magnetization values indicating the importance of lattice match and structural similarity between the film and the immediate buffer layer underlying the epitaxial layer of interest. Suzuki et $a l .^{170,171}$ reported that approximately one quarter of the substitutional Co occupies the 
Özgür et al., submitted to Journal of Materials Science: Materials in Electronics, 2009 Microwave Ferrites, Part 1: Fundamental properties

tetrahedral (type A) sites in the as-grown material, leading to magnetic properties that are not representative of the equilibrium inverse spinel structure. Post-growth annealing was required to move Co ions to B sites. ${ }^{171}$ This property of the as-grown films was ascribed to the non-equilibrium nature of the PLD method. The oxygen pressure $\left(P_{O 2}\right)$ during PLD was found to have a sizeable influence on magnetic anisotropy and film orientation of manganese ferrite $\left(\mathrm{MnFe}_{2} \mathrm{O}_{4}\right)$ films. ${ }^{26}$ The value of magnetic anisotropy fields $\left(H_{a}\right)$ exceeded $5 \mathrm{kOe}$ when films were grown at oxygen pressures below 5 mTorr. The magnetically preferred direction of $H_{a}$ was observed to align along the film plane when $P_{\mathrm{O}_{2}}<8$ mTorr or perpendicular to it when $P_{\mathrm{O}_{2}}>8$ mTorr.

$\mathrm{CoFe}_{2} \mathrm{O}_{4}$ is known for its higher magnetostriction among all the known ferrites that makes it one of the promising candidates for a variety of magnetostrictive applications. Magnetostrictive strain as high as 230 ppm has been already achieved for polycrystalline cobalt ferrite composites. ${ }^{177}$ Single-crystal $\mathrm{CoFe}_{2} \mathrm{O}_{4}$ has been grown on $\mathrm{MgO}(001)$ by oxygen-plasma-assisted molecular beam epitaxy (OPA-MBE), ${ }^{174}$ and the composition, structure, surface morphology, and magnetic properties of the films have been studied by a number of methods. The as-grown OPA-MBE material forms a perfect inverse spinel, in which all Co ions are in the $2+$ formal oxidation state, and occupy octahedral sites within the cation sublattice. The film surfaces were very flat with mean roughnesses of only a few angstroms $\AA$, and exhibited large and stable magnetic domains. The magnetic moment per unit volume was $250 \mathrm{emu} / \mathrm{cm}^{3}$ while the saturation magnetization for films having $\sim 1000 \AA$ thickness was $\sim 60 \%$ of that of bulk Co-ferrite. In addition to Co occupancy distribution in A and B sites and temperature, magnetic anisotropy was found 
Özgür et al., submitted to Journal of Materials Science: Materials in Electronics, 2009 Microwave Ferrites, Part 1: Fundamental properties

to depend also on the strain in the films. ${ }^{171}$ The strain state of the as-grown films determined the magnetic anisotropy axis due to the dominant role of the magneto-elastic energy. ${ }^{174}$ Compressive (tensile) in-plane strain was shown to produce a negative (positive) magneto-elastic energy, minimizing the total magnetic energy for an in-plane (out-of-plane) easy axis.

The advantage of the much simpler sol-gel method is in its simplicity and the lower annealing temperature necessary for the crystallization process as well as in the small grain size of the grown ferrite films by this method, which might be desired. The sol-gel method has been used for growth of cobalt ferrite films intended for high density magnetic recording. ${ }^{28,169}$ For sol-gel growth of $\mathrm{CoFe}_{2} \mathrm{O}_{4}$, appropriate amounts of $\mathrm{Co}\left(\mathrm{CH}_{3} \mathrm{CO}_{2}\right)_{2} \cdot 4 \mathrm{H}_{2} \mathrm{O}, \mathrm{Mn}\left(\mathrm{CH}_{3} \mathrm{CO}_{2}\right)_{2} \cdot 4 \mathrm{H}_{2} \mathrm{O}$, and $\mathrm{Fe}\left(\mathrm{NO}_{3}\right)_{3} \cdot 9 \mathrm{H}_{2} \mathrm{O}$ were dissolved in 2methoxyethanol and diethanolamine with an ultrasonic cleaner. ${ }^{28}$ The solution was refluxed at $70{ }^{\circ} \mathrm{C}$ for $12 \mathrm{~h}$. After each spin-coating process, the films were pyrolyzed at about 120 and $270{ }^{\circ} \mathrm{C}$ for 3 and 10 min, respectively. The thickness of a single deposition layer was $15 \mathrm{~nm}$, and the deposition was repeated to obtain the desired thickness. It should be mentioned that sol-gel method leads to relatively smaller grain sizes that those produced by other methods. Spin-coated samples were annealed at various temperatures ranging from 350 to $950{ }^{\circ} \mathrm{C}$ for $3 \mathrm{~h}$ in air to improve the crystalline quality. ${ }^{28}$ Grown cobalt ferrite films were single phase polycrystalline spinel structure without any preferred crystalline orientation and with the grain size in the range of $30-150 \mathrm{~nm}$ depending on the annealing temperature. Magnetic measurements showed that the 
Özgür et al., submitted to Journal of Materials Science: Materials in Electronics, 2009 Microwave Ferrites, Part 1: Fundamental properties

coercivity was strongly correlated with the annealing temperature and the maximum coercivity of 2720 Oe was obtained for the Co-ferrite film annealed at $950{ }^{\circ} \mathrm{C}$.

The effect of substitution of $\mathrm{Fe}$ and $\mathrm{Co}$ by $\mathrm{Mn}$ on the magnetostrictive properties of $\mathrm{Co}_{1.2} \mathrm{Fe}_{1.8} \mathrm{O}_{4}$ has been investigated by Bhame and Joy. ${ }^{169}$ This particular investigation showed that the substitution of Co by Mn enhances the magnetostriction for small values of $x$ in $\mathrm{Co}_{1.2-x} \mathrm{Mn}_{x} \mathrm{Fe}_{1.8} \mathrm{O}_{4}$ whereas the substitution of $\mathrm{Fe}$ by $\mathrm{Mn}$ decreases the magnetostriction continuously with increasing $x$ in $\mathrm{Co}_{1.2} \mathrm{Fe}_{1.8-x} \mathrm{Mn}_{x} \mathrm{O}_{4}$. Thus, the aforementioned approach presents a way to tune the magnetostriction to the desired value by effective substitution of Fe and Co by Mn.

Most significant properties of magnetic ceramic materials, namely magnetic saturation, coercivity, magnetization, and loss change drastically when dimensions are reduced to nanoscale. ${ }^{178,179}$ Reduction in size of the magnetic material leads to novel properties as compared to the bulk material due to small volume and high surface/volume ratio, and nanoscale magnetic materials are suggested for possible consideration in applications such as high-density recording, ${ }^{180}$ color imaging, ${ }^{181}$ ferrofluids, ${ }^{181,}{ }^{182}$ and magnetic refrigerators. ${ }^{178,179}$ Typical lexicon for size reduction is that it leads to novel properties as compared to the bulk material due to the small volume and the high surface/volume ratio if the surface effects can be dealt with successfully. For example, when the size of the magnetic particle is smaller than the critical size for multi-domain formation, the particle exists in a single-domain, where the domain-wall resonance is avoided, and therefore, the material could be use at higher frequencies. It has been reported that for use as high- 
Özgür et al., submitted to Journal of Materials Science: Materials in Electronics, 2009 Microwave Ferrites, Part 1: Fundamental properties

density magnetic recording materials, the grain size of ferrite particles must be less than $10 \mathrm{~nm}$ to avoid the exchange interaction between neighboring grains. ${ }^{183}$ Some of the widespread methods for preparation of ferrite nanoparticles in the literature are coprecipitation ${ }^{184,185,186}$ spray pyrolysis, ${ }^{187}$ sol-gel, ${ }^{188,189}$ hydrothermal growth, ${ }^{190,191}$ and citrate precursor technique. ${ }^{192}$

Cheng et al. ${ }^{193}$ studied the synthesis and magnetic properties of $\mathrm{Y}_{3-x} \mathrm{Gd}_{x} \mathrm{Fe}_{5} \mathrm{O}_{12}$ nanoparticles, with sizes between 34 and $67 \mathrm{~nm}$, prepared using a sol-gel method. The crystallite size obtained was observed to increase with increasing annealing temperature. The investigation of the particle size dependence of magnetic properties showed that for samples with the same Gd concentration $(x)$, the saturation magnetization (Ms) decreased as the particle size reduced due to the enhancement of the surface spin effect. Figure 21 presents the dependence of saturation magnetization (Ms) on the average particle size at room temperature for different Gd concentrations. For example, for $x=0.2$ the crystallite sizes are $37.0,56.4$, and $63.3 \mathrm{~nm}$ at different temperatures, and thus, the values of the saturation magnetization $\left(\mathrm{M}_{\mathrm{s}}\right)$ are 24.5, 25.33, and $26.19 \mathrm{emu} \mathrm{g}^{-1}$, respectively. This reduction is attributable to the higher surface to volume ratio in the smaller particles, which results in the existence of non-magnetized surface layer. Therefore, the saturation magnetization $\left(\mathrm{M}_{\mathrm{s}}\right)$ of the particles reduces with decreasing particle size. It can also be gleaned from Figure 21 that the saturation magnetization $\left(M_{s}\right)$ linearly decreases as the Gd concentration $(x)$ increases because the magnetic moments of $\mathrm{Gd}^{3+}$ ions align oppositely to the effective moments formed by the $\mathrm{Fe}^{3+}$ ions. 
Özgür et al., submitted to Journal of Materials Science: Materials in Electronics, 2009 Microwave Ferrites, Part 1: Fundamental properties

A similar dependence of the magnetization on the particle size was also observed in Ni$\mathrm{Zn}\left[{ }^{184}\right], \mathrm{Mn}-\mathrm{Zn}\left[{ }^{184}\right]$, and Mn-ferrites. ${ }^{194}$ Using a coprecipitation method, Rao ${ }^{184}$ prepared two mixed ferrite systems, $\mathrm{Ni}_{0,65} \mathrm{Zn}_{0,35} \mathrm{Fe} 2 \mathrm{O} 4(\mathrm{Ni}-\mathrm{Zn})$ and $\mathrm{Mn}_{0,75} \mathrm{Zn}_{0,18} \mathrm{Fe}_{2.07} \mathrm{O}_{4}(\mathrm{Mn}-$ $\mathrm{Zn}$ ) and the resulting ultrafine powders were heat treated at different temperatures from 200 to $800{ }^{\circ} \mathrm{C}$ to uncover particle size dependence of magnetic properties. That effort showed that with heat treatment the average particle size increased from 9.9 to $15.7 \mathrm{~nm}$ for $\mathrm{Ni}-\mathrm{Zn}$ ferrites and from 2.4 to $10.2 \mathrm{~nm}$ for $\mathrm{Mn}-\mathrm{Zn}$ ferrites, while the corresponding magnetization values increased from 9.1 to $23 \mathrm{emu} / \mathrm{g}$ for $\mathrm{Ni}-\mathrm{Zn}$ ferrites and from 7.9 to $11.7 \mathrm{emu} / \mathrm{g}$ for $\mathrm{Mn}-\mathrm{Zn}$ ferrites, respectively. On the other hand, the coercivity increased as the particle size decreased and reached a maximum at a threshold particle size and then decreased.

The coercivity $H_{\mathrm{C}}$ has been reported to increase with decreasing $\mathrm{NiFe}_{2} \mathrm{O}_{4}$ particle size as well. ${ }^{195}$ In this particular case, the $\mathrm{NiFe}_{2} \mathrm{O}_{4}$ nanoparticles were synthesized by using $\mathrm{Ni}$ and Fe nitrates and freshly extracted egg white (ovalbumin) in an aqueous medium. The variation of $H_{\mathrm{C}}$ with particle size was accounted for by the domain structure, critical diameter, and anisotropy of the crystal. A crystallite spontaneously breaks up into a number of domains in order to reduce the large magnetization energy it would have if it were a single domain.

\subsection{Garnet Ferrites}

We discussed the structural properties of spinel garnets in Section 3.2. Because of their small linewidths, giving rise to a low threshold power for the excitation of spin waves, 
Özgür et al., submitted to Journal of Materials Science: Materials in Electronics, 2009 Microwave Ferrites, Part 1: Fundamental properties

garnets can be used advantageously in nonlinear devices such as power limiters. Also to be mentioned is that yttrium iron garnet (YIG) has been the mainstay of microwave passive components for years. A number of other ferrites or garnets have also been prepared chiefly by doping or substitution and these will be discussed subsequently in relation to their device applications. Since its discovery in $1956,{ }^{196}$ the YIG ferrite is one of the most studied ferrites due to its impact in microwave device technology. YIG has a Curie temperature of $556 \mathrm{~K}$, a saturation magnetization $\left(4 \pi \mathrm{M}_{\mathrm{s}}\right)$ of $1700-1800$ Gauss (cgs units) at $300 \mathrm{~K}$. Single-crystal YIG possesses the narrowest linewidth of all materials, and thus the smallest losses, $\Delta H \approx 0.1 \mathrm{Oe}$ at $10 \mathrm{GHz}$.

The temperature dependence of the spontaneous magnetization is of importance. As the temperature increases, the magnetization of $\mathrm{C}$ sublattice decreases much more rapidly than that of A and D sublattices. As a result at some temperature the compensation point is reached where $\mathrm{C}$ sublattice magnetization is equal to and opposite to that of $\mathrm{A}-\mathrm{D}$ sublattice (see Figure 9 and the text associated with it regarding site nomenclature, etc.). With further increase of temperature the magnetization of the garnet is dominated by A-D sublattice. The temperature dependence of magnetizations for different RE garnets is shown in Figure 22. ${ }^{197}$ As can be gleaned from the figure the compensation point differs from one element to the other and depends on the magnetic moment of the $\mathrm{C}$ sublattice, since the magnetic moment of iron sublattice changes almost the same with temperature in all garnets. The compensation temperature for gadolinium garnet, for example, is equal to $295 \mathrm{~K}$, while this value for erbium garnet is about $80 \mathrm{~K}$. 
Özgür et al., submitted to Journal of Materials Science: Materials in Electronics, 2009 Microwave Ferrites, Part 1: Fundamental properties

Due to the three different crystallographic sites available for different cations and different valence states, the magnetic properties of YIG can be changed in a wide range. Substitution at the tetrahedral sites, for example, by Al or Ga can lower the magnetization to $4 \pi M_{\mathrm{s}}=300$ Gauss (in cgs), while substitution at the octahedral sites ( $\mathrm{Sc}$ ) increases $4 \pi M_{s}$ up to 1900 Gauss (in cgs). Dodecahedral substitutions by rare earths increase the anisotropy. Lattice parameters, magnetostriction, and linewidth can all be controlled by a specific combination of substituting ions. Compared to spinels, garnets are more stress sensitive; however, small Mn substitution, utilized commercially, minimizes the magnetostrictive stresses for example in (Y,Gd)IG garnets. ${ }^{198}$

For use in magneto-optical memory devices the films are required to have magnetic anisotropy perpendicular to the film plane. For this reason in many cases films are epitaxially deposited on (111) plane of a paramagnetic garnet substrate because the (111) axis of these ferromagnetic garnet materials is the axis of easy magnetization. YIG films have been grown by liquid phase epitaxy (LPE), ${ }^{199}$ reactive ion beam sputtering (RIBS), ${ }^{200}$ and rf-magnetron sputtering ${ }^{201,202,203}$, and PLD. ${ }^{204205,206}$ Studies revealed that growths of YIG in all methods have some disadvantages such as the problems as low deposition rate and large fluctuation in composition. Deposition rates have been slow due to the high binding energies of the oxidized target atoms $(1-5 \mathrm{~nm} / \mathrm{min})$. A higher deposition rate $(10.8 \mathrm{~nm} / \mathrm{min})$ has been reported, ${ }^{207}$ but high power densities and very high processing pressures (10-100 mTorr) are required. 
Özgür et al., submitted to Journal of Materials Science: Materials in Electronics, 2009 Microwave Ferrites, Part 1: Fundamental properties

To improve the deposition rate and compositional control of the films, Sung et al. ${ }^{201}$ used a modified rf-sputtering method in which two metallic targets ( $\mathrm{Y}$ and $\mathrm{Fe}$ ) were sputtered on polished (100) $\mathrm{MgO}$ and fused quartz substrates at 2.6-5.4 mTorr using Ar which was fed directly to the targets. A partial pressure differential was established between the Ar and the $\mathrm{O}_{2}$ gases by supplying $\mathrm{O}_{2}$ directly to the substrates using a ring configuration. In such a configuration, which avoided target oxidation, deposition rates as high as 4.78$10.85 \mathrm{~nm} / \mathrm{min}$ were achieved, 2-5 times larger than that with conventional methods. Unlike in single target sputtering, $\mathrm{O}_{2}$ flow rates were reduced because the rf power to the $\mathrm{Y}$ target was reduced to achieve $\mathrm{Y}: \mathrm{Fe}=3: 5$. All the resulting $\mathrm{YIG}$ films were of singlephase and the processing window was wide compared to that of films produced without a partial pressure differential.

As in any deposition method, the conditions used during PLD have sizeable influence on the eventual properties of the resulting films. The effects of substrate temperature and oxygen partial pressure, which were varied from 700 to $850{ }^{\circ} \mathrm{C}$ and 50 to 1000 mTorr, respectively, on the structure, composition, and magnetic properties of YIG films grown on (111) single-crystal gadolinium gallium garnet (GGG) substrates have been investigated. ${ }^{208}$ While epitaxial YIG films could be prepared under a wide range of deposition conditions, the films with narrow linewidth ( $\Delta H \sim 1$ Oe) were producible only at low oxygen partial pressures $\left(\mathrm{O}_{2}<250\right.$ mTorr $)$ and relatively high substrate temperatures $\left(T_{s} \geq 800{ }^{\circ} \mathrm{C}\right)$. Under all deposition conditions uniaxial anisotropy was observed and the uniaxial axis remained perpendicular to the film plane. However, at low 
Özgür et al., submitted to Journal of Materials Science: Materials in Electronics, 2009 Microwave Ferrites, Part 1: Fundamental properties

oxygen pressures the uniaxial anisotropy energy constant $K_{u}$ was negative with the maximum value of -850 Oe while at high oxygen pressures $K_{u}$ was positive.

Bismuth has been found to increase the Faraday rotation of YIG considerably, and the fully substituted composition $\mathrm{Bi}_{3} \mathrm{Fe}_{5} \mathrm{O}_{12}$ (abbreviated as $\mathrm{BIG}$ ) exhibits a Faraday rotation of $-7.2 \% / \mu \mathrm{m}$ at $633 \mathrm{~nm}$ and $300 \mathrm{~K}$ as compared to $0.1 \%$ m for the standard YIG. ${ }^{209}$ This material is known as the ferrite with the largest magneto-optical Faraday effect in the visible region of the optical spectrum. BIG is not thermodynamically stable, and for this reason $\mathrm{Bi}_{3} \mathrm{Fe}_{5} \mathrm{O}_{12}$ films cannot be prepared by LPE. Okuda et al. ${ }^{209}$ synthesized BIG by direct epitaxial growth via vapor phase using a reactive ion beam sputtering technique. The saturation magnetization of the resulting film was measured to be 1500 Gauss at room temperature while the uniaxial anisotropy energy $+7.2 \times 10^{4} \mathrm{erg} / \mathrm{cm}^{3}$.

In a somewhat parallel study, Kahl et al. ${ }^{204}$ studied the effect of deposition conditions on magnetic properties of $\mathrm{Y}_{3} \mathrm{Fe}_{5} \mathrm{O}_{12}$ and $\mathrm{Bi}_{3} \mathrm{Fe}_{5} \mathrm{O}_{12}$ films by pulsed laser deposition and showed that low laser repetition rates resulted in a reduction of saturation magnetization of YIG by a factor of 5. Small changes in the deposition conditions and lattice match for BIG films had a minor effect on the spectra of Faraday rotation but changed the magnetic coercivity by a factor of 10 . Coercivities of the thinner YIG films were higher than those of thicker films, and increased with lattice mismatch between the film and substrate. The wavelength at which the maximum Faraday rotation was observed for BIG films in the visible range of the optical spectrum depended on the film thickness. The wavelength redshifted by about $40 \mathrm{~nm}$ for a $2.6 \mu \mathrm{m}$ thick film as compared to a $470 \mathrm{~nm}$ thick film. 
Growth of Bi-substituted YIG films with a (111) orientation on the (111) plane of gadolinium gallium garnet (GGG) substrate by laser ablation has also been reported. ${ }^{204}$ Films had a garnet single phase with nearly constant composition in the temperature range between 490 and $580{ }^{\circ} \mathrm{C}$ which was near the target composition. The saturation magnetization of the film was 1500 Gauss at room temperature while the Faraday rotation angle $\theta_{F}$ at a wavelength of $830 \mathrm{~nm}$ was observed to be $-0.3 \times 10^{4} \% \mathrm{~cm}$. It was found earlier that an increase in the amount of Bi substituted for $\mathrm{Y}$ makes Faraday rotation angle change linearly at a rate of $-2.1 \times 10^{4} \% \mathrm{~cm} .{ }^{210}$

\subsection{Self Biasing in ferrites}

The applied magnetic bias field in microwave ferrite devices consists of two parts: the permanent part and the variable part. The permanent part is the sum of the external permanent magnet and the internal (uniaxial) anisotropy field of the substrate material, which provides constant magnetic bias. A variable bias field is then superimposed on the permanent part, resulting in local variation of the bias field near its permanent field value. The variable field is usually obtained by using a solenoid coil. The bias condition is usually devised near the ferromagnetic resonance (FMR) in which the permeability of the ferrite material is a sensitive function of the bias field. Consequently, a slight change in the bias field would result in a significant change in permeability. Reducing the bias current in the solenoid coil leads to enhanced switching speeds and lower power dissipations in e.g. a phase shifter. To reduce the bias current in the solenoid coil, the range of the variable bias field must be kept as a low as possible. This implies that the 
Özgür et al., submitted to Journal of Materials Science: Materials in Electronics, 2009 Microwave Ferrites, Part 1: Fundamental properties

permanent part of magnetic bias should be as high as possible. It should be noted that the price paid for this is that when FMR is approached, the ferrite material becomes very lossy, and the spin precessional motion experiences a significant magnetic damping torque. Another problem that microwave passive components face is their relatively large size because of the requisite permanent magnets which provide the necessary external magnetic biasing field. Having to rely on external permanent magnetic fields makes it difficult to reduce the device size and cost.

A promising approach to circumvent the above mentioned problems is the use of ferrite materials which exhibit high effective internal fields as a result of strong crystalline anisotropy. Such a self-biased material remains magnetized even after removing the external applied magnetic field, thus, eliminating the need for an external permanent magnet. To be successful, the magnetic materials must possess high saturation magnetization $\left(4 \pi M_{s}\right)$, high remanent magnetization $\left(M_{r}\right)$, and adjustable magnetic anisotropy fields $\left(H_{a}\right)$, and small resonance linewidths $\Delta H_{\mathrm{FMR}}$. And the larger the anisotropy field, the higher the ferrimagnetic resonance frequency for a fixed external field in accordance with Equation 78, Equation 83, and Equation 84 (e.g. $f_{r}=(2.8 \mathrm{MHz} / \mathrm{Oe})\left(H_{0}+H_{a}-4 \pi M_{s}\right)$ for a uniaxial thin film when the applied magnetic bias, $H_{0}$, perpendicular to the film plane). This is well illustrated in Figure 23 where the dependence of $f_{r}$ on $H_{0}$ is plotted for different $H_{a}$ values. It is seen that the $f_{r}\left(H_{0}\right)$ lines move up in frequency with increasing $H_{a}$, indicating higher operating frequencies. Most importantly, when no external magnetic field is applied $\left(H_{0}=0\right), f_{r}$ is distinct from zero at higher $H_{a}$ values due to self-bias induced by anisotropy. For example, for $H_{a}=17 \mathrm{kOe}$, 
Özgür et al., submitted to Journal of Materials Science: Materials in Electronics, 2009 Microwave Ferrites, Part 1: Fundamental properties

only internally biased $f_{r}\left(H_{0}=0\right)$ is $\sim 50 \mathrm{GHz}$ increasing to $80 \mathrm{GHz}$ with augmentation from $H_{0}$. As stated above, hexaferrites have strong uniaxial anisotropy up to $20 \mathrm{kOe}$ (see Table 8), depending on the kind of hexaferrite and preparation conditions. Such high values of uniaxial anisotropy allow tuning of the resonance frequency from $20-100 \mathrm{GHz}$ at relatively low magnetic biases. This degree of freedom afforded by large anisotropy makes this class of ferrites a choice material for high frequency passive microwave devices. In garnet and spinel ferrites, which have much smaller uniaxial anisotropy, to achieve such high frequencies one would have to apply huge magnetic fields. Consequently, YIG ferrites are usually used for microwave devices in the frequency range of $1-10 \mathrm{GHz}$.

\section{Summary and Outlook}

This review (Part 1) discusses the fundamentals of ferrite materials, interconnecting chemical, structural, and magnetic properties. Furthermore, various types of ferrites in use in microwave systems are treated. High resistivity (low rf loss) ferrimagnets find a plethora of applications in passive microwave components such as isolators, circulators, phase shifters, and miniature antennas operating in a wide range of frequencies. Frequency tuning has been traditionally obtained by externally applied magnetic fields with a permanent magnet or small electromagnet by passing current through coils. The applied magnetic bias field in microwave ferrite devices consists of a permanent part and a variable part, which is superimposed on the permanent part resulting in local variations of the bias field. The range of the variable bias field, which is usually generated by using 
Özgür et al., submitted to Journal of Materials Science: Materials in Electronics, 2009 Microwave Ferrites, Part 1: Fundamental properties

a solenoid coil, must be kept as a low as possible, to reduce the bias current in solenoids and therefore, to reduce the power dissipation and increase the switching speeds. This implies that the permanent part of magnetic bias, which is the sum of the external permanent magnet field and the internal (uniaxial) anisotropy field of the ferrite material, should be as high as possible for high frequency operation. However, a large external magnetic bias requires large permanent magnets resulting in relatively large size microwave passive components. Having to rely on external permanent magnetic fields makes it difficult to reduce the device size and cost. To circumvent this problem, a promising approach is to use ferrite materials with high effective internal fields, such as hexaferrites, courtesy of strong crystalline anisotropy. Such a self-biased material remains magnetized even after removing the external applied magnetic field, thus, eliminating the need for an external permanent magnet. This degree of freedom afforded by large anisotropy makes this class of ferrites a choice material for high frequency passive microwave devices. In garnet and spinel ferrites, however, the uniaxial anisotropy is much smaller, and to achieve such high frequency operation one would have to apply huge magnetic fields.

Part 2 of this review ${ }^{3}$ will discuss the basis for coupling between electrical and magnetic properties. These fundamental treatments will culminate in the discussion of microwave passive components, including the highly attractive electrical tuning of passive components by use of laminated systems combining piezoelectric materials, ideal for inducing mechanical strain by applied electric field efficiently, and magnetic materials 
Özgür et al., submitted to Journal of Materials Science: Materials in Electronics, 2009 Microwave Ferrites, Part 1: Fundamental properties

with large magnetostriction coefficients to induce a change in magnetic susceptibility due to strain.

\section{Acknowledgments}

The research at VCU is made possible by funds from the Office of Naval Research under direction of the program monitor, Dr. Ingham Mack and his predecessor, Dr. C. E. C. Wood. The authors would like to thank Professors Y.-K. Hong, J.-G. Yoon, C. Vittoria, and C. M. Srinivasan, and Dr. Cole Litton for useful discussions and in some cases manuscript and sample exchange, and graduate student E. Rowe for proofreading the manuscript and expanding the tables. 
Özgür et al., submitted to Journal of Materials Science: Materials in Electronics, 2009 Microwave Ferrites, Part 1: Fundamental properties

\section{FIGURE CAPTIONS}

Figure 1: The evolution of the figure of merit, maximum energy product which is the product of magnetic induction and maximum magnetic field (in terms of $\mathrm{kJm}^{-3}$ and also GaussOe). ii $^{\text {i }}$

Figure 2: The magnetocrystalline anisotropy in (a) cubic-like spinel structure (magnetite - $\mathrm{Fe}_{3} \mathrm{O}_{4}$ ), and (b) hexagonal structure such as Co with $H_{a}$ being the anisotropy field. In $\mathrm{Fe}_{3} \mathrm{O}_{4}$, the $<111>$ like and $<100>$ like directions represent the easy and hard directions, respectively. In Co, [0001] and [10 $\overline{1} 0]$-like directions represent the easy and hard directions, respectively.

Figure 3: Density of states vs. energy for the two spin components. In the non magnetic states (a), the occupancy by spin up and spin down electrons is the same. In Pauli magnetism at absolute zero when a magnetic field is applied, the spin down and spin up electrons are moved away from each other which leads to unparity in that the number of electrons with one type of spin would dominate over the other (b). The effect of the applied magnetic field is amplified to show the point. In ferromagnetic material and below the Curie temperature the effective field or the molecular field is so large, thus this splitting is sizeable and no external magnetic field is needed for the shift shown.

Figure 4: The well-known triangle used to describe the pathways between mechanical, electrical, and thermal energies in a class of non-centro-symmetric materials exhibiting piezoelectric effect, pyroelectric effect (converse of electrocaloric effect), piezocaloric 
Özgür et al., submitted to Journal of Materials Science: Materials in Electronics, 2009 Microwave Ferrites, Part 1: Fundamental properties

effect (converse of thermal expansion), pyromagnetic effect (converse of magnetocaloric effect), and magnetoelectric polarizability and so on. For simplicity, the magnetic property node is not shown. Tensor rank of the variable is shown in parentheses and the tensor rank of the property is shown in brackets. (Motivated by Nye. ${ }^{7}$ )

Figure 5: The well-known triangle used to describe the pathways between properties such as electric field, polarization, and magnetic field with connected properties such as strain $(\varepsilon)$, electrical polarization $(\mathrm{P})$, and magnetization $(\mathrm{M})$. Coupling coefficients between the various properties and external forces are also shown: electric susceptibility $\left(\chi_{E}\right)$, magnetic susceptibility $\left(\chi_{M}\right)$, piezoelectricity $\left(d_{E}\right)$ and magnetostriction $\left(d_{M}\right)$ tensors, and compliance tensor $(\mathrm{S}) . \alpha_{\mathrm{EM}}$ depicts the coupling between magnetic field and the induced electric polarization thus the electric field. For simplicity, the optical property node and its effect on the other properties or vice versa are not shown.

Figure 6: Hysteresis loop in the electric field vs. polarization curve in a ferroelectric material. Upon application of an electric field in the positive direction, the polarization increases and when all the domains are oriented, the polarization saturates. When the field strength is reduced the direction of the polarization remains until a specific reverse field, coercive field, which forces the domains to charge direction, is reached. As the field strength is increased further, the polarization again saturates. As the field intensity is reduced the net polarization remains in the same direction until a specific positive field, coercive field, which forces all the domains to switch polarization direction. The polarization remaining at zero field is called the remanent polarization. 
Özgür et al., submitted to Journal of Materials Science: Materials in Electronics, 2009 Microwave Ferrites, Part 1: Fundamental properties

Figure 7: Hysteresis loop in the magnetization vs. magnetic field curve in a ferromagnetic/ferrimagnetic material. Upon application of a magnetic field, the magnetization increases and when all the domains (magnetic dipoles) are oriented coherently, the magnetization saturates. When the magnetic field is reduced the magnetization does not reduce immediately. The direction is switched only after a specific reverse magnetic field, coercive field, is reached. As the field is increased further in the negative direction, the magnetization again saturates. As the magnetic field intensity is reduced the net polarization remains in the same direction until a specific positive field, coercive field, which forces all the domains to switch polarization direction, is reached. The magnetization remaining at zero field is called the remanent magnetization. The red solid hysteresis curve is for intrinsic magnetic polarization (intensity of magnetization) $J_{M}=\mu_{0} M=B-\mu_{0} H$. The black dashed curve is the sum of intrinsic and permeability curves together: $B=\mu_{0} H+J_{M}=\mu_{0} H+\mu_{0} M$. The maximum $\mathrm{BH}$ product $\left(\mathrm{kJ} / \mathrm{m}^{3}\right)$ indicated by the gray area represents the maximum energy storage per unit volume and is the figure of merit most often used for permanent magnet materials.

Figure 8: Schematic of an "octant" of a garnet crystal structure (lattice constant "a") showing only cation positions.

Figure 9: An "octant" of a garnet crystal structure (lattice constant "a") showing a trivalent ion of iron on a site surrounded by six oxygen ions in octahedral symmetry, a divalent ion of iron on a site surrounded by four oxygen ions in tetrahedral symmetry, 
Özgür et al., submitted to Journal of Materials Science: Materials in Electronics, 2009 Microwave Ferrites, Part 1: Fundamental properties

and a rare-earth ion surrounded by 8 oxygen ions which form an 8 -cornered 12-sided polyhedron. (After Geller and Gilleo. ${ }^{10}$ )

Figure 10: (a) Spinel unit cell structure, (b) octahedral interstice (B site: 32 per unit cell, 16 occupied), and (c) tetrahedral interstice (A site: 64 per unit cell, 8 occupied). In (a) the ionic positions are the same in octants sharing only one edge and different in octants sharing a face. Each octant contains 4 Oxygen ions. In (a) ionic positions in only two adjacent octants are shown, where the octant on the left contains octahedral and the one on the right contains tetrahedral sites. All ions are positioned on body diagonals of the octants and the octant on the right contains a tetrahedral site at the octant center.

Figure 11: Chemical composition diagram showing how the hexagonal ferrites are derived from the spinel $\mathrm{MeO} \cdot \mathrm{Fe}_{2} \mathrm{O}_{3}$ structure.

Figure 12: The schematic structure of the hexaferrite $\mathrm{BaFe}_{12} \mathrm{O}_{19}$. The arrows on Fe ions represent the direction of spin polarization. $2 a, 12 k$, and $4 f_{2}$ are octahedral, $4 f_{1}$ are tetrahedral, and $2 b$ are hexahedral (trigonal bipyramidal) sites. The unit cell contains a total of $38 \mathrm{O}^{2-}$ ions, $2 \mathrm{Ba}^{2+}$ ions, and $24 \mathrm{Fe}^{3+}$ ions. $\mathrm{Fe}^{3+}$ ions in $12 k, 2 a$, and $2 b$ sites (16 total per unit cell) have their spins up, while the $\mathrm{Fe}^{3+}$ ions in $4 f_{1}$ and $4 f_{2}$ sites ( 8 total per unit cell) have their spins down, which results in a net total of 8 spins up, and therefore, a total moment of $8 \times 5 \mu_{\mathrm{B}}=40 \mu_{\mathrm{B}}$ per unit cell that contains two $\mathrm{Ba}^{2+}$ ions. The $\mathrm{R}$ and $\mathrm{S}$ sub-units shown have chemical formulae $\mathrm{R}=\left(\mathrm{Ba}^{2+} \mathrm{Fe}_{6}^{3+} \mathrm{O}_{11}^{2-}\right)^{2-}$ and $\mathrm{S}=\left(\mathrm{Fe}_{6}^{3+} \mathrm{O}_{8}^{2-}\right)^{2+}$. The asterix $\left(^{*}\right)$ indicates that the corresponding sub-unit is rotated $180^{\circ}$ around the hexagonal axis. 
Özgür et al., submitted to Journal of Materials Science: Materials in Electronics, 2009 Microwave Ferrites, Part 1: Fundamental properties

Figure 13: A schematic of dipole moment $\mathbf{m}$ precessing about a static magnetic field $\mathbf{H}_{\mathbf{0}}$. When the frequency of the alternating magnetic field $\mathbf{H}_{\mathbf{a c}}$ (applied normal to the static field) is near the natural precession frequency the precession of the magnetic moment grows.

Figure 14: The real (dispersive) and the imaginary (dissipative) components of the susceptibility tensor elements computed for different damping factors ( $\phi=\gamma \mu_{0} \Delta H / 2 \omega_{0}=2 \phi H_{0}$ ) using a resonance frequency of $2.8 \mathrm{GHz}$ and a saturation magnetization of $4 \pi \mathrm{M}_{\mathrm{s}}=3000$ Gauss. The medium has been assumed to be spherical and magnetically saturated at low fields.

Figure 15: A pictorial overview of the magnetic relations processes that affect the FMR damping in ferrites. (After Schloemann. ${ }^{45}$ )

Figure 16:.FMR half power linewidth of hipped YIG sphere of $2.04 \mathrm{~mm}$ diameter. The solid squares show the data, the solid line is the calculated linewidth for the two-magnon anisotropy scattering process, and the dashed line shows the two-magnon response shifted upward by $0.03 f$ to account for the intrinsic linewidth of 0.3 Oe. (After Nazarov et $\left.a .^{49}\right)$

Figure 17: Effective linewidth for conventionally sintered (CS) and ultra dense (UD) YIG sphere samples measured outside the manifold region at a signal drive frequency of $10 \mathrm{GHz}$. (After Mo et al. ${ }^{53}$ ) 
Özgür et al., submitted to Journal of Materials Science: Materials in Electronics, 2009 Microwave Ferrites, Part 1: Fundamental properties

Figure 18: (a) Absorption derivative versus the applied static magnetic field at $60.3 \mathrm{GHz}$ for the main FMR mode in a PLD grown $0.85 \mu$ m-thick BaM film. The closed circles show the data and the solid line is a fit based on the a Lorentzian absorption response. (b) Half power linewidth for the same FMR mode as a function of the frequency. The data for bulk BaM from Karim et al. ${ }^{57}$ are shown with squares. The solid line is a linear fit to all the data. (After Song et $a .^{54}$ )

Figure 19: Saturation magnetization, $M_{s}$, and coercivity, $H_{c}$, of $\operatorname{Sr}\left[\mathrm{Zn}_{0.7} \mathrm{Nb}_{0.3}\right]_{x} \mathrm{Fe}_{12-x} \mathrm{O}_{19}$ as a function of $x$, for samples annealed at $950{ }^{\circ} \mathrm{C} / 5$ h. (After Fang et al. ${ }^{75}$ )

Figure 20: The magnetization curves parallel and perpendicular to the magnetic alignment direction used during sample preparation (surface normal) for a $\mathrm{BaZn}_{2-}$ ${ }_{x} \mathrm{Co}_{x} \mathrm{Fe}_{16} \mathrm{O}_{27}$ sample with $x=1.5$. The field corresponding to the intersection of the two curves is the anisotropy field $H_{a}$ for samples with $c$-axis anisotropy or $H_{\theta}$ for samples with $c$-plane anisotropy. Inset shows the anisotropy field $H_{a}$ (c-axis) or $H_{\theta}$ (c-plane) for various Co compositions, $x$. The values of anisotropy fields were determined based on two methods: the magnetization curves parallel and perpendicular to the magnetic alignment direction (open squares) and the law of approach to saturation magnetization (open circles). (After Li et al. $^{24}$ )

Figure 21: The variation of saturation magnetization $\left(M_{s}\right)$ with the average particle size (D nm) in $\mathrm{Y}_{3-x} \mathrm{Gd}_{x} \mathrm{Fe}_{5} \mathrm{O}_{12}$ prepared by sol-gel. (After Cheng et al. ${ }^{193}$ )

Figure 22: Spontaneous magnetization for yttrium and rare earth iron garnets as a function of temperature. (After Rodrigue. ${ }^{197}$ ) 
Özgür et al., submitted to Journal of Materials Science: Materials in Electronics, 2009 Microwave Ferrites, Part 1: Fundamental properties

Figure 23: Ferrite resonance frequency as a function of the applied field $H_{0}$ (a) for different values of anisotropy, $H_{a}$, in a spherical specimen and (b) for different values of saturation magnetization, $4 \pi M_{s}$, in a thin film. 
Özgür et al., submitted to Journal of Materials Science: Materials in Electronics, 2009 Microwave Ferrites, Part 1: Fundamental properties

\section{TABLES}


Table 1: The electronic configuration for the $d$ shell in transition metals, and the relevant spin, orbital, and total angular momentum along with the nomenclature used. The up and down arrows represent the spins up and down, respectively. Among the listed transition metals, $\mathrm{Sc}, \mathrm{Ti}, \mathrm{V}$, and $\mathrm{Mn}\left(3 \mathrm{~d}^{5}\right)$ are paramagnetic, $\mathrm{Cr}$ is antiferromagnetic, $\mathrm{Fe}, \mathrm{Co}$, and $\mathrm{Ni}$ are ferromagnetic, and $\mathrm{Cu}$ and $\mathrm{Zn}$ are diamagnetic at room temperature.

\begin{tabular}{|l|l|l|l|l|l|l|l|l|l|l|}
\hline element & $\begin{array}{l}d \text { shell } \\
\text { electrons } \\
(l=2) \\
\mathrm{n}\end{array}$ & $l_{\mathrm{z}}=-2$ & $l_{\mathrm{z}}=-1$ & $l_{\mathrm{z}}=0$ & $l_{\mathrm{z}}=+1$ & $l_{\mathrm{z}}=+2$ & $\begin{array}{l}\mathrm{S}= \\
(1 / 2)\left(\mathrm{n} \uparrow-\mathrm{n}_{\downarrow}\right)\end{array}$ & $L=\left|\sum l_{z}\right|$ & $\begin{array}{l}\mathrm{J}= \\
(\mathrm{L}-\mathrm{S} \uparrow),\left(\mathrm{L}+\mathrm{S}_{\downarrow}\right)\end{array}$ & $\begin{array}{l}\text { Symbol X in } \\
\text { the form } \\
2 \mathrm{~S}+1\end{array}$ \\
\hline $\mathrm{Sc}$ & 1 & $\uparrow$ & & & & & $1 / 2$ & 2 & $3 / 2$ & ${ }^{2} \mathrm{D}_{3 / 2}$ \\
\hline $\mathrm{Ti}$ & 2 & $\uparrow$ & $\uparrow$ & & & & 1 & 3 & 2 & ${ }^{3} \mathrm{~F}_{2}$ \\
\hline $\mathrm{V}$ & 3 & $\uparrow$ & $\uparrow$ & $\uparrow$ & & & $3 / 2$ & 3 & $3 / 2$ & ${ }^{4} \mathrm{~F}_{3 / 2}$ \\
\hline & 4 & $\uparrow$ & $\uparrow$ & $\uparrow$ & $\uparrow$ & & 2 & 2 & 0 & ${ }^{6} \mathrm{D}_{0}$ \\
\hline $\begin{array}{l}C r, \mathrm{Mn} \\
\left(3 \mathrm{~d}^{5}\right)\end{array}$ & 5 & $\uparrow$ & $\uparrow$ & $\uparrow$ & $\uparrow$ & $\uparrow$ & $5 / 2$ & 0 & $5 / 2$ & ${ }^{6} \mathrm{~S}_{5 / 2}$ \\
\hline $\begin{array}{l}\mathrm{Mn} \\
\left(3 \mathrm{~d}^{4}\right)\end{array}$ & 4 & $\uparrow$ & $\uparrow$ & $\uparrow$ & $\uparrow$ & & 2 & 2 & 0 & ${ }^{5} \mathrm{D}_{0}$ \\
\hline $\mathrm{Fe}$ & 6 & $\uparrow \downarrow$ & $\uparrow$ & $\uparrow$ & $\uparrow$ & $\uparrow$ & 2 & 2 & 4 & ${ }^{5} \mathrm{D}_{4}$ \\
\hline $\mathrm{Co}$ & 7 & $\uparrow \downarrow$ & $\uparrow \downarrow$ & $\uparrow$ & $\uparrow$ & $\uparrow$ & $3 / 2$ & 3 & $9 / 2$ & ${ }^{4} \mathrm{~F}_{9 / 2}$ \\
\hline $\mathrm{Ni}$ & 8 & $\uparrow \downarrow$ & $\uparrow \downarrow$ & $\uparrow \downarrow$ & $\uparrow$ & $\uparrow$ & 1 & 3 & 4 & ${ }^{3} \mathrm{~F}_{4}$ \\
\hline & 9 & $\uparrow \downarrow$ & $\uparrow \downarrow$ & $\uparrow \downarrow$ & $\uparrow \downarrow$ & $\uparrow$ & $1 / 2$ & 2 & $5 / 2$ & ${ }^{2} \mathrm{D}_{5 / 2}$ \\
\hline $\mathrm{Cu}, \mathrm{Zn}$ & 10 & $\uparrow \downarrow$ & $\uparrow \downarrow$ & $\uparrow \downarrow$ & $\uparrow \downarrow$ & $\uparrow \downarrow$ & 0 & 0 & 0 & ${ }^{1} \mathrm{~S}_{0}$ \\
\hline
\end{tabular}

$L=\left|\sum l_{z}\right|=\left|\left(l_{-2}\right)+\left(l_{-1}\right)+\left(l_{0}\right)+\left(l_{+1}\right)+\left(l_{+2}\right)\right|$

The label symbol, $\mathrm{X}$, is determined as follows: for $L=0,1,2,3,4,5,6, \mathrm{X}=\mathrm{S}, \mathrm{P}, \mathrm{D}, \mathrm{F}, \mathrm{G}, \mathrm{H}$, I, respectively. 
Özgür et al., submitted to Journal of Materials Science: Materials in Electronics, 2009

Microwave Ferrites, Part 1: Fundamental properties 
Table 2: Classification of materials based on the amplitude of their magnetic $\begin{array}{lllll}\text { susceptibility, } & \chi & \text { In } & \text { part } & \text { after }\end{array}$

http://www.magnets.bham.ac.uk/magnetic_materials/type.htm.

\begin{tabular}{|c|c|c|c|c|}
\hline $\begin{array}{c}\text { Type of } \\
\text { magnetism }\end{array}$ & $\begin{array}{c}\text { Magnetic } \\
\text { susceptibility, } \chi\end{array}$ & Atomic / Magnetic Behavior & $\begin{array}{c}\text { Temperature } \\
\text { dependence }\end{array}$ & Examples and comments \\
\hline Diamagnetic & $\begin{array}{l}\text { Negative and } \\
\text { small, } \\
\text { Au: }-2.74 \times 10^{-6} \\
\mathrm{Cu}:-0.77 \times 10^{-6}\end{array}$ & $\left.00000\right|^{M}$ & $\begin{array}{l}\text { Temperature } \\
\text { independent }\end{array}$ & $\begin{array}{l}\text { The shells of the atoms are closed } \\
\text { as in the case of covalent solids } \\
\text { such as } \mathrm{Ge}, \mathrm{Si} \text {, and metals such as } \\
\mathrm{Au}, \mathrm{Cu}, \mathrm{Ag} \text {, etc. }\end{array}$ \\
\hline \multirow{2}{*}{ Paramagnetic } & $\begin{array}{l}\text { Positive and } \\
\text { small, } \\
\beta-S n: 0.19 \times 10^{-6} \\
\text { Pt: } 21.04 \times 10^{-6} \\
\text { Mn: } 66.10 \times 10^{-6} \\
10^{-5}-10^{-4} \\
\end{array}$ & \multirow[t]{2}{*}{$\downarrow \rightarrow 1+4<$} & $\begin{array}{l}\text { Temperature } \\
\text { independent }\end{array}$ & $\begin{array}{l}\text { Atoms have randomly oriented } \\
\text { magnetic moments as in alkali and } \\
\text { transition metals }\end{array}$ \\
\hline & $\begin{array}{l}\text { Positive and } \\
\text { small }\end{array}$ & & $\begin{array}{l}\text { Follows Curie or } \\
\text { Curie-Weiss law: } \\
\qquad \chi=\frac{C}{T-\theta}\end{array}$ & $\begin{array}{l}\text { Atoms constituting the material } \\
\text { have a permanent magnetic } \\
\text { moment as in ferromagnets }(\mathrm{Fe}) \text {, } \\
\text { antiferromagnets }(\mathrm{Cr}) \text {, } \\
\text { ferrimagnets }\left(\mathrm{Fe}_{2} \mathrm{O}_{3}\right) \text { at high } \\
\text { temperatures }\end{array}$ \\
\hline Ferromagnetic & $\begin{array}{l}\text { Positive and } \\
\text { large, function } \\
\text { of applied field, } \\
\text { microstructure } \\
\text { dependent } \\
\text { Fe: } \sim 100,000\end{array}$ & $\uparrow \uparrow \uparrow$ & $\begin{array}{l}\text { Ferromagnetic } \\
\text { below Curie } \\
\text { temperature and } \\
\text { paramagnetic } \\
\text { above it }\end{array}$ & $\begin{array}{l}\text { Atoms have parallel aligned } \\
\text { magnetic moments, possesses } \\
\text { large permanent magnetization } \\
\text { even without external magnetic } \\
\text { field as in some transition metals } \\
\text { and rare earths such as } \mathrm{Fe}, \mathrm{Co}, \mathrm{Ni} \text {, } \\
\text { Gd, Dy }\end{array}$ \\
\hline Antiferromagnetic & $\begin{array}{l}\text { Positive and } \\
\text { small, } \\
\text { Cr: } 3.6 \times 10^{-6}\end{array}$ & 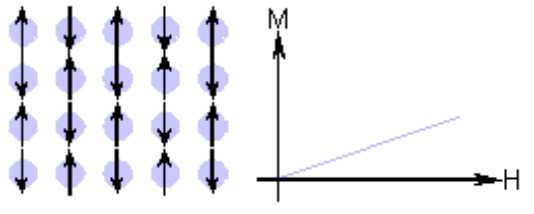 & $\begin{array}{l}\text { Antiferromagnetic } \\
\text { below the Néel } \\
\text { temperature and } \\
\text { paramagnetic } \\
\text { above it }\end{array}$ & $\begin{array}{l}\text { Atoms have mixed parallel and } \\
\text { anti-parallel aligned magnetic } \\
\text { moments Primarily oxides and } \\
\text { salts of transition metals such as } \\
\mathrm{MnO}, \mathrm{NiO}, \mathrm{MnF}_{2} \text {. }\end{array}$ \\
\hline Ferrimagnetic & $\begin{array}{l}\text { Positive and } \\
\text { large, function } \\
\text { of applied field, } \\
\text { microstructure } \\
\text { dependent, } \\
\text { Ba ferrite: } \sim 3\end{array}$ & 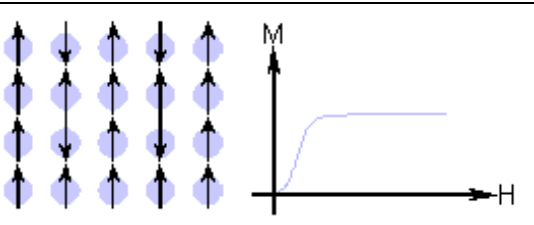 & $\begin{array}{l}\text { Ferrimagnetic } \\
\text { below the Curie } \\
\text { temperature and } \\
\text { paramagnetic } \\
\text { above it }\end{array}$ & $\begin{array}{l}\text { Atoms have anti-parallel aligned } \\
\text { magnetic moments, possesses } \\
\text { large magnetization even without } \\
\text { external magnetic field }\end{array}$ \\
\hline
\end{tabular}


Özgür et al., submitted to Journal of Materials Science: Materials in Electronics, 2009 Microwave Ferrites, Part 1: Fundamental properties

Table 3: The mass normalized saturation magnetization $\left(\sigma_{s}\right)$ and Néel temperature $\left(T_{N}\right)$ of a range of magnetic materials.

\begin{tabular}{|c|c|c|c|c|}
\hline Mineral & Composition & $\begin{array}{l}\text { Magnetic } \\
\text { Order }\end{array}$ & $\begin{array}{l}T_{N} \\
\left({ }^{\circ} \mathrm{C}\right)\end{array}$ & $\begin{array}{l}\sigma_{s} \\
\left(\mathrm{Am}^{2} / \mathrm{kg}\right)\end{array}$ \\
\hline \multicolumn{5}{|l|}{ Oxides } \\
\hline Magnetite & $\mathrm{Fe}_{3} \mathrm{O}_{4}$ & ferrimagnetic & \begin{tabular}{|l}
$575-$ \\
585 \\
\end{tabular} & $90-92$ \\
\hline Ulvospinel & $\mathrm{Fe}_{2} \mathrm{TiO}_{2}$ & AFM & -153 & \\
\hline Hematite & $\alpha \mathrm{Fe}_{2} \mathrm{O}_{3}$ & canted AFM & 675 & 0.4 \\
\hline Ilmenite & $\mathrm{FeTiO}_{2}$ & AFM & -233 & \\
\hline Maghemite & $\gamma \mathrm{Fe}_{2} \mathrm{O}_{3}$ & ferrimagnetic & $\sim 600$ & $\sim 80$ \\
\hline Jacobsite & $\mathrm{MnFe}_{2} \mathrm{O}_{4}$ & ferrimagnetic & 300 & 77 \\
\hline Trevorite & $\mathrm{NiFe}_{2} \mathrm{O}_{4}$ & ferrimagnetic & 585 & 51 \\
\hline Magnesioferrite & $\mathrm{MgFe}_{2} \mathrm{O}_{4}$ & ferrimagnetic & 440 & 21 \\
\hline Ba-hexaferrite & $\mathrm{BaFe}_{12} \mathrm{O}_{19}$ & ferrimagnetic & 450 & 75 \\
\hline Sr-hexaferrite & $\mathrm{SrFe}_{12} \mathrm{O}_{19}$ & ferrimagnetic & 450 & 75 \\
\hline \multicolumn{5}{|l|}{ Sulfides } \\
\hline Pyrrhotite & $\mathrm{Fe}_{7} \mathrm{~S}_{8}$ & ferrimagnetic & 320 & $\sim 20$ \\
\hline Greigite & $\mathrm{Fe}_{3} \mathrm{~S}_{4}$ & ferrimagnetic & $\sim 333$ & $\sim 25$ \\
\hline Troilite & $\mathrm{FeS}$ & AFM & 305 & \\
\hline \multicolumn{5}{|l|}{ Oxyhydroxides } \\
\hline Goethite & $\alpha \mathrm{FeOOH}$ & $\begin{array}{l}\text { AFM, weak } \\
\text { FM }\end{array}$ & $\sim 120$ & $<1$ \\
\hline Lepidocrocite & $\gamma \mathrm{FeOOH}$ & AFM & -196 & \\
\hline Feroxyhyte & $\delta \mathrm{FeOOH}$ & ferrimagnetic & $\sim 180$ & $<10$ \\
\hline \multicolumn{5}{|l|}{ Garnets } \\
\hline YIG & $\mathrm{Y}_{3} \mathrm{Fe}_{5} \mathrm{O}_{12}$ & ferrimagnetic & $\sim 290$ & 25 \\
\hline SmIG & $\mathrm{Sm}_{3} \mathrm{Fe}_{5} \mathrm{O}_{12}$ & ferrimagnetic & $\sim 290$ & 19 \\
\hline GdIG & $\mathrm{Gd}_{3} \mathrm{Fe}_{5} \mathrm{O}_{12}$ & ferrimagnetic & $\sim 290$ & 1.5 \\
\hline HoIG & $\mathrm{Ho}_{3} \mathrm{Fe}_{5} \mathrm{O}_{12}$ & ferrimagnetic & $\sim 305$ & 9 \\
\hline \multicolumn{5}{|l|}{ Metals \& Alloys } \\
\hline Iron & $\mathrm{Fe}$ & FM & 770 & 197 \\
\hline
\end{tabular}


Özgür et al., submitted to Journal of Materials Science: Materials in Electronics, 2009 Microwave Ferrites, Part 1: Fundamental properties

\begin{tabular}{|l|l|l|l|l|}
\hline Nickel & $\mathrm{Ni}$ & FM & 358 & 55 \\
\hline Cobalt & $\mathrm{Co}$ & FM & 1131 & 161 \\
\hline Awaruite & $\mathrm{Ni}_{3} \mathrm{Fe}$ & FM & 620 & 120 \\
\hline Wairauite & $\mathrm{CoFe}$ & FM & 986 & 235 \\
\hline & $\mathrm{SmCo}_{5}$ & FM & 720 & 109 \\
\hline & $\mathrm{Sm}_{2} \mathrm{Co}_{17}$ & FM & 820 & 118 \\
\hline
\end{tabular}

$\mathrm{FM}=$ ferromagnetic order

$\mathrm{AFM}=$ antiferromagnetic order

$T_{N}=$ Néel temperature (Curie Temperature, $T_{C}$, for ferromagnetic materials)

$\sigma_{s}=$ saturation magnetization at room-temperature (in mass-normalized SI units, i.e.

magnetization normalized to mass density) 
Özgür et al., submitted to Journal of Materials Science: Materials in Electronics, 2009 Microwave Ferrites, Part 1: Fundamental properties

Table 4: The relationship of some magnetic parameters in cgs and S.I. units. ${ }^{\text {ii }}$

\begin{tabular}{|c|c|c|c|}
\hline Quantity & $\begin{array}{c}\text { Gaussian } \\
\text { (cgs units) }^{*}\end{array}$ & S.I. Units ${ }^{\dagger}$ & $\begin{array}{l}\text { Conversion factor } \\
\text { (from cgs to S.I.) }\end{array}$ \\
\hline $\begin{array}{l}B \text {-field } \\
\text { (aliases Magnetic Induction, } \\
\text { Magnetic Flux density }\end{array}$ & Gauss & $\mathrm{T}$ & $10^{-4}$ \\
\hline $\begin{array}{l}H \text {-field } \\
\text { (aliases Magnetic field } \\
\text { strength/intensity, Applied Field) }\end{array}$ & Oe & $\mathrm{Am}^{-1}$ & $10^{3} / 4 \pi$ \\
\hline Magnetic moment $(\mathrm{m})$ & $\mathrm{emu}^{\#}$ & $\mathrm{Am}^{2}$ & $10^{-3}$ \\
\hline Magnetization $(M)$ & emu $\mathrm{cm}^{-3}$ & $\mathrm{Am}^{-1}$ & $10^{3}$ \\
\hline Magnetization $(4 \pi M)$ & Gauss & $\mathrm{Am}^{-1}$ & $10^{3} / 4 \pi$ \\
\hline Permeability $(\mu)$ & Dimensionless & $\mathrm{H} \mathrm{m}^{-1}$ & $4 \pi \times 10^{-7}$ \\
\hline Relative Permeability $\left(\mu_{r}\right)$ & - & Dimensionless & - \\
\hline Susceptibility $(\chi)$ & emu $\mathrm{cm}^{-3}$ & Dimensionless & $4 \pi$ \\
\hline $\begin{array}{l}\text { Maximum Energy Product } \\
\left(B H_{\max }\right)\end{array}$ & Gauss Oe & $\mathrm{k} \mathrm{J} \mathrm{m}{ }^{-3}$ & $10^{2} / 4 \pi$ \\
\hline Demagnetization factors $(N)$ & Dimensionless & Dimensionless & $1 / 4 \pi$ \\
\hline
\end{tabular}

${ }^{*}$ The cgs system of units is based on the relationship $\mathbf{B}=\mathbf{H}+4 \pi \mathbf{M}$

$\uparrow$ The S.I. system of units is based on the relationship $\mathbf{B}=\mu_{0}(\mathbf{H}+\mathbf{M})$

\# Note that emu is not a unit but an indicator of the unit system used: "electromagnetic units". 
Özgür et al., submitted to Journal of Materials Science: Materials in Electronics, 2009 Microwave Ferrites, Part 1: Fundamental properties

Table 5: Chemical composition and interrelation of hexagonal ferrites. (After Refs. 15 and 16)

\begin{tabular}{|c|c|c|c|}
\hline $\begin{array}{c}\text { Hexaferrite } \\
\text { type }\end{array}$ & Composition (chemical formula) & Stacking order & Interrelation \\
\hline $\mathrm{S}$ - spinel & $2 \mathrm{Me}^{2+} \cdot 2 \mathrm{Fe}_{2} \mathrm{O}_{4}$ or $2 \mathrm{MeO} \cdot 2 \mathrm{Fe}_{2} \mathrm{O}_{3}$ & & $\mathrm{~S}$ \\
\hline $\mathrm{M}$ & $\mathrm{Ba}^{2+} \mathrm{Fe}_{12} \mathrm{O}_{19}$ or $\mathrm{BaO} \cdot 6 \mathrm{Fe}_{2} \mathrm{O}_{3}$ & $\mathrm{RSR} * \mathrm{~S} *$ & $\mathrm{M}$ \\
\hline $\mathrm{W}$ & $\mathrm{Ba}^{2+} \mathrm{Me}_{2}{ }^{2+} \mathrm{Fe}_{16} \mathrm{O}_{27}$ or $\mathrm{BaO} \cdot 2 \mathrm{MeO} \cdot 8 \mathrm{Fe}_{2} \mathrm{O}_{3}$ & $\mathrm{RSSR} * \mathrm{~S} * \mathrm{~S} *$ & $\mathrm{M}+\mathrm{S}$ \\
\hline $\mathrm{Y}$ & $\mathrm{Ba}_{2}{ }^{2+} \mathrm{Me}_{2}{ }^{2+} \mathrm{Fe}_{12} \mathrm{O}_{22}$ or $2 \mathrm{BaO} \cdot 2 \mathrm{MeO} \cdot 6 \mathrm{Fe}_{2} \mathrm{O}_{3}$ & $\mathrm{TSTSTS}$ & $\mathrm{Y}$ \\
\hline $\mathrm{Z}$ & $\mathrm{Ba}_{3}{ }^{2+} \mathrm{Me}_{2}{ }^{2+} \mathrm{Fe}_{24} \mathrm{O}_{41}$ or $3 \mathrm{BaO} \cdot 2 \mathrm{MeO} \cdot 12 \mathrm{Fe}_{2} \mathrm{O}_{3}$ & $\mathrm{RSTSR} * \mathrm{~S} * \mathrm{~T} * \mathrm{~S} *$ & $\mathrm{M}+\mathrm{Y}$ \\
\hline $\mathrm{U}$ & $\mathrm{Ba}_{4}{ }^{2+} \mathrm{Me}_{2}{ }^{2+} \mathrm{Fe}_{36} \mathrm{O}_{60}$ or $4 \mathrm{BaO} \cdot 2 \mathrm{MeO} \cdot 18 \mathrm{Fe}_{2} \mathrm{O}_{3}$ & $\mathrm{RSR} * \mathrm{~S} * \mathrm{~T} * \mathrm{~S} *$ & $2 \mathrm{M}+\mathrm{Y}$ \\
\hline $\mathrm{X}$ & $\mathrm{Ba}_{2}{ }^{2+} \mathrm{Me}_{2}{ }^{2+} \mathrm{Fe}_{28} \mathrm{O}_{46}$ or $2 \mathrm{BaO} \cdot 2 \mathrm{MeO} \cdot 14 \mathrm{Fe}_{2} \mathrm{O}_{3}$ & $\mathrm{RSR} * \mathrm{~S} * \mathrm{~S} *$ & $2 \mathrm{M}+\mathrm{S}$ \\
\hline
\end{tabular}

\# Sub-units for stacking order, using $\mathrm{Me}=\mathrm{Fe}: \mathrm{S}=\mathrm{Fe}_{6} \mathrm{O}_{8}$ (spinel), $\mathrm{R}=\mathrm{BaFe}_{6} \mathrm{O}_{11}$ (hexagonal), and $\mathrm{T}=\mathrm{Ba}_{2} \mathrm{Fe}_{8} \mathrm{O}_{14}$ (hexagonal). The asterix (*) indicates that the corresponding sub-unit is rotated $180^{\circ}$ around the hexagonal axis. 
Özgür et al., submitted to Journal of Materials Science: Materials in Electronics, 2009 Microwave Ferrites, Part 1: Fundamental properties

Table 6: Room temperature nominal values for the saturation magnetization and the anisotropy field for various materials. (After Reference 32)

\begin{tabular}{|l|c|c|}
\hline \multicolumn{1}{|c|}{ Ferrite } & $4 \pi \mathrm{M}_{\mathrm{s}}$ (Gauss) & $\mathrm{H}_{\mathrm{a}}=2 \mathrm{~K}_{1} / \mathrm{M}_{\mathrm{s}}(\mathrm{Oe})$ \\
\hline $\mathrm{Y}_{3} \mathrm{Fe}_{5} \mathrm{O}_{12}(\mathrm{YIG})^{41}$ & 1750 & 82 \\
\hline $\mathrm{MgFe}_{2} \mathrm{O}_{4}$ & 1400 & -90 \\
\hline $\mathrm{MnFe}_{2} \mathrm{O}_{4}$ & 5200 & -140 \\
\hline $\mathrm{NiFe}_{2} \mathrm{O}_{4}$ & 3300 & -425 \\
\hline $\mathrm{NiFe}_{1.35} \mathrm{Al}_{0.65} \mathrm{O}_{4}$ & 465 & -2400 \\
\hline $\mathrm{Ni}_{0.9} \mathrm{Zn}_{0.1} \mathrm{Fe}_{2} \mathrm{O}_{4}$ & 4900 & -240 \\
\hline $\mathrm{FeFe}_{2} \mathrm{O}_{4}$ & 6000 & -460 \\
\hline $\mathrm{CuFe}_{2} \mathrm{O}_{4}$ & 1700 & -220 \\
\hline $\mathrm{Ni}_{0.932} \mathrm{Co}_{0.068} \mathrm{Fe}_{2} \mathrm{O}_{4}$ & 3475 & +1000 \\
\hline $\mathrm{CoFe}_{2} \mathrm{O}_{4}{ }_{42}$ & 5370 & 6800 \\
\hline $\mathrm{BaFe}_{12} \mathrm{O}_{19}(\mathrm{M}-\mathrm{type})$ & 4800 & 17000 \\
\hline $\mathrm{BaFe}_{18} \mathrm{O}_{27}\left(\mathrm{Fe}_{2} \mathrm{~W}\right)$ & 4000 & 19000 \\
\hline $\mathrm{Ba}_{2} \mathrm{Co}_{2} \mathrm{Fe}_{12} \mathrm{O}_{22}\left(\mathrm{Co}{ }_{2} \mathrm{Y}\right)$ & 2300 & 28000 \\
\hline
\end{tabular}


Table 7: Properties of some polycrystalline ferrite materials from Trans-Tech, Inc.iii

\begin{tabular}{|c|c|c|c|c|c|}
\hline Ferrite & $\begin{array}{c}\text { Saturation } \\
\text { magnetization, } \\
4 \pi M_{s} \text { (Gauss) }\end{array}$ & $\begin{array}{c}\text { FMR linewidth @3dB, } \\
\Delta \mathrm{H}(\mathrm{Oe})\end{array}$ & $\begin{array}{c}\text { Dielectric constant, } \\
\varepsilon_{r}\end{array}$ & Landé g-factor & $\tan \delta$ \\
\hline $\begin{array}{l}\text { Yttrium garnet } \\
\text { G-113 }\end{array}$ & 1780 & 25 & 15 & 1.97 & 0.0002 \\
\hline $\begin{array}{l}\text { Aluminum garnet } \\
\text { G-610 }\end{array}$ & 680 & 25 & 14.5 & 2 & 0.002 \\
\hline $\begin{array}{l}\text { Magnesium ferrite } \\
\text { TT1-390 }\end{array}$ & 2150 & 648 & 12.7 & 2.04 & 0.00025 \\
\hline $\begin{array}{l}\text { Nickel ferrite } \\
\text { TT2-101 }\end{array}$ & 3000 & 375 & 13 & 2.19 & 0.0025 \\
\hline $\begin{array}{l}\text { Lithium ferrite } \\
\text { TT71-4800 }\end{array}$ & 4800 & 240 & 14.5 & 2.01 & 0.0015 \\
\hline
\end{tabular}


Özgür et al., submitted to Journal of Materials Science: Materials in Electronics, 2009 Microwave Ferrites, Part 1: Fundamental properties

Table 8: Crystal and physical properties of some ferrites.

\begin{tabular}{|c|c|c|c|c|c|c|c|}
\hline & Material & Abbreviation & $T_{N}\left({ }^{\circ} \mathrm{C}\right)$ & $\begin{array}{l}\text { Landé } \\
\text { g-factor }\end{array}$ & $\begin{array}{c}4 \pi M_{s} \\
\text { (Gauss) }\end{array}$ & $\begin{array}{c}\text { Magnetic } \\
\text { anisotropy } \\
H_{a}(\mathrm{Oe})\end{array}$ & $\begin{array}{c}\text { Coercive } \\
\text { field } \\
H_{c}(\mathrm{Oe})\end{array}$ \\
\hline \multirow{9}{*}{ 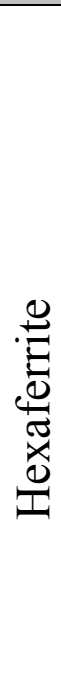 } & $\mathrm{BaFe}_{12} \mathrm{O}_{19}$ & $\mathrm{BaM}$ & 450 [17] & 1.87 [59] & 4000 [18] & 17460 [19] & 1935 [18] \\
\hline & $\mathrm{SrFe}_{12} \mathrm{O}_{19}$ & SrM & $455[20]$ & & $4320[62]$ & 16000 [20] & 6635 [21] \\
\hline & $\mathrm{Ba}_{4} \mathrm{Zn}_{2} \mathrm{Fe}_{36} \mathrm{O}_{60}$ & $\mathrm{Zn}_{2} \mathrm{U}$ & 400 & & $4223[63]$ & 10038 [63] & 2580 [64] \\
\hline & $\mathrm{Ba}_{2} \mathrm{Co}_{2} \mathrm{Fe}_{28} \mathrm{O}_{46}$ & $\mathrm{Co}_{2} \mathrm{X}$ & $467[22]$ & & $\begin{array}{c}3400 \\
{[22,65]}\end{array}$ & 9500 [22] & $50[22]$ \\
\hline & $\mathrm{Ba}_{2} \mathrm{MnZnFe}_{12} \mathrm{O}_{22}$ & MnZnY & $100[66]$ & & $2300[23]$ & 9500 [23] & $60[23]$ \\
\hline & $\mathrm{BaZn}_{2} \mathrm{Fe}_{16} \mathrm{O}_{27}$ & $\mathrm{Zn}_{2} \mathrm{~W}$ & & & 4950 [24] & 12000 [24] & 184 [24] \\
\hline & $\mathrm{BaCo}_{2} \mathrm{Fe}_{16} \mathrm{O}_{27}$ & $\mathrm{Co}_{2} \mathrm{~W}$ & 430 [59] & 1.90 [59] & $4800[24]$ & $21000[24]$ & $80[24]$ \\
\hline & $\mathrm{Ba}_{3} \mathrm{Co}_{2} \mathrm{Fe}_{24} \mathrm{O}_{41}$ & $\mathrm{Co}_{2} \mathrm{Z}$ & $400[58]$ & & $3300[25]$ & $12000[25]$ & $23[25]$ \\
\hline & $\mathrm{Ba}_{3} \mathrm{Zn}_{2} \mathrm{Fe}_{24} \mathrm{O}_{41}$ & $\mathrm{Zn}_{2} \mathrm{Z}$ & & & 3900 & 4810 & \\
\hline \multirow{5}{*}{$\begin{array}{l}\bar{\Xi} \\
\text { क } \\
\text { क }\end{array}$} & $\mathrm{MgFe}_{2} \mathrm{O}_{4}$ & $\mathrm{MgFO}$ & 320 [iii] & $2.06[59]$ & 2150 [iii] & $173[67]$ & 1.8 [iii] \\
\hline & $\mathrm{MnFe}_{2} \mathrm{O}_{4}$ & $\mathrm{MnFO}$ & $340[68]$ & $2.20[59]$ & $4000[26]$ & $5400[26]$ & $196[68]$ \\
\hline & $\mathrm{Li}_{0.2} \mathrm{Zn}_{0.6} \mathrm{Fe}_{2.2} \mathrm{O}_{4}$ & & 277 [59] & $2.08[59]$ & 2730 [59] & 7330 [59] & \\
\hline & $\mathrm{CoFe}_{2} \mathrm{O}_{4}$ & CFO & 520 & & $5370[42]$ & 6800 [42] & $\begin{array}{l}1566[27] \\
2720[28]\end{array}$ \\
\hline & $\mathrm{NiFe}_{2} \mathrm{O}_{4}$ & NFO & 585 [iii] & 2.4 [59] & 3000 [iii] & 425 & 5.7 [iii] \\
\hline \multirow{2}{*}{ } & $\mathrm{Y}_{3} \mathrm{Fe}_{5} \mathrm{O}_{12}$ & YIG & $280[17]$ & 2.00 [59] & $1750[41]$ & $82[41]$ & \\
\hline & $\mathrm{Y}_{3} \mathrm{Fe}_{5-\mathrm{x}} \mathrm{Ga}_{\mathrm{x}} \mathrm{O}_{12}$ & GaYIG & 167 & 2.03 [59] & 400 & & \\
\hline
\end{tabular}


Özgür et al., submitted to Journal of Materials Science: Materials in Electronics, 2009 Microwave Ferrites, Part 1: Fundamental properties

Ü. Özgür et al.

Journal of Materials Science: Materials in Electronics

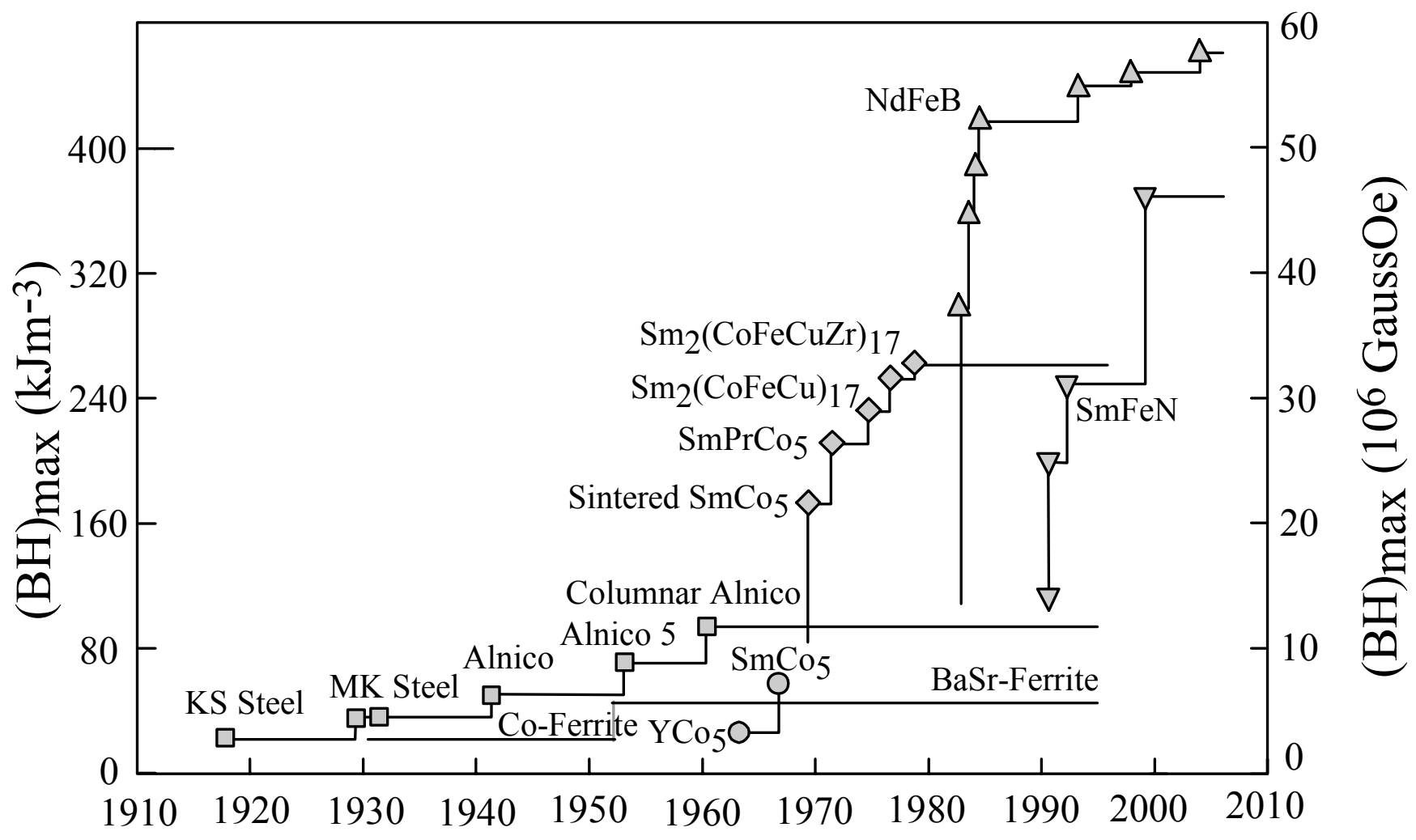

Figure 24

The evolution of the figure of merit, maximum energy product which is the product of magnetic induction and maximum magnetic field (in terms of $\mathrm{kJm}^{-3}$ and also GaussOe). 
Özgür et al., submitted to Journal of Materials Science: Materials in Electronics, 2009 Microwave Ferrites, Part 1: Fundamental properties

Ü. Özgür et al.

Journal of Materials Science: Materials in Electronics

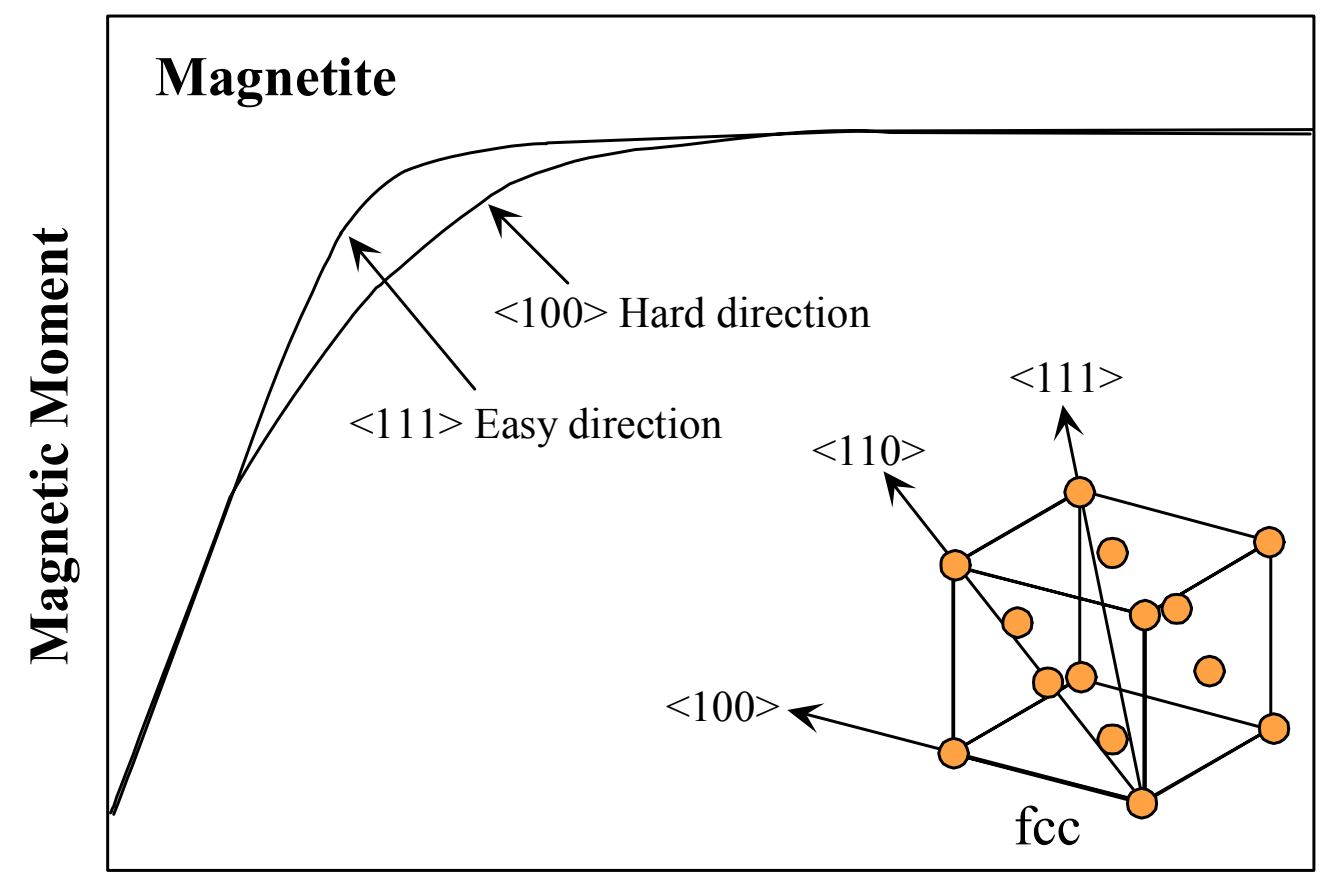

(a)

Applied field, $\mathbf{H}$

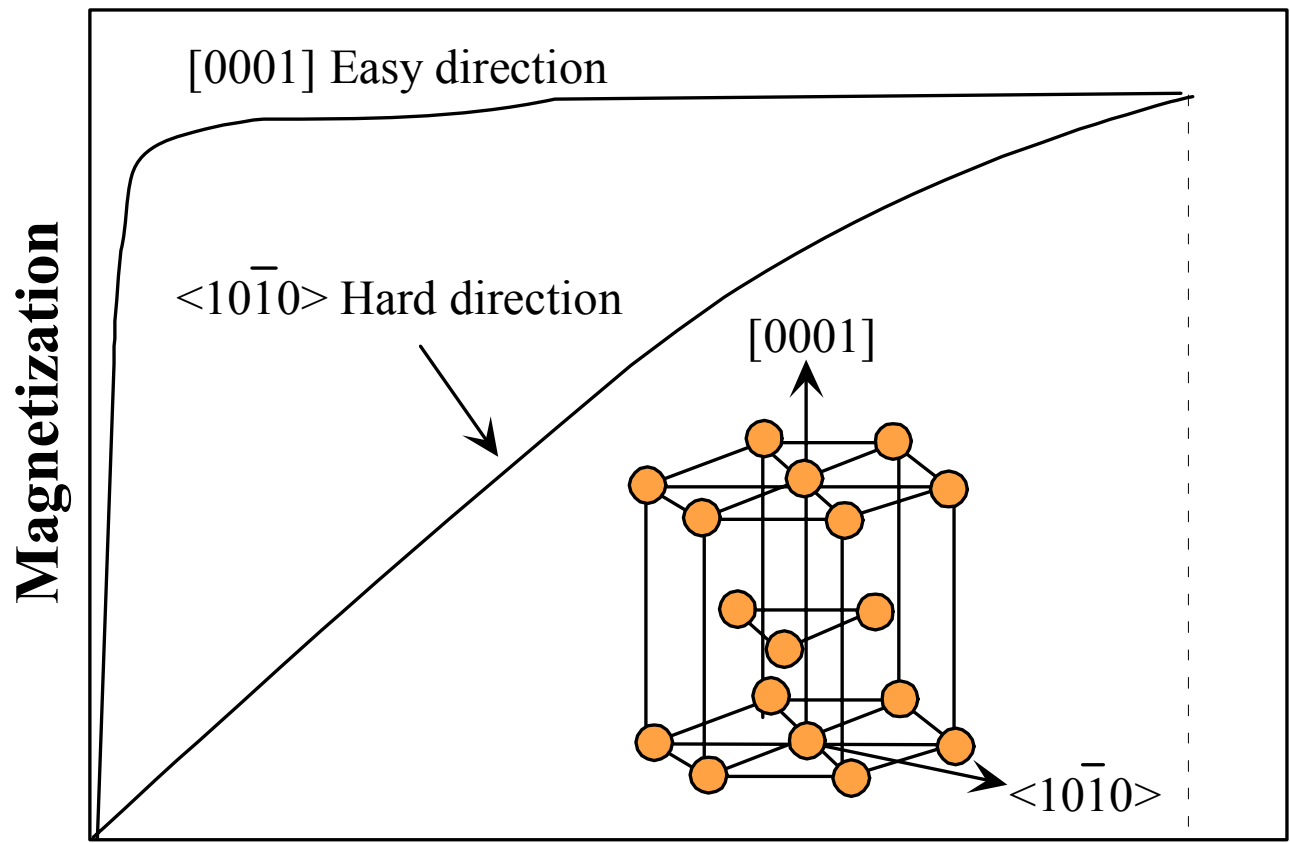

(b) 
Özgür et al., submitted to Journal of Materials Science: Materials in Electronics, 2009 Microwave Ferrites, Part 1: Fundamental properties

\section{Figure 25}

The magnetocrystalline anisotropy in (a) cubic-like spinel structure (magnetite- $\mathrm{Fe}_{3} \mathrm{O}_{4}$ ),

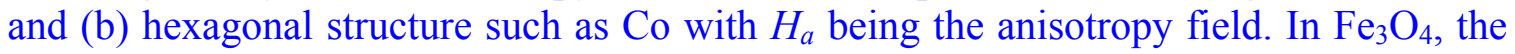
$<111>$ like and $<100>$ like directions represent the easy and hard directions, respectively. In $\mathrm{Co},[0001]$ and $\left[\begin{array}{lll}10 & 1 & 0\end{array}\right]$-like directions represent the easy and hard directions, respectively. 
Özgür et al., submitted to Journal of Materials Science: Materials in Electronics, 2009 Microwave Ferrites, Part 1: Fundamental properties

Ü. Özgür et al.

Journal of Materials Science: Materials in Electronics

\section{no magnetic field}

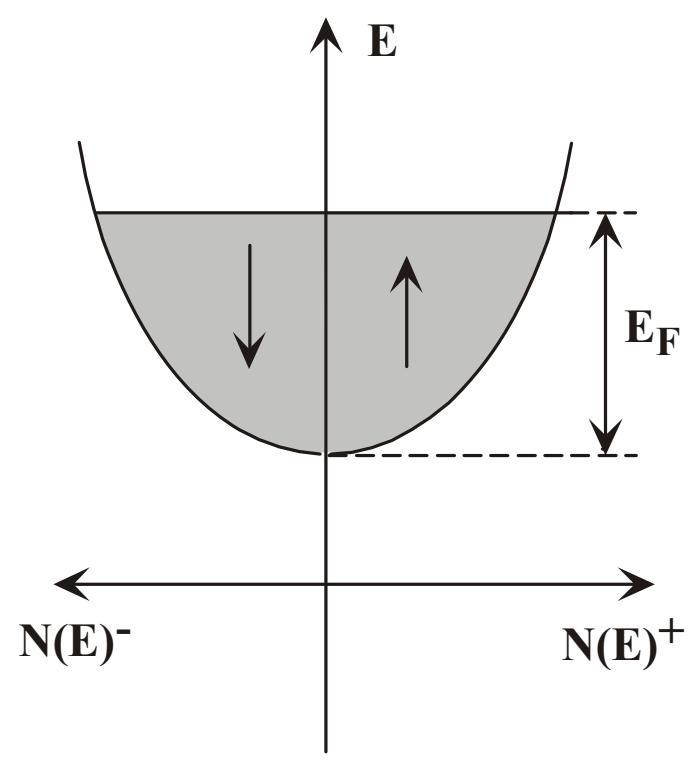

(a) magnetic field, $B$

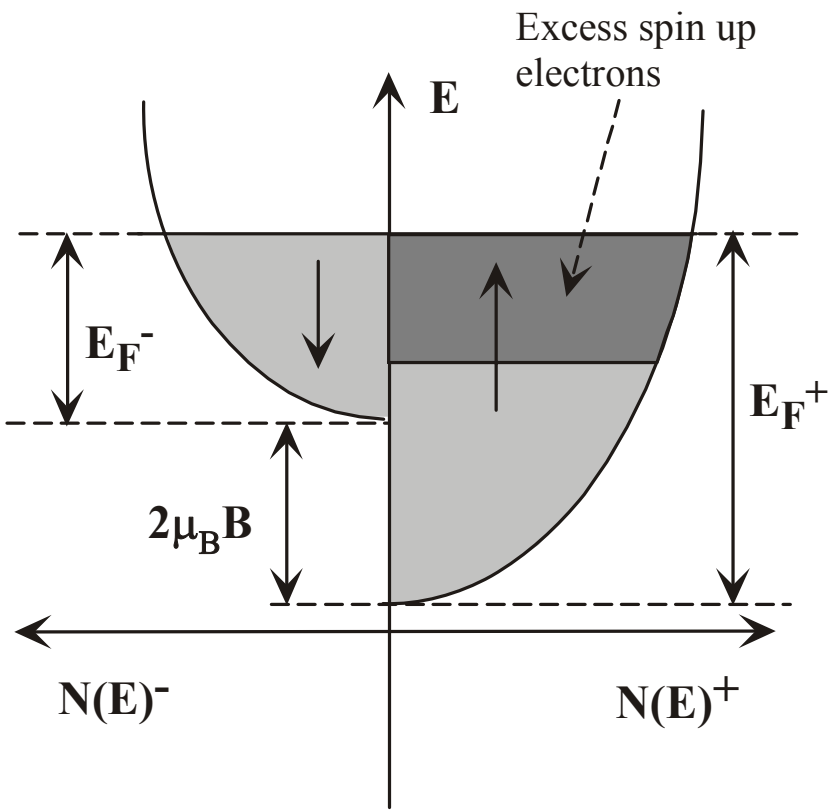

(b)

\section{Figure 26}

Density of states vs. energy for the two spin components. In the non magnetic states (a), the occupancy by spin up and spin down electrons is the same. In Pauli magnetism at absolute zero when a magnetic field is applied, the spin down and spin up electrons are moved away from each other which leads to unparity in that the number of electrons with one type of spin would dominate over the other (b). The effect of the applied magnetic field is amplified to show the point. In ferromagnetic material and below the Curie temperature the effective field or the molecular field is so large, thus this splitting is sizeable and no external magnetic field is needed for the shift shown. 
Özgür et al., submitted to Journal of Materials Science: Materials in Electronics, 2009 Microwave Ferrites, Part 1: Fundamental properties

Ü. Özgür et al.

Journal of Materials Science: Materials in Electronics

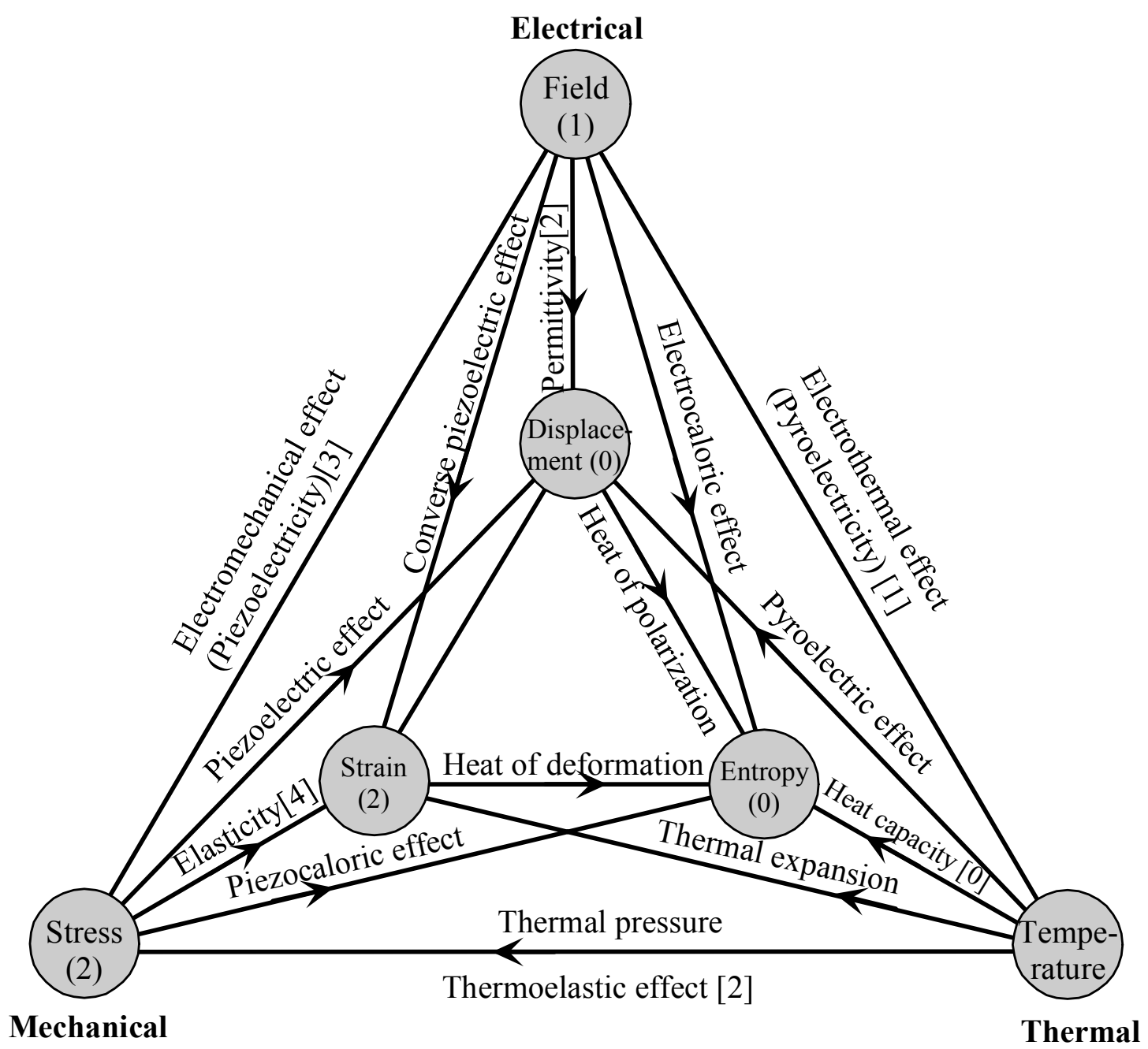

\section{Figure 27}

The well-known triangle used to describe the pathways between mechanical, electrical, and thermal energies in a class of non-centro-symmetric materials exhibiting piezoelectric effect, pyroelectric effect (converse of electrocaloric effect), piezocaloric effect (converse of thermal expansion), pyromagnetic effect (converse of magnetocaloric effect), and magnetoelectric polarizability so on. For simplicity, the magnetic property node is not shown. Tensor rank of the variable is shown in parentheses and the tensor rank of the property is shown in brackets. (Motivated by Nye.) 
Ü. Özgür et al.

Journal of Materials Science: Materials in Electronics

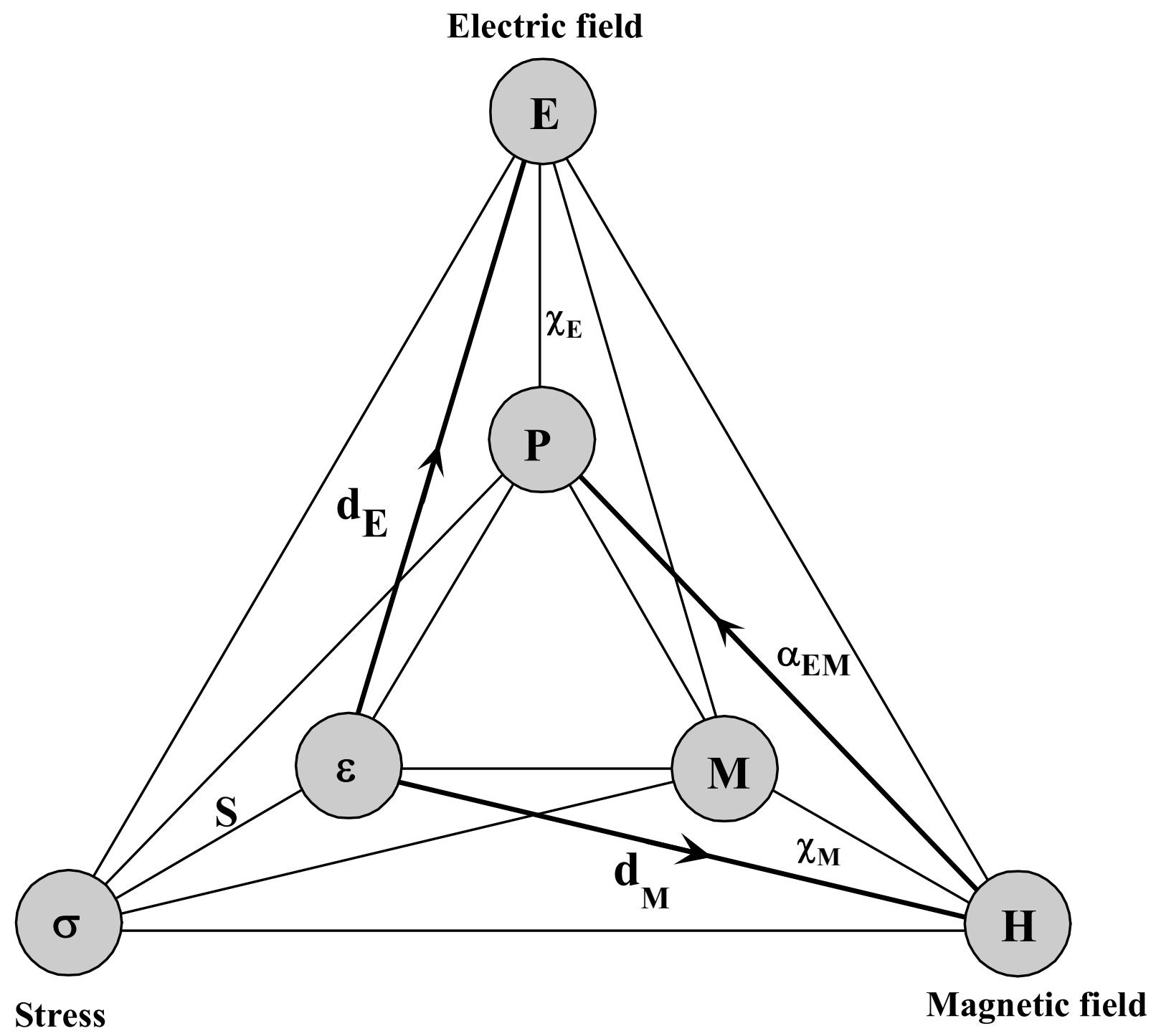

\section{Figure 28}

The well-known triangle used to describe the pathways between properties such as electric field, polarization, and magnetic field with connected properties such as strain $(\varepsilon)$, electrical polarization $(\mathrm{P})$, and magnetization $(\mathrm{M})$. Coupling coefficients between the various properties and external forces are also shown: electric susceptibility $\left(\chi_{E}\right)$, magnetic susceptibility $\left(\chi_{M}\right)$, piezoelectricity $\left(\mathrm{d}_{\mathrm{E}}\right)$ and magnetostriction $\left(\mathrm{d}_{\mathrm{M}}\right)$ tensors, and compliance tensor (S). $\alpha_{\mathrm{EM}}$ depicts the coupling between magnetic field and the induced electric polarization thus the electric field. For simplicity, the optical property node and its effect on the other properties or vice versa are not shown. 
Özgür et al., submitted to Journal of Materials Science: Materials in Electronics, 2009 Microwave Ferrites, Part 1: Fundamental properties 
Özgür et al., submitted to Journal of Materials Science: Materials in Electronics, 2009 Microwave Ferrites, Part 1: Fundamental properties

Ü. Özgür et al.

Journal of Materials Science: Materials in Electronics

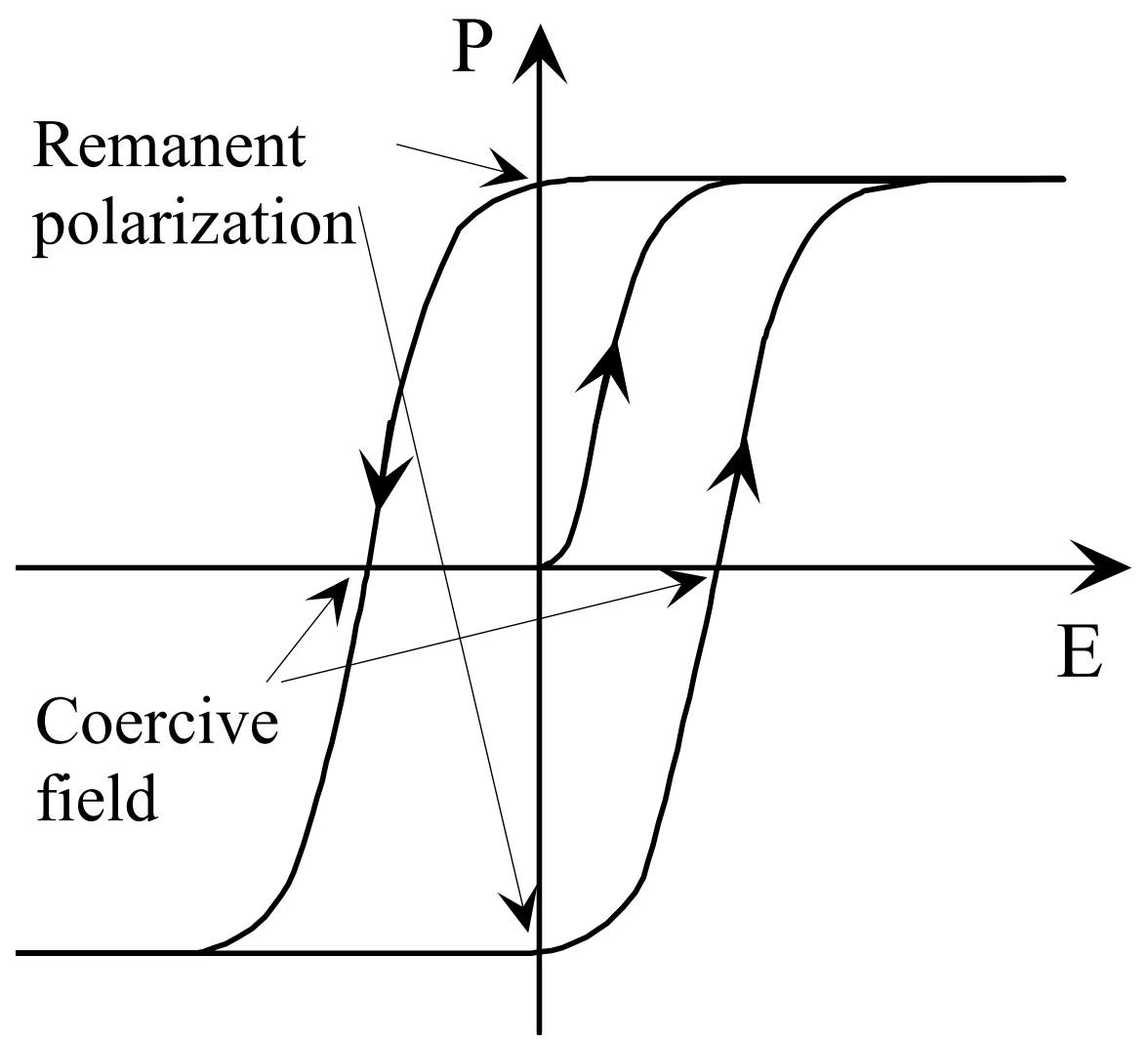

Figure 29

Hysteresis loop in the electric field vs. polarization curve in a ferroelectric material. Upon application of an electric field in the positive direction, the polarization increases and when all the domains are oriented, the polarization saturates. When the field strength is reduced the direction of the polarization remains until a specific reverse field, coercive field, which forces the domains to charge direction, is reached. As the field strength is increased further, the polarization again saturates. As the field intensity is reduced the net polarization remains in the same direction until a specific positive field, coercive field, which forces all the domains to switch polarization direction. The polarization remaining at zero field is called the remanent polarization. 
Ü. Özgür et al.

Journal of Materials Science: Materials in Electronics

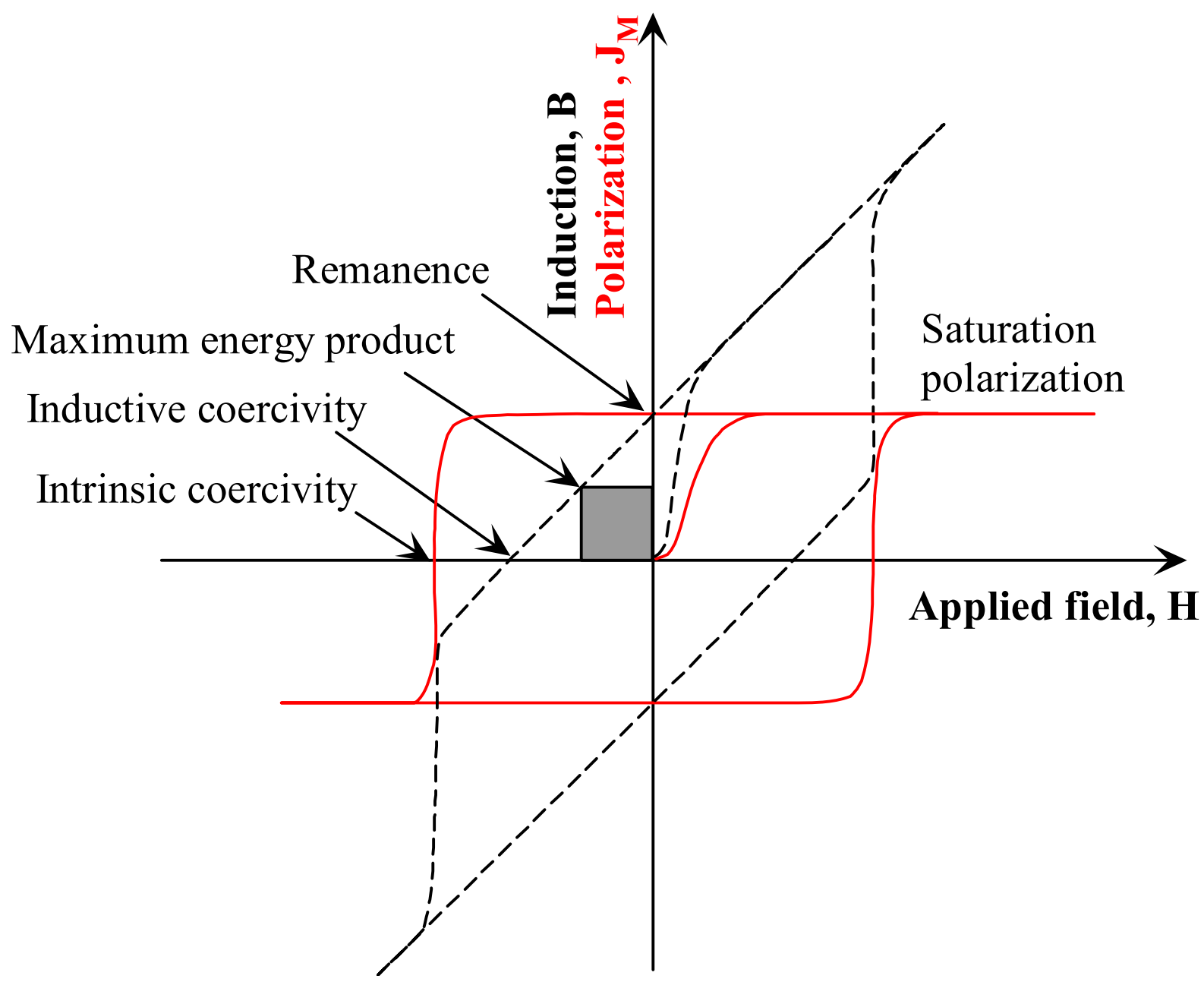

\section{Figure 30}

Hysteresis loop in the magnetization vs. magnetic field curve in a ferromagnetic/ferrimagnetic material. Upon application of a magnetic field, the magnetization increases and when all the domains (magnetic dipoles) are oriented coherently, the magnetization saturates. When the magnetic field is reduced the magnetization does not reduce immediately. The direction is switched only after a specific reverse magnetic field, coercive field, is reached. As the field is increased further in the negative direction, the magnetization again saturates. As the magnetic field intensity is reduced the net polarization remains in the same direction until a specific positive field, coercive field, which forces all the domains to switch polarization direction, is reached. The magnetization remaining at zero field is called the remanent 
Özgür et al., submitted to Journal of Materials Science: Materials in Electronics, 2009 Microwave Ferrites, Part 1: Fundamental properties

magnetization. (The red solid hysteresis curve is for intrinsic magnetic polarization (intensity of magnetization) $J_{M}=\mu_{0} M=B-\mu_{0} H$. The blue black dashed curve one is the sum of intrinsic and permeability curves together:. $B=\mu_{0} H+J_{M}=\mu_{0} H+\mu_{0} M$. The maximum $\mathrm{BH}$ product $\left(\mathrm{kJ} / \mathrm{m}^{3}\right)$ indicated by the gray area represents the maximum energy storage per unit volume and is the figure of merit most often used for permanent magnet materials). 
Özgür et al., submitted to Journal of Materials Science: Materials in Electronics, 2009 Microwave Ferrites, Part 1: Fundamental properties

Ü. Özgür et al.

Journal of Materials Science: Materials in Electronics

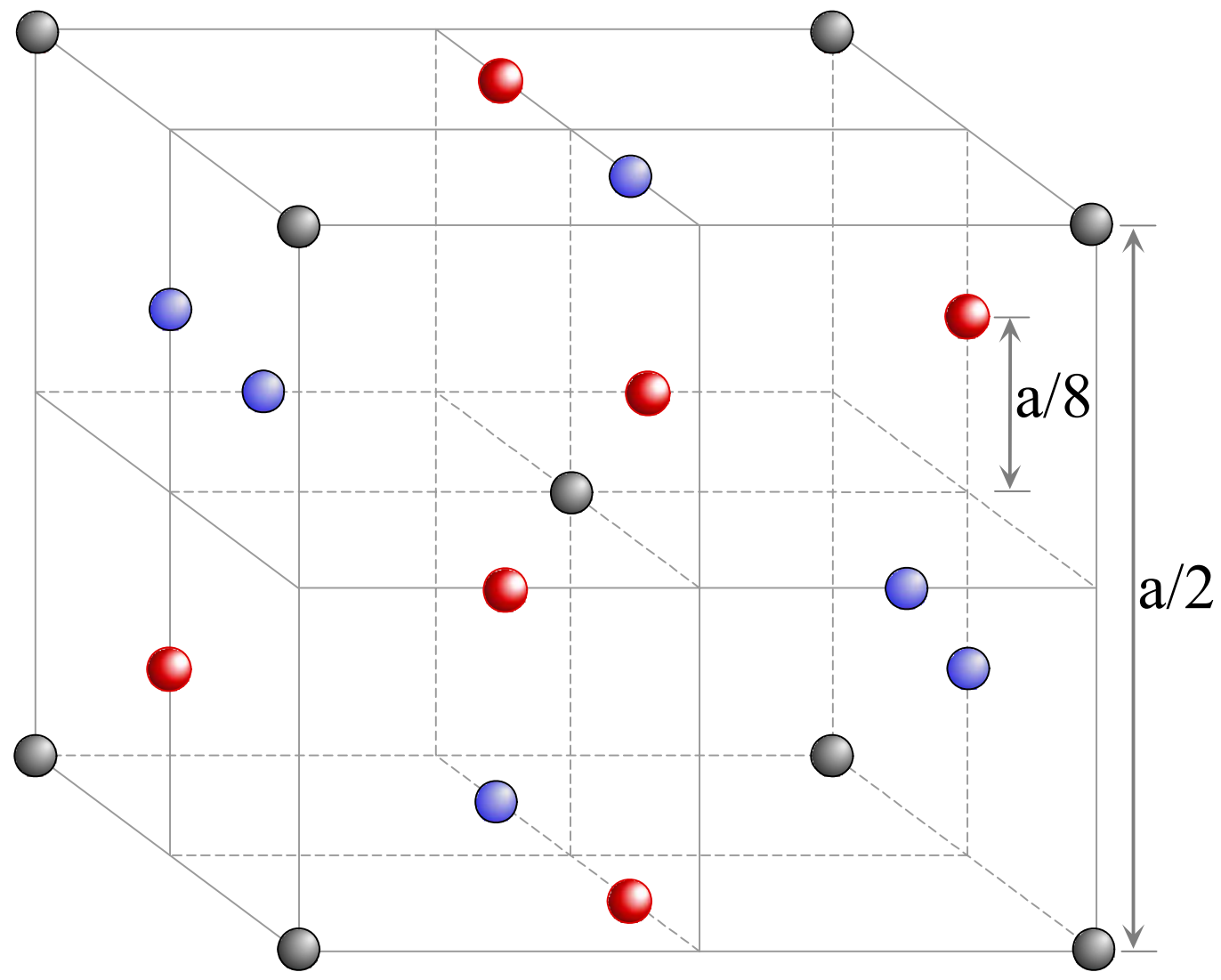

\section{$\mathrm{Fe}^{3+}(a)$ \\ Octahedral site}

$\mathrm{Fe}^{2+}(d)$
Tetrahedral site

\section{$\mathrm{RE}^{3+}(c)$
Dodecahedral site}

\section{Figure 31}

Schematic of an octant of a garnet crystal structure (lattice constant "a") showing only cation positions. 
Özgür et al., submitted to Journal of Materials Science: Materials in Electronics, 2009 Microwave Ferrites, Part 1: Fundamental properties

Ü. Özgür et al.

Journal of Materials Science: Materials in Electronics

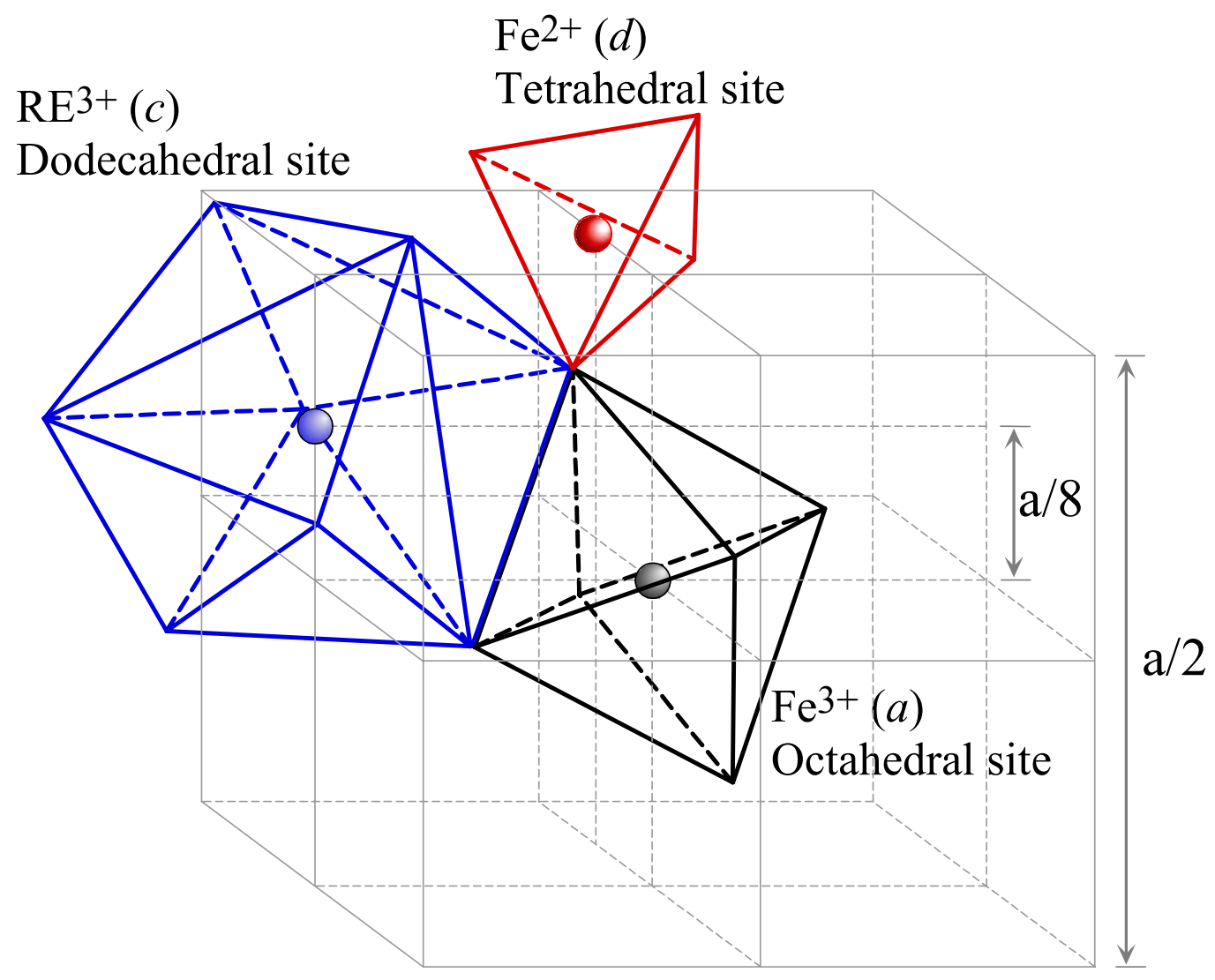

Figure 32

An octant of a garnet crystal structure (lattice constant "a") showing a trivalent ion of iron on a site surrounded by six oxygen ions in octahedral symmetry, a divalent ion of iron on a site surrounded by four oxygen ions in tetrahedral symmetry, and a rare-earth ion surrounded by 8 oxygen ions which form an 8-cornered 12-sided polyhedron. (After Geller and Gilleo.) 
Özgür et al., submitted to Journal of Materials Science: Materials in Electronics, 2009 Microwave Ferrites, Part 1: Fundamental properties

Ü. Özgür et al.

Journal of Materials Science: Materials in Electronics

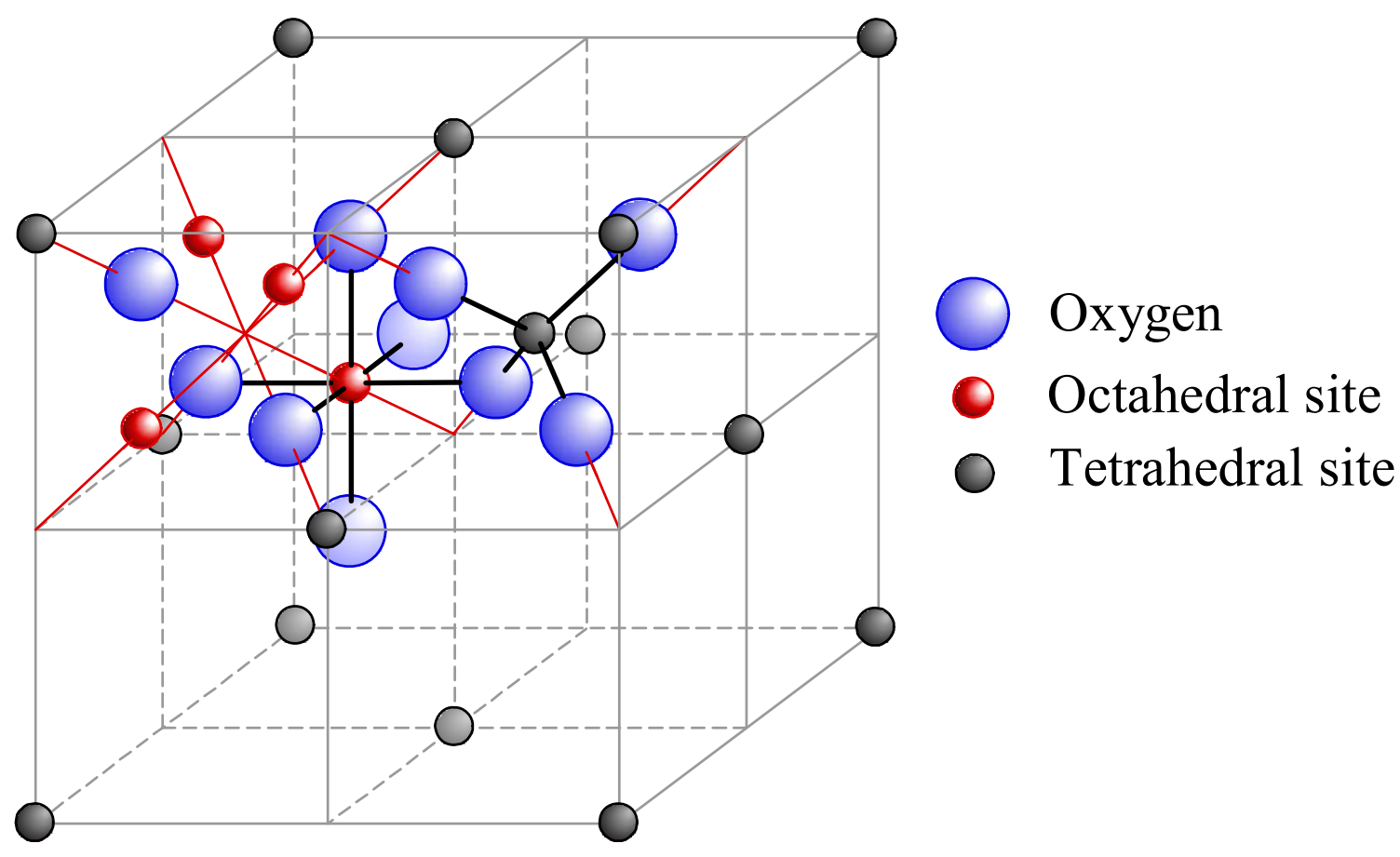

(a)

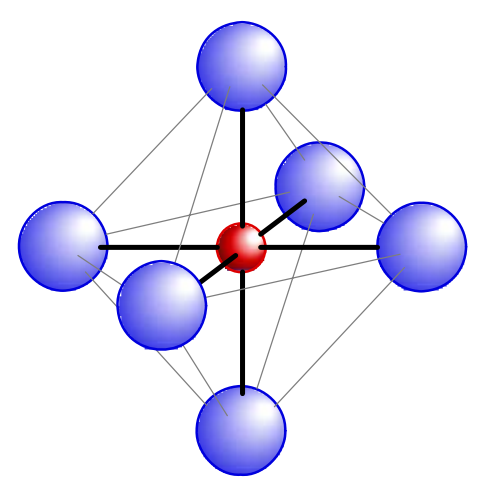

(b)

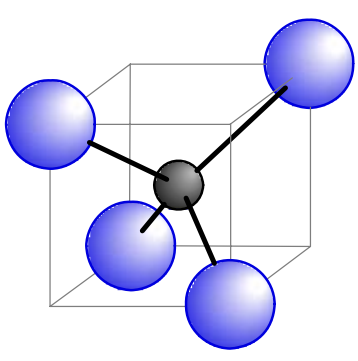

(c)

Figure 33

(a) Spinel unit cell structure, (b) octahedral interstice (B site: 32 per unit cell, 16 occupied), and (c) tetrahedral interstice (A site: 64 per unit cell, 8 occupied). In (a) the ionic positions are the same in octants sharing only one edge and different in octants 
Özgür et al., submitted to Journal of Materials Science: Materials in Electronics, 2009 Microwave Ferrites, Part 1: Fundamental properties

sharing a face. Each octant contains 4 Oxygen ions. In (a) ionic positions in only two adjacent octants are shown, where the octant on the left contains octahedral and the one on the right contains tetrahedral sites. All ions are positioned on body diagonals of the octants and the octant on the right contains a tetrahedral site at the octant center. 
Özgür et al., submitted to Journal of Materials Science: Materials in Electronics, 2009 Microwave Ferrites, Part 1: Fundamental properties

Ü. Özgür et al.

Journal of Materials Science: Materials in Electronics

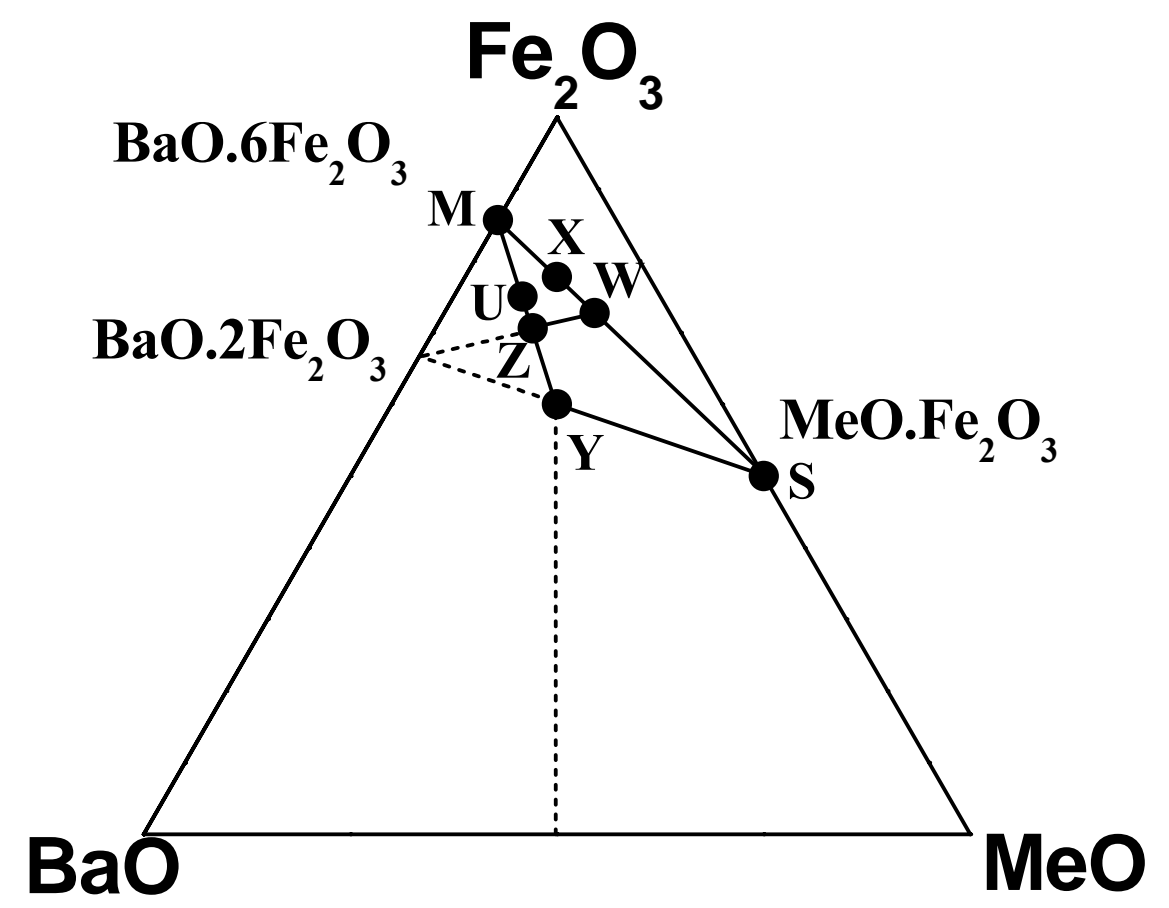

Figure 34

Chemical composition diagram showing how the hexagonal ferrites are derived from the spinel $\mathrm{MeO} \cdot \mathrm{Fe}_{2} \mathrm{O}_{3}$ structure. 
Özgür et al., submitted to Journal of Materials Science: Materials in Electronics, 2009 Microwave Ferrites, Part 1: Fundamental properties

Ü. Özgür et al.

Journal of Materials Science: Materials in Electronics

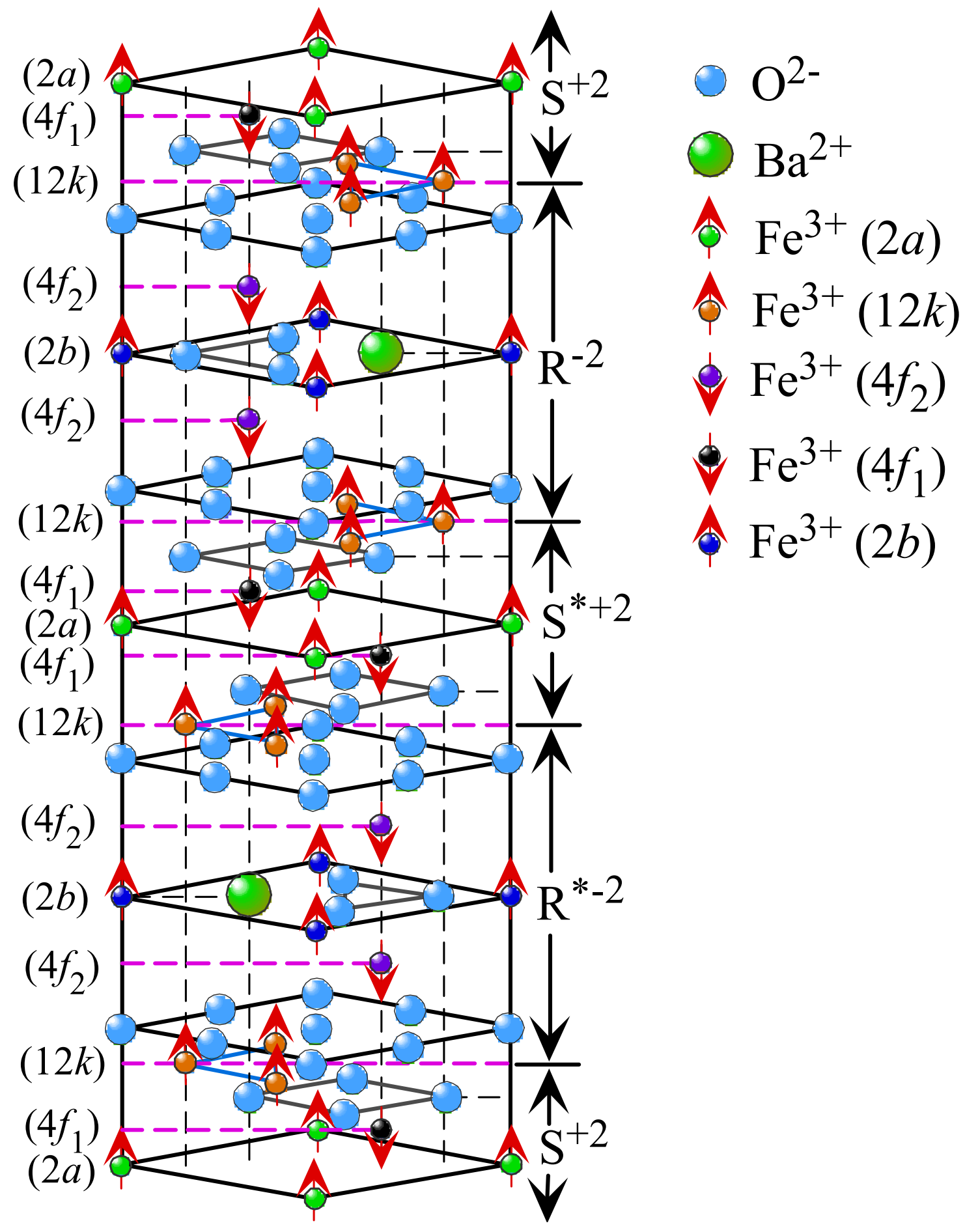


Özgür et al., submitted to Journal of Materials Science: Materials in Electronics, 2009 Microwave Ferrites, Part 1: Fundamental properties

\section{Figure 35}

The schematic structure of the hexaferrite $\mathrm{BaFe}_{12} \mathrm{O}_{19}$. The arrows on $\mathrm{Fe}$ ions represent the direction of spin polarization. $2 a, 12 k$, and $4 f_{2}$ are octahedral, $4 f_{1}$ are tetrahedral, and $2 b$ are hexahedral (trigonal bipyramidal) sites. The unit cell contains a total of $38 \mathrm{O}^{2-}$ ions, 2 $\mathrm{Ba}^{2+}$ ions, and $24 \mathrm{Fe}^{3+}$ ions. $\mathrm{Fe}^{3+}$ ions in $12 k, 2 a$, and $2 b$ sites (16 total per unit cell) have their spins up, while the $\mathrm{Fe}^{3+}$ ions in $4 f_{1}$ and $4 f_{2}$ sites ( 8 total per unit cell) have their spins down, which results in a net total of 8 spins up, and therefore, a total moment of $8 \times 5 \mu_{\mathrm{B}}$ $=40 \mu_{\mathrm{B}}$ per unit cell that contains two $\mathrm{Ba}^{2+}$ ions. The $\mathrm{R}$ and $\mathrm{S}$ sub-units shown have chemical formulae $\mathrm{R}=\left(\mathrm{Ba}^{2+} \mathrm{Fe}_{6}^{3+} \mathrm{O}_{11}^{2-}\right)^{2-}$ and $\mathrm{S}=\left(\mathrm{Fe}_{6}^{3+} \mathrm{O}_{8}^{2-}\right)^{2+}$. The asterix $\left(^{*}\right)$ indicates that the corresponding sub-unit is rotated $180^{\circ}$ around the hexagonal axis. 
Özgür et al., submitted to Journal of Materials Science: Materials in Electronics, 2009 Microwave Ferrites, Part 1: Fundamental properties

Ü. Özgür et al.

Journal of Materials Science: Materials in Electronics

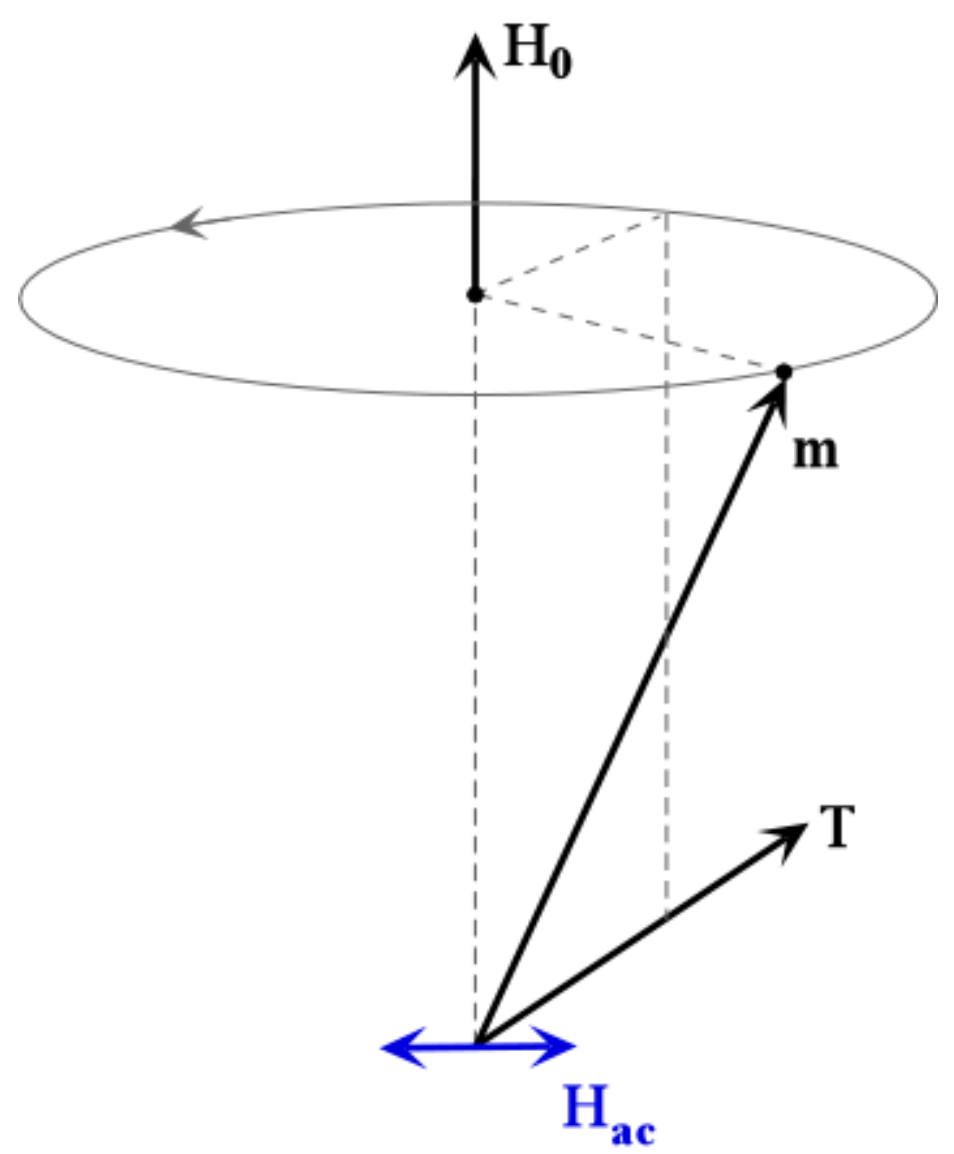

\section{Figure 36}

A schematic of dipole moment $\mathbf{m}$ precessing about a static magnetic field $\mathbf{H}_{\mathbf{0}}$. When the frequency of the alternating magnetic field $\mathbf{H}_{\mathbf{a c}}$ (applied normal to the static field) is near the natural precession frequency the precession of the magnetic moment grows. 
Özgür et al., submitted to Journal of Materials Science: Materials in Electronics, 2009 Microwave Ferrites, Part 1: Fundamental properties

Ü. Özgür et al.

Journal of Materials Science: Materials in Electronics
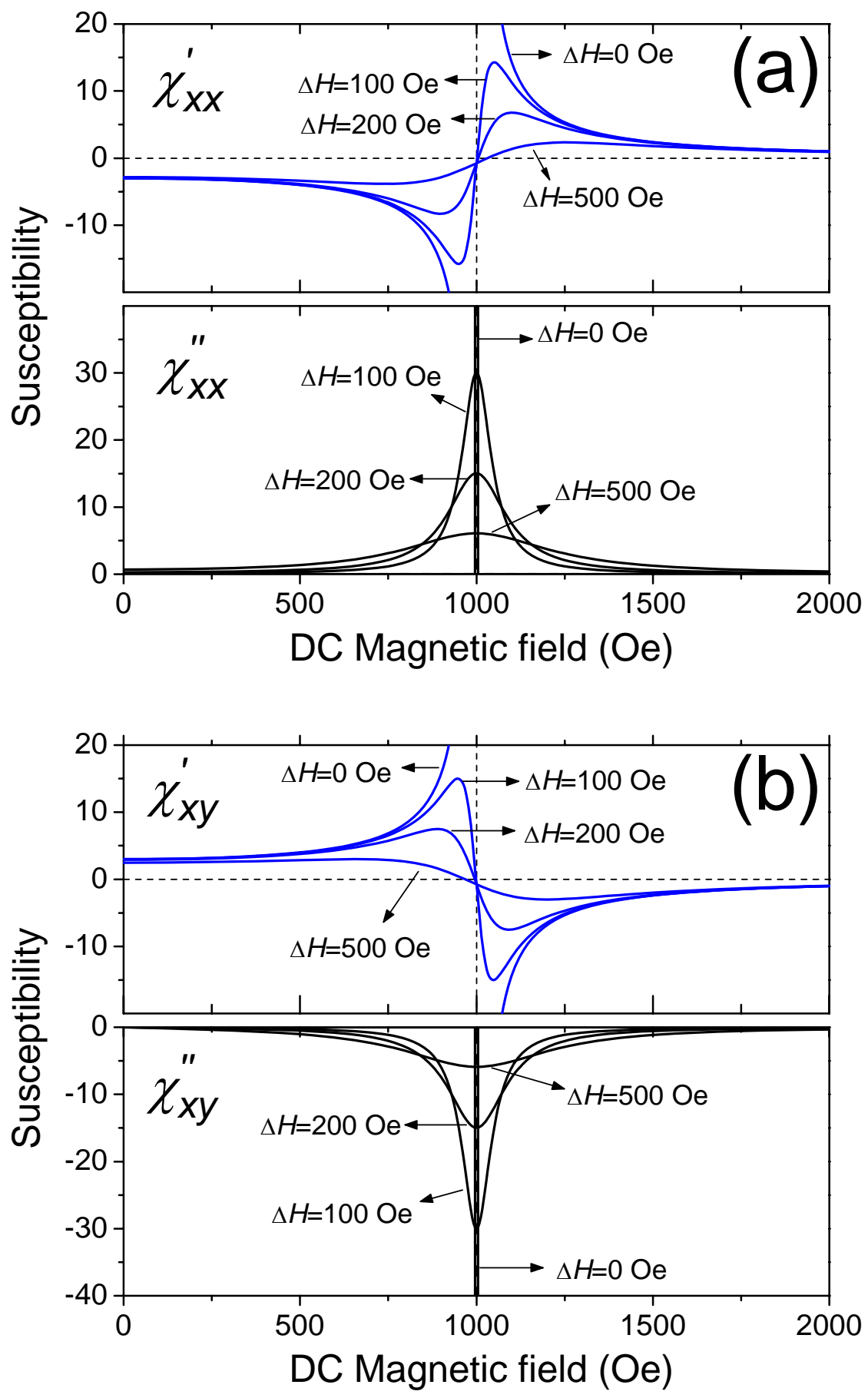
Özgür et al., submitted to Journal of Materials Science: Materials in Electronics, 2009 Microwave Ferrites, Part 1: Fundamental properties

\section{Figure 37}

The real (dispersive) and the imaginary (dissipative) components of the susceptibility tensor elements computed for different damping factors $\left(\phi=\gamma \mu_{0} \Delta H / 2 \omega_{0}=2 \phi H_{0}\right)$ using a resonance frequency of $2.8 \mathrm{GHz}$ and a saturation magnetization of $4 \pi \mathrm{M}_{\mathrm{s}}=3000$ Gauss. The medium has been assumed to be spherical and magnetically saturated at low fields. 
Özgür et al., submitted to Journal of Materials Science: Materials in Electronics, 2009 Microwave Ferrites, Part 1: Fundamental properties

Ü. Özgür et al.

Journal of Materials Science: Materials in Electronics

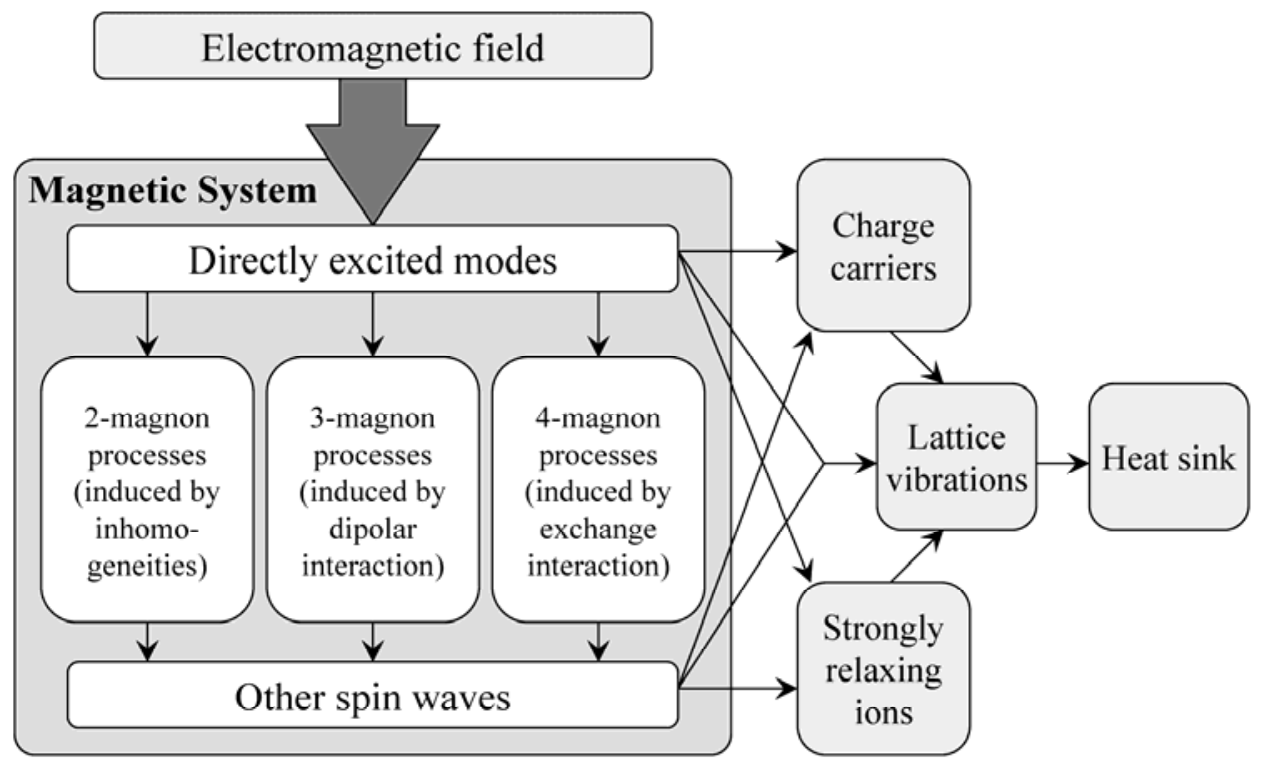

\section{Figure 38}

A pictorial overview of the magnetic relations processes that affect the FMR damping in ferrites. (After Schloemann.) 
Özgür et al., submitted to Journal of Materials Science: Materials in Electronics, 2009 Microwave Ferrites, Part 1: Fundamental properties

Ü. Özgür et al.

Journal of Materials Science: Materials in Electronics

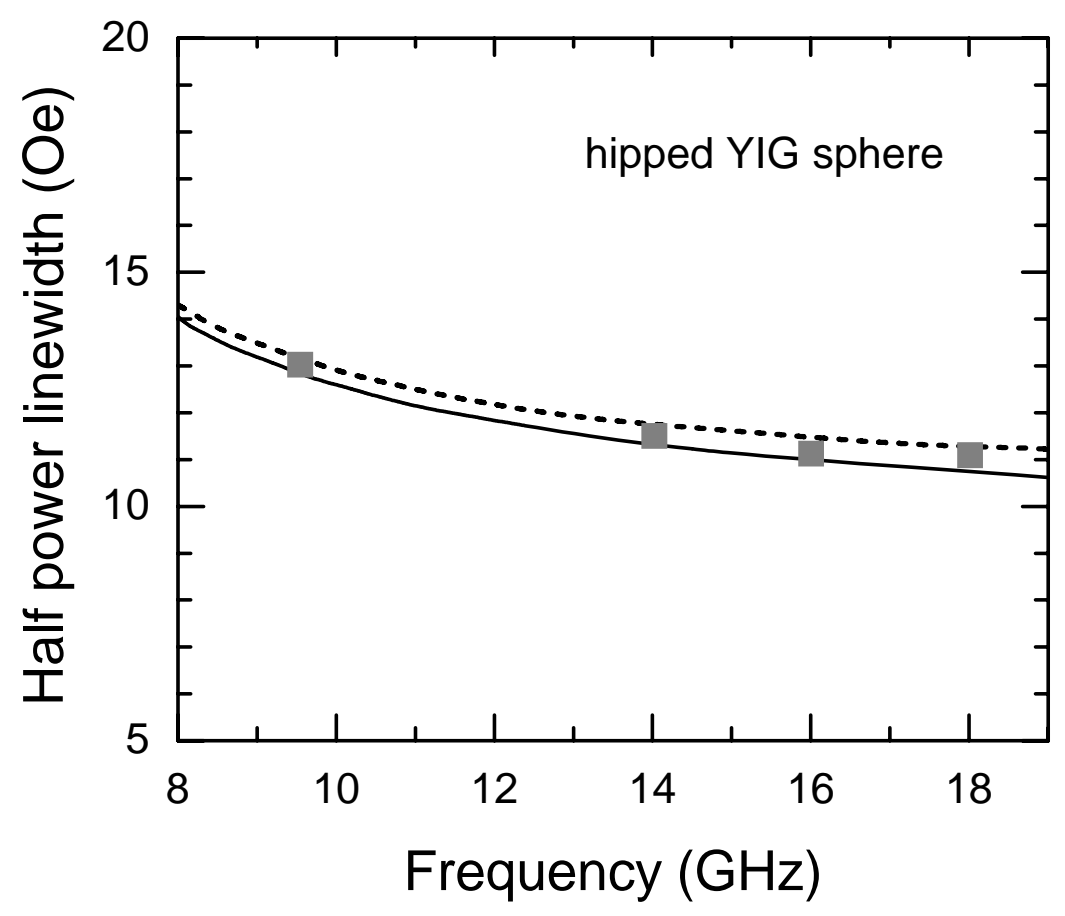

Figure 39

FMR half power linewidth of hipped YIG sphere of $2.04 \mathrm{~mm}$ diameter. The solid squares show the data, the solid line is the calculated linewidths for the two-magnon anisotropy scattering process, and the dashed line shows the two-magnon response shifted upward by $0.03 f$ to account for the intrinsic linewidth of 0.3 Oe. (After Nazarov et al.) 
Ü. Özgür et al.

Journal of Materials Science: Materials in Electronics

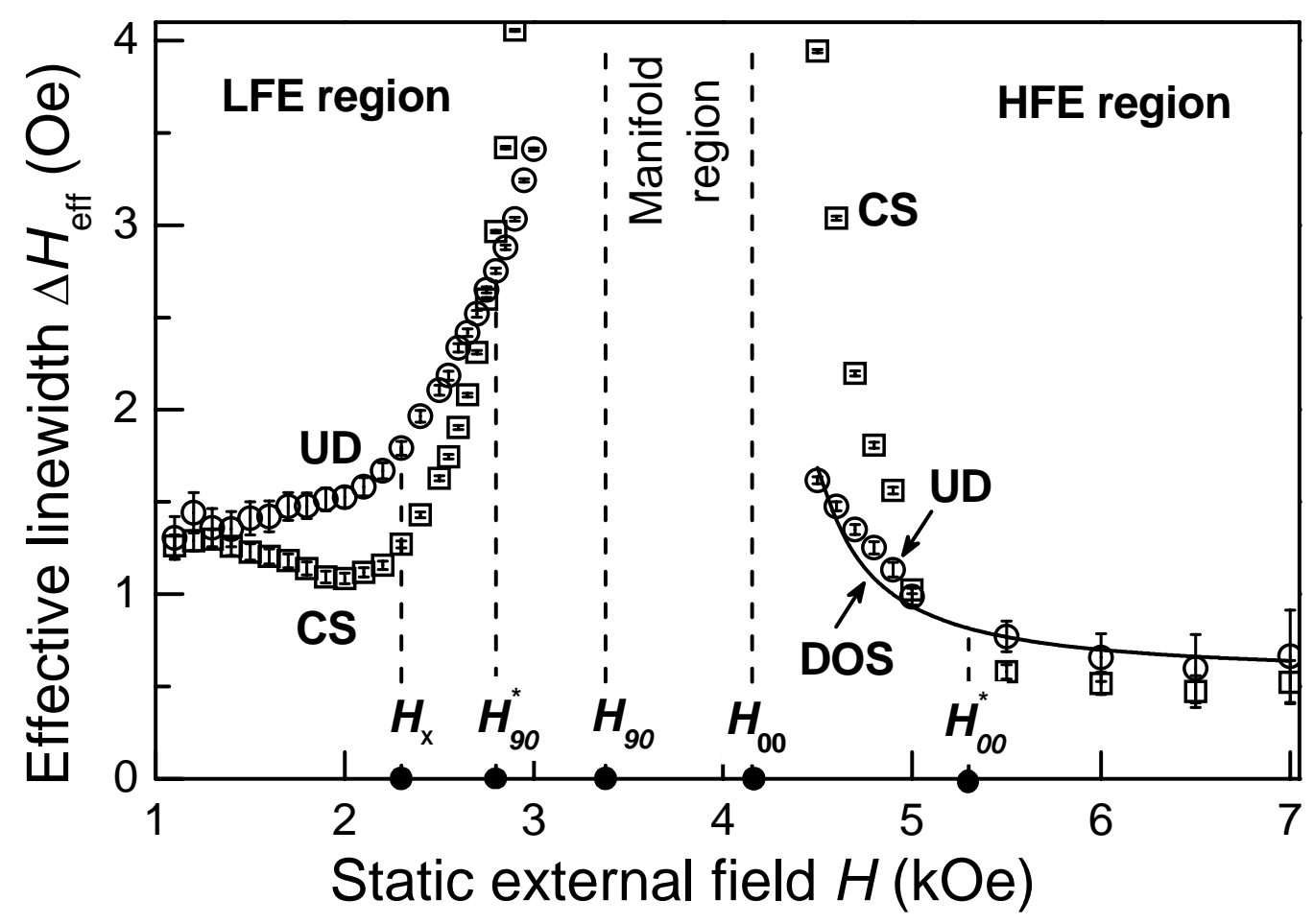

Figure 40

Effective linewidth for conventionally sintered (CS) and ultra dense (UD) YIG sphere samples measured outside the manifold region at a signal drive frequency of $10 \mathrm{GHz}$. (After Mo et al.) 
Özgür et al., submitted to Journal of Materials Science: Materials in Electronics, 2009 Microwave Ferrites, Part 1: Fundamental properties

Ü. Özgür et al.

Journal of Materials Science: Materials in Electronics
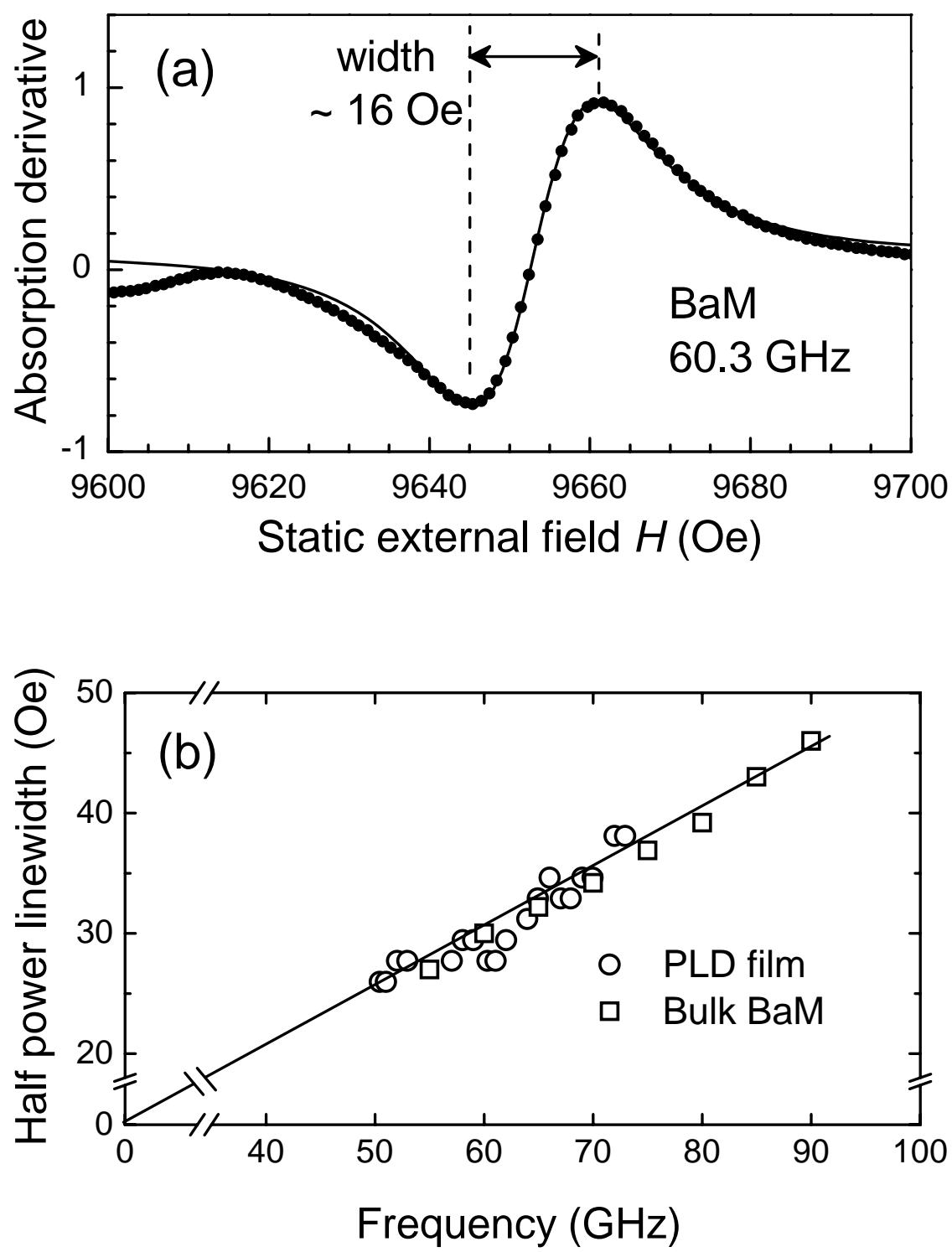

\section{Figure 41}

(a) Absorption derivative versus the applied static magnetic field at $60.3 \mathrm{GHz}$ for the main FMR mode in a PLD grown $0.85 \mu$ m-thick BaM film. The closed circles show the data and the solid line is a fit based on a Lorentzian absorption response. (b) Half power linewidth for the same FMR mode as a function of the frequency. The data for bulk BaM 
Özgür et al., submitted to Journal of Materials Science: Materials in Electronics, 2009 Microwave Ferrites, Part 1: Fundamental properties

from Karim et al. are shown with squares. The solid line is a linear fit to all the data. (After Song et al.) 
Özgür et al., submitted to Journal of Materials Science: Materials in Electronics, 2009 Microwave Ferrites, Part 1: Fundamental properties

Ü. Özgür et al.

Journal of Materials Science: Materials in Electronics

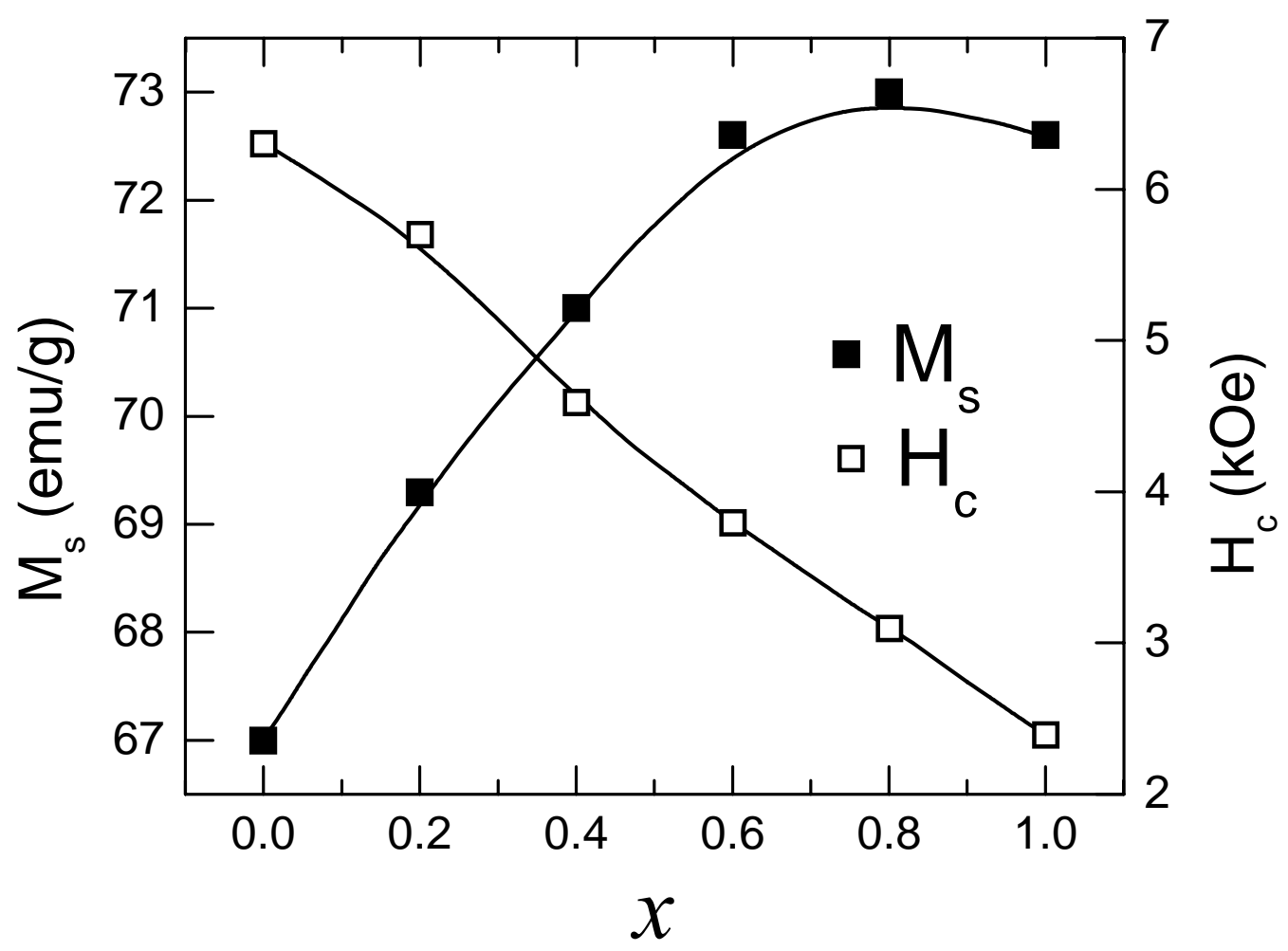

Figure 42

Saturation magnetization, $M_{s}$, and coercivity, $H_{c}$, of $\operatorname{Sr}\left[\mathrm{Zn}_{0.7} \mathrm{Nb}_{0.3}\right]_{x} \mathrm{Fe}_{12-x} \mathrm{O}_{19}$ as a function of $x$, for samples annealed at $950{ }^{\circ} \mathrm{C} / 5 \mathrm{~h}$. (After Fang et al.) 
Özgür et al., submitted to Journal of Materials Science: Materials in Electronics, 2009 Microwave Ferrites, Part 1: Fundamental properties

Ü. Özgür et al.

Journal of Materials Science: Materials in Electronics

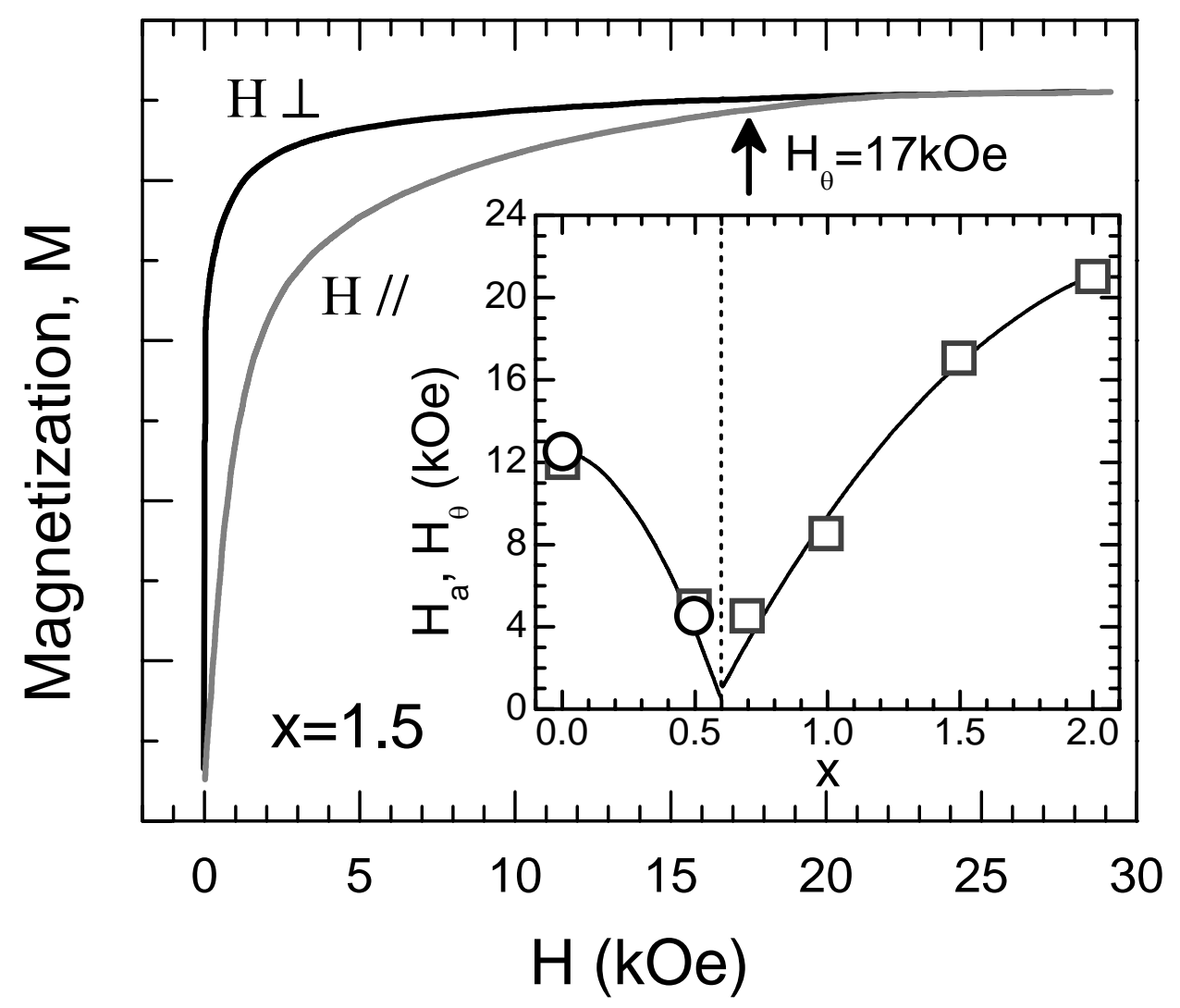

\section{Figure 43}

The magnetization curves parallel and perpendicular to the magnetic alignment direction used during sample preparation (surface normal) for a $\mathrm{BaZn}_{2-x} \mathrm{Co}_{x} \mathrm{Fe}_{16} \mathrm{O}_{27}$ sample with $x$ $=1.5$. The field corresponding to the intersection of the two curves is the anisotropy field $H_{a}$ for samples with $c$-axis anisotropy or $H_{\theta}$ for samples with $c$-plane anisotropy. Inset shows the anisotropy field $H_{a}$ (c-axis) or $H_{\theta}$ (c-plane) for various Co compositions, $x$. The values of anisotropy fields were determined based on two methods: the magnetization curves parallel and perpendicular to the magnetic alignment direction (open squares) and the law of approach to saturation magnetization (open circles). (After Li et al.) 
Özgür et al., submitted to Journal of Materials Science: Materials in Electronics, 2009 Microwave Ferrites, Part 1: Fundamental properties

Ü. Özgür et al.

Journal of Materials Science: Materials in Electronics

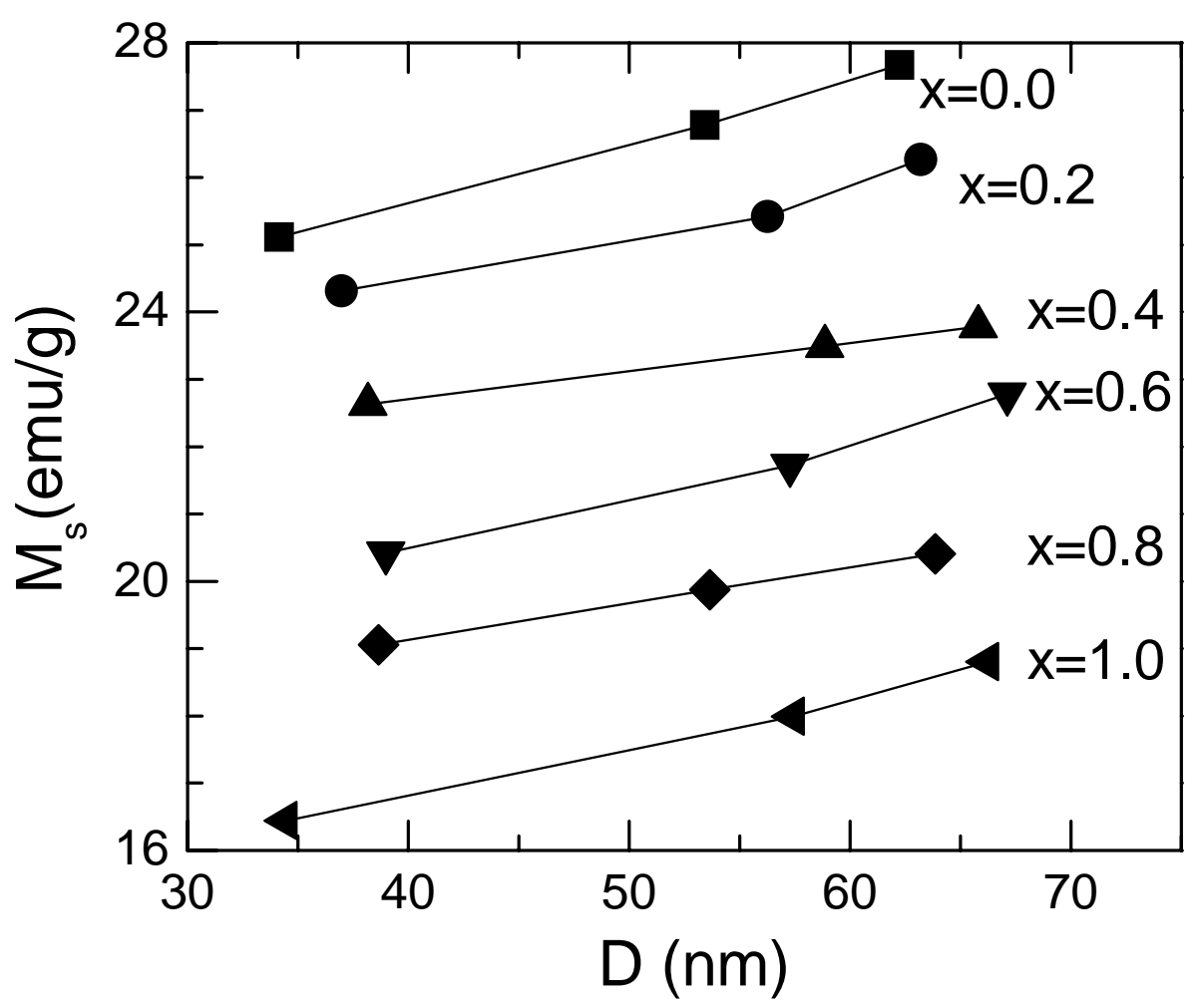

\section{Figure 44}

The variation of saturation magnetization $\left(\mathrm{M}_{\mathrm{s}}\right)$ with the average particle size (D) in $\mathrm{Y}_{3-x} \mathrm{Gd}_{x} \mathrm{Fe}_{5} \mathrm{O}_{12}$ prepared by sol-gel. (After Cheng et al.) 
Özgür et al., submitted to Journal of Materials Science: Materials in Electronics, 2009 Microwave Ferrites, Part 1: Fundamental properties

Ü. Özgür et al.

Journal of Materials Science: Materials in Electronics

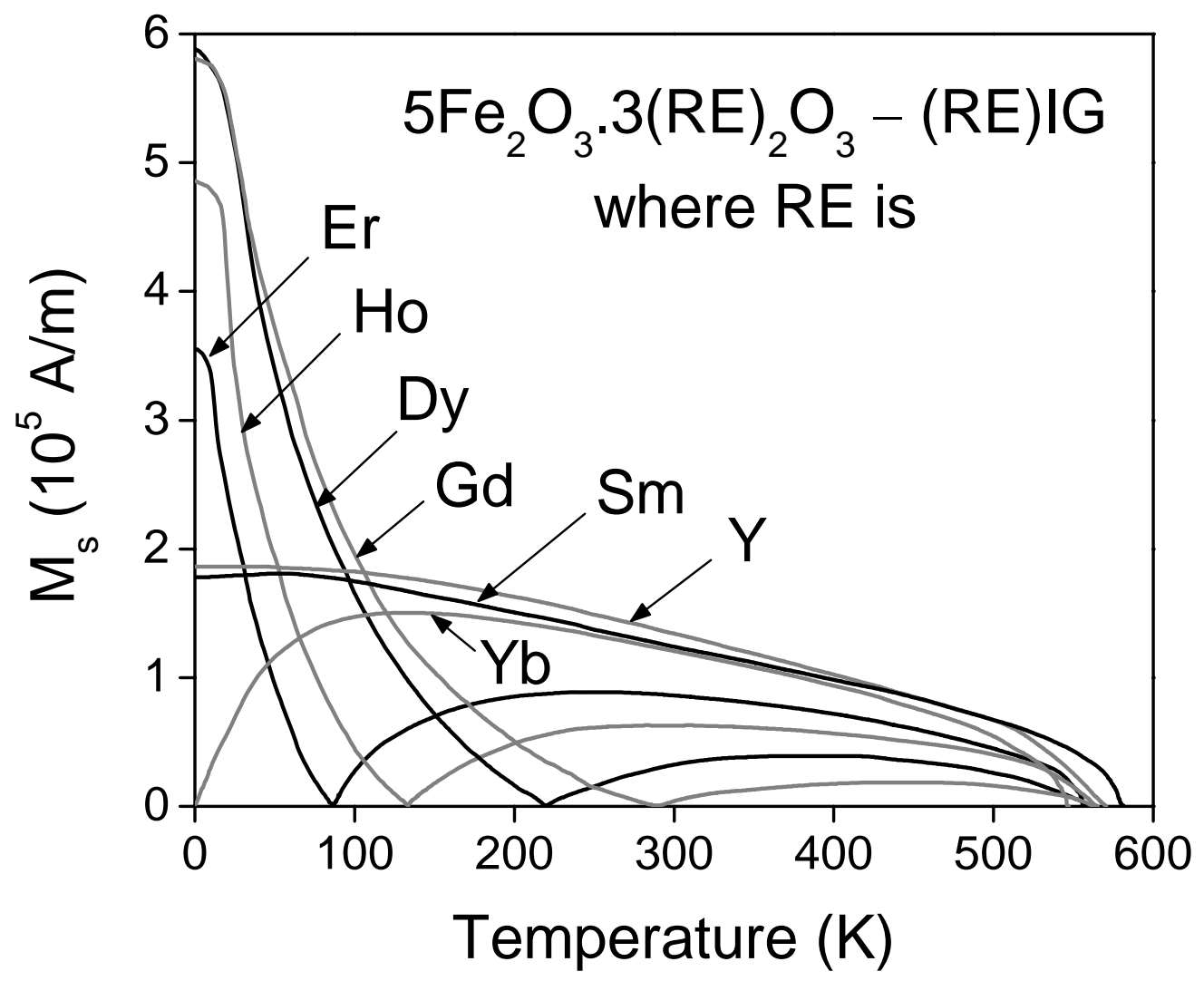

Figure 45

Spontaneous magnetization for the family of ferromagnetic garnets as a function of temperature. (After Rodrigue.) 
Ü. Özgür et al.

Journal of Materials Science: Materials in Electronics
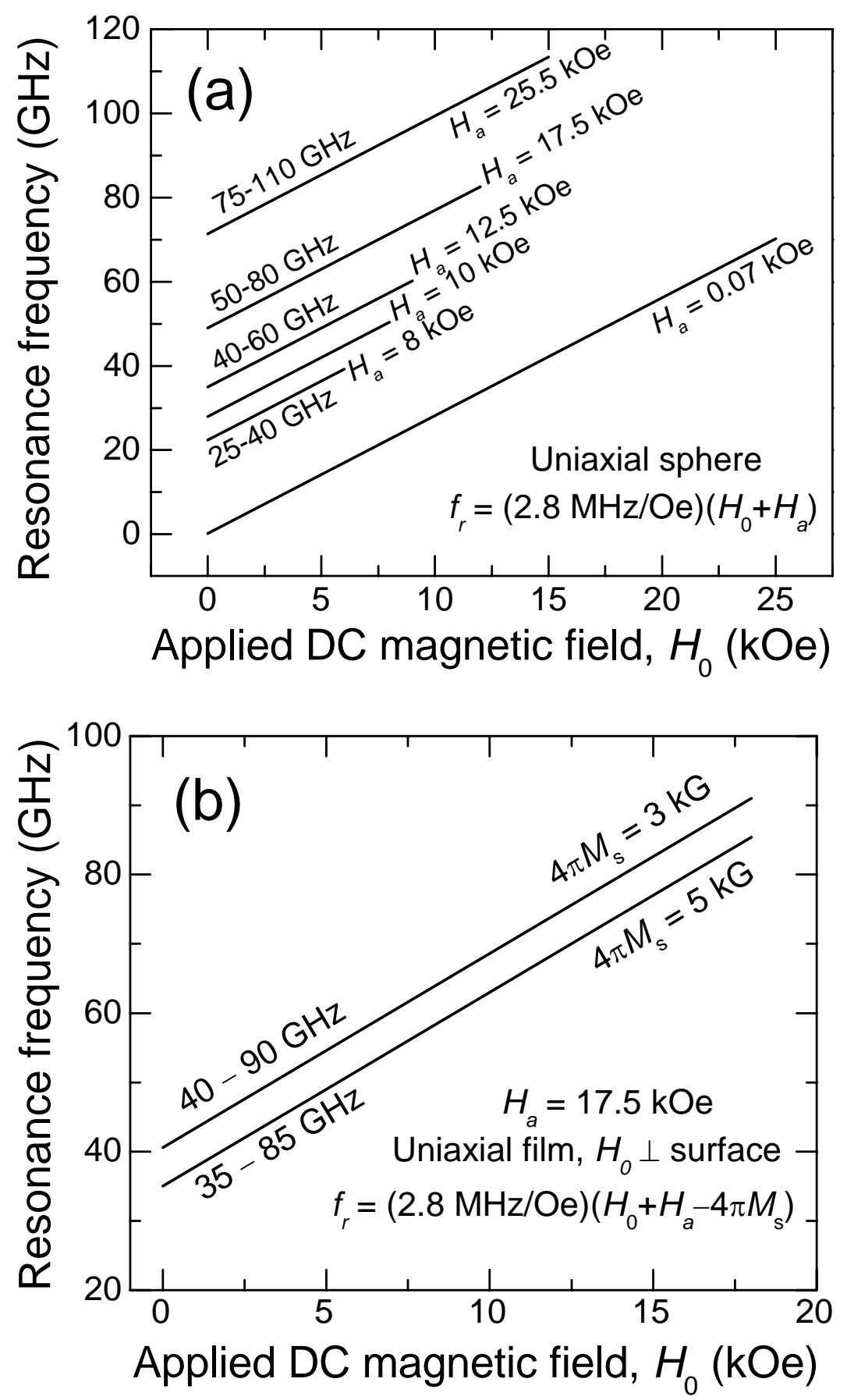

Figure 46

Ferrite resonance frequency as a function of the applied field $H_{0}$ (a) for different values of anisotropy, $H_{a}$, in a spherical specimen and (b) for different values of saturation magnetization, $4 \pi M_{s}$, in a thin film. 
Özgür et al., submitted to Journal of Materials Science: Materials in Electronics, 2009 Microwave Ferrites, Part 1: Fundamental properties

\section{References}

${ }^{1}$ R. E. Collin, "Foundations for Microwave Engineering", $2^{\text {nd }}$ Ed., IEEE Press, 2000.

${ }^{2}$ K. H. J. Buschow, Rep. Prog. Phys. 54, 1123 (1991).

${ }^{3}$ Ü. Özgür, Y. Alivov, and H. Morkoç, "Microwave Ferrites, Part 2: Passive components and electrical tuning", to be published in J. Mater. Sci.: Materials in Electronics, 2009.

${ }^{4}$ See for example, C. Kittel, Introduction to Solid State Physics, $7^{\text {th }}$ edition, Wiley, 1996; J. S. Blakemore, "Solid State Physics", $2^{\text {nd }}$ Edition, Cambridge University Press, 1985; Walter Beam, "Electronics of Solids", McGraw Hill, 1965; S. O. Kasap, "Principles of Electronic Material and Devices" $3^{\text {rd }}$ Edition, McGrawHill, 2002.

${ }^{5}$ N. W. Ashcroft and N. D. Mermin, "Solid State Physics", Holt-Reinhart-Winston, 1976.

${ }^{6}$ C. Kittel, Rev. Mod. Physics 21, 541 (1949).

${ }^{7}$ J. F. Nye, "Physical Properties of Crystals: Their Representation by Tensors and Matrices", Oxford University Press, Oxford, 1998.

${ }^{8}$ T. Mishima, U. S. Patent 2,2027,966; Ohm, 19, 353 (1932)

${ }^{9}$ A. H. Geisler, Rev. Mod. Phys. 25, 316 (1953).

${ }^{10}$ S. Geller and M. A. Gilleo, J. Phys. Chem. Solids 3, 30 (1957).

${ }^{11}$ M. A. Gilleo and S. Geller, Phys. Rev. 110, 73 (1958).

${ }^{12}$ J. J. Went, G. W. Ratheneau, E. W. Gorter, and G. W. Van Oosterhout, Philips Tech. Rev. 13, 194 (1952).

${ }^{13}$ H. P. J. Wijn, Nature 170, 707 (1952).

${ }^{14}$ P. B. Braun, Nature 170, 708 (1952).

${ }^{15}$ G. H. Jonker, H. P. Wijn and P. B. Braun, Philips. Tech. Rev. 18, 145 (1956-1957)

${ }^{16}$ D. Lisjak and M. Drofenik, J. Am. Ceram. Soc. 90, 3517 (2007).

${ }^{17}$ H. L. Glass, Proc. IEEE 76, 151 (1988).

${ }^{18}$ Y. Chen, T. Sakai, T. Chen, S. D. Yoon, C. Vittoria, and V. G. Harris, J. Appl. Phys. 100, 043907 (2006)

${ }^{19}$ S. D. Yoon and C. Vittoria, J. Appl. Phys. 93, 8597 (2006).

${ }^{20}$ F. Licci, G. Turilli, and T. Besagni, IEEE Trans. Magn. 24, 593 (1988).

${ }^{21}$ J. F. Wang, C. B. Ponton and I. R. Harris, J. Magn. Magn. Mater. 242-245, 1464 (2002).

${ }^{22}$ R. C. Pullar and A. K. Bhattacharya, J. Mater.Sci. 36, 4805 (2001).

${ }^{23}$ M. Obol, X. Zuo, and C. Vittoria, J. Appl. Phys. 91, 7616 (2006).

${ }^{24}$ Z. W. Li, L. Chen, and C. K. Ong, J. Appl. Phys. 94, 5918 (2003). 
Özgür et al., submitted to Journal of Materials Science: Materials in Electronics, 2009 Microwave Ferrites, Part 1: Fundamental properties

${ }^{25}$ K. N. Rozanov, Z. W. Li, L. F. Chen, and M. Y. Koledintseva, J. Appl. Phys. 97, 013905 (2005).

${ }^{26}$ X. Zuo, A. Yang, S.-D. Yoon, J. A. Christodoulides, V. G. Harris, and C. Vittoria, Appl. Phys. Lett. 87, 152505 (2005).

${ }^{27}$ K. P. Chae, J. G. Lee, W. K. Kim, and Y. B. Lee, J. Magn. Magn. Mater. 248, 236 (2002).

${ }^{28}$ J.-G. Lee, J. Y. Park, Y.-J. Oh, and C. S. Kim, J. Appl. Phys. 84, 2801 (1998).

${ }^{29}$ L. Néel, J. Phys. et Radium 9, 184 (1948).

${ }^{30}$ L. Néel, Ann. Phys. 3, 137 (1948).

${ }^{31}$ R. F. Soohoo, “Theory and Application of Ferrites”, Prentice-Hall, 1960.

${ }^{32}$ B. Lax and K. J. Button, "Microwave Ferrites and Ferrimagnetics", McGraw-Hill, New York, 1962.

${ }^{33}$ S. V. Vonsovskii, Ed., "Ferromagnetic Resonance”, Pergamon Press Ltd., 1966.

${ }^{34}$ A. G Gurevich and G. A Melkov, "Magnetization Oscillations and Waves", CRC Press Inc., 1996.

${ }^{35}$ D. Polder, Phil. Mag. 40, 99 (1949).

${ }^{36}$ J. B. Goodenough, IEEE Trans. Mag. 38, 3398 (2002).

${ }^{37}$ N. Bloembergen, Phys. Rev. 572 (1950); N. Boembergen, Proc. IRE 44, 1259 (1956).

${ }^{38}$ L. Landau and E. Lifshitz, Phyzik Z. Sowjetunion 8, 153 (1935).

39 T. L. Gilbert, IEEE Trans. Magn. 40, 3443 (2004).

${ }^{40}$ C. Kittel, Phys. Rev. 73, 155 (1953).

${ }^{41}$ H. How, P. Shi, C. Vittoria, L. C. Kempel, and K. D. Trott, J. Appl. Phys. 87, 4966 (2000).

${ }^{42}$ P. C. Dorsey, P. Lubitz, D. B. Chrisey, and J. S. Horwitz, J. Appl. Phys. 79, 6338 (1996).

${ }^{43}$ E. Schlömann, Proc. Conf. on Mag. and Mag. Materials, AIEE Spec. Publ. T-91, 600 (1957).

${ }^{44}$ L. Landau and E. Lifshitz, "Electrodynamics of Continuous Media", 2nd edition, Pergamon Press, Oxford, 1984.

${ }^{45}$ E. F. Schloemann, IEEE. Trans. Magn. 34, 3830 (1998).

${ }^{46}$ J. R. Truedson, P, Kabos, K. D. McKinstry, and C. E. Patton, J. Appl. Phys. 76, 432 (1994).

${ }^{47}$ M. Sparks, R. Loudon, and C. Kittel, Phys. Rev. 122, 791 (1961).

${ }^{48}$ M. Sparks, "Ferromagnetic Relaxation Theory", New York: McGraw- Hill, 1964. 
${ }^{49}$ A. V. Nazarov, D. Ménard, J. J. Green, C. E. Patton, G. M. Argentina, and H. J. Van Hook, J. Appl. Phys. 94, 7227 (2003).

${ }^{50}$ C. E. Patton, Phys. Rev. 179, 352 (1969).

${ }^{51}$ P. E. Seiden and J. G. Grunberg, J. Appl. Phys. 34, 1696 (1963).

${ }^{52}$ N. Mo, Y.-Y. Song, and C. E. Patton, J. Appl. Phys. 97, 093901 (2005).

${ }^{53}$ N. Mo, J. J. Green, P. Krivosik, and C. E. Patton, J. Appl. Phys. 101, 023914 (2007).

${ }^{54}$ Y.-Y. Song, S. Kalarickal, and C. E. Patton, J. Appl. Phys. 94, 5103 (2003).

${ }^{55}$ Y.-Y. Song, M. S. Grinolds, P. Krivosik, and C. E. Patton, J. Appl. Phys. 97, 103516 (2005).

${ }^{56}$ S. G. Wang, S. D. Yoon, and C. Vittoria, J. Appl. Phys. 92, 6728 (2002).

${ }^{57}$ R. Karim, K. D. McKinstry, J. R. Truedson, and C. E. Patton, IEEE Trans Magn. 28, 3225 (1992).

${ }^{58}$ J. Smit and H. P. J. Wijn, "Ferrites: Physical Properties of Ferrimagnetic Oxides in Relation to Their Technical Applications", Philips Technical Library, Eindhoven, Netherlands, 1959.

${ }^{59}$ W. H. von Aulock (Ed.), A. S. Boxer, J. F. Ollom, and R. F. Rauchmiller, "Handbook of Microwave Ferrite Materials", Academic Press, London, 1965.

${ }^{60}$ R. Valenzuela, “Magnetic Ceramics”, Cambridge University Press, 1994.

${ }^{61}$ A. Goldman, "Modern Ferrite Technology”, 2nd Ed., Springer-Verlag, 2006.

${ }^{62}$ A. Ataie, I. R. Harris, and C. B. Ponton, J. Mat. Sci. 30, 1429 (1995).

${ }^{63}$ A. J. Kerecman, T. R. AuCoin, and W. P. Dattilo, J. Appl Phys. 40, 1416 (1969).

${ }^{64}$ D. Lisjak and M. Drofenik, J. Appl. Phys. 93, 8011 (2003).

${ }^{65}$ G. Xiong and Z. H. Mai, J. Appl. Phys. 88, 519 (2000).

${ }^{66}$ S. G. Lee and S. J. Kwon, J. Mag. Magn. Mat. 153, 279 (1996).

${ }^{67}$ K. T. Han, Phys. Stat. Sol. (a) 155, 215 (1996).

${ }^{68}$ J. P. Chen, C. M. Sorensen, K. J. Klabunde, G. C. Hadjipanayis, E. Devlin, and A. Kostikas, Phys. Rev. B. 54, 9288 (1996).

${ }^{69}$ I. Wane, A. Bessaudou, F. Cosset, A. Celerier, C. Girault, J. L. Decossas, and J. Vareille, J. Magn. Magn. Mater. 211, 309 (2000).

${ }^{70}$ B. S. Kwak, K. Zhang, E. P. Boyd, A. Erbif, and B. J. Wilkens, J. Appl. Phys. 69, 767 (1991).

${ }^{71}$ S. Pignard, H. Vincent, and J. P. Senateur, Thin Solid Films 350, 119 (1999).

${ }^{72}$ M. Matsuoka, Y. Hoshi, M. Naoe, and S. Yamanaka, IEEE Trans. Magn. 20, 800 (1984). 
Özgür et al., submitted to Journal of Materials Science: Materials in Electronics, 2009 Microwave Ferrites, Part 1: Fundamental properties

${ }^{73}$ E. Suzuki, Y. Hoshi, and M. Naoe, J. Appl. Phys. 83, 6250 (1998).

${ }^{74}$ A. Morisako, X. Liu, M. Matsumoto, and M. Naoe, J. Appl. Phys. 81, 4374 (1997).

${ }^{75}$ Q. Fang, H. Bao, D. Fang, and J. Wang, J. Appl. Phys. 95, 6360 (2004).

${ }^{76}$ S. Y. An, S. W. Lee, I.-B. Shim, and C. S. Kim, IEEE Trans. Magn. 37, 2585 (2001).

${ }^{77}$ H. Dötsch, D. Mateika, P. Röschmann, and W. Tolksdorf, Mater. Res. Bull. 18, 1209 (1983).

${ }^{78}$ S. Capraro, J. P. Chatelon, H. Joisten, M. Le Berre, B. Bayard, D. Barbier, and J. J. Rousseau, J. Appl. Phys., 93, 9898 (2003).

${ }^{79}$ S. H. Gee, Y.K. Hong, D.W. Erickson, T. Tanaka, and M.H. Park, J. Appl. Phys. 93, 7507 (2003).

${ }^{80}$ T. L. Hylton, M. A. Parker, and J. K. Howard, Appl. Phys. Lett. 61, 867 (1992).

${ }^{81}$ S. R. Shinde, R. Ramesh, S. E. Lofland, S. M. Bhagat, S. B. Ogale, R. P. Sharma, and T. Venkatesan, Appl. Phys. Lett. 72, 3443 (1998).

${ }^{82}$ P. C. Dorsey, D. B. Chrisey, J. S. Horwitz, P. Lubitz, and R. C. Y. Auyeung, IEEE Trans. Magn. 30, 4512 (1994).

${ }^{83}$ S. A. Oliver, S. D. Yoon, I. Kouzulin, M. L. Chen, and C. Vittoria, Appl. Phys. Lett. 76, 3612 (2000).

${ }^{84}$ P. R. Ohodnicki, K. Y. Goh, Y. Hanlumyuang, K. Ramos, M. E. McHenry, Z. Cai, K. Ziemer, H. Morkoç, N. Biyikli, Z. Chen, C. Vittoria, and V. G. Harris, Appl. Phys. 101, 09M521 (2007).

${ }^{85}$ E. Lacroix, P. Gerard, G. Marest, and M. Dupuy, J. Appl. Phys. 69, 4770 (1991).

${ }^{86}$ A. G. Smolenski and A. A. Andreev, Bull. Acad. Sci. 25, 1405 (1961).

${ }^{87}$ G. Asti, F. Bolzoni, J. M. Le Breton, M. Ghidini, A. Morel, M. Solzi, F. Kools and P. Tenaud, J. Magn. Magn. Mater. 272, E1845 (2004).

${ }^{88}$ G. Wiesinger, M. Müller, R. Grössinger, M. Pieper, A. Morel, F. Kools, P. Tenaud, J.M. Le Breton, J. Kreisel, "Substituted Ferrites Studied by Nuclear Methods", Phys. Status Sol. (a) 189, 499 (2002).

${ }^{89}$ M. W. Pieper, F. Kools and A. Morel, Phys. Rev. B 65, 184397 (2002).

${ }^{90}$ H.S. Cho and S.S. Kim. IEEE Trans. Magn. 35, 3151 (1999).

${ }^{91}$ J. Kreisel, H. Vincent, F. Tasset, M. Paste, and J.P. Ganne, J. Magn. Magn. Mater. 224, 17 (2001).

92 J. Kreisel, H. Vincent, F. Tasset, M. Paste and P. Wolfers, Physica B 276-278, 688 (2000).

${ }^{93}$ H. J. Zhang, X. Yao and L. Y. Zhang, J. Magn. Magn. Mater. 241, 441 (2002).

${ }^{94}$ P. Wartewig, M.K. Krause, P. Esquinazi, S. Rosler and R. Sonntag, J. Magn. Magn. Mater. 192, 83 (1999). 
Özgür et al., submitted to Journal of Materials Science: Materials in Electronics, 2009 Microwave Ferrites, Part 1: Fundamental properties

${ }^{95}$ Q.Y. Feng and L. Jen, IEEE Trans. Magn. 38, 1391 (2002).

${ }^{96}$ J. Kreisel, H. Vincent, F. Tasset and P. Wolfers, J. Magn. Magn. Mater. 213, 262 (2000).

${ }^{97}$ G. Litsardakis, I. Manolakis and K. Efthimiadis, J. Alloys and Compounds 427, 194 (2007).

${ }^{98}$ M. V. Rane, D. Bahadur, A. K. Nigan and C. M. Srivastava, J. Magn. Magn. Mater. 192, 288 (1999).

${ }^{99}$ G. K. Thompson and B. J. Evans, J. Appl. Phys. 75, 6643 (1994).

${ }^{100}$ O. Kohmoto, T. Tsukada and S. Sato, Jpn. J. Appl. Phys. 29, 1944 (1990).

${ }^{101}$ Y. Kawai, V.A.M. Brabers, Z. Simsa and J. H. J. Dalderop, J. Magn. Magn. Mater. 196-197, 309 (1999).

${ }^{102}$ V. Babu and P. Padaikathan, J. Magn. Magn. Mater. 241, 85 (2002).

${ }^{103}$ P. Shi, H. How, X. Zuo, S. D. Yoon, S. A. Oliver, and C. Vittoria, IEEE Trans. Magn. 37, 2389 (2001).

${ }^{104}$ M. Pal, P. Brahma, B. R. Chakraborty, and D. Chakravorty, Jpn. J. Appl. Phys. 36, 2163 (1997).

${ }^{105}$ P. Brahma, S. Banerjee, S. Chakraborty, and D. Chakravorty, J. Appl. Phys. 88, 6526 (2000).

${ }^{106}$ P. A. Mariño-Castellanos, J. Anglada-Rivera, , A. Cruz-Fuentes and R. Lora-Serrano, J. Magn. Magn. Mat. 280, 214 (2004).

${ }^{107}$ M. Küpferling, R. Grössinger, M. W. Pieper, G. Wiesinger, H. Michor, C. Ritter, and F. Kubel, Phys. Rev. B 73, 144408 (2006).

${ }^{108}$ J. F. Wang, C. B. Ponton, R. Grössinger, and I. R. Harris, J. Alloys and Compounds 369, 170 (2004).

109 J. F. Wang, C. B. Ponton, and I. R. Harris, IEEE Trans. Magn. 38, 2928 (2002).

${ }^{110}$ F. K. Lotgering, J. Phys. Chem. Solids 35, 1633 (1974).

${ }^{111}$ V.L. Moruzzi and M.W. Shafer, J. Am. Ceram. Soc. 43, 367 (1960).

112 C. M. Fang, F. Kools, R. Metselaar, G. deWith, and R. A. de Groot, J. Phys. Condensed Matter 15, 6229 (2003).

113 G. Albanese, M. Carbucicchio, and G. Asti, Appl. Phys. 11, 81 (1976).

114 R. A. Braden, I. Cordon, and R. L. Harvey, IEEE Trans. Magn. 2, 43 (1966).

${ }^{115}$ D. Autissier, A. Podembski, C. Jacquiod, J. de Physique IV 7, 409 (1997).

${ }^{116}$ M. Matsumoto and Y. Miyata, J. Appl. Phys. 79, 5486 (1996).

${ }^{117}$ E. Pollert, Prog. Crystal Growth and Charact. 11, 155 (1985).

${ }^{118}$ B. X. Gu, J. Appl. Phys. 75, 4114 (1994). 
Özgür et al., submitted to Journal of Materials Science: Materials in Electronics, 2009 Microwave Ferrites, Part 1: Fundamental properties

${ }^{119}$ B. X. Gu, J. Appl. Phys. 70, 372 (1991).

${ }^{120}$ B. X. Gu, J. Appl. Phys. 71, 5103 (1992).

${ }^{121}$ F. Leccabue, R. Panizzieri, S. Garcia, N. Suarez, J.L. Sanchez, O. Ares, and Rong Hua Xue, J. Mater. Sci. 25, 2765 (1990).

${ }^{122}$ M. El-Saadawy, Inter. Cer. Rev. 52, 206 (2003).

${ }^{123}$ B. X. Gu, H. X. Lu and Y. W. Du, J. Magn. Magn. Mater 31-34, 803 (1983).

${ }^{124}$ Darja Lisjak, Darko Makovec, and Miha Drofenik, J. Mater. Res. 21, 420 (2006).

${ }^{125}$ P. B. Braun, Philips Res. Rep. 12, 491 (1957).

${ }^{126}$ H. J. Zhang, X. Jia, X. Yao, and L. Y. Zhang, Rare Metals 23, 27 (2004).

${ }^{127}$ D. Lisjak and M. Drofenik, J. Magn. Magn. Mater. 272-276, E1817 (2004).

${ }^{128}$ M. N. Afsar, D. Lisjak, A. Bahadoor, and Y. Wang, IEEE Trans. Magn. 41, 3472 (2005).

${ }^{129}$ H. Zhang, Z. Liu, X. Yao, L. Zhang, and M. Wu, Mater. Sci. Eng. B 97, 160 (2003).

${ }^{130}$ D. Lisjak, V. B. Bregar, and M. Drofenik, J. Magn. Magn. Mater. 310, 2558 (2007).

131 T. Nakamura and K.-I. Hatakeyama, IEEE Trans. Magn. 36, 3415 (2000).

${ }^{132}$ H. J. Kwon, J. Y. Shin, and J. Y. Oh, J. Appl. Phys. 75, 6109 (1994).

${ }^{133}$ M. Obol and C. Vittoria, J. of Appl. Phys. 94, 4013 (2003).

${ }^{134}$ M. Obol, and C. Vittoria, IEEE Trans. Magn. 39, 3103 (2003).

${ }^{135}$ M. Obol, and C. Vittoria, J. Magn. Magn. Mater. 272-276, E1799 (2004).

${ }^{136}$ M. Obol, and C. Vittoria, J. Magn. Magn. Mater. 265, 290 (2003).

${ }^{137}$ J. Temuujin, M. Aoyama, M. Senna, T. Masuko, C. Ando, and H. Kishi, J. Solid State Chem. 177, 3903 (2004).

${ }^{138}$ X. Li, R. Gong, Z. Feng, J. Yan, X. Shen, and H. He, J. Amer. Cer. Soc. 89, 1450 (2006).

${ }^{139}$ R. Muller, J. Magn. Magn. Mater. 101, 230 (1991).

${ }^{140}$ R. Muller, J. Magn. Magn. Mater. 120, 61 (1993).

${ }^{141}$ F. Haberey, IEEE Trans. Magn. 23, 29 (1987).

${ }^{142}$ H. Zhang, Z. Liu, X. Yao, L. Zhang, and M. Wu, J. Sol-Gel Sci. Technol. 27, 277 (2003).

${ }^{143}$ X. Wang, D. Li, L. Lu, and X. Wang, J. Alloys and Compounds 237, 45 (1996).

${ }^{144}$ H. Graetsch, F.Harberey, R. Leckebusch, M. S. Rosenberg, and K. Sahl, IEEE Trans. Magn. 20, 495 (1984).

${ }^{145}$ H. Kojima, C. Miyakawa, T. Sato, and K. Goto, Jpn. J. Appl. Phys. 24, 51 (1985).

${ }^{146}$ F. Haberey and P. Wiesemann, IEEE Trans. Magn. 24, 2112 (1988). 
Özgür et al., submitted to Journal of Materials Science: Materials in Electronics, 2009 Microwave Ferrites, Part 1: Fundamental properties

${ }^{147}$ A. Paoluzi, G. Turilli, F. Licci, and S. Rinaldi, J. Appl. Phys. 61, 3301 (1987).

${ }^{148}$ S. Rinaldi, F. Licci, A. Paoluzi, and G. Turilli, J. Appl. Phys. 60, 3680 (1986).

${ }^{149}$ K. K. Mallick, P. Shepherd, and R. J. Green, J. Magn. Magn. Mater. 312, 418 (2007).

${ }^{150}$ A. M. Abo El Ata, F. M. Reicha, and M. M. Ali, J. Magn. Magn. Mater. 292, 17 (2005).

${ }^{151}$ D. El Kony and S. A. Saafan, J. Magn. Magn. Mater. 267, 46 (2003).

152 S. Ruan, B. Xu, H. Suo, F. Wu, S. Xiang, and M. Zhao, J. Magn. Magn. Mater. 212, 175 (2000).

${ }^{153}$ P. Shepherd, K. K. Mallick, and R. J. Green, J. Mater. Sci.: Materials in Electronics 18, 527 (2007).

${ }^{154}$ D. El-Kony, S. A. Saafan, A. M. Abo El Ata, Egyptian Journal of Solids 23, 137 (2000).

155 A. M. Abo El Ata, M. K. El Nimr, D. El Kony, and A.H. Al-Hammadi, J. Magn. Magn. Mater. 204, 36 (1999).

${ }^{156}$ S. P. Kuntsevich, and V. P. Palekhin, Soviet Physics - Solid State 15, 2314 (1974).

${ }^{157}$ Y. Maeda, S. Sugimoto, D. Book, H. Ota, M.; Kimura, H. Nakamura, T. Kagotani, and M. Homma, Materials Transactions JIM 41, 567 (2000).

${ }^{158}$ F. K. Lotgering, U. Enz, and J. Smit, Philips Res. Rep. 16, 441 (1961).

${ }^{159}$ A. Paoluzi, F. Licci, O. Moze, G. Turilli, A. Deriu, G. Albanese, and E. Calabrese, J. Appl. Phys. 63, 5074 (1988).

${ }^{160}$ Y. Takada, T. Nakagawa, M. Tokunaga, Y. Fukuta, T. Tanaka, T. A. Yamamoto, T. Tachibana, S. Kawano, Y. Ishii, and N. Igawa, J. Appl. Phys. 100, 043904 (2006).

${ }^{161}$ P. M. G. Nambissan, C. Upadhyay, and H. C. Verma, J. Appl. Phys. 93, 6320 (2003).

${ }^{162}$ T. Sato, K. Haneda, M. Seki, and T. Iijima, Appl. Phys. A: Solids Surf. 50, 13 (1990).

${ }^{163}$ T. Kamiyama, K. Haneda, T. Sato, S. Ikeda, and H. Asano, Solid State Commun. 81, 563 (1992).

${ }^{164}$ H. H. Hamdeh, J. C. Ho, S. A. Oliver, R. J. Willey, G. Olivery, and G. Busca, J. Appl. Phys. 81, 1851 (1997).

165 B. Jeyadevan, K. Tohji, and K. Nakatsuka, J. Appl. Phys. 76, 6325 (1994).

${ }^{166}$ W. Schiessl, W. Potzel, H. Karzel, M. Steiner, and G. M. Kalvius, Phys. Rev. B 53, 9143 (1996).

${ }^{167}$ J. C. Ho, H. H. Hamdeh, Y. Y. Chen, S. H. Lin, Y. D. Yao, R. J. Willey, and S. A. Oliver, Phys. Rev. B 52, 10122 (1995).

${ }^{168}$ M. Tachiki, Prog. Theor. Phys. 23, 1055 (1960).

${ }^{169}$ S. D. Bhame and P. A. Joy, J. Appl. Phys. 99, 073901 (2006). 
${ }^{170}$ Y. Suzuki, R. B. van Dover, E. M. Gyorgy, Julia M. Phillips, V. Korenivski, D. J. Werder, C. H. Chen, R. J. Cava, J. J. Krajewski, and W. F. Peck, Jr., K. B. Do, Appl. Phy. Lett. 68, 714 (1996).

${ }^{171}$ G. Hu, J. H. Choi, C. B. Eom, V. G. Harris, and Y. Suzuki, Phys. Rev. B 62, R779 (2000).

${ }^{172}$ J. Dash, R. Krishnan, N. Venkataramani, S. Prasad, S. N. Shringi, P. Kishan, and N. Kumar, J. Magn. Magn. Mater. 152, L1 (1996).

${ }^{173}$ S. Capdeville, P. Alphonse, C. Bonningue, L. Presmanes, and P. Tailhades, J. Appl. Phys. 96, 6142 (2004).

${ }^{174}$ S. A. Chambers, R. F. C. Farrow, S. Maat, M. F. Toney, L. Folks, J. G. Catalanoc, T. P. Trainorc and G. E. Brown, J. Magn. Magn. Mater. 246, 124 (2002).

${ }^{175}$ F.-X. Cheng, J.-T. Jia, Z.-G. Xu, B. Zhou, C.-S. Liao, C.-H. Yan, L.-Y. Chen, and H.B. Zhao, J. Appl. Phys. 86, 2727 (1999).

${ }^{176}$ Y. Suzuki, Annu. Rev. Mater. Res. 31, 265 (2001).

${ }^{177}$ R. W. McCallum, K. W. Dennis, D. C. Jiles, J. E. Snyder, and Y. H. Chen, Low Temp. Phys. 27, 266 (2001).

${ }^{178}$ I. M. L. Billas, A. Chatelain, and W.A. de Heer, Science 265, 1682 (1994).

179 J. Shi, S. Gider, K. Babcock and D.D. Awschalom, Science 271, 937 (1996).

${ }^{180}$ M. Kishimoto, Y. Sakurai, and T. Ajima, J. Appl. Phys. 76, 7506 (1994).

${ }^{181}$ Y. I. Kim, H. Kang, D. Kim, and C. S. Lee, Bull. Korean Chem. Soc. 24, 593 (2003).

${ }^{182}$ M. George, A. M. John, S. S. Nair, P.A. Joy, and M. R. Anantharaman, J. Magn. Magn. Mater. 302, 190 (2006).

${ }^{183}$ E. S. Murdock, R. F. Simmons, and R. Davidson, IEEE Trans. Magn. 28, 3078 (1992).

${ }^{184}$ B. P. Rao, C.-O. Kim, C. Kim, I. Dumitru, L. Spinu, and O. F. Caltun, IEEE Trans. Magn. 42, 2858 (2006).

${ }^{185}$ A. S. Albuquerque, J. D. Ardisson, W. A. A. Macedo, and M. C. M. Alves, J. Appl. Phys. 87, 4352 (2000).

${ }^{186}$ D. H. Chen and Y. Y. Chen, J. Colloid Interface Sci. 235, 9 (2001).

${ }^{187}$ S. Z. Zhang and G. L. Messing, J. Am. Ceram. Soc. 73, 61 (1990).

${ }^{188}$ C. S. Kim, Y. S. Yi, K.-T. Park, H. Namgung, and J.-G. Lee, J. Appl. Phys. 85, 5223 (1999).

${ }^{189}$ Z. Yue, J. Zhou, L. Li, H. Zhang, and Z. Gui, J. Magn. Magn. Mater. 208, 55 (2000).

${ }^{190}$ S. Komarneni, M. C. D’Arrigo, C. Leonelli, G. C. Pellacani and H. Katsuki, J. Am. Ceram. Soc. 81, 3041 (1998).

${ }^{191}$ C. Rath, N. C. Mishra, S. Anand, R. P. Das, K. K. Sahu, C. Upadhyay, and H. C. Verma, Appl. Phys. Lett. 76, 475 (2000). 
Özgür et al., submitted to Journal of Materials Science: Materials in Electronics, 2009 Microwave Ferrites, Part 1: Fundamental properties

${ }^{192}$ A. Singh, A. Verma, O. Thakur, C. Prakash, T. Goel, and R. G. Mendiratta, Mater. Lett. 57, 1040 (2003).

${ }^{193}$ Z. Cheng, H. Yang, L. Yu, Y. Cui, and S. Feng, J. Magn. Magn. Mater. 302, 259 (2006).

${ }^{194}$ X. Batlle, X. Obradors, M. Medarde, J.R. Carvajal and M. Pernet, J. Magn. Magn. Mater. 124, 228 (1993).

195 S. Maensiria, C. Masingboona, B. Boonchomb, and S. Seraphinc, Scripta Materialia 56, 797 (2007).

${ }^{196}$ F. Bertaut and F. Forrat, Compt. Rend. Acad. Sci. Paris 242, 382 (1956).

${ }^{197}$ G. P. Rodrigue, Proc. IEEE 76, 121 (1988).

${ }^{198}$ M. Pardavi-Horvath, J. Magn. Magn. Mater. 215-216, 171 (2000).

${ }^{199}$ C. Vittoria, P. Lubitz, P. Hansen, and W. Tolksdorf, J. Appl. Phys. 57, 3699 (1985).

${ }^{200}$ T. Okuda, N. Koshizuka, K. Hayashi, T. Takahashi, H. Kotani, and H. Yamamoto, IEEE Trans. Magn. 23, 3491 (1987).

${ }^{201}$ S.-Y. Sung, X. Qi, and B. J. H. Stadler, Appl. Phys. Lett. 87, 121111 (2005)

${ }^{202}$ M. Gomi, T. Tanida, and M. Abe, J. Appl. Phys. 57, 3888 (1985).

203 J.-P. Krumme, V. Doormann, and P. Willich, J. Appl. Phys. 57, 3885 (1985).

${ }^{204}$ Y. Dumont, N. Keller, E. Popova, D. S. Schmool, S. Bhattacharya, B. Stahl, M. Tessier, and M. Guyot, J. Appl. Phys. 97, 10 G108 (2005).

${ }^{205}$ S. Kahl and A. M. Grishin, J. Appl. Phys. 93, 6945 (2003).

${ }^{206}$ H. Kidoh, A. Morimoto, and T. Shimizu, Appl. Phys. Lett. 59, 237 (1991).

${ }^{207}$ Y. H. Kim, J. S. Kim, S. I. Kim, and M. Levy, J. Korean Phys. Soc. 43, 400 (2003).

${ }^{208}$ P. C. Dorsey, S. E. Bushnell, R. G. Seed, and C. Vittoria, J. Appl. Phys. 74, 1242 (1993) .

${ }^{209}$ T. Okuda, T. Katayama, H. Kobayashi, N. Kobayashi, K. Satoh and H. Yamamoto, J. Appl. Phys. 67, 4944 (1990).

${ }^{210}$ P. Hansen and J. P. Krumme, Thin Solid Films 114, 69 (1984). 